\title{
TROPHIC INTERACTIONS AND ECOSYSTEM MANAGEMENT AT THE NEW ZEALAND SUBANTARCTIC ISLANDS
}

BY

\section{SEAN DAVID COOPER}

\author{
A thesis \\ submitted to Victoria University of Wellington \\ in fulfilment of the requirements for the degree of \\ Master of Science
}

Victoria University of Wellington

2014 


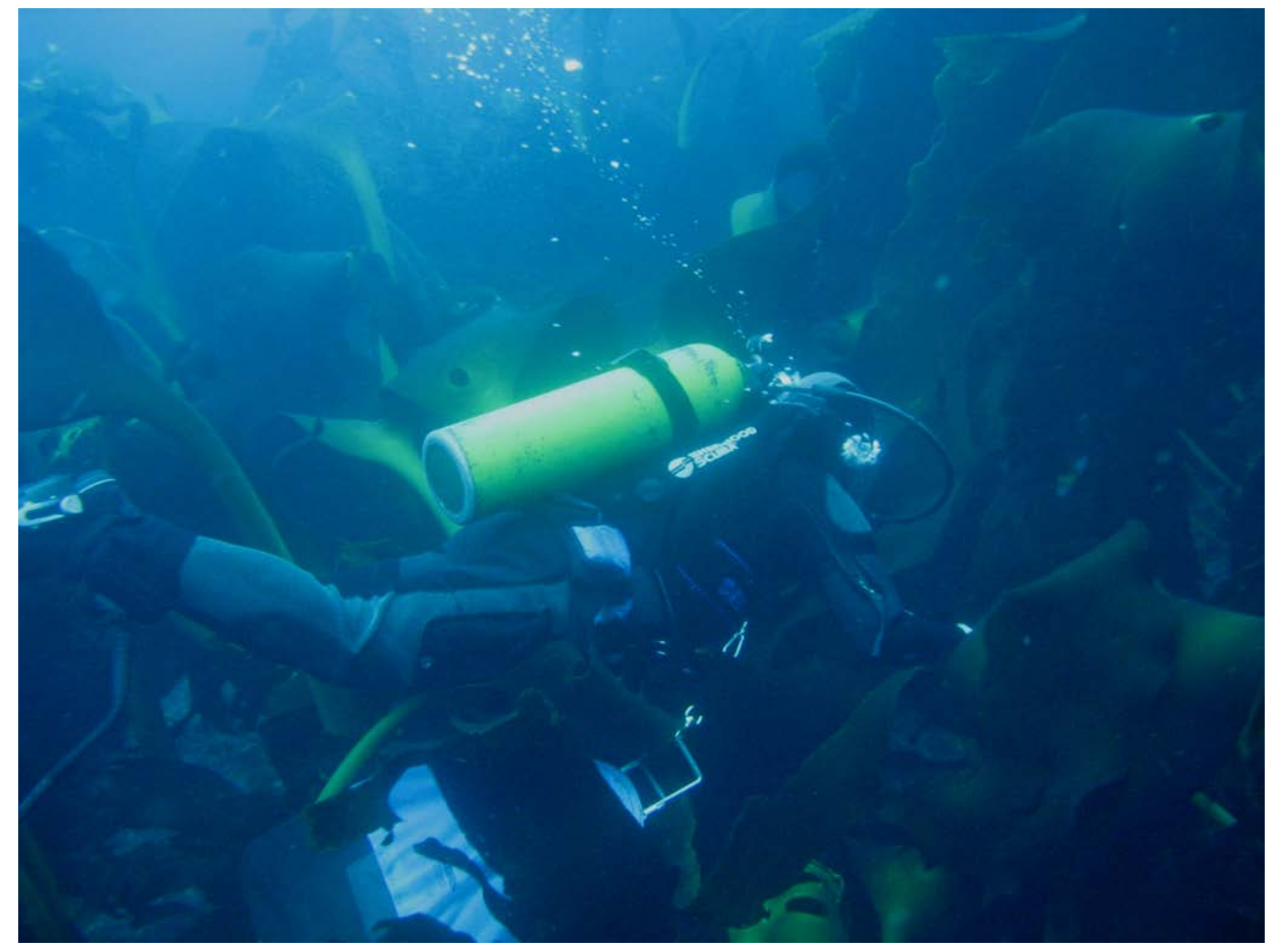

FRONTISPIECE: Antipodes Island bull kelp, Durvillaea sp. Antipodes Island. 


\begin{abstract}
Ecosystem-based management (EBM) has become an increasingly popular concept for government agencies to incorporate into management planning strategies. The basic idea behind EBM is that an ecosystem remains intact, resilient and productive in the long-term, to provide for ecological, social, cultural and economic benefits. The problem that decision makers face is that there is often little information regarding the structure and functioning of ecosystems upon which to base meaningful decisions. A further complication is that governance of the environment is highly sectoral both across government and within agencies. This often leads to fractured management between the terrestrial, freshwater and marine environments, potentially risking biodiversity loss and the stability of ecosystems.

Small oceanic islands may potentially be model ecosystems for undertaking ecological studies, due to their constrained spatial extent and often unmodified condition. The New Zealand Subantarctic Islands, which are remote and largely unmodified, provide a natural laboratory to study the structure and functioning of ecosystems. I undertook stable isotope and water nutrient sampling to describe the trophic structure, trophic interactions and the drivers of the Antipodes and Bounty Islands, two of the islands in New Zealand's Subantarctic region. These islands have high conservation value and are an important area for breeding seabirds and marine mammals, but there have been no studies at these islands to understand how they function and what the connections are between the terrestrial and marine environments.
\end{abstract}

Using the stable isotope signatures of nitrogen $\left(\delta^{15} \mathrm{~N}\right)$ and carbon $\left(\delta^{13} \mathrm{C}\right)$ from a wide range of common marine and terrestrial species at both islands, I described the trophic structure of each island. I found that the islands had a similar number of trophic levels and that omnivory was present beyond secondary consumers and below top level predators. Antipodes Island had a more complex food web than the Bounty Islands, but both islands showed strong linkages between the terrestrial and 
marine environments at both a local scale and with habitats beyond the sovereignty area of New Zealand.

A basic two-source mixing model was used to determine the carbon sources that were important at each island. It was found that the Antipodes Island marine communities were influenced by phytoplankton, but that kelp was also an important contributor of carbon to consumers' diets. In contrast, at the Bounty Islands, phytoplankton was the sole carbon source in marine communities. Terrestrial species at both islands had a marine-derived carbon component to their diets, with Antipodes Island terrestrial species incorporating a combination of terrestrialderived and marine-derived carbon. The Bounty Islands' terrestrial species were completely reliant on marine-derived carbon that was linked to phytoplankton. To further test the diets of species, Isosource was used to reconstruct the diets of the most common marine invertebrates and terrestrial species, again demonstrating strong marine-terrestrial links.

To determine if there was any correlation between the distance from shore, water nutrient concentrations and phytoplankton stable isotope signatures, samples were collected in open ocean sites across the Campbell Plateau and within 12 nautical miles of each island. It was found that the nitrate levels of Antipodes Island water samples decreased with distance towards the island and that nitrate and dissolved reactive phosphorous levels increased with distance towards the Bounty Islands.

This research has clearly demonstrated that there is a strong link between the marine and terrestrial realms at both islands and at spatial scales beyond the islands. The current management of the islands requires this new information to be taken into consideration in future management planning, so that trophic connections are maintained across realms. Further work is required across government and within agencies to bring legislation, policy and science into an integrated framework across sectors. This will allow environmental managers to reduce threats at the ecosystem level to minimise biodiversity loss and the risk of degradation of ecosystems, to 
protect New Zealand's long-term biodiversity, social, cultural and economic prosperity. 


\section{Acknowledgements}

To James Bell my supervisor- thank you for your providing thoughtful guidance and keeping me focused on the bigger picture. Your knowledge has been invaluable in the writing of this thesis. Thank you for being supportive of my working situation and for providing quick and meaningful comments back in the last few weeks.

I would like to acknowledge the funding to undertake this research by the Department of Conservation (DOC) and also the time away from work to complete this study. I could not have achieved this without the generous support of my manager Hilary Aikman. Thank you to the Southern Islands DOC staff for their logistical support and the permits, and to Irene Pohl for help with mapping. This research was conducted under the following permits: Ministry of Fisheries Special Permit 412, Department of Conservation Entry Permit SO-24882-LND, Department of Conservation Research and Collection Permit SO-24881-RES. Also, thank you to the Department of Conservation Animal Ethic committee for the approval of permits to collect protected species tissue.

Henk Haazen and Jeremy Carroll from the crew of the expedition vessel Tiamathank you for providing so many wonderful memories during this voyage, your generosity and friendship will stay with me for a lifetime. Your expert sailing in challenging environments kept me safe, the best "milos" on the seven seas kept me warm and your dive tender driving got me to the most amazing dive sites I have ever been to.

Greig Funnell, Don Neale and Debbie Freeman were invaluable, reliable and trusted dive buddies and provided a great atmosphere in the field. The bird team on Antipodes Island and in particular Erica helped to find some feathers and trap the mice, thank you for the time you took to do this and the use of some very valuable cheese. 
Thank you to NIWA, particularly Sarah Bury for loaning me the water filtering equipment and the useful advice before the expedition and the advice during the writing of this thesis.

My mother provided valuable encouragement (as always) during the time it has taken to write this thesis, thank you for the encouragement and support of my family.

Last but not least, thank you to my partner for your support and patience. You inspire me to keep challenging myself and I dedicate this thesis to you. 


\section{TABLE OF CONTENTS}

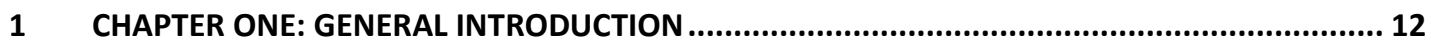

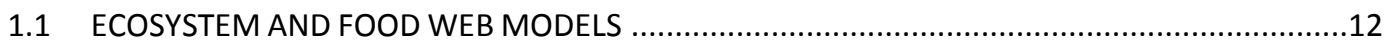

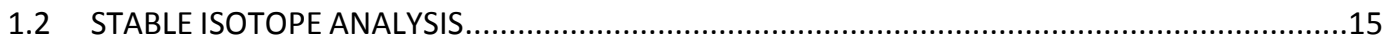

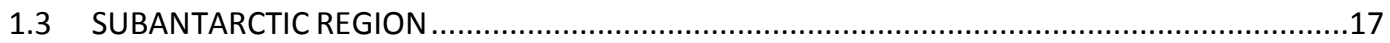

1.4 STUDY SITES

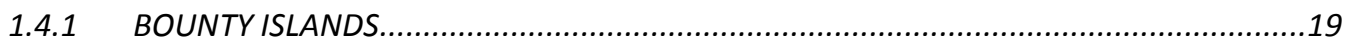

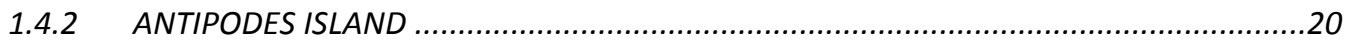

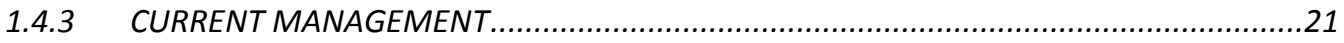

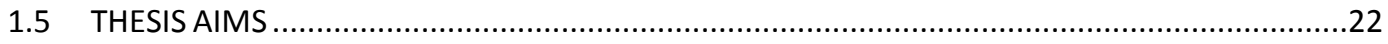

2 CHAPTER TWO: TROPHIC STRUCTURE AT THE BOUNTY AND ANTIPODES ISLANDS ............... 26

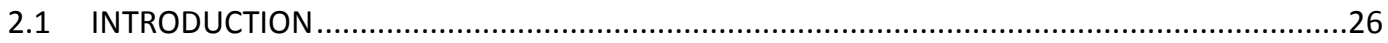

2.2 METHODS

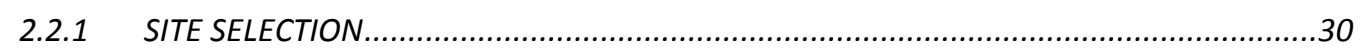

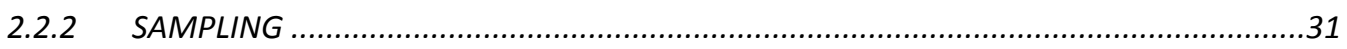

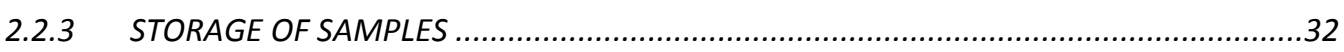

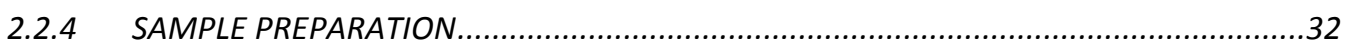

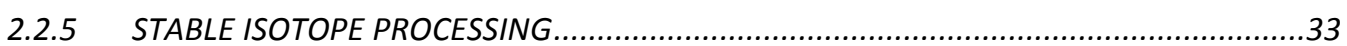

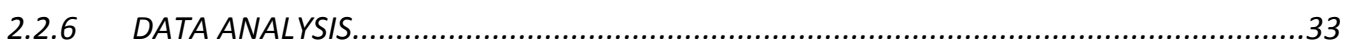

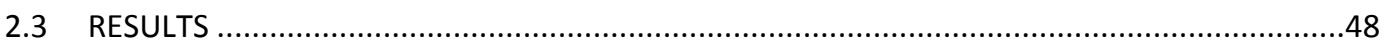

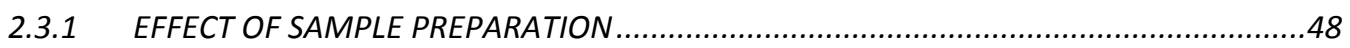

2.3.2 ISOTOPIC COMPOSITION OF ANTIPODES ISLAND AND BOUNTY ISLANDS ..................48

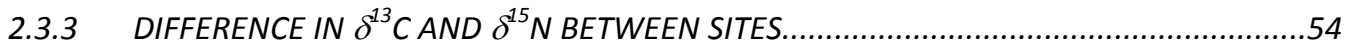

2.3.4 DIFFERENCE IN $\delta^{13} C$ AND $\delta^{15} N$ BETWEEN THE BOUNTY AND ANTIPODES ISLANDS.....55

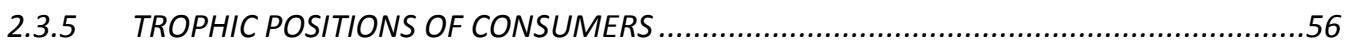

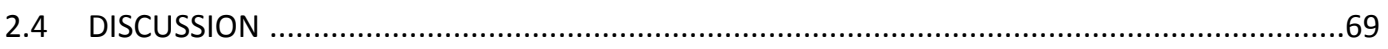

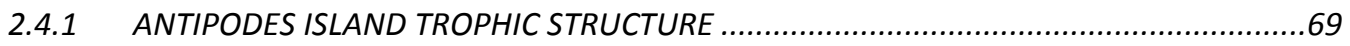

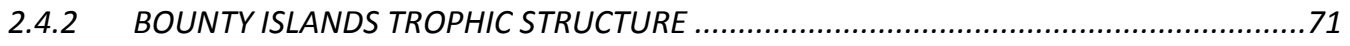

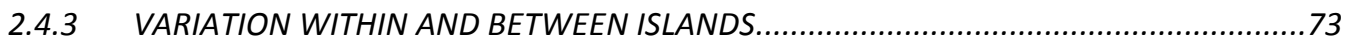

2.4.4 LIMITATIONS AND BENEFITS OF THIS STUDY …................................................... 74

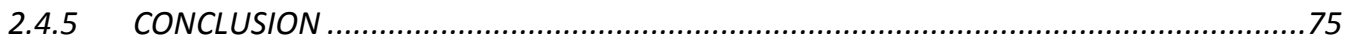

3 CHAPTER THREE: FOOD WEB DRIVERS AT ANTIPODES ISLAND AND THE BOUNTY ISLANDS 76

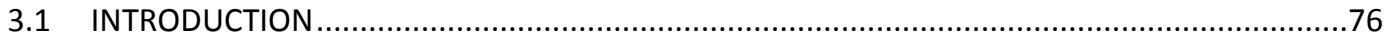

3.2 MATERIALS AND METHODS

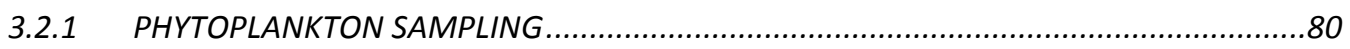

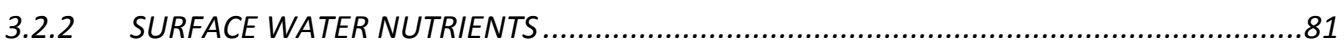

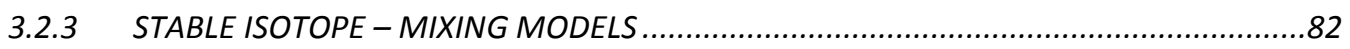

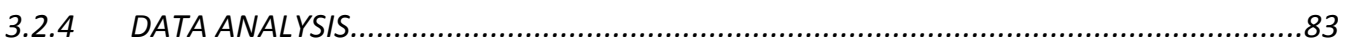

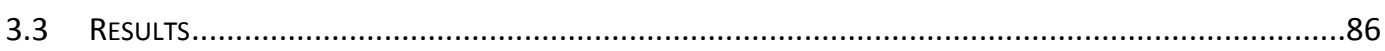

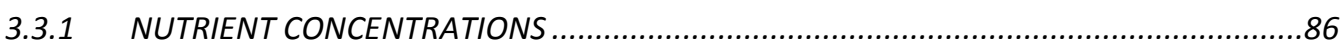

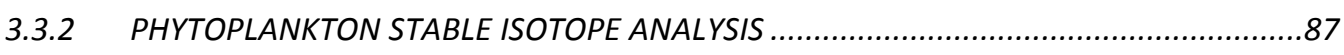

3.3.3 WATER NUTRIENT AND PHYTOPLANKTON TRANSECTS .............................................8

3.3.4 CONTRIBUTION OF KELP, PHYTOPLANKTON AND TERRESTRIAL CARBON TO

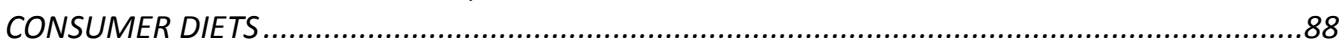

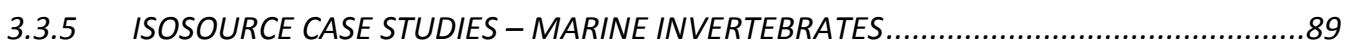

3.3.6 ISOSOURCE CASE STUDIES: MARINE-TERRESTRIAL LINKAGES................................91

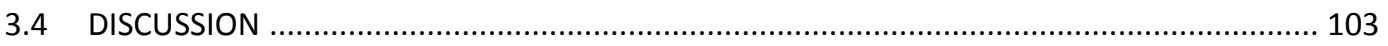




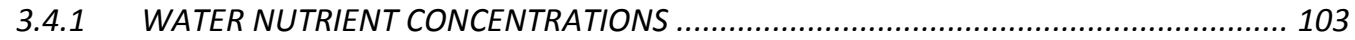

3.4.2 WATER NUTRIENT AND STABLE ISOTOPE TRANSECTS .......................................... 104

3.4.3 CONTRIBUTION OF KELP, PHYTOPLANKTON AND TERRESTRIAL CARBON TO

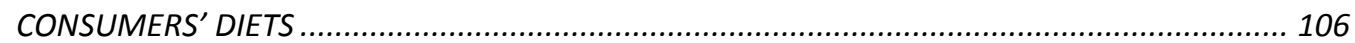

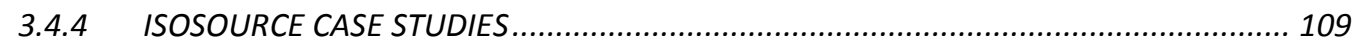

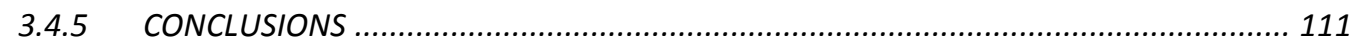

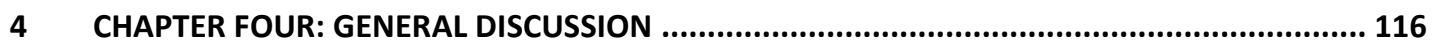

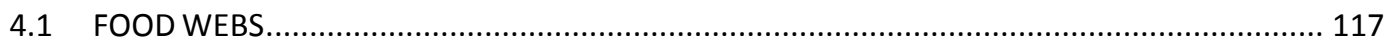

4.2 IMPLICATIONS FOR ENVIRONMENTAL PROTECTION AND MANAGEMENT ......................... 122

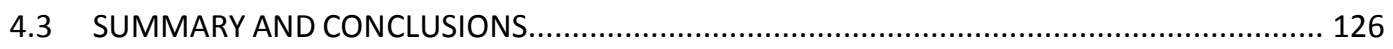

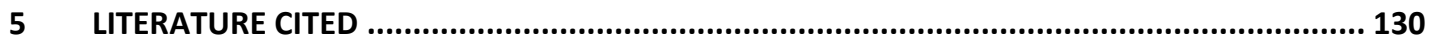




\section{List of Figures}

FIGURE 1-1 MAP SHOWING LOCATION OF THE BOUNTY ISLANDS AND ANTIPODES ISLAND.

FiguRE 1-2 DEPOT ISLAND, THE BOUNTY ISLANDS, DEMONSTRATING THE ABUNDANCE OF SEABIRDS (ERECT-CRESTED PENGUINS AND SALVIN'S ALBATROSS) ON THE ISLANDS.

FIGURE 1-3 ANTIPODES ISLAND, WITH PENGUIN COLONY ON THE SLOPE LEADING UP TO MOUNT WATERHOUSE.........25

FIGURE 2-1 MAP SHOWING THE LOCATION OF THE BOUNTY AND ANTIPODES ISLANDS.......................................45

FIGURE 2-2 MAPS SHOWING THE SITES WHERE SAMPLES WERE COLLECTED FOR STABLE ISOTOPE ANALYSIS AT THE

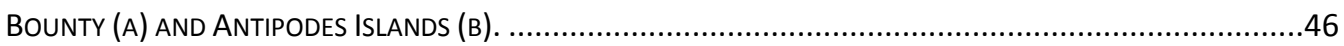

FIGURE 2-3 PHOTOGRAPH OF WATER-FILTERING APPARATUS TO COLLECT PHYTOPLANKTON..................................47

FIGURE 2-4 PLOT OF CARBON AND NITROGEN STABLE ISOTOPE RATIOS FOR SAMPLES FROM ANTIPODES ISLAND.........61

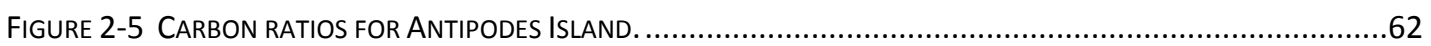

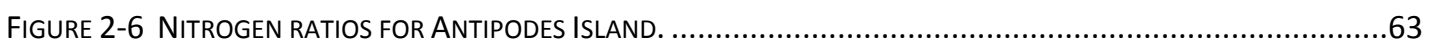

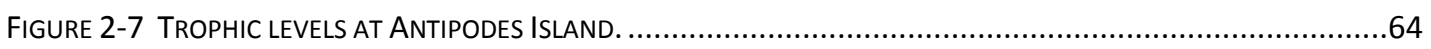

FIGURE 2-8 PLOT OF CARBON AND NITROGEN STABLE ISOTOPE RATIOS FOR SAMPLES FROM THE BOUNTY ISLANDS. ......65

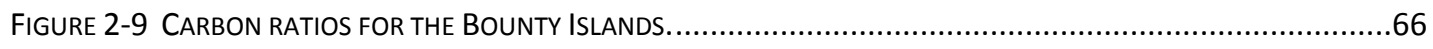

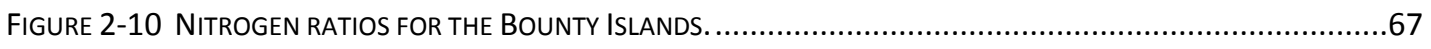

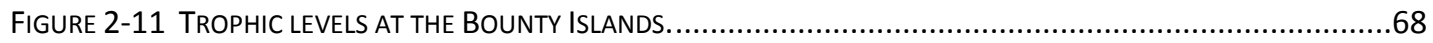

FIGURE 3-1 MAP OF WATER NUTRIENT AND PHYTOPLANKTON STABLE ISOTOPE SAMPLING SITES IN THE OPEN OCEAN AND ON 12 NAUTICAL MILE TRANSECTS AT ANTIPODES AND THE BOUNTY ISLANDS.

FIGURE 3-2 WATER NUTRIENT SAMPLING AND PHYTOPLANKTON STABLE ISOTOPE SAMPLING SITES AT ANTIPODES ISLAND.

FIGURE 3-3 WATER NUTRIENT SAMPLING AND PHYTOPLANKTON STABLE ISOTOPE SAMPLING SITES AT THE BOUNTY ISLANDS.

FIGURE 3-4 WATER NUTRIENT CONCENTRATIONS RECORDED ON TRANSECTS AND AT COASTAL SITES AROUND ANTIPODES

ISLAND.

FIGURE 3-5 WATER NUTRIENT CONCENTRATIONS RECORDED ON TRANSECTS AND AT COASTAL SITES AROUND THE

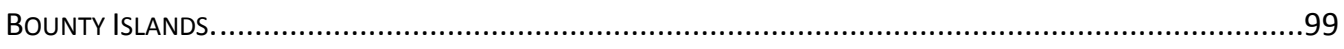

FIGURE 3-6 WATER NUTRIENT CONCENTRATIONS RECORDED AT OPEN OCEAN SITES. .................................. 100

FIgURE 3-7 PHYTOPLANKTON STABLE ISOTOPE TRANSECT AT ANTIPODES ISLAND ........................................ 101

FIGURE 3-8 PHYTOPLANKTON STABLE ISOTOPE TRANSECT AT THE BOUNTY ISLANDS....................................... 101

FIGURE 3-9 PHYTOPLANKTON STABLE ISOTOPE SIGNATURES FROM OPEN OCEAN SITES. ................................... 102

Figure 3-10 ChLorophyLl a MAP Of THE CAMPBELL PLATEAU APRIL 2009, fROM MODIS........................... 113

FIGURE 3-11 PARAKEET FORAGING IN PENGUIN GUANO AT ANTIPODES ISLAND. ............................................ 114

FIGURE 3-12 BURROW OF THE SPIDER PACIFICANA, CONSTRUCTED FROM FUR SEAL FUR ON THE BOUNTY ISLANDS... 115

FIGURE 4-1 ANTIPODES ISLAND FOOD WEB SHOWING THE CONNECTIONS BETWEEN SEABIRD AND MARINE MAMMAL FORAGING SITES, THE NEARSHORE MARINE ENVIRONMENT AND TERRESTRIAL ENVIRONMENTS..................... 128

FIGURE 4-2 BOUNTY ISLANDS FOOD WEB SHOWING THE CONNECTIONS BETWEEN SEABIRD AND MARINE MAMMAL FORAGING SITES, THE NEARSHORE MARINE ENVIRONMENT AND TERRESTRIAL ENVIRONMENTS. .... 


\section{List of Tables}

TABLE 2-1 LIST OF SAMPLES COLLECTED FROM ANTIPODES ISLAND INCLUDING STORAGE, TREATMENT AND STABLE ISOTOPE SIGNATURES..

TABLE 2-2 LIST OF SAMPLES COLLECTED FROM THE BOUNTY ISLANDS INCLUDING STORAGE, TREATMENT AND STABLE ISOTOPE SIGNATURES.

TABLE 2-3 PhySICAL hABITATS RECORDED AT ANTIPODES AND BOUNTY ISLANDS DURING MARCH 2009. .................44

TABLE 2-4 COMPARISON OF CARBON AND NITROGEN RATIOS AT ANTIPODES ISLAND SITES.................................59

TABLE 2-5 COMPARISON OF CARBON AND NITROGEN RATIOS AT BOUNTY ISLANDS SITES. ...................................59

TABLE 2-6 COMPARISON OF CARBON AND NITROGEN RATIOS BETWEEN THE BOUNTY AND ANTIPODES ISLANDS..........60

TABLE 3-1 LIST OF WATER NUTRIENT CONCENTRATIONS COLLECTED FROM ANTIPODES ISLAND. ...........................92

TABLE 3-2 LIST OF WATER NUTRIENT CONCENTRATIONS COLLECTED FROM THE BOUNTY ISLANDS...........................93

TABLE 3-3 LIST OF WATER NUTRIENT CONCENTRATIONS COLLECTED FROM THE OPEN OCEAN................................94

TABLE 3-4 LIST OF PHYTOPLANKTON STABLE ISOTOPE VALUES COLLECTED FROM ANTIPODES ISLAND........................95

TABLE 3-5 LIST OF PHYTOPLANKTON STABLE ISOTOPE VALUES COLLECTED FROM THE BOUNTY ISLANDS. ....................95

TABLE 3-6 LIST OF PHYTOPLANKTON STABLE ISOTOPE VALUES COLLECTED FROM THE OPEN OCEAN ..........................96

TABLE 3-7 CORRELATION BETWEEN DISTANCE AND NUTRIENT CONCENTRATIONS AND PHYTOPLANKTON STABLE ISOTOPES

(PEARSON CORRELATION COEFFICIENTS) AT THE ANTIPODES AND BOUNTY ISLANDS ...................................96

TABLE 3-8 FRACTIONS OF KELP, PHYTOPLANKTON (PHYTOPL.) AND TERRESTRIAL CARBON IN CONSUMERS AT THE

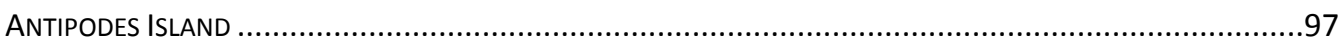

TABLE 3-9 FRACTIONS OF KELP AND PHYTOPLANKTON IN CONSUMERS' DIETS AT THE BOUNTY ISLANDS .....................98 


\section{CHAPTER ONE: General Introduction}

One of the primary objectives of ecology is to determine the patterns of energy flow, nutrient cycling and how food webs within populations, communities and ecosystems are constructed (Rounick and Winterbourn, 1986). In recent years, understanding the functional importance of species interactions within and between ecosystems has become increasingly important for finding ecosystem-based solutions to the conflict between human use and biodiversity (Jabiol et al., 2013). But what constitutes an ecosystem? An ecosystem can be described as "a spatially explicit unit of the earth that includes all of the organisms, along with all the abiotic environment within its boundaries” (Likens, 1992). This definition implies that that an ecosystem is scale dependant (Hoekstra, 1992) and that the scale of an ecosystem is determined by the organisms, the physical environment and the interactions that exist within it (Pickett and Cadenasso, 2002).

There are inherent difficulties in determining the boundaries of an ecosystem, in particular where the processes of interest are operating at different scales (Post et al., 2007a) and because processes may be simple, stable, or changing in composition (Holling, 1973). Therefore, in order to bring the conceptual idea of an ecosystem into reality, the definition of an ecosystem needs to be translated into useable tools to explain the components, interactions and the drivers of an ecosystem (Pickett et al., 2010).

\subsection{ECOSYSTEM AND FOOD WEB MODELS}

Models are valuable tools to help describe complex ecological interactions, and how energy is processed, partitioned and dissipated within ecosystems (Pickett and Cadenasso, 2002). There are a range of ecosystem models that focus on components of an ecosystem, based on the energy flows, the nutrients that drive an ecosystem and the trophic interactions between organisms. Ecosystem models can range from 
trophic models such as those developed using Ecopath (McClanahan and Sala, 1997, Heymans and Baird, 2000, Pauly et al., 2000, Bradford-Grieve et al., 2003) that use a combination of input parameters such as biomass and diet, to those that are more simple and based around hypotheses regarding how a ecosystem is structured and functions. An example of a more "simple" model is the trophic cascade model developed for temperate north eastern New Zealand kelp forests, that describes the interactions among predators, their prey and kelp (Shears and Babcock, 2002, Babcock et al., 1999).

Food web models are one of the most common tools for describing and assessing the energy flow and relationships between species and are useful for providing management context (Link, 2002). Food webs are also useful for comparing marine systems between sites. For example, Jacob et al. (2006) used a food web model to describe the trophic functioning of Bouvet Island in the Southern Ocean, and found that the system was dominated by omnivory and was therefore similar to other Antarctic systems. That study also determined that the Bouvet Island benthic and pelagic systems were almost entirely linked to pelagic primary production. In a similar study, Kaehler et al. (2000) described the trophic pathways of the Prince Edward Islands food web to determine the relative importance of different sources of organic matter in supplying the food web. These authors found that in contrast to the Bouvet Island system, there was a clear pattern for most of the species within the food web to be consuming kelp-derived organic matter.

Analysis of food web structure is useful in addressing not only the structure and functioning, but also the stability of ecosystems. McMeans et al. (2013) used a food web to describe resource utilisation by consumers in a highly variable Arctic ecosystem and found that the food web was structured around separate basal carbon sources (phytoplankton and macroalgae) and suggested that heterogeneity is the basis of a stable food web. They also suggested that the loss of heterogeneity may be the greatest threat to food web stability. 
Within the last decade, food web studies have started to move from studies at localised spatial scales to exploring the concept of connectivity between ecosystems and the role that resource subsidies may play in the functioning of both terrestrial and marine food webs (Marczak et al., 2007). For example, a number of studies have shown that nutrient input from the marine environment directly and indirectly subsidises terrestrial environments (Polis and Hurd, 1995, Polis and Hurd, 1996, Anderson and Polis, 1998, Barrett et al., 2005). The introduction of nutrients to terrestrial environments from sea birds also reticulates back into the marine environment, altering the levels of sea water nutrient concentrations and enhancing primary production (Zwolicki et al., 2012, Kolb et al., 2010, Golovkin, 1967, Wainright et al., 1998). Whilst considerable progress has been made in understanding the structure and functioning of particular ecosystems, the drivers behind them and the direct and indirect subsidisation of receiving environments, little progress has been made in combining these methods to inform management.

In recent years, there has been a focus by decision makers to move from a speciesbased management framework, where species are managed largely in isolation from each other, to an ecosystem-based management (EBM) framework. EBM can broadly be described as an integrated management approach that considers the entire ecosystem, including all linkages and the cumulative impacts of human-induced stressors (Browman et al., 2004). There are a number of management tools that are currently used to attempt to implement the concept of ecosystem based management, such as marine spatial planning, marine protected area planning processes and ocean zoning (Toonen et al., 2011). Whilst EBM is a logical progression from managing the direct impact of activities on species to the wider impact on ecosystem functioning, there are still considerable challenges in implementing an EBM approach due to the limited availability of ecosystem-scale data and the largely sector-by-sector management approach by management agencies (Samhouri and Levin, 2012). 


\subsection{STABLE ISOTOPE ANALYSIS}

Stable isotope analysis is becoming an increasingly popular tool for investigating the trophic interactions within marine communities (Gannes et al., 1998, Rounick and Winterbourn, 1986, Peterson and Fry, 1987). It is a useful tool for investigating not only the diet and energy sources of particular species (Peterson et al., 1985), but also for understanding their migratory patterns and feeding locations (Kurle and Worthy, 2002). Stable isotope analysis can therefore be a useful method for constructing food webs, understanding ecosystem structure and function, and informing ecosystem-based approaches to management. It can be particularly useful in the marine environment to construct food webs where a broad collection of species can be made in a short sampling period, if environmental conditions, timeframes or cost are a constraint (Michener and Lajtha, 2007).

A number of food web studies have used naturally occurring stable carbon and nitrogen isotope ratios to study the trophic interactions between consumers and prey (Fry et al., 1982, Peterson et al., 1985, Hobson and Welch, 1992, Kwak and Zedler, 1997, Wainright et al., 1993, Kaehler et al., 2000, Nyssen et al., 2002, Harding et al., 2004, Christensen and Richardson, 2008, Lesage et al., 2010, McMeans et al., 2013). These studies have shown that under certain circumstances, isotopes show a stepwise enrichment between the tissues of consumer and prey (Minagawa and Wada, 1984, Mizutani and Wada, 1988, Fry and Sherr, 1984). The isotopic composition of a consumer's diet is assimilated into its tissues by digestion, absorption, respiration and excretion. Therefore, by the measurement of a consumer's prey, the consumer can be assigned to its trophic position. However, in order to fully utilise the potential of stable isotope analysis in field studies, there is a requirement for prior knowledge of the potential prey items of predators (Vander Zanden and Rasmussen, 2001).

The physiological processes in the tissue of a consumer often occur at different rates for compounds that contain light in contrast to heavy isotopes of carbon (12C 
compared to 13C) and nitrogen (14N compared to $15 \mathrm{~N}$ ) due to the differences in their chemical masses (Fry, 2006, Phillips, 2012). This difference is referred to as isotopic fractionation (Peterson and Fry, 1987), tissue discrimination (Miller et al., 2008), trophic shift (McCutchan et al., 2003), or trophic enrichment (Hobson and Welch, 1992). The ratio of stable isotopes of nitrogen $\left(\delta^{15} \mathrm{~N}\right)$ can be used to determine the trophic position of consumers relative to their prey, as the consumer is typically enriched by as much as $+3-4$ \%o relative to their prey (Peterson and Fry, 1987, Minagawa and Wada, 1984). Carbon isotopes $\left(\delta^{13} \mathrm{C}\right)$ on the other hand, undergo much smaller changes of up to $+1 \%$ between consumer and prey and can be used to evaluate the source of carbon through food webs, i.e. a consumer should have a similar carbon signature to its prey (Post, 2002).

Island ecosystems offer opportunities for studying ecosystem processes and have been recognized as natural laboratories, due to their discrete geographical nature and diversity of species and habitats (Emerson, 2002). Island ecosystems have been suggested as suitable models for the study of ecosystems (Vitousek, 2002), including aspects such as cross-ecosystem nutrient transfer and the definition of the boundaries of ecosystems (Polis and Hurd, 1996). Oceanic islands in particular may be a useful tool for studying ecosystem connectivity, due to their importance for air-breathing species such as seabirds and pinnipeds that need to utilise terrestrial environments for resting, moulting, mating and rearing young, but that also rely on the islands' surrounding waters for food (Chown et al., 2001). The spatial and temporal scale of these interactions is important for defining the extent of an ecosystem and may be useful for informing management of island ecosystems (Wienecke and Robertson, 2002). Oceanic islands are also often of conservation significance and can be particularly vulnerable to anthropogenic threats (Chown et al., 1998), increasing the importance of understanding how these systems are structured and function. 


\subsection{SUBANTARCTIC REGION}

The subantarctic region lies north of the Antarctic Convergence and south of the Subtropical Convergence. There are twenty major islands or island groups, including islands in the southern Indian, Pacific and Atlantic Oceans (Peat, 2003). Many of these islands have high conservation value, particularly due to their pronounced endemicity and seabird diversity (Chown et al. 2001; Clark and Dingwall 1985), The marine environments associated with these islands remain largely undescribed, due primarily to the logistical issues associated with conducting marine research in such isolated areas (Booth, 2004). Understanding the structure and function of these ecosystems is important for increasing our understanding of the biodiversity at risk from potential threats and how best to manage these areas (Freeman et al., 2011).

New Zealand's subantarctic islands, the Auckland Islands, Campbell Island, Antipodes Island and the Bounty Islands, are located on Campbell Plateau, a submerged portion of the New Zealand continental landmass off the south and eastern coasts of New Zealand. They occupy the stormy latitudes of the Roaring Forties and Furious Fifties, a transition zone between mainland New Zealand and Antarctic regions (Department of Conservation, 2006). Together with Macquarie Island (Australian territory) these are the only subantarctic islands in the Pacific sector of the Southern Ocean. The New Zealand Subantarctic Islands have 126 bird species in total, including 40 seabirds, of which five breed nowhere else in the world (Department of Conservation, 1998). New Zealand fur seals and elephant seals also breed in large numbers at the islands. A review by Booth (2004) suggested a potentially high level of difference in biodiversity among the islands and also that all four island groups have high levels of endemism in some taxa. However, there is currently little quantitative data to verify this statement.

New Zealand's Subantarctic Islands and the surrounding plateau is covered by Subantarctic Water (SAW) of uniform properties and bounded on all sides by major oceanic fronts. Typically, the surface temperatures are $11^{\circ} \mathrm{C}$ in summer and $7^{\circ} \mathrm{C}$ in 
winter, with surface salinities of about 34.5 ppt (Morris et al., 2001). The Subantarctic Islands have a European history extending over 200 years including discovery, sealing, whaling, scientific exploration, colonisation and settlement, farming, shipwrecks of the sailing era, wartime coastwatching, research and meteorology (Peat, 2003). European discovery of the island groups occurred over a period of 21 years: 1788-1810. Following each discovery, there was a large but short lived sealing boom at the islands resulting in a collapse of seal populations at all the islands (Laws, 1994, Taylor and Taylor, 1989, Taylor, 1996). There have therefore been some significant changes to the terrestrial and marine biodiversity of the islands.

Very few marine scientific expeditions have been undertaken to the New Zealand Subantarctic Islands. The connectivity between the islands and their surrounding seas is complex (Department of Conservation 2006), but the lack of data on the structure and function of the islands' marine communities have not allowed an assessment of the nature of these trophic relationships and the biotic and abiotic drivers. The intertidal biota (Hayward and Morley, 2005), fish fauna (Clark, 1985, Kingsford et al., 1989) and marine algae (Hay et al., 1985) of the Campbell and Auckland Islands have received some attention, although there are still significant areas requiring further study. Marine species recorded from the Bounty and Antipodes Islands have been included in studies of the biogeography and genetic connectivity of particular taxa (Forest and McLay, 2001, Goldstien et al., 2009) and there are also published inventories for some marine taxa (Clark, 1971, Hay et al., 1985). However, the only previous scientific accounts of the nearshore marine communities of the Bounty and Antipodes Islands are qualitative descriptions of species and species assemblages observed by divers during an expedition in 1978 (Horning, 1986). A large number of collections were made during that expedition, making a significant contribution to knowledge of the marine biodiversity of the islands. More recently, Freeman et al. (2011) described the nearshore community structure at the Antipodes and Bounty Islands and suggested that the islands, despite being only 100 nautical miles apart have very different benthic communities, but did not address the reasons for the differences. 


\subsection{STUDY SITES}

The two New Zealand Subantarctic Island groups that comprise my study sites are the Bounty Islands and Antipodes Island (Figure 1-1).

\subsubsection{BOUNTY ISLANDS}

The Bounty Islands are located $700 \mathrm{~km}$ east-south-east of New Zealand. They are of early Jurassic age (around 177-188 million years old) and are formed of coarse granite (Adams and Cullen, 1978). The total land area of the Bounty group is $135 \mathrm{ha}$, consisting of 20 small islands in three distinct clusters (main, centre and east). The largest, Depot Island is $800 \mathrm{~m}$ long and reaches a maximum altitude of $88 \mathrm{~m}$ (Figure 1-2). There is no soil on the islands but they are covered by a layer of guano and scat formed by birds and marine mammals (Booth, 2004). The Bounty Island Cook's Scurvy grass is the only vascular plant that exists on the islands and is known from only a few plants at one site (Amey et al., 2007). The islands support the largest breeding population of New Zealand fur seals, estimated at over 20,000 (Taylor, 1996). Seven species of bird breed at the islands, including large numbers of erectcrested penguins and Salvin's albatross (Robertson and van Tets, 1982). Other birds include the endemic Bounty Island shag, a species of fulmar prion, cape pigeons, Antarctic terns and black-backed gulls (Department of Conservation, 1998).

The underwater features of the Bounty Islands consist of large, steep walls, boulders and highly pinnacled areas. The surrounding ocean is an extremely high energy marine environment and no sheltered areas exist around the group - every island is prone to very heavy seas on a regular basis. Very little is known about the inshore ecosystem of the Bounty Islands due to their remote nature and difficult working conditions. Recent marine expeditions to the group have shown that the marine 
environment has considerable marine diversity. Freeman et al. (2011) described the Bounty Islands as having thickly encrusted benthic communities with a range of filter and suspension-feeding invertebrates, which included sponges, ascidians, barnacles, mussels and bryozoans. Few coralline algae were observed at the Bounty Islands, and the highly abundant plate corallines observed at Antipodes Island were absent from the Bounty Islands.

\subsubsection{ANTIPODES ISLAND}

Antipodes Island is situated on the eastern margin of the Bounty Plateau, $100 \mathrm{~nm}$ from the Bounty Islands and $820 \mathrm{~km}$ southeast of New Zealand's South Island - they are the most remote of all the New Zealand Subantarctic Islands (Peat, 2003). The main island, which is around 2000 hectares in area, consists of the remains of a Pleistocene volcano and basalt boulders and columns are common around the island (Knox, 1987) (Figure 1-3). The island is exposed to persistent westerly winds, often gale force, and between $1000 \mathrm{~mm}$ and $1500 \mathrm{~mm}$ of rain falls each year (Department of Conservation, 1998).

Freeman et al. (2011) described the Antipodes Island subtidal rock wall communities as dominated by nongeniculate coralline algae that form extensive areas of intricate plated structures. The only other locations in New Zealand recorded as having such extensive areas of plate-forming nongeniculate coralline algae are Campbell Island and the Auckland Islands. Encrusting invertebrates are also common at Antipodes Island, with bryozoans, anemones and sponges being particularly abundant. Complex three-dimensional spaces are provided by the algae, and a range of mobile invertebrates, along with thornfish, appeared to use these plating structures as refuges.

The coastline of Antipodes Island is comprised of many steep-sided islets and stacks. Boulder beaches are present with a number of large caves scattered around the island. Sheers cliffs rise up to $150 \mathrm{~m}$ from the ocean floor and tower above the 
island with wave platforms cut out of the rock in a few areas. These attributes form a varied and diverse range of habitats for the marine flora and fauna to inhabit (Department of Conservation, 1998). The predominant coastal vegetation is tussock grassland with ferns, four species of Coprosma and the herb Stilboccarpa polaris. Twenty five species of bird breed on the island with rockhopper and erect-crested penguins being the most abundant (Warham and Bell, 1979). Wandering albatross, light-mantled sooty albatross, northern giant petrel, white chinned petrel and southern skua are all common on the island (Department of Conservation, 1998). There are four species of endemic terrestrial birds at the islands - the Antipodes Island snipe, pipit, Antipodes parakeet and Reischek's parakeet (red-crowned parakeet). Fur seals are present at the islands in increasing numbers after being decimated during the sealing years. It has been estimated that up to 330,000 seals were taken from these islands over a period of 20 years in the 1800's (Taylor, 2006). Elephant seals also breed in low numbers at the island, however little information exists on their population status (Peat, 2003).

\subsubsection{CURRENT MANAGEMENT}

All of New Zealand's subantarctic islands were afforded the highest level of terrestrial protection, as national nature reserves in 1978. The Territorial Sea surrounding the Auckland Islands is also protected by a no-take marine reserve and marine mammal sanctuary. The Subantarctic Islands were recognised for their significant conservation value and designated as World Heritage Areas in 1998. Large Benthic Protected Areas provide partial protection to benthic species through the exclusion of bottom trawling and dredging within $100 \mathrm{~m}$ of the seabed (Ministry of Fisheries 2007) but may not be optimally placed in terms of benefits to conservation and costs to the commercial fishing industry (Leathwick et al. 2008). For the purposes of implementing additional marine protected areas in the New Zealand Territorial Sea, a coastal marine classification was developed (Ministry of Fisheries and Department of Conservation 2008), which placed all New Zealand's Subantarctic Islands in one biogeographic region. A process to consider marine 
protected areas in the Subantarctic Biogeographic Region was formally initiated in 2008 (Subantarctic Marine Protection Planning Forum 2009). The Forum provided options for protected areas to government in 2011 for consideration. The Minister of Conservation and Minister for Primary Industries agreed to proceed with a protection package that is currently awaiting finalisation by way of special legislation.

\subsection{THESIS AIMS}

I hypothesise that that due to each island's isolation, contrasting terrestrial ecology, oceanic position and the abundance of sea birds and marine mammals, that the islands will have differing food webs and that there will be strong links between the marine and terrestrial environments. The primary aim of this thesis is to describe and compare the food webs of Antipodes Island and the Bounty Islands. In Chapter Two I will use stable isotopes to determine the trophic structure of each island group. In Chapter Three I will identify the drivers of each food web by determining the basal carbon sources and nutrient concentration status in the islands' surrounding waters. I will also use mixing models to determine the diets of key components of the islands' ecosystems, and to identify if there are any trophic linkages between the marine and terrestrial realms. In my general discussion I will propose food web models for each island group and discuss some of the management implication of these. 
Figure 1-1 Map showing location of the Bounty Islands and Antipodes Island.

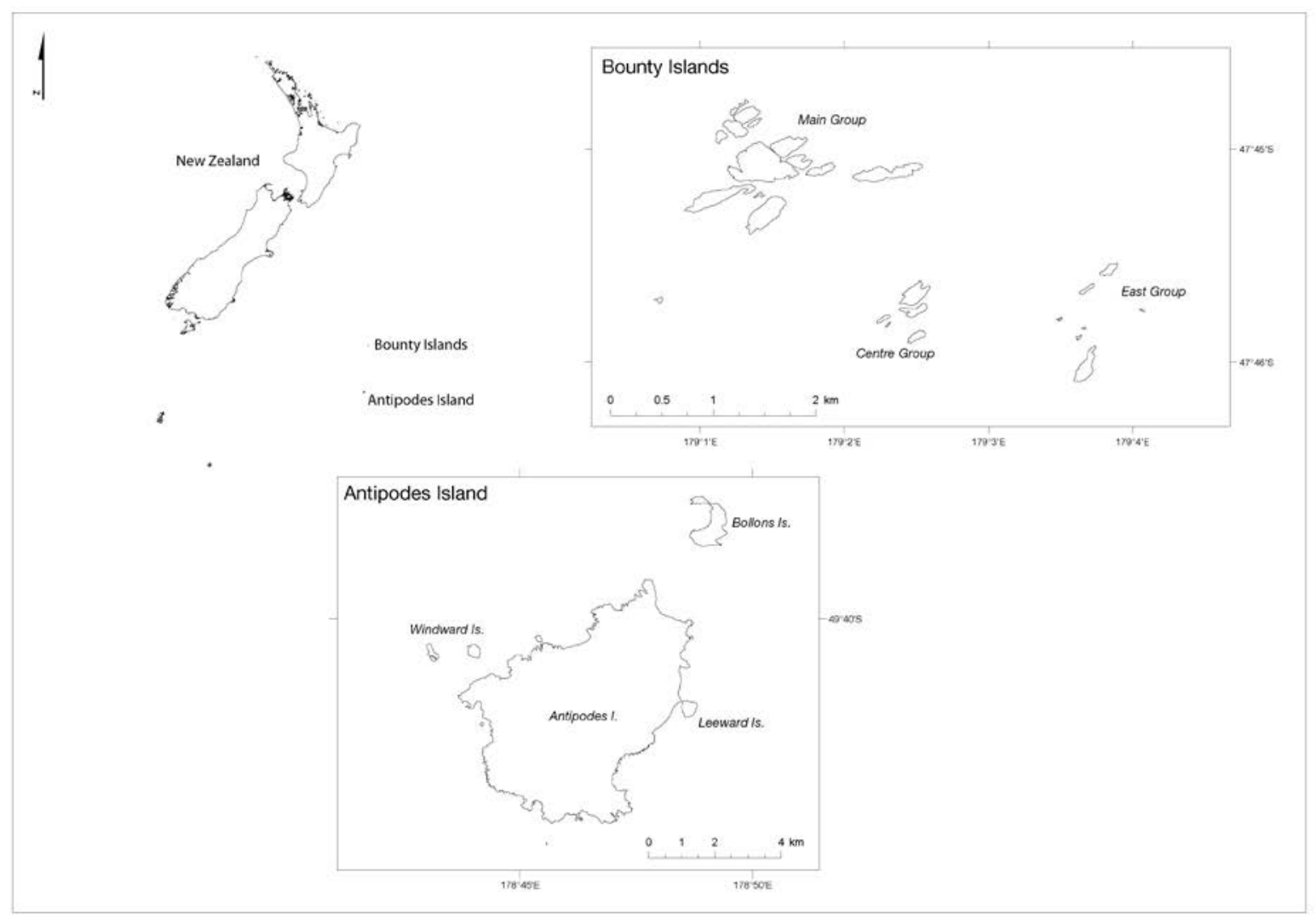


Figure 1-2 Depot Island, the Bounty Islands, demonstrating the abundance of seabirds (erectcrested penguins and Salvin's albatross) on the islands.

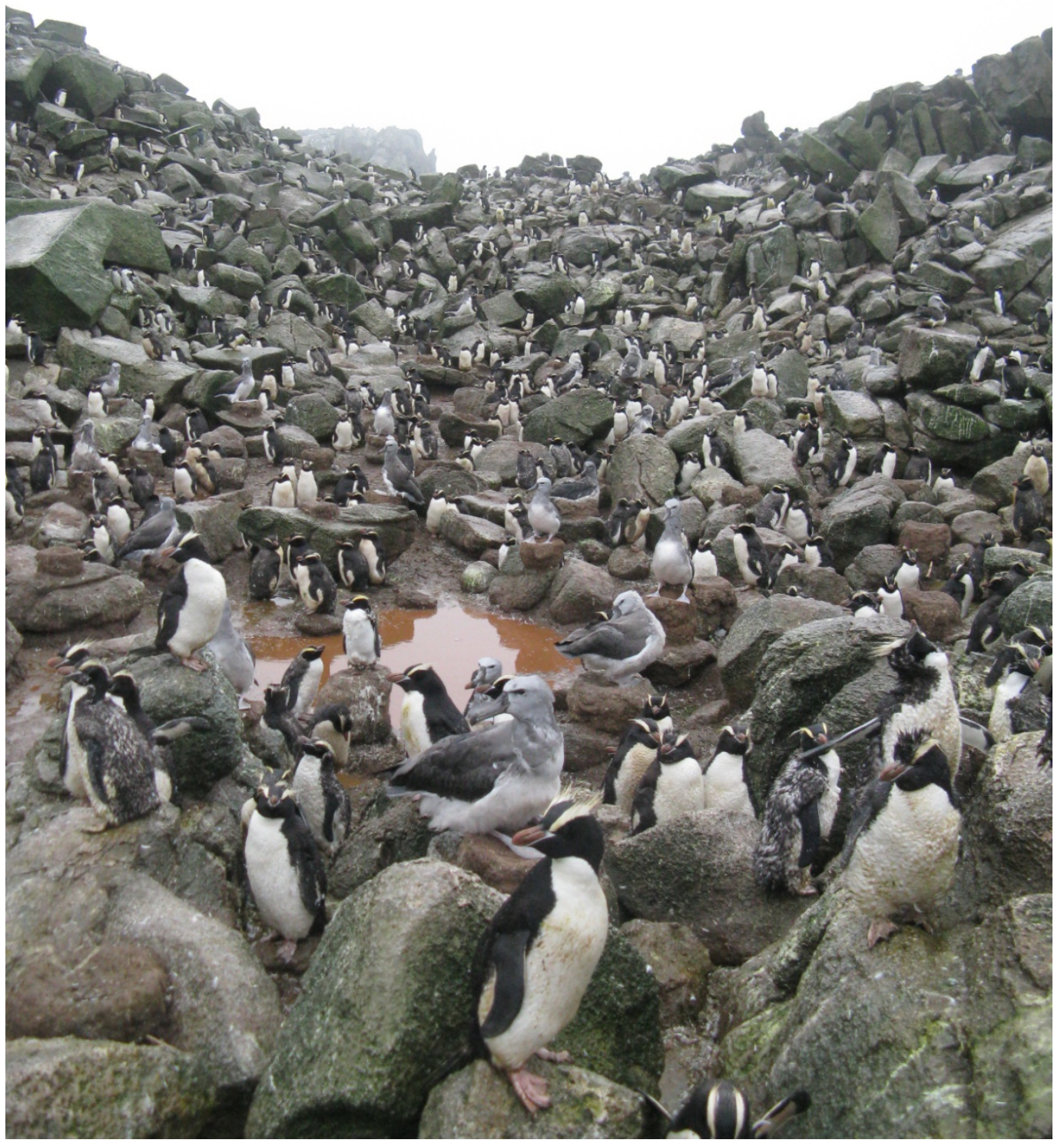


Figure 1-3 Antipodes Island, with penguin colony on the slope leading up to Mount Waterhouse.

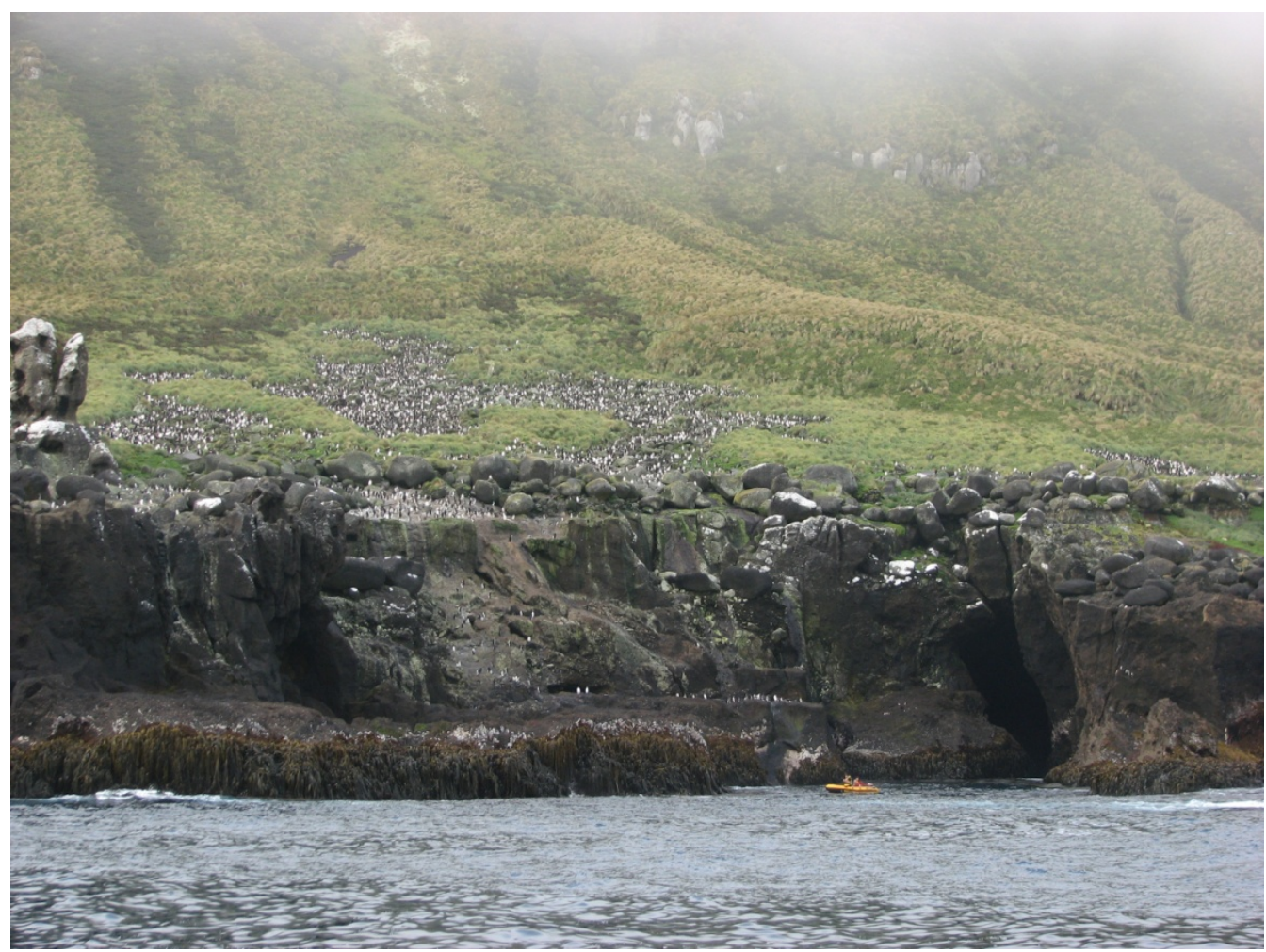




\section{CHAPTER TWO: Trophic Structure at the Bounty and Antipodes Islands}

\subsection{INTRODUCTION}

Understanding the structure and functioning of ecosystems requires information on the trophic relationships of key species (Sydeman et al., 1997). Food webs are a valuable and structured way to determine the importance of the trophic linkages that determine ecosystem dynamics (Link, 2002). The construction of food webs is essential in understanding how an ecosystem functions and to provide context for environmental management (Crowder et al., 1996, Polis and Winemiller, 1996). Food webs are ultimately linked to the resources that are available to sustain them and in the case of the coastal marine environment, the base of the food web are primary producers, either being phytoplankton or macroalgae detritus (Polis et al., 1996); there are also bacterial-based food webs for which there is little information (Pinkerton et al., 2008, Lundquist and Pinkerton, 2008). The flux of these resources may influence the structure and stability of food webs, especially when they fluctuate seasonally (Norkko et al., 2007) or where human disturbance influences energy flow (Mumby et al., 2012). Rooney et al. (2006) suggest that food webs that are based on the maintenance of heterogeneity of energy channels in a system will be more resilient to disturbance. It is therefore important for ecologists and environmental mangers to identify the components of an ecosystem that allow the processes, resilience and overall stability in food webs to be maintained (Rooney et al., 2006).

In recent years, the understanding of food webs has increased and hence our knowledge of the complexity of food webs has also increased. Determining trophic levels has been one of the most common ways in ecology to gain an understanding of energy flow and top down control within food webs (Thompson et al., 2007). 
Trophic levels provide a simple way to view the energy links within a food web by organising taxa into groups that reflect their trophic position i.e what species are consuming. Research on trophic levels is centred around patterns that are common within all ecological systems (Lindeman, 1942, Pimm and Lawton, 1978, Martinez and Lawton, 1995), the patterns that characterise particular types of systems (Hairston and Hairston, 1997, Polis and Strong, 1996) and patterns that characterise the role of species in systems (Brett and Goldman, 1997, Schmitz et al., 2000). Trophic position represents the number of feeding links that separate a species from the base of production (Thompson et al., 2007). A trophic level (TL) that is greater than or close to 1 indicates a primary producer or a grazing species; between 1 and 2 indicates an omnivore or predatory invertebrate; and greater than 3 indicates a top level predator. These TL integers have been used extensively (Zanden and Rasmussen, 1999, Jack and Wing, 2011, McMeans et al., 2013, Hobson et al., 1995, Kaehler et al., 2000).

Small oceanic islands are ideal model ecosystems for undertaking ecological studies, due the island communities being spatially restricted and often largely free from human disturbance (Whittaker and Fernández-Palacios, 2007). Island ecology research has mostly focused on the terrestrial environment whilst the marine environment has been comparatively poorly studied (Martins et al., 2008). While often little is known regarding how island marine ecosystems function, they provide an opportunity to study discrete populations that may yield answers to ecological questions regarding the structure and functioning of ecosystems that could be applied to more complex mainland systems (Wardle, 2002).

Food webs in the coastal environment are complex and change both temporally and spatially (Paine, 1988) making the study of trophic interactions at remote sites particularly problematic. Traditional trophic and dietary studies have relied on stomach and scat content analysis to determine the trophic position of species within ecosystems, but this only provides a snapshot in time of ingested food (Michener and Lajtha, 2007, Fry, 2006). These methods yield mostly qualitative results 
(Vander Zanden and Rasmussen, 2001) and may be limiting as the assimilated diet of consumers over time is not captured (Hobson and Welch, 1992, Phillips, 2001, Post, 2002, Fredriksen, 2003). In the last few decades there has been considerable interest by ecologists in using stable isotopes as an alternative tool for studying trophic interactions and addressing questions regarding food webs and ecological studies (Peterson and Fry, 1987). Stable isotope analysis has distinct advantages over traditional methods, as the information yielded provides both trophic level and food web data (Post et al., 2007b, Owens, 1988). Also, traditional studies are not effective for tracking mass energy flow through ecological communities (Paine, 1988, Post et al., 2007b, Zanden and Rasmussen, 1999), which may have implications for their utility in developing trophic models.

Stable isotopes can provide important information for determining food web architecture, for example, stable isotopes of nitrogen $\left(\delta^{15} \mathrm{~N}\right)$ can be used to determine the trophic position of consumers relative to their prey, as the consumer is typically enriched by as much as $+3-4$ \%o relative to their prey (Peterson and Fry, 1987, Minagawa and Wada, 1984). Carbon isotopes $\left(\delta^{13} \mathrm{C}\right)$ on the other hand, undergo much smaller changes of up to $+1 \%$ between consumer and prey and can used to evaluate the source of carbon through food webs where the $\delta^{13} \mathrm{C}$ signature of the prey differs from the consumer (Post, 2002); this provides information on what species are consuming. Stable isotopes can also allow for non-invasive and nonlethal sampling of species to determine diet, which may be particularly useful when studying protected species or protected areas (Willis et al., 2013). Stable isotopes also provide a potentially powerful tool for the study of remote island ecosystems, or where year round sampling may be constrained by environmental conditions or where the sampling is limited by particular species' breeding seasons (Hobson, 1993).

The Antipodes and Bounty Islands are largely pristine and protected in National Nature Reserves, both containing large concentrations of protected species (Chown et al., 2001). Stable isotope analysis is appropriate for this study to determine the 
islands' food webs because of the prevalence of protected species and because stable isotopes can provide a time-integrated measure of energy flow through communities, trophic pathways of consumer and prey, and capture the potential complex interactions between species that reflect the long term assimilation of a consumer's diet (Jacob et al., Michener and Lajtha, 2008).

In the New Zealand Subantarctic Islands, recent research by Freeman et al. (2011) identified significant differences in the benthic marine communities between Antipodes Island and the Bounty Islands - those found at the Antipodes were more diverse and had higher abundance than the Bounty Islands, and the functional groups represented at the islands were different. These findings raise the question of how these differences are reflected in the food web structure at the islands and what this means for the stability and resilience of island ecosystems.

In this chapter, the isotopic composition and the trophic interactions of species at Antipodes and the Bounty Islands will be determined using stable isotope signatures of marine and terrestrial species. I aim to establish the trophic level of species at the islands and take the first steps towards characterising their trophic structure and identifying any differences between the islands. I will use this information to assess if the island ecosystems are currently stable and explore how resilient they may be to disturbance such as environmental change and removal of top predators. 


\subsection{METHODS}

In March 2009, a boat-based scientific expedition was undertaken to survey the biodiversity of the remote Antipodes Island and Bounty Islands (Figure 2-1).

\subsubsection{SITE SELECTION}

In order to describe the trophic interactions at Antipodes Island and the Bounty Islands, haphazard sampling was employed to collect a broad range of marine and terrestrial taxa that represented the most common and highest biomass of each island (Freeman et al., 2011). Marine samples were collected from subtidal habitats by SCUBA divers. Sampling was undertaken from $0 \mathrm{~m}$ to $30 \mathrm{~m}$ depth, mostly on rocky reef habitat but also on soft sediment habitats. Intertidal sampling was carried out by snorkelling or by SCUBA on rock walls and by hand at low tide on wave platforms and boulder beaches. Terrestrial samples were collected from the coastal zone of the Bounty Islands and on the Northern Plains of Antipodes Island within areas with high densities of top level predators such as seabirds and marine mammals and also in areas that either had no top level predators present or where there were low numbers. Terrestrial samples were collected by hand and using traps (for mice). The Bounty Islands are virtually vegetation-free, and so no terrestrial plants were collected from this location.

Sampling site selection at each island (Figure 2-2) was based on the prevailing weather conditions, where divers could safely enter and exit the water and where attending vessels could operate. Terrestrial sites where chosen on the basis of the ability to land and undertake sampling safely. 


\subsubsection{SAMPLING}

Tissue samples were collected from macroalgae, small invertebrates, terrestrial plants, fish, seabirds, marine mammals and introduced terrestrial mammals (mice) (Table 2-1, Table 2-2). Seabirds were captured by hand and had feathers clipped from both the breast and wings. Fur and skin samples were taken from New Zealand fur seals and elephant seals. Fur seal tissue was obtained from fur seal pups at the Bounty Islands using piglet ear-notching pliers, removing a small (approximately $5 \times 5 \mathrm{~mm}$ ) segment of flipper tissue. Flipper clips from suckling pups are known to have similar isotopic composition to their mothers (Hobson et al., 1997b). At Antipodes Island, fur seal fur was scraped off rocks within haul out sites - no live adults or pups could be captured at Antipodes Island due to their wariness of our presence. At Antipodes Island, elephant seal tissue samples were collected using a long biopsy pole (no elephant seals occur at the Bounty Islands). Scat and guano samples were collected by hand from each island. Due to the threatened and protected status of the terrestrial and sea birds at each island, samples were where possible taken from recently-deceased individuals or from moults to minimise impacts on these species (Vasil et al., 2012).

Where possible, replicates of least three individuals per species were collected and stored separately in plastic bags during each dive. Intertidal and terrestrial species were also sorted in the same manner prior to processing. Table 2-3 provides a list of the habitats sampled at the Bounty and Antipodes Islands.

Plankton sampling was undertaken by filtering seawater collected from stations in the open ocean and at intervals of 2.5 nautical miles along two transects completed at each island when entering and exiting the territorial seas of each island (Figure 2-3). For further details of phytoplankton sampling see Chapter Three. 


\subsubsection{STORAGE OF SAMPLES}

Sample storage was determined by tissue type. Macroalgae were sorted by

individual species and tissue from each species was stored in a freezer $\left(\right.$ at $\left.-20^{\circ} \mathrm{C}\right)$ and then later in the expedition in 70\% ethanol due to the limited space available in the vessel's freezer. Terrestrial plants were stored frozen. A few grams of muscle tissue of larger invertebrates and fish was dissected out, but smaller invertebrates were stored whole - all in 70\% ethanol. Marine mammal fur and scats were stored fresh in containers and plastic bags. Marine mammal skin samples were stored in vials containing 70\% ethanol. Bird feathers and dried tissue (from deceased birds) were stored fresh in plastic bags. Mice were caught in mouse traps; the tail tips of the mice were removed and stored in vials containing $70 \%$ ethanol.

\subsubsection{SAMPLE PREPARATION}

Samples were prepared for stable isotope analysis according to sample type and then processed using standard preservation methods (Hobson et al., 1997a, Jacob et al., 2005, Soreide et al., 2006, Carabel et al., 2006). Macroalgae samples were rinsed in de-ionised (DI) water, and prepared for analysis. Algae with calcium carbonate material was rinsed in $5 \% \mathrm{HCl}$ for 1 minute to dissolve the calcium carbonate and then rinsed again in DI water to remove the acid. Algae was then slowly dried between $40^{\circ}-45^{\circ} \mathrm{C}$ and ground in a pestle and mortar. Vascular plants were also prepared in the same manner as above.

Invertebrate, fish and marine mammal tissue samples were lipid extracted using a DIONEX 200 accelerated solvent extraction system (ASE). Samples were then transferred to $22 \mathrm{~mL}$ ASE cells and extracted three times with dichloromethane at $70^{\circ} \mathrm{C}$ and $1500 \mathrm{psi}$ for a static hold time of 5 minutes. Samples were then heated to $40^{\circ} \mathrm{C}$ in an oven overnight following lipid extraction, to evaporate any traces of solvent. Further drying was then undertaken at 50 degrees for 24 hours. Following 
lipid extraction, invertebrate, fish and marine mammal tissue was ground to a fine powder (Post et al., 2007b).

Marine invertebrate samples that had a high content of calcium carbonate (including species such as bryozoans) were acidified prior to analysis by covering the sample in few drops of $1 \mathrm{M} \mathrm{HCl}$ to separate tissue from the calcium carbonate. Acid was added until the sample stopped effervescing. Once the sample stopped bubbling it was rinsed with distilled water and then dried slowly and ground into a fine powder.

\subsubsection{STABLE ISOTOPE PROCESSING}

Following drying and grinding, samples were weighed into 1-2 g amounts and placed into tin boats and processed on a NA 1500 Fisons elemental analyzer linked to a ThermoFinnigan Delta plus mass spectrometer that provides the \% carbon and nitrogen and $\delta^{13} \mathrm{C}$ and $\delta^{15} \mathrm{~N}$ values.

In this thesis stable isotope data are expressed in parts per thousand (\%) using the following equation:

$$
\delta X=\left(\mathrm{R}_{\text {sample }} / \mathrm{R}_{\text {standard }}-1\right) \times 1000
$$

Where $\mathrm{X}={ }^{13} \mathrm{C}$ or ${ }^{15} \mathrm{~N}$, and $\mathrm{R}=$ ratio of heavy/light isotope content $\left({ }^{13} \mathrm{C} /{ }^{12} \mathrm{C}\right.$ or $\left.{ }^{15} \mathrm{~N} /{ }^{14} \mathrm{~N}\right)$.

\subsubsection{DATA ANALYSIS}

To determine if there was any effect of preparation of the samples on stable isotope values, two-tailed t-tests were used to compare the signatures of samples that had two different types of storage. Analysis was undertaken on algae samples that had been stored either fresh or in $70 \%$ ethanol. Two-tailed t-tests were undertaken on 
signatures from pressed fresh macroalgae and pressed fresh macroalgae that had been stored in $70 \%$ ethanol, to determine whether there was a significant difference between them. A two-tailed t-test was used to determine if there was any effect of lipid extraction on species' stable isotope signatures, by comparing samples that had been lipid extracted with those that had not been.

In order to provide an indication of the trophic relationships between species at the Bounty and Antipodes Islands, the means of all species’ $\delta^{13} \mathrm{C}$ and $\delta^{15} \mathrm{~N}$ signatures were plotted on an X-Y graph for each island. To describe the step-wise enrichment and provide an indication of each consumer's possible prey items, the mean $\delta^{13} \mathrm{C}$ of each species was graphed at each island and to describe the potential trophic position of each species, the means of each species' $\delta^{15} \mathrm{~N}$ were graphed for each island.

The trophic position of primary producers and consumers at each island were calculated using the model developed by Hobson and Welch (Hobson and Welch, 1992), which determines trophic level (TL) based on the $\delta^{15} \mathrm{~N}$ of the consumer (DM), the average $\delta^{15} \mathrm{~N}$ of the primary producers (PP - kelp and phytoplankton) and the $\delta^{15} \mathrm{~N}$ enrichment value per trophic level (E):

$\mathrm{TL}=1+(\mathrm{DM}-\mathrm{PP}) / \mathrm{E}$

The average kelp signature (PP) (4.1 at Antipodes Island and 3.65 at the Bounty Islands), was determined by pooling data from the dominant kelp species (Macrocystis pyrifera, Lessonia brevifolia, Marginariella parsonii) collected at each island. The phytoplankton signature used was the average of phytoplankton signatures from seawater samples collected every $2.5 \mathrm{~nm}$ within $12 \mathrm{~nm}$ of each island and then subsequently filtered (see Chapter 3). For this study a trophic enrichment factor (E) of 2.3 was used for all marine species (McCutchan et al., 2003). 
The resulting equations were:

Antipodes Island:

$$
\mathrm{TL}=1+(\mathrm{DM}-4.1) / 2.3
$$

Bounty Islands:

$$
\mathrm{TL}=1+(\mathrm{DM}-3.65) / 2.3
$$

A two-tailed t-test was used to determine if there was any significant difference in the $\delta^{13} \mathrm{C}$ and $\delta^{15} \mathrm{~N}$ values of species between sites at each island and to test for any difference between the Antipodes and Bounty Islands $\delta^{13} \mathrm{C}$ and $\delta^{15} \mathrm{~N}$ signatures.

All statistical analyses were undertaken using SPSS (SPSS, 2006). Where t-tests were conducted, data were first assessed for normality by plotting the data, then for homogeneity of variance using Levene's tests. The p-values subsequently used were based on the results of the Levene's tests. 
Table 2-1 List of samples collected from Antipodes Island including storage, treatment and stable isotope signatures.

\begin{tabular}{|c|c|c|c|c|c|c|}
\hline Sample & $n$ & $\begin{array}{l}\text { Average } \delta^{13} \mathrm{C} \\
\text { and SD }\end{array}$ & $\begin{array}{l}\text { Average } \delta^{15} \mathrm{~N} \\
\text { and SD }\end{array}$ & Tissue Type & Preservation & $\begin{array}{l}\text { Lipid } \\
\text { Extracted }\end{array}$ \\
\hline $\begin{array}{l}\text { Phytoplankton } \\
0-2 \mathrm{NM} \\
\text { 2-4.5NM } \\
4.5-7 \mathrm{NM} \\
\text { 7-9.5NM } \\
\text { 9.5-12NM }\end{array}$ & $\begin{array}{l}6 \\
3 \\
3 \\
3 \\
2\end{array}$ & $\begin{array}{l}-22.74 \pm 0.68 \\
-22.70 \pm 0.34 \\
-22.42 \pm 0.21 \\
-22.87 \pm 0.42 \\
-22.30 \pm 0.25\end{array}$ & $\begin{array}{r}-0.20 \pm 2.31 \\
-1.60 \pm 1.53 \\
-0.85 \pm 0.40 \\
1.25 \pm 0.39 \\
-0.69 \pm 0.95\end{array}$ & & $\begin{array}{l}\text { Fresh } \\
\text { Fresh } \\
\text { Fresh } \\
\text { Fresh } \\
\text { Fresh }\end{array}$ & $\begin{array}{l}\text { No } \\
\text { No } \\
\text { No } \\
\text { No } \\
\text { No }\end{array}$ \\
\hline $\begin{array}{l}\text { Zooplankton } \\
\text { Euphausiid }\end{array}$ & 1 & -21.29 & 5.58 & tails & Ethanol & Yes \\
\hline $\begin{array}{l}\text { Algae } \\
\text { Rhodophyta } \\
\text { Unidentified red } \\
\text { Callophyllis atrosanguinea } \\
\text { Coralline turf } \\
\text { Coralline encrusting } \\
\text { Phaeophyta } \\
\text { Cystophora } \\
\text { Unidentified brown } \\
\text { Marginariella parsonii } \\
\text { Xiphophora } \\
\text { Adenocystis } \\
\text { Durvillaea sp. Antipodes Island }\end{array}$ & $\begin{array}{l}5 \\
6 \\
1 \\
3 \\
\\
5 \\
3 \\
9 \\
4 \\
1 \\
?\end{array}$ & $\begin{array}{l}-31.65 \pm 1.00 \\
-34.59 \pm 0.39 \\
-8.27 \\
-8.73 \pm 1.63 \\
-12.70 \pm 0.42 \\
-10.42 \pm 1.79 \\
-27.83 \pm 3.96 \\
-13.60 \pm 0.01 \\
-9.17\end{array}$ & $\begin{array}{l}3.88 \pm 0.35 \\
2.36 \pm 0.28 \\
9.35 \\
2.87 \pm 0.35 \\
\\
10.72 \pm 3.27 \\
7.92 \pm 1.97 \\
4.18 \pm 0.70 \\
8.99 \pm 1.40 \\
11.70\end{array}$ & $\begin{array}{l}\text { Thallus } \\
\text { Thallus } \\
\text { Growth tips } \\
\text { Growth tips } \\
\text { Thallus } \\
\text { Thallus } \\
\text { Thallus } \\
\text { Thallus } \\
\text { Floats } \\
\text { Thallus }\end{array}$ & $\begin{array}{l}\text { Ethanol } \\
\text { Fresh/ Ethanol } \\
\text { Ethanol } \\
\text { Ethanol } \\
\text { Fresh } \\
\text { Fresh } \\
\text { Fresh/Ethanol } \\
\text { Ethanol } \\
\text { Ethanol } \\
\text { Ethanol }\end{array}$ & $\begin{array}{l}\text { No } \\
\text { No } \\
\text { No } \\
\text { No } \\
\text { No } \\
\text { No } \\
\text { No } \\
\text { No } \\
\text { No } \\
\text { No } \\
\text { No }\end{array}$ \\
\hline
\end{tabular}




\begin{tabular}{|c|c|c|c|c|c|c|}
\hline Sample & $n$ & $\begin{array}{l}\text { Average } \delta^{13} \mathrm{C} \\
\text { and SD }\end{array}$ & $\begin{array}{l}\text { Average } \delta^{15} \mathrm{~N} \\
\text { and SD }\end{array}$ & Tissue Type & Preservation & $\begin{array}{l}\text { Lipid } \\
\text { Extracted }\end{array}$ \\
\hline $\begin{array}{l}\text { Spatoglossum } \\
\text { Macrocystis pyrifera } \\
\text { Lessonia brevifolia } \\
\text { Haraldiophyllum crispatum } \\
\text { Chlorophyta } \\
\text { Codium } \\
\text { Chaetomorpha } \\
\text { Ulva } \\
\text { Zonaria }\end{array}$ & $\begin{array}{l}3 \\
3 \\
3 \\
6 \\
\\
3 \\
2 \\
1 \\
1\end{array}$ & $\begin{array}{l}-20.06 \pm 1.13 \\
-18.96 \pm 0.47 \\
-16.15 \pm 0.44 \\
-34.02 \pm 0.47 \\
\\
-14.07 \pm 3.38 \\
-17.35 \pm 0.16 \\
-13.91 \\
-20.96\end{array}$ & $\begin{array}{l}3.23 \pm 0.34 \\
4.08 \pm 0.10 \\
4.09 \pm 0.54 \\
1.46 \pm 0.20 \\
\\
8.17 \pm 1.37 \\
4.14 \pm 0.06 \\
9.36 \\
3.81\end{array}$ & $\begin{array}{l}\text { Thallus } \\
\text { thallus } \\
\text { Thallus } \\
\text { Thallus } \\
\text { Thallus } \\
\text { Tips } \\
\text { Tips of blade } \\
\text { tips }\end{array}$ & $\begin{array}{l}\text { Ethanol } \\
\text { Ethanol } \\
\text { Ethanol } \\
\text { Fresh/Ethanol } \\
\text { Ethanol } \\
\text { Ethanol } \\
\text { Ethanol } \\
\text { Ethanol }\end{array}$ & $\begin{array}{l}\text { No } \\
\text { No } \\
\text { No } \\
\text { No } \\
\text { No } \\
\text { No } \\
\text { No } \\
\text { No } \\
\text { No }\end{array}$ \\
\hline $\begin{array}{l}\text { Porifera } \\
\text { Darwinella gardineri } \\
\text { Latrunculia brevis }\end{array}$ & $\begin{array}{l}1 \\
1\end{array}$ & $\begin{array}{l}-21.60 \\
-19.11\end{array}$ & $\begin{array}{l}2.35 \\
4.20\end{array}$ & $\begin{array}{l}\text { Growth edges } \\
\text { Growth edges }\end{array}$ & $\begin{array}{l}\text { Ethanol } \\
\text { Ethanol }\end{array}$ & $\begin{array}{l}\text { Yes } \\
\text { Yes } \\
\text { yes }\end{array}$ \\
\hline $\begin{array}{l}\text { Cnidaria } \\
\text { Hydroid } \\
\text { Anenomes } \\
\end{array}$ & $\begin{array}{l}2 \\
3\end{array}$ & $\begin{array}{l}-21.72 \pm 0.16 \\
-20.60 \pm 0.07\end{array}$ & $\begin{array}{l}0.50 \pm 0.67 \\
5.11 \pm 1.05\end{array}$ & $\begin{array}{l}\text { Colony tips } \\
\text { Wall muscle }\end{array}$ & $\begin{array}{l}\text { Ethanol } \\
\text { Ethanol }\end{array}$ & $\begin{array}{l}\text { Yes } \\
\text { Yes }\end{array}$ \\
\hline $\begin{array}{l}\text { Crustacea } \\
\text { Leptomithrax australis } \\
\text { Tubeworms } \\
\text { Balanus }\end{array}$ & $\begin{array}{l}1 \\
3 \\
1\end{array}$ & $\begin{array}{l}-18.48 \\
-20.75 \pm 0.46 \\
-21.72\end{array}$ & $\begin{array}{l}10.29 \\
5.46 \pm 0.19 \\
5.14\end{array}$ & Leg muscle & $\begin{array}{l}\text { Ethanol } \\
\text { Ethanol } \\
\text { Ethanol }\end{array}$ & $\begin{array}{l}\text { Yes } \\
\text { Yes } \\
\text { Yes }\end{array}$ \\
\hline $\begin{array}{l}\text { Mollusca } \\
\text { Eudoxochiton nobilis } \\
\text { Aulacomya maoriana }\end{array}$ & $\begin{array}{l}4 \\
3\end{array}$ & $\begin{array}{l}-22.71 \pm 5.13 \\
-21.43 \pm 0.46\end{array}$ & $\begin{array}{l}6.09 \pm 2.33 \\
1.25 \pm 0.79\end{array}$ & $\begin{array}{l}\text { Foot muscle } \\
\text { Foot muscle }\end{array}$ & $\begin{array}{l}\text { Ethanol } \\
\text { Ethanol }\end{array}$ & $\begin{array}{l}\text { Yes } \\
\text { Yes }\end{array}$ \\
\hline
\end{tabular}




\begin{tabular}{|c|c|c|c|c|c|c|}
\hline Sample & $n$ & $\begin{array}{l}\text { Average } \delta^{13} \mathrm{C} \\
\text { and SD }\end{array}$ & $\begin{array}{l}\text { Average } \delta^{15} \mathrm{~N} \\
\text { and SD }\end{array}$ & Tissue Type & Preservation & $\begin{array}{l}\text { Lipid } \\
\text { Extracted }\end{array}$ \\
\hline $\begin{array}{l}\text { Buccinulum pertinax } \\
\text { Cellana strigilis } \\
\text { Haliotis virginea huttoni } \\
\text { Calliostoma eminens } \\
\text { Fusitriton laudandus }\end{array}$ & $\begin{array}{l}3 \\
4 \\
3 \\
3 \\
3\end{array}$ & $\begin{array}{l}-20.60 \pm 0.08 \\
-12.72 \pm 2.48 \\
-23.92 \pm 0.69 \\
-19.31 \pm 0.32 \\
-19.44 \pm 0.39\end{array}$ & $\begin{array}{l}6.37 \pm 0.30 \\
10.89 \pm 0.76 \\
5.60 \pm 0.34 \\
7.53 \pm 0.67 \\
6.41 \pm 0.69\end{array}$ & $\begin{array}{l}\text { Foot muscle } \\
\text { Foot muscle } \\
\text { Foot muscle } \\
\text { Foot muscle } \\
\text { Foot muscle }\end{array}$ & $\begin{array}{l}\text { Ethanol } \\
\text { Ethanol } \\
\text { Ethanol } \\
\text { Ethanol } \\
\text { Ethanol }\end{array}$ & $\begin{array}{l}\text { Yes } \\
\text { Yes } \\
\text { Yes } \\
\text { Yes } \\
\text { Yes }\end{array}$ \\
\hline $\begin{array}{l}\text { Bryozoa } \\
\text { Bryozoans }\end{array}$ & 4 & $-4.25 \pm 1.49$ & $0.81 \pm 0.90$ & Colony tips & Ethanol & yes \\
\hline $\begin{array}{l}\text { Echinodermata } \\
\text { Henricia sp } \\
\text { Paranepanthia aucklandensis } \\
\text { Ophiomyxa brevirima } \\
\text { Squamocnus brevidentis }\end{array}$ & $\begin{array}{l}3 \\
4 \\
2 \\
2\end{array}$ & $\begin{array}{l}-20.31 \pm 0.39 \\
-17.43 \pm 1.29 \\
-4.31 \pm 1.20 \\
-17.65 \pm 1.41\end{array}$ & $\begin{array}{l}3.86 \pm 0.94 \\
9.92 \pm 1.17 \\
9.10 \pm 0.09 \\
5.90 \pm 0.78\end{array}$ & $\begin{array}{l}\text { Tentacles } \\
\text { Tentacles } \\
\text { Whole arm } \\
\text { Tentacles }\end{array}$ & $\begin{array}{l}\text { Ethanol } \\
\text { Ethanol } \\
\text { Ethanol } \\
\text { Ethanol }\end{array}$ & $\begin{array}{l}\text { Yes } \\
\text { Yes } \\
\text { Yes } \\
\text { Yes }\end{array}$ \\
\hline $\begin{array}{l}\text { Chordata } \\
\text { Tunicata } \\
\text { Salp } \\
\text { Fish } \\
\text { Bovichtus variegatis } \\
\text { Marine Birds } \\
\text { Diomedea antipodensis feather } \\
\text { Diomedea antipodensis skin } 1 \\
\text { Diomedea antipodensis skin2 } \\
\text { Eudyptes sclateri feather } \\
\text { Eudyptes sclateri skin } \\
\text { Macronectes halli } \\
\text { Pterodroma sp }\end{array}$ & $\begin{array}{l}2 \\
1 \\
4 \\
3 \\
3 \\
3 \\
3\end{array}$ & $\begin{array}{l}-22.08 \\
-19.49 \\
-20.72 \pm 0.16 \\
-18.21 \\
-19.02 \pm 0.65 \\
-20.44 \pm 1.06 \\
-19.39 \pm 0.66 \\
-20.75 \pm 1.26 \\
-21.84 \pm 0.96\end{array}$ & $\begin{array}{l}-1.97 \\
7.49 \\
13.12 \pm 0.49 \\
16.33 \\
15.36 \pm 1.40 \\
8.35 \pm 0.80 \\
7.83 \pm 0.96 \\
13.78 \pm 1.23 \\
10.86 \pm 3.34\end{array}$ & $\begin{array}{l}\text { tunic section } \\
\text { Muscle } \\
\text { Feather } \\
\text { Skin } \\
\text { Skin } \\
\text { Feather } \\
\text { Skin } \\
\text { Feather } \\
\text { Feather }\end{array}$ & $\begin{array}{l}\text { Ethanol } \\
\text { Ethanol } \\
\text { Fresh }\end{array}$ & $\begin{array}{l}\text { Yes } \\
\text { Yes } \\
\text { No } \\
\text { Yes } \\
\text { Yes } \\
\text { No } \\
\text { Yes } \\
\text { No } \\
\text { No }\end{array}$ \\
\hline
\end{tabular}




\begin{tabular}{|c|c|c|c|c|c|c|}
\hline Sample & $n$ & $\begin{array}{l}\text { Average } \delta^{13} \mathrm{C} \\
\text { and SD }\end{array}$ & $\begin{array}{l}\text { Average } \delta^{15} \mathrm{~N} \\
\text { and SD }\end{array}$ & Tissue Type & Preservation & $\begin{array}{l}\text { Lipid } \\
\text { Extracted }\end{array}$ \\
\hline $\begin{array}{l}\text { Pterodroma lessonii } \\
\text { Procellaria cinerea } \\
\text { Pelecanoides urinatrix }\end{array}$ & $\begin{array}{l}3 \\
6 \\
3\end{array}$ & $\begin{array}{l}-20.82 \pm 0.60 \\
-20.13 \pm 0.93 \\
-21.21 \pm 1.37\end{array}$ & $\begin{array}{l}11.33 \pm 0.15 \\
11.24 \pm 1.53 \\
8.98 \pm 0.30\end{array}$ & $\begin{array}{l}\text { Feather } \\
\text { Feather } \\
\text { Feather }\end{array}$ & $\begin{array}{l}\text { Fresh } \\
\text { Fresh } \\
\text { Fresh }\end{array}$ & $\begin{array}{l}\text { No } \\
\text { No } \\
\text { No }\end{array}$ \\
\hline $\begin{array}{l}\text { Terrestrial birds } \\
\text { Cyanoramphus unicolor } \\
\text { Cyanoramphus novaezelandiae } \\
\text { hochstetteri } \\
\text { Snipe }\end{array}$ & $\begin{array}{l}3 \\
2 \\
2\end{array}$ & $\begin{array}{l}-23.23 \pm 1.89 \\
-24.01 \pm 1.89 \\
-22.45 \pm 0.38\end{array}$ & $\begin{array}{l}16.18 \pm 0.52 \\
10.12 \pm 0.19 \\
14.11 \pm 1.69\end{array}$ & $\begin{array}{l}\text { Feather } \\
\text { Feather } \\
\text { Feather }\end{array}$ & $\begin{array}{l}\text { Fresh } \\
\text { Fresh } \\
\text { Fresh }\end{array}$ & $\begin{array}{l}\text { No } \\
\text { No } \\
\text { no }\end{array}$ \\
\hline $\begin{array}{l}\text { Terrestrial plants } \\
\text { Moss } \\
\text { Grass } \\
\text { Fern } \\
\text { Lichen } \\
\text { Bush/shrub } \\
\text { Plant from penguin colony }\end{array}$ & $\begin{array}{l}4 \\
3 \\
4 \\
4 \\
2 \\
2\end{array}$ & $\begin{array}{l}-18.93 \pm 4.19 \\
-24.37 \pm 2.35 \\
-26.66 \pm 0.37 \\
-18.36 \pm 1.43 \\
-24.82 \pm 0.47 \\
-24.54 \pm 0.47\end{array}$ & $\begin{array}{l}9.76 \pm 1.69 \\
17.26 \pm 4.57 \\
10.09 \pm 0.95 \\
5.73 \pm 5.18 \\
11.14 \pm 3.13 \\
22.51 \pm 6.27\end{array}$ & $\begin{array}{l}\text { Tips } \\
\text { Blade } \\
\text { Leaf } \\
\text { Tips } \\
\text { Leaf } \\
\text { Leaf }\end{array}$ & $\begin{array}{l}\text { Fresh } \\
\text { Fresh } \\
\text { Fresh } \\
\text { Fresh } \\
\text { Fresh } \\
\text { Fresh }\end{array}$ & $\begin{array}{l}\text { No } \\
\text { No } \\
\text { No } \\
\text { No } \\
\text { No } \\
\text { No }\end{array}$ \\
\hline $\begin{array}{l}\text { Terrestrial Invertebrates } \\
\text { worms } \\
\text { Flies } \\
\text { Fleas } \\
\text { Ticks }\end{array}$ & $\begin{array}{l}1 \\
3 \\
2 \\
1\end{array}$ & $\begin{array}{l}-14.07 \\
-21.86 \pm 0.37 \\
-20.86 \pm 0.44 \\
-17.39\end{array}$ & $\begin{array}{l}12.46 \\
25.27 \pm 1.80 \\
12.93 \pm 0.15 \\
14.47\end{array}$ & $\begin{array}{l}\text { Whole animal } \\
\text { Whole animal } \\
\text { Whole animal } \\
\text { Whole animal }\end{array}$ & $\begin{array}{l}\text { Ethanol } \\
\text { Ethanol } \\
\text { Ethanol } \\
\text { Ethanol }\end{array}$ & $\begin{array}{l}\text { Yes } \\
\text { Yes } \\
\text { Yes } \\
\text { Yes }\end{array}$ \\
\hline $\begin{array}{l}\text { Terrestrial mammal } \\
\text { Mus musculus }\end{array}$ & 3 & $-18.93 \pm 0.65$ & $9.76 \pm 1.69$ & Tip of tail & Ethanol & Yes \\
\hline
\end{tabular}




\begin{tabular}{|c|c|c|c|c|c|c|}
\hline Sample & $n$ & $\begin{array}{l}\text { Average } \delta^{13} \mathrm{C} \\
\text { and SD }\end{array}$ & $\begin{array}{l}\text { Average } \delta^{15} \mathrm{~N} \\
\text { and SD }\end{array}$ & Tissue Type & Preservation & $\begin{array}{l}\text { Lipid } \\
\text { Extracted }\end{array}$ \\
\hline $\begin{array}{l}\text { Marine mammals } \\
\text { Arctocephalus forsteri } \\
\text { Mirounga leonina }\end{array}$ & $\begin{array}{l}4 \\
4\end{array}$ & $\begin{array}{l}-17.74 \pm 0.20 \\
-17.78 \pm 1.33\end{array}$ & $\begin{array}{l}12.20 \pm 0.79 \\
15.02 \pm 0.66\end{array}$ & $\begin{array}{l}\text { Skin } \\
\text { Skin }\end{array}$ & $\begin{array}{l}\text { Ethanol } \\
\text { Ethanol }\end{array}$ & $\begin{array}{l}\text { Yes } \\
\text { Yes }\end{array}$ \\
\hline $\begin{array}{l}\text { Guano and scat } \\
\text { Anthus steindachneri } \\
\text { Stercorarius skua lonnbergi } \\
\text { Eudyptes sclateri } \\
\text { Arctocephalus forsteri }\end{array}$ & $\begin{array}{l}1 \\
1 \\
6 \\
4(N) \\
3(C)\end{array}$ & $\begin{array}{l}-22.29 \\
-9.11 \\
-23.88 \pm 0.35 \\
-23.39 \pm 0.65\end{array}$ & $\begin{array}{l}26.31 \\
16.62 \\
7.19 \pm 3.18 \\
16.76 \pm 3.93\end{array}$ & $\begin{array}{l}\text { Guano } \\
\text { Guano } \\
\text { Guano } \\
\text { Guano }\end{array}$ & $\begin{array}{l}\text { Fresh } \\
\text { Fresh } \\
\text { Fresh } \\
\text { Fresh }\end{array}$ & $\begin{array}{l}\text { No } \\
\text { No } \\
\text { No } \\
\text { No }\end{array}$ \\
\hline
\end{tabular}


Table 2-2 List of samples collected from the Bounty Islands including storage, preparation and stable isotope signatures.

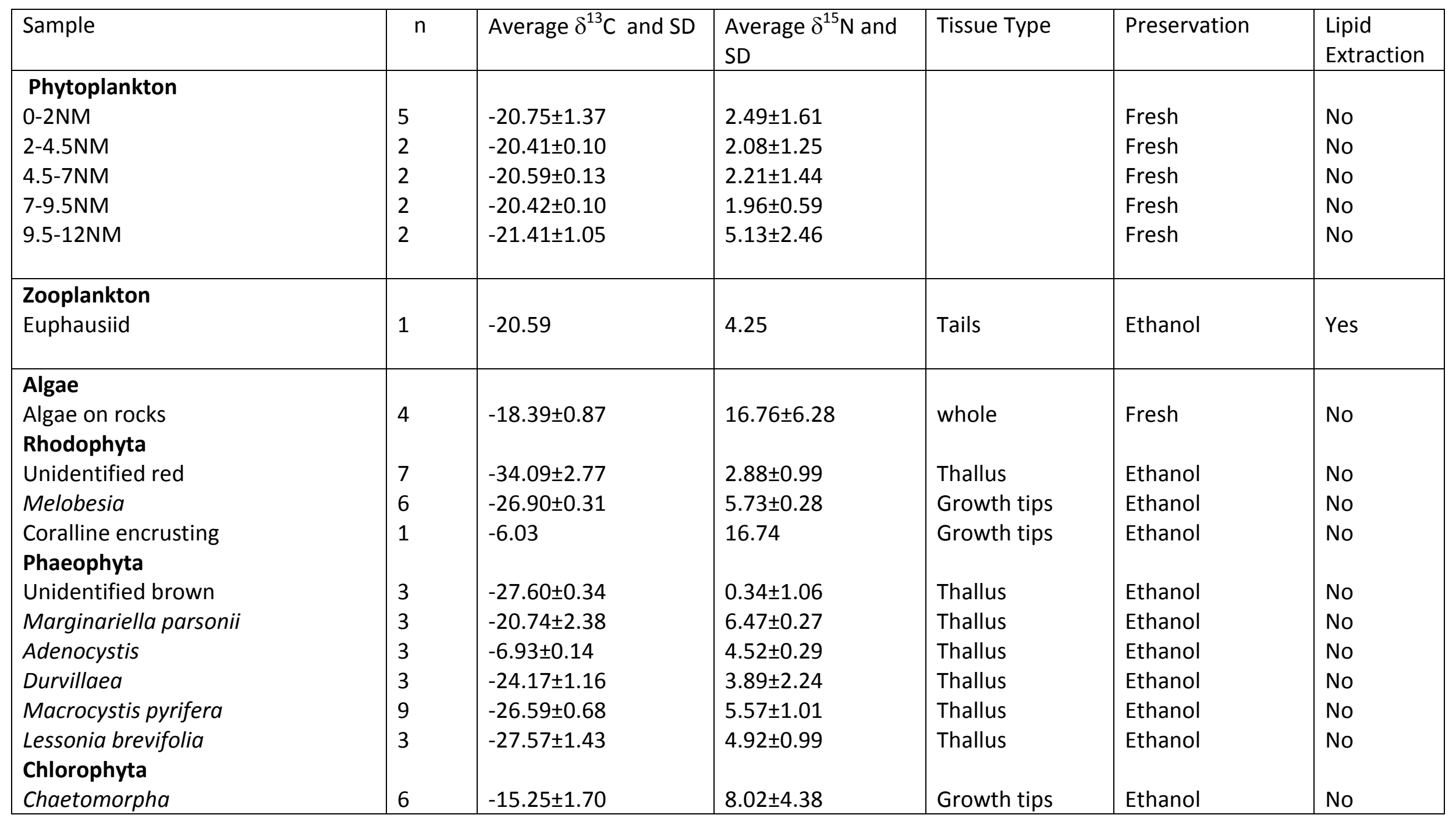




\begin{tabular}{|c|c|c|c|c|c|c|}
\hline Unidentified & 3 & $-22.32 \pm 2.94$ & $3.98 \pm 4.73$ & Thallus & Fresh & No \\
\hline $\begin{array}{l}\text { Cnidaria } \\
\text { Anenomes }\end{array}$ & 3 & $-18.38 \pm 0.55$ & $7.63 \pm 0.56$ & Our wall & Ethanol & Yes \\
\hline $\begin{array}{l}\text { Crustacea } \\
\text { Leptomithrax australis } \\
\text { Balanus } \\
\text { Shrimps } \\
\text { Talorchestia }\end{array}$ & $\begin{array}{l}3 \\
3 \\
1 \\
4 \\
\end{array}$ & $\begin{array}{l}-18.84 \pm 0.35 \\
-19.02 \pm 0.36 \\
-18.62 \\
-18.48 \pm 1.49\end{array}$ & $\begin{array}{l}9.81 \pm 1.51 \\
7.14 \pm 0.85 \\
8.71 \\
21.40 \pm 3.27\end{array}$ & $\begin{array}{l}\text { leg muscle } \\
\text { Foot muscle } \\
\text { Tail } \\
\text { Tail }\end{array}$ & $\begin{array}{l}\text { Ethanol } \\
\text { Ethanol } \\
\text { Ethanol } \\
\text { Ethanol }\end{array}$ & $\begin{array}{l}\text { Yes } \\
\text { Yes } \\
\text { Yes } \\
\text { Yes }\end{array}$ \\
\hline $\begin{array}{l}\text { Bryozoa } \\
\text { Bryozoan }\end{array}$ & 10 & $-13.32 \pm 6.87$ & $2.84 \pm 0.93$ & Colony tips & Ethanol & Yes \\
\hline
\end{tabular}




\begin{tabular}{|c|c|c|c|c|c|c|}
\hline Orthoscuticella ventricosa & 3 & $-6.38 \pm 0.57$ & $1.58 \pm 1.36$ & Colony tips & Ethanol & Yes \\
\hline $\begin{array}{l}\text { Echinodermata } \\
\text { Henricia } s p \\
\text { Starfish }\end{array}$ & $\begin{array}{l}3 \\
6\end{array}$ & $\begin{array}{l}-16.74 \pm 1.25 \\
-16.61 \pm 0.48\end{array}$ & $\begin{array}{l}7.30 \pm 0.41 \\
9.13 \pm 0.37\end{array}$ & $\begin{array}{l}\text { suckers } \\
\text { Tentacles }\end{array}$ & $\begin{array}{l}\text { Ethanol } \\
\text { Ethanol }\end{array}$ & $\begin{array}{l}\text { Yes } \\
\text { Yes }\end{array}$ \\
\hline $\begin{array}{l}\text { Chordata } \\
\text { Marine Birds } \\
\text { Diomedea cauta salvini - chick } \\
\text { Diomedea cauta salvini - NB Adult } \\
\text { Diomedea cauta salvini - Dead } \\
\text { Eudyptes sclateri }\end{array}$ & $\begin{array}{l}3 \\
3 \\
3 \\
4\end{array}$ & $\begin{array}{l}-17.73 \pm 0.69 \\
-15.96 \pm 2.28 \\
-19.86 \pm 2.32 \\
-20.46 \pm 0.92\end{array}$ & $\begin{array}{l}14.54 \pm 1.23 \\
17.91 \pm 5.28 \\
14.59 \pm 0.63 \\
9.65 \pm 0.56\end{array}$ & $\begin{array}{l}\text { Feather } \\
\text { Feather } \\
\text { Feather } \\
\text { Feather }\end{array}$ & $\begin{array}{l}\text { Fresh } \\
\text { Fresh } \\
\text { Fresh } \\
\text { Fresh }\end{array}$ & $\begin{array}{l}\text { No } \\
\text { No } \\
\text { No } \\
\text { No }\end{array}$ \\
\hline $\begin{array}{l}\text { Terrestrial Invertebrates } \\
\text { Mosquitoes } \\
\text { Pacificana }\end{array}$ & $\begin{array}{l}3 \\
3\end{array}$ & $\begin{array}{l}-18.78 \pm 0.50 \\
-18.11 \pm 0.14\end{array}$ & $\begin{array}{l}22.84 \pm 2.09 \\
28.10 \pm 1.40\end{array}$ & $\begin{array}{l}\text { Whole } \\
\text { Whole }\end{array}$ & $\begin{array}{l}\text { Ethanol } \\
\text { Ethanol }\end{array}$ & $\begin{array}{l}\text { Yes } \\
\text { Yes }\end{array}$ \\
\hline $\begin{array}{l}\text { Guano and scat } \\
\text { Eudyptes sclateri } \\
\text { Arctocephalus forsteri }\end{array}$ & $\begin{array}{l}4 \\
5\end{array}$ & $\begin{array}{l}-23.05 \pm 1.04 \\
-22.91 \pm 1.85\end{array}$ & $\begin{array}{l}15.13 \pm 7.10 \\
15.13 \pm 1.51\end{array}$ & $\begin{array}{l}\text { Guano } \\
\text { Scat }\end{array}$ & $\begin{array}{l}\text { Fresh } \\
\text { Fresh }\end{array}$ & $\begin{array}{l}\text { No } \\
\text { No }\end{array}$ \\
\hline
\end{tabular}


Table 2-3 Physical habitats recorded at Antipodes and Bounty Islands during March 2009.

\begin{tabular}{|c|c|c|}
\hline Island Group & Physical habitat & Locations habitat recorded \\
\hline \multirow{8}{*}{$\begin{array}{l}\text { Antipodes } \\
\text { Island }\end{array}$} & Cobble beach & Hut Cove \\
\hline & Boulder beach & $\begin{array}{l}\text { Ringdove Bay, Anchorage Bay, } \\
\text { Mirounga Bay }\end{array}$ \\
\hline & Rocky platform & Anchorage Bay \\
\hline & Shallow rocky reef & $\begin{array}{l}\text { Ringdove Bay, Heck's Head, } \\
\text { Windward Island, Stack Bay, Orde } \\
\text { Lees Islet, Orde Lees penguin colony, } \\
\text { North Bollon's Island, Leeward } \\
\text { Island, Anchorage Bay. }\end{array}$ \\
\hline & Deep rocky reef & $\begin{array}{l}\text { Orde Lees Islet, Orde Lees penguin } \\
\text { colony }\end{array}$ \\
\hline & Deep sand & Alert Bay \\
\hline & $\begin{array}{l}\text { Dense grasslands, } \\
\text { ferns, peat bog - } \\
\text { Terrestrial }\end{array}$ & Hut Point \\
\hline & $\begin{array}{l}\text { Dense grasslands, } \\
\text { ferns, Coprosmas, } \\
\text { herbs, peat bog - } \\
\text { Terrestrial }\end{array}$ & Plateau \\
\hline \multirow[t]{7}{*}{ Bounty Islands } & Rocky platform & Depot Island \\
\hline & Shallow rocky reef & $\begin{array}{l}\text { Tunnel Island, Lion Island, South } \\
\text { Ruatara Island, east Proclamation } \\
\text { Island, southeast Proclamation Island, } \\
\text { Funnel Island west, Molly Cap, north } \\
\text { Proclamation Island, Skua / Spider } \\
\text { gut, Funnel Island east, south Tunnel } \\
\text { Island, west Depot Island, northwest } \\
\text { Ruatara Island. }\end{array}$ \\
\hline & $\begin{array}{l}\text { Shallow boulder } \\
\text { field }\end{array}$ & South Tunnel Island \\
\hline & Shallow gravel field & Tunnel Island \\
\hline & Deep rocky reef & $\begin{array}{l}\text { Tunnel Island, South Tunnel Island, } \\
\text { Lion Island, Molly Cap, east } \\
\text { Proclamation Island, northwest } \\
\text { Ruatara Island, Skua / Spider gut. }\end{array}$ \\
\hline & Deep gravel field & Tunnel Island. \\
\hline & $\begin{array}{l}\text { Granite rock, guano, } \\
\text { algae -Terrestrial }\end{array}$ & Depot Island \\
\hline
\end{tabular}


Figure 2-1 Map showing the location of the Bounty and Antipodes Islands.

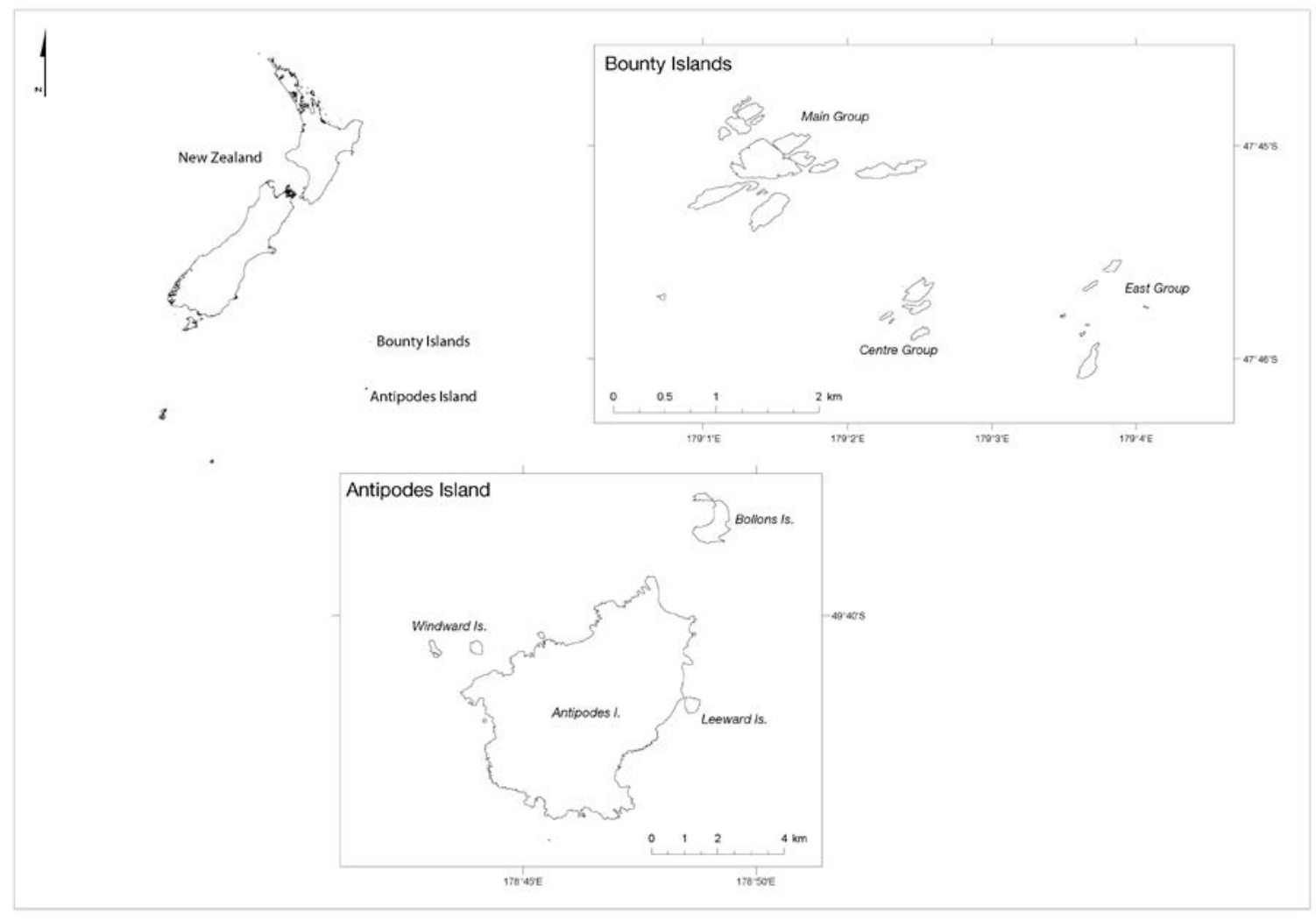


Figure 2-2 Maps showing the sites where samples were collected for stable isotope analysis at the Bounty (a) and Antipodes Islands (b).

(a)

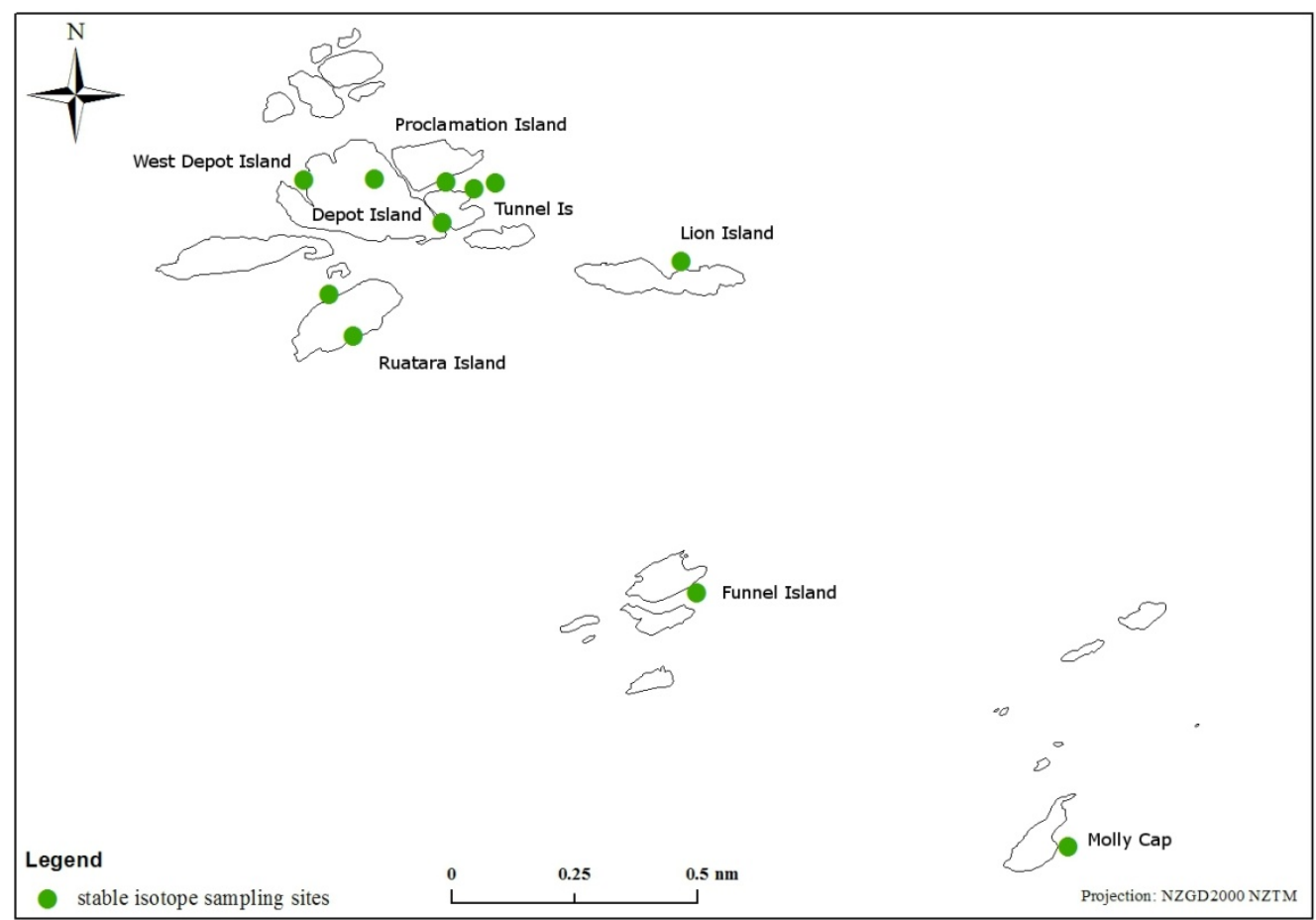

(b)

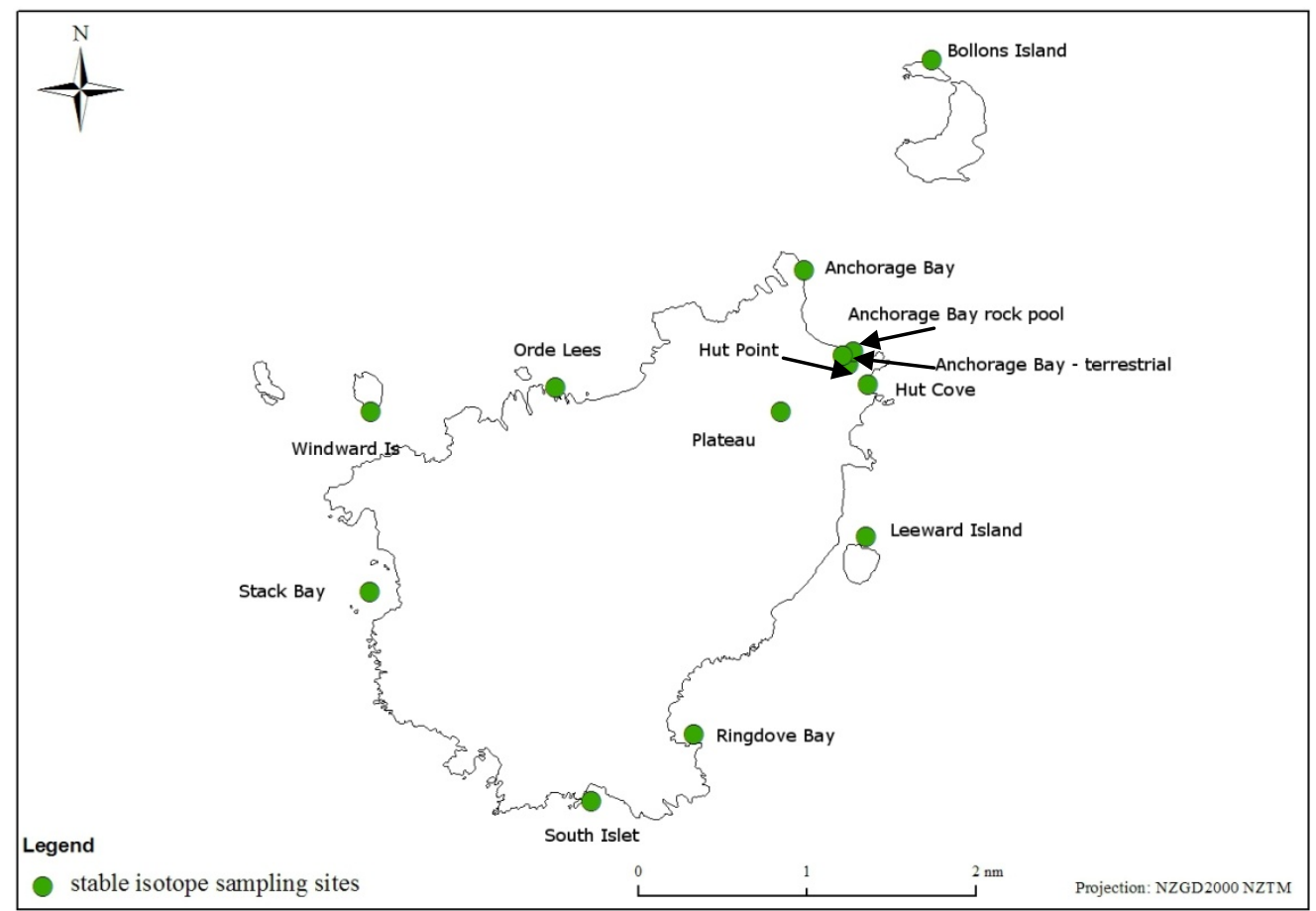


Figure 2-3 Photograph of water-filtering apparatus to collect phytoplankton.

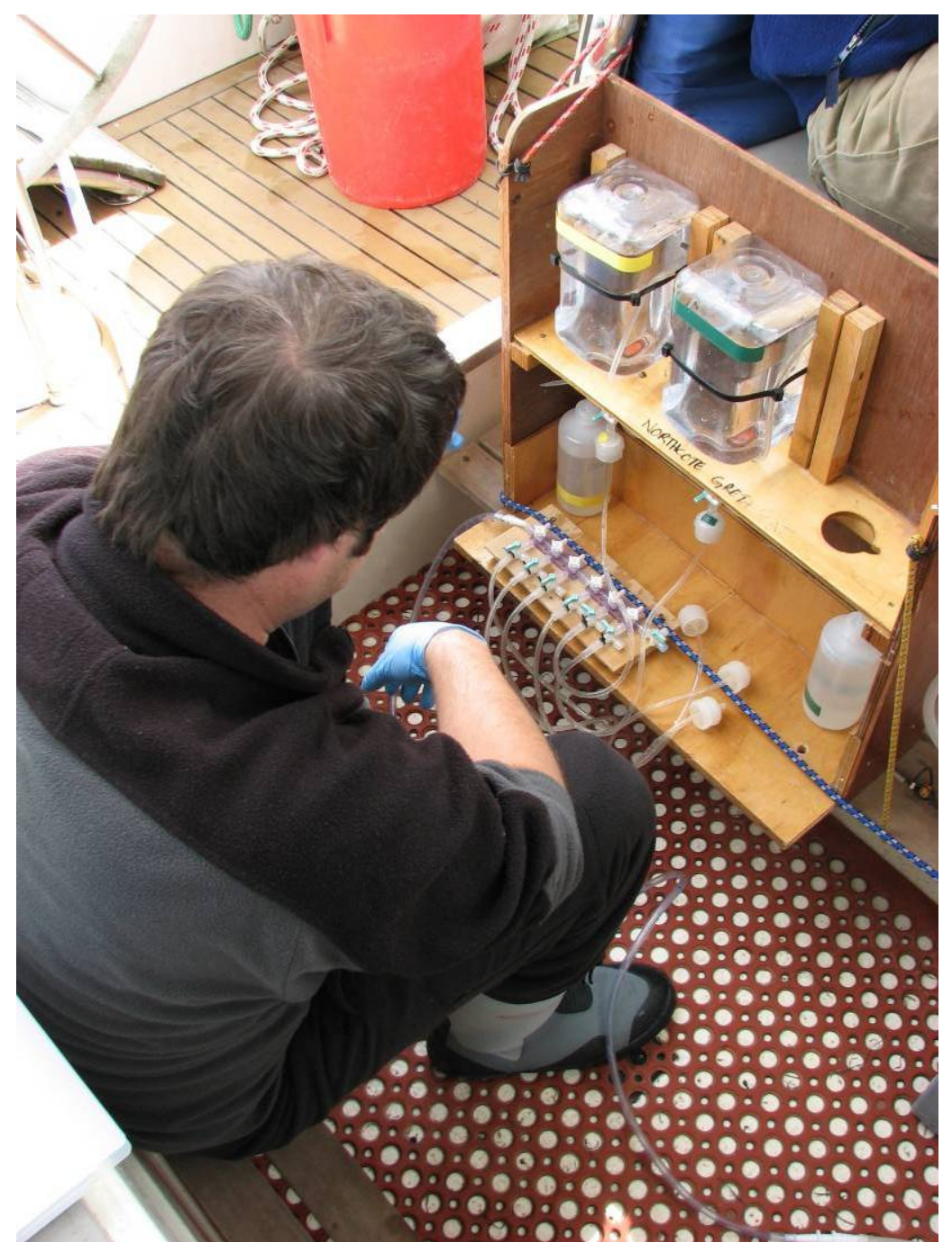




\subsection{RESULTS}

\subsubsection{EFFECT OF SAMPLE PREPARATION}

Fresh samples of macroalgae and those of the same species preserved in 70\% ethanol in the field, did not show a significant difference in their stable isotope signatures (two-tailed $t$-tests, $\mathrm{p}>0.05$ ). Samples that had been lipid extracted did not show any significant difference to those that had not been lipid-extracted (two-tailed $t$-tests, $\mathrm{p}>0.05$ ).

\subsubsection{ISOTOPIC COMPOSITION OF ANTIPODES ISLAND AND BOUNTY ISLANDS}

There was considerable variation in the mean carbon stable isotope signatures across the food web at Antipodes Island (Figure 2-4, Figure 2-5). There was no noticeable stepwise enrichment evident in the mean values of carbon at Antipodes Island and the carbon signatures did not display any apparent relationship to trophic position.

The distribution of carbon stable isotope ratios ranged from $4.25 \%$ \pm 1.49 (mean \pm SE) for a bryozoan species, to $-34.59 \% \pm 0.39$ for Callophyllis atrosanguinea (a red algae). There was also considerable variation in the mean values of nitrogen for Antipodes Island (Figure 2-4, Figure 2-6). However, in contrast to carbon, there was a more distinct trend in the nitrogen values of species relative to their trophic position (Figure 2-6, Figure 2-7). The single salp collected had an extremely low nitrogen value of -1.97 and the highest recorded value was for the flies captured within the penguin colonies $(25.27 \%$ o \pm 1.80$)$.

The mean carbon and nitrogen values for Bounty Islands species ranged widely (Figure 2-8, Figure 2-9, Figure 2-10). Carbon values ranged from the most depleted Callophyllis astrosanguinea $(-34.59 \% \pm \pm 2.77)$ to the highest value of 6.03 for an encrusting coralline algae (Figure 2-8, Figure 2-9). As with 
Antipodes Island, there was no noticeable stepwise enrichment in mean carbon values at the Bounty Islands (Figure 2-9). Similar to Antipodes Island, there was a pattern for increasing nitrogen values with trophic levels (Figure 2-10, Figure 2-11). The highest nitrogen stable isotope signature was $28.10 \%$ for Pacificana (a terrestrial spider). The lowest nitrogen signature was $0.95 \%$ for an unidentified brown algae.

\section{Phytoplankton}

There was little difference in the range of mean carbon signatures of phytoplankton at the 2.5NM sampling stations within 12NM of the Antipodes Island, with a .57 \%o difference between the highest and lowest values. Mean nitrogen values ranged from $-1.60 \%$ o \pm 1.53 to $1.25 \%$ o \pm 0.39 . Similarly, there was little variation in mean carbon values of phytoplankton at the 2.5NM sampling stations within 12 nautical miles of the Bounty Islands with a difference of .33\%o between the highest and lowest values. Mean nitrogen values ranged between the lowest value of $1.96 \%$ o \pm 0.59 and the highest of $5.13 \%$ o \pm 1.05 .

\section{Macroalgae}

The mean carbon signature of macroalgae at Antipodes Island varied considerably and the most depleted mean carbon value was found for the rhodophyte Callophyllis atrosanguinea $(-34.59 \%$ \pm 0.39$)$ and least depleted for a single sample of the brown alga Adenocystis (-9.17\%o). A wide range of mean nitrogen values was found for macroalgae, with the phaeophyte Haraldiophyllum crispatum being the least enriched $(1.46 \% \pm 0.20)$, while the most enriched of the macroalgae was Cystophora (10.72\% \pm 3.37$)$.Amongst the mean carbon macroalgae signatures at the Bounty Islands, considerable variability was found across all species particularly in the rhodophyta. Species of rhodophyta were found to have both the lowest and highest carbon values of all the macroalgae. Macroalgae showed considerable variability in their mean nitrogen values - the lowest nitrogen ratio of $0.95 \%$ was found for an unidentified brown algae and the most enriched value of 16.74\%o for encrusting coralline algae (also an unidentified species). 


\section{Zooplankton}

Euphausiids were the only zooplankton collected at Antipodes Island; several individuals (of the same unidentified species) were combined and provided a carbon value of $-21.29 \%$ and a nitrogen value of 5.58\%o. Only one zooplankton sample was collected from the Bounty Islands and had a carbon value of $20.59 \%$ and a nitrogen value of $4.25 \%$.

\section{Suspension-feeding Invertebrates}

Amongst the suspension feeders at Antipodes Island, there was considerable variability in the carbon values, with the most depleted being a single salp ($22.08 \%$ o) and the highest value a pooled collection of bryozoans $(-4.25 \%$ o \pm 1.49$)$. However, the majority of suspension feeders showed little variation in carbon signature (within 1\%o). Hydroids had the lowest mean nitrogen value of $0.50 \%$ o \pm 0.67 and the highest mean nitrogen value was found for tubeworms (5.46 \%o \pm 0.19 ). The suspension feeders showed low variability in mean carbon values at the Bounty Islands. The most depleted suspension feeder was the sponge Callyspongia sp.nov 12, which had a carbon value of $-19.67 \%$ o \pm 0.57 and the highest and the most variable carbon value was found in a pooled sample of bryozoans (-13.32 \pm 6.87$)$. Filter feeders at the Bounty Islands did not show a large range in their nitrogen values; the lowest value of $2.93 \%$ o \pm 0.12 was found for an unidentified sponge and the highest mean nitrogen value of $7.63 \%$ \% \pm 0.56 for anemones (one unidentified species).

\section{Grazers}

Grazers showed considerable variation in carbon signatures at Antipodes Island, with paua Haliotis virginea huttoni being the most carbon depleted (-23.92\% \pm 0.69 ) and limpet Cellana strigilis the least depleted (12.72\% \pm 2.48$)$. The lowest mean nitrogen value was found for Haliotis virginea huttoni $(5.60 \% \pm 0.34)$ and the most enriched mean nitrogen value for Cellana strigilis (10.72\% \pm 0.76 ). Grazers at the Bounty Islands displayed a considerable range in their carbon 
values with Haliotis virginea huttoni being the most depleted (-22.56\% \pm 0.33 ) and the least depleted the limpet Kerguelenella lateralis $(-14.36 \% \pm 0.82)$. Of note was the high variation between Cellana strigilis samples $(-16.81 \%$ \pm 4.86$)$. Mean nitrogen ranged between the lowest for Haliotis virginea huttoni (6.27 \pm 0.76$)$ and the highest and most variable for the chiton Onithochiton neglectus (11.91土5.33).

\section{Opportunistic feeders / Omnivores}

Amongst the opportunistic feeders/omnivores at Antipodes Island the whelk Buccinulum pertinax was the most depleted in its carbon value $(-20.60 \% \pm 0.08)$ and the brittle star Ophiomyxa brevidentis had the highest value (-4.31\%o \pm 1.20). The small starfish Henricia sp. had the lowest mean nitrogen value (3.86\% \pm 0.94 ) and the larger starfish Paranepanthia aucklandensis had the highest value of $9.92 \% \pm 1.17$. Opportunistic feeders/omnivores at the Bounty Islands (molluscs, crustaceans and echinoderms) showed little variability in their carbon signatures. Crustaceans' mean carbon values ranged from the lowest mean carbon value of $-18.84 \% \pm 0.35$ for the amphipod Talorchestia to the highest from an unidentified starfish $(-16.61 \%$ o \pm 0.48$)$. A wide range was found in the mean nitrogen for opportunistic feeders/omnivores with the lowest value of Henricia $(7.30 \%$ ะ 0.41$)$ and Talorchestia was considerably more enriched and variable in mean nitrogen $(21.40 \%$ \pm 3.37$)$.

\section{Invertebrate Predators}

Marine invertebrate predator mean carbon values were the lowest for the predatory whelk Fusitriton laudandus $(-19.44 \%$ ะ 0.39) and highest for the giant masking crab Leptomithrax australis (-18.48\%o), although there was little variation between samples in this group. Marine invertebrate predators showed variability in their mean nitrogen values with the least enriched being Fusitriton laudandus (6.41 \% \pm 0.69 ) and the most enriched being Leptomithrax australis (10.29\%o).Marine invertebrate predators at the Bounty Islands showed little variability in their mean carbon values. Leptomithrax australis was the most depleted in mean carbon $(-18.84 \% \pm 0.35)$ and the nudibranch Janolus ignis had 
the highest carbon value of -17.39. Marine invertebrate predators did not show a large range in their mean nitrogen values. Janolus ignis had the lowest nitrogen value (4.45). Leptomithrax australis was the most enriched of the predators and had a mean nitrogen value of $9.81 \%$ o \pm 1.51 .

\section{Fish}

Bovichtus variegatus (thornfish) was the only fish species sampled at Antipodes Island, and had a carbon value of $-19.49 \%$. No fish were sampled from the Bounty Islands.

\section{Seabirds}

Marine birds showed little variation in their mean carbon values at Antipodes Island. The petrel Pterodroma sp was the most carbon depleted $(-21.84 \% \pm 0.96)$. Separate skin samples of Antipodes albatross Diomedea antipodensis had the highest carbon values of $18.21 \%$ and-19.02\% \pm 0.65. Marine birds showed considerable variation in their mean nitrogen values; the lowest enrichment value was found for erect-crested penguin Eudyptes sclateri $(7.83 \%$ ๓0.96) and the highest for Diomedea antipodensis skin (16.33\%o). Bounty Islands’ marine birds showed considerable variation in carbon values between species. Eudyptes sclateri was the most depleted in mean carbon (-20.46 \% \pm 0.92$)$ and nonbreeding adult Salvin's albatross Diomedea cauta salvini had the highest mean carbon value of $-15.96 \% \pm 2.32$. Notable was the difference in carbon signature between samples from a recently deceased Diomedea cauta salvini and from non-breeding adults (difference of 3.90\%).The marine birds showed considerable variability in mean nitrogen values with Eudyptes sclateri having the lowest mean nitrogen value of $9.13 \%$ o \pm and the non-breeding adult Diomedea cauta salvini having the most enriched and variable values (17.91\% \pm $5.28)$.

\section{Marine Mammals}


There was no major difference between the two marine mammals sampled at Antipodes Island, with the mean carbon value of the elephant seal Mirounga leonine being slightly more depleted $(-17.78 \% \pm 1.33)$ than the New Zealand fur seal Arctocephalus forsteri (-17.74\% \pm 0.20$)$. Arctocephalus forsteri had the lowest mean nitrogen value of the two species (12.20 \% \pm 0.79) and Mirounga leonina the highest (15.02 \% \pm 0.66 ). Arctocephalus forsteri at the Bounty Islands displayed a small range in mean carbon values between pup skin samples and adult fur samples of $0.85 \%$; adult fur was more depleted than pup skin samples (-18.82 \% \pm 0.72$)$. No major variation was found between Arctocephalus forsteri pup skin samples and adult fur samples (0.37\%o). Arctocephalus forsteri pup skin samples were more enriched in mean nitrogen than the fur samples (13.27\% \pm 0.54$)$.

\section{Terrestrial Plants}

For terrestrial plants, the most depleted mean carbon value was found for a fern (-26.66\% \pm 0.37$)$ and the highest for a lichen (-18.36\% \pm 1.43$)$. A single species of lichen (unidentified) was the most varied between samples and the least enriched in mean nitrogen $(5.73 \%$ o \pm 5.18$)$. The highest mean value (noting large variability between samples) was found in the plants collected around the Anchorage Bay penguin colony (22.51\% \pm 6.27 ). There are no plants growing on the Bounty Islands apart from some small patches of Cook's scurvy grass that were not observed during this study, however there is a often a thin covering of algae on the rocks, which was found to have a mean carbon value of $-18.39 \%$ \pm 0.87 and a nitrogen value that was highly enriched and variable (16.76\%o $\pm 6.28)$.

\section{Terrestrial Invertebrates}

On Antipodes Island, terrestrial invertebrate mean carbon values ranged from $21.86 \%$ \% \pm 0.37 (flies) to $-14.07 \%$ o (worms). Terrestrial invertebrates also showed large variation between species with worms having the lowest nitrogen values (12.46\%) and flies the highest (25.27\%o \pm 1.80$)$. Spiders (Pacificana) were the only terrestrial invertebrates that were collected on the Bounty Islands and had a 
mean carbon value of $-18.11 \%$ o \pm 0.14 and mean nitrogen of $28.10 \%$ o \pm 0.81 . The only other terrestrial insects sampled were mosquitoes, which had a mean carbon value of $-18.78 \%$ o \pm 0.50 and mean nitrogen value of $22.84 \% \pm 2.09$.

\section{Terrestrial Birds}

The mean carbon values for terrestrial birds ranged from $-24.01 \%$ o \pm 1.89 in the red-crowned parakeet Cyanoramphus novaezelandiae hochstetteri to $-22.45 \%$ 土 0.38 in the snipe. Cyanoramphus novaezelandiae hochstetteri had the lowest mean nitrogen enrichment (10.12 \% \pm 0.19$)$ and the Antipodes parakeet Cyanoramphus unicolor the highest mean nitrogen enrichment (16.18\% \pm 0.52$)$.

\section{Terrestrial Mammals}

The only terrestrial mammal present on Antipodes Island is the introduced house mouse Mus musculus, which had a carbon value of $-18.93 \%$ o \pm 0.65 and a mean nitrogen value of $9.76 \%$ o \pm 1.69 .

\subsubsection{DIFFERENCE IN $\delta^{13} \mathrm{C}$ AND $\delta^{15} \mathrm{~N}$ BETWEEN SITES}

Where the same species was collected from two sites within the same island group, two-tailed t-tests were used to determine if any site effect was evident in the carbon and nitrogen values.

There was no consistent trend in the differences in carbon values between sites at Antipodes Island (Table 2-4). There was a significant difference between the carbon values for Eudoxochiton between Windward Island and Orde Lees (Windward Island sample was significantly more depleted in carbon). Nitrogen values for this species at Orde Lees were significant higher than at Windward. There was no significant difference in the mean carbon and mean nitrogen values for macroalgae between sites (Table 2-4) (Two-tailed t-tests p>0.05). For the only tussock species (Poa litorosa) collected at two different sites, Anchorage Bay sample was significantly more depleted in carbon than that from Hut Point. 
In contrast, the nitrogen values for tussock at Anchorage Bay were significantly higher than for Hut Point.

There was no consistent trend in the values of carbon between sites at the Bounty Islands (Table 2-5). The mussel Aulacomya maoriana and green seaweed Chaetomorpha were more depleted in carbon at Ruatara Island than at Depot Island; in contrast the limpet Cellana strigilis had higher carbon values at Depot Island than Ruatara Island. There was no significant difference in carbon signature between islands for starfish and giant kelp Macrocystis pyrifera. Red macroalgae were more depleted in carbon at Depot Island than at Tunnel Island.

In contrast, there was some difference in the mean nitrogen values between sampling sites. Species collected at Depot Island were significantly more enriched in nitrogen than the same species collected at other islands. There was no significant difference between the carbon values of starfish between Molly Cap and Proclamation Island.

\subsubsection{DIFFERENCE IN $\delta^{13} \mathrm{C}$ AND $\delta^{15} \mathrm{~N}$ BETWEEN THE BOUNTY AND ANTIPODES ISLANDS}

Two-tailed t-tests were used to determine if any difference in the carbon and nitrogen values existed between the Bounty and Antipodes Islands, where the same species was able to be collected from each island (Table 2-6). There was a clear pattern for macroalgae to be more depleted in carbon at Antipodes Island, with the exception of encrusting coralline algae that were not significantly different between islands. In contrast, the nitrogen values for macroalgae were significantly higher at the Bounty Islands than at Antipodes Island, other than coralline algae and the green alga Chaetomorpha that were not significantly different in nitrogen. Similarly, suspension feeders showed significantly lower carbon values at Antipodes Island than the Bounty Islands, with the exception of bryozoans that displayed lower carbon values at the Bounty Islands. The paua Haliotis virginea huttoni also displayed more depleted carbon values at Antipodes Island and higher nitrogen at the Bounty Islands, whilst the limpet 
Cellana strigilis showed no significant difference between the islands. The small starfish Henricia continued the trend, with depleted carbon at Antipodes Island and higher nitrogen at the Bounty Islands. Giant masking crab Leptomithrax australis did not show any significant difference in either carbon or nitrogen values between the islands. No significant difference was observed for fur seal Arctocephalus forsteri or erect-creasted penguin Eudyptes sclateri between islands.

\subsubsection{TROPHIC POSITIONS OF CONSUMERS}

Nitrogen values were used to determine trophic positions of consumers at Antipodes Island (Figure 2-7). The isotopic model suggested that Antipodes Island has six trophic levels. Primary producers, phytoplankton and macroalgae formed the base trophic level. Zooplankton were at the bottom of the trophic scale with the primary producers at one. Filter/suspension feeders, hydroids, sponges and mussels were located below the first trophic level with anemones, barnacles, and tubeworms occupying trophic positions between one and two. Grazers Haliotis virginea huttoni and chitons were found to have trophic positions between one and two, but limpets were elevated in their trophic levels in the upper portion of level two. Opportunistic scavenging gastropods and starfish had trophic levels between one and two with the starfish Paranepanthia aucklandensis, amphipods and brittle stars in the middle of level two. The only fish collected at Antipodes Island was also at the upper portion of level one. Predatory gastropods occupied trophic level one and two similar to the opportunistic scavenging gastropods. The giant spider crab Jacquinotia edwardsii had the highest trophic level of the predatory marine invertebrates at the upper end of trophic level two.

Both marine mammals, fur seal Arctocephalus forsteri and elephant seal Mirounga leonine, were at trophic level three. The only terrestrial mammal present on Antipodes Island, the introduced house mouse Mus musculus, occupied trophic level four. 
The lowest trophic level of all the birds at Antipodes Island was occupied by erect-crested penguin Eudyptes sclateri. Both skin and feather samples showed a trophic level in the mid to upper trophic level two. The remaining birds were positioned at levels four and five with the terrestrial parrot Cyanoramphus novaezelandie hochstetteri having the lowest trophic level, followed by the petrels, and at the top of the birds, the albatross Diomedea antipodensis was at level six. However, there was some variation in the trophic level of this species, with the feather sample suggesting a slightly lower trophic level of four. Of interest was the spread in trophic levels of the terrestrial birds that were all in the upper levels of the trophic scale, particularly the endemic parrot Cyanoramphus unicolor at the trophic level of six. Insects (fleas and ticks) occupied similar trophic positions to their hosts at trophic level three. At the top of the trophic scale for Antipodes Island were the flies collected in the penguin and fur seal colony.

The Bounty Islands had seven trophic levels (Figure 2-11). Primary producers, phytoplankton and macroalgae, formed the base of the trophic structure.

Secondary consumers were positioned in trophic levels below level one and up to level two, with bryozoans and sponges being within and below level one and anemones, mussels and barnacles having higher trophic positioning in the middle of level one. Paua Haliotis virginea huttoni was positioned as the lowest of the grazers within the upper range of level one. Amongst the grazers, limpets, chitons, and other gastropods were highly elevated with trophic positions of two for limpets and other gastropods and three for chitons. The opportunistic scavengers shrimps and starfish held similar trophic positions, at the upper and lower level two, but the amphipod Talorchestia was highly elevated as one of the highest species sampled at trophic level five. Predatory invertebrate crabs and predatory gastropods were found to have a trophic position of two whilst Janolus ignis, a nudibranch, was at trophic level one. Of the predatory species on the island the spider Pacificana was at the top of the trophic scale with a very high trophic level of seven. The birds sampled at the Bounty Islands were in three different trophic levels. The lowest was erect-crested penguin Eudyptes sclateri at trophic level two, the Salvin's albatross Diomedea cauta salvini chicks at level 
three and the non-breeding adult Diomedea cauta salvini had a trophic position of five. The fur seal Arctocephalus forsteri was positioned at trophic level three. 
Table 2-4 Comparison of carbon and nitrogen ratios at Antipodes Island sites.

\begin{tabular}{|l|l|l|}
\hline Species & t-test results - Carbon & t-test results - Nitrogen \\
\hline Eudoxochiton & Windward Island $<$ Orde & Orde Lees $<$ Windward \\
& Lees, $\mathrm{p}<0.05$ & Island, $\mathrm{p}<0.05$ \\
\hline Red seaweed & No significant difference & Ringdove Bay $<$ \\
& between Windward and & Windward Island, \\
& Ringdove Bay, $>0.05$ & $\mathrm{p}<0.05$ \\
\hline Moss & Anchorage Bay $>$ Hut & Anchorage Bay $<$ Hut \\
& Point, $\mathrm{p}<0.05$ & Point, $\mathrm{p}<0.05$ \\
\hline
\end{tabular}

Table 2-5 Comparison of carbon and nitrogen ratios at Bounty Islands sites.

\begin{tabular}{|c|c|c|}
\hline Species & t-test results - Carbon & t-test results - Nitrogen \\
\hline Aulacomya maoriana & $\begin{array}{l}\text { Ruatara Island }<\text { Depot } \\
\text { Island, } p<0.05\end{array}$ & $\begin{array}{l}\text { Depot Island }<\text { Ruatara } \\
\mathrm{p}<0.05\end{array}$ \\
\hline Cellana strigilis & $\begin{array}{l}\text { Ruatara Island }>\text { Depot } \\
\text { Island, } \mathrm{p}<0.05\end{array}$ & $\begin{array}{l}\text { Depot Island }<\text { Ruatara } \\
\text { Island, } \mathrm{p}<0.05\end{array}$ \\
\hline Chaetomorpha & $\begin{array}{l}\text { Ruatara Island }<\text { Depot } \\
\text { Island, } p<0.05\end{array}$ & $\begin{array}{l}\text { Depot Island }<\text { Ruatara } \\
\text { Island } \mathrm{p}<0.05\end{array}$ \\
\hline Macrocystis pyrifera & $\begin{array}{l}\text { No difference between } \\
\text { Lion Island and Tunnel } \\
\text { Island } p>0.05\end{array}$ & $\begin{array}{l}\text { Lion Island }<\text { Tunnel } \\
\text { Island } \mathrm{p}<0.05\end{array}$ \\
\hline Red seaweed & $\begin{array}{l}\text { Depot Island }<\text { Tunnel } \\
\text { Island }<0.05\end{array}$ & $\begin{array}{l}\text { Depot Island }<\text { Tunnel } \\
\text { Island }<0.05\end{array}$ \\
\hline Starfish & $\begin{array}{l}\text { No difference between } \\
\text { Molly Cap and } \\
\text { Proclamation Island } \\
\text { p }>0.05\end{array}$ & $\begin{array}{l}\text { No difference between } \\
\text { Molly Cap and } \\
\text { Proclamation Island } \\
\text { p }>0.05\end{array}$ \\
\hline
\end{tabular}


Table 2-6 Comparison of carbon and nitrogen ratios between the Bounty and Antipodes Islands.

\begin{tabular}{|c|c|c|}
\hline Species & t-test results - Carbon & t-test results - Nitrogen \\
\hline Adenocystis & $\begin{array}{l}\text { No significant difference } \\
p>0.05\end{array}$ & $\begin{array}{l}\text { No significant difference } \\
p>0.05\end{array}$ \\
\hline Anemones & $\begin{array}{l}\text { Antipodes Island } \\
<\text { Bounty Islands } \mathrm{p}<0.05\end{array}$ & $\begin{array}{l}\text { Bounty Islands }> \\
\text { Antipodes } \mathrm{p}<0.05\end{array}$ \\
\hline Arctocephalus fur & $\begin{array}{l}\text { No significant difference } \\
p>0.05\end{array}$ & $\begin{array}{l}\text { No significant difference } \\
p>0.05\end{array}$ \\
\hline Arctocephalus Scat & $\begin{array}{l}\text { No significant difference } \\
p>0.05\end{array}$ & $\begin{array}{l}\text { No significant difference } \\
p>0.05\end{array}$ \\
\hline Aulacomya maoriana & $\begin{array}{l}\text { Antipodes Island } \\
<\text { Bounty Islands } \mathrm{p}<0.05\end{array}$ & $\begin{array}{l}\text { Bounty Islands }> \\
\text { Antipodes } \mathrm{p}<0.05\end{array}$ \\
\hline Balanus & $\begin{array}{l}\text { Antipodes Island } \\
<\text { Bounty Islands } \mathrm{p}<0.05\end{array}$ & $\begin{array}{l}\text { No significant difference } \\
p>0.05\end{array}$ \\
\hline Cellana strigilis & $\begin{array}{l}\text { No significant difference } \\
p>0.05\end{array}$ & $\begin{array}{l}\text { No significant difference } \\
p>0.05\end{array}$ \\
\hline Chaetomorpha & $\begin{array}{l}\text { Antipodes Island } \\
<\text { Bounty Islands } \mathrm{p}<0.05\end{array}$ & $\begin{array}{l}\text { No significant difference } \\
p>0.05\end{array}$ \\
\hline Coralline encrusting & $\begin{array}{l}\text { No significant difference } \\
p>0.05\end{array}$ & $\begin{array}{l}\text { Bounty Islands }> \\
\text { Antipodes } \mathrm{p}<0.05\end{array}$ \\
\hline Eudyptes sclateri feather & $\begin{array}{l}\text { No significant difference } \\
p>0.05\end{array}$ & $\begin{array}{l}\text { No significant difference } \\
p>0.05(p=0.51)\end{array}$ \\
\hline Eudyptes sclateri scat & $\begin{array}{l}\text { No significant difference } \\
p>0.05\end{array}$ & $\begin{array}{l}\text { Bounty Islands }> \\
\text { Antipodes } \mathrm{p}<0.05\end{array}$ \\
\hline Haliotis virginia huttoni & $\begin{array}{l}\text { Antipodes Island } \\
<\text { Bounty Islands } \mathrm{p}<0.05\end{array}$ & $\begin{array}{l}\text { No significant difference } \\
p>0.05\end{array}$ \\
\hline Henricia sp & $\begin{array}{l}\text { Antipodes Island } \\
<\text { Bounty Islands } \mathrm{p}<0.05\end{array}$ & $\begin{array}{l}\text { Bounty Islands }> \\
\text { Antipodes } \mathrm{p}<0.05\end{array}$ \\
\hline Lessonia brevifolia & $\begin{array}{l}\text { Antipodes Island } \\
<\text { Bounty Islands } \mathrm{p}<0.05\end{array}$ & $\begin{array}{l}\text { No significant difference } \\
\mathrm{p}>0.05\end{array}$ \\
\hline Macrocystis pyrifera & $\begin{array}{l}\text { Bounty }<\text { Antipodes } \\
\text { Island } \mathrm{p}<0.05\end{array}$ & $\begin{array}{l}\text { Bounty Islands }> \\
\text { Antipodes } \mathrm{p}<0.05\end{array}$ \\
\hline Marginariealla parsonii & $\begin{array}{l}\text { Antipodes Island } \\
<\text { Bounty Islands } \mathrm{p}<0.05\end{array}$ & $\begin{array}{l}\text { Bounty Islands }> \\
\text { Antipodes } \mathrm{p}<0.05\end{array}$ \\
\hline Unidentified brown & $\begin{array}{l}\text { Antipodes Island } \\
<\text { Bounty Islands } \mathrm{p}<0.05\end{array}$ & $\begin{array}{l}\text { Antipodes }>\text { Bounty } \\
\text { Islands } \mathrm{p}<0.05\end{array}$ \\
\hline Unidentified Red & $\begin{array}{l}\text { Antipodes Island } \\
<\text { Bounty Islands } \mathrm{p}<0.05\end{array}$ & $\begin{array}{l}\text { Antipodes }>\text { Bounty } \\
\text { Islands } \mathrm{p}<0.05\end{array}$ \\
\hline Leptomithrax australis & $\begin{array}{l}\text { No significant difference } \\
\mathrm{p}>0.05\end{array}$ & $\begin{array}{l}\text { No significant difference } \\
\mathrm{p}>0.05\end{array}$ \\
\hline Bryozoans & $\begin{array}{l}\text { Bounty }<\text { Antipodes } \\
\text { Island } \mathrm{p}<0.05\end{array}$ & $\begin{array}{l}\text { Bounty Islands }> \\
\text { Antipodes } \mathrm{p}<0.05\end{array}$ \\
\hline
\end{tabular}


Figure 2-4 Plot of carbon and nitrogen stable isotope ratios for samples from Antipodes Island.

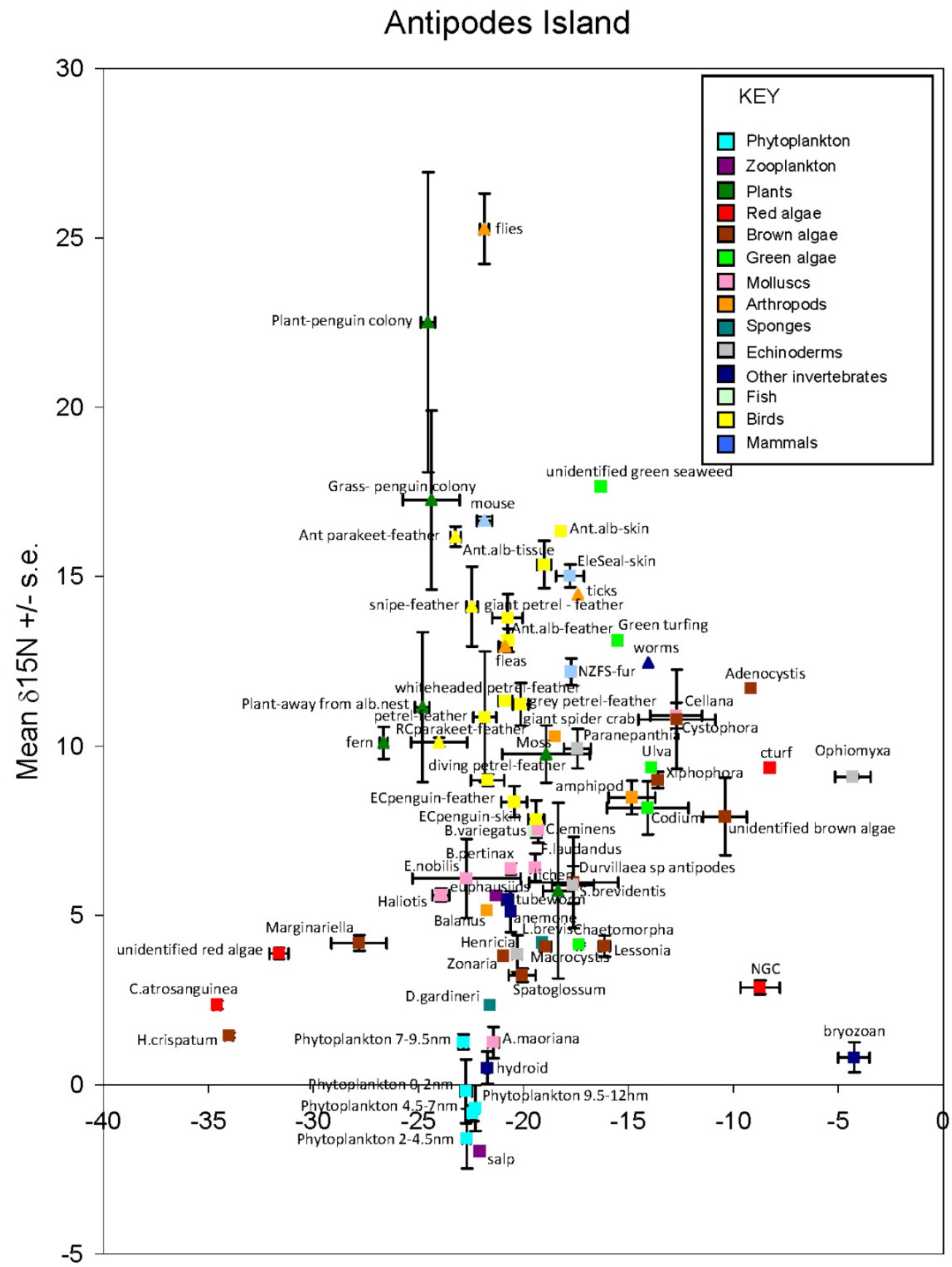

Mean $\delta 13 \mathrm{C}+/$ - s.e. 
Figure 2-5 Carbon ratios for Antipodes Island.

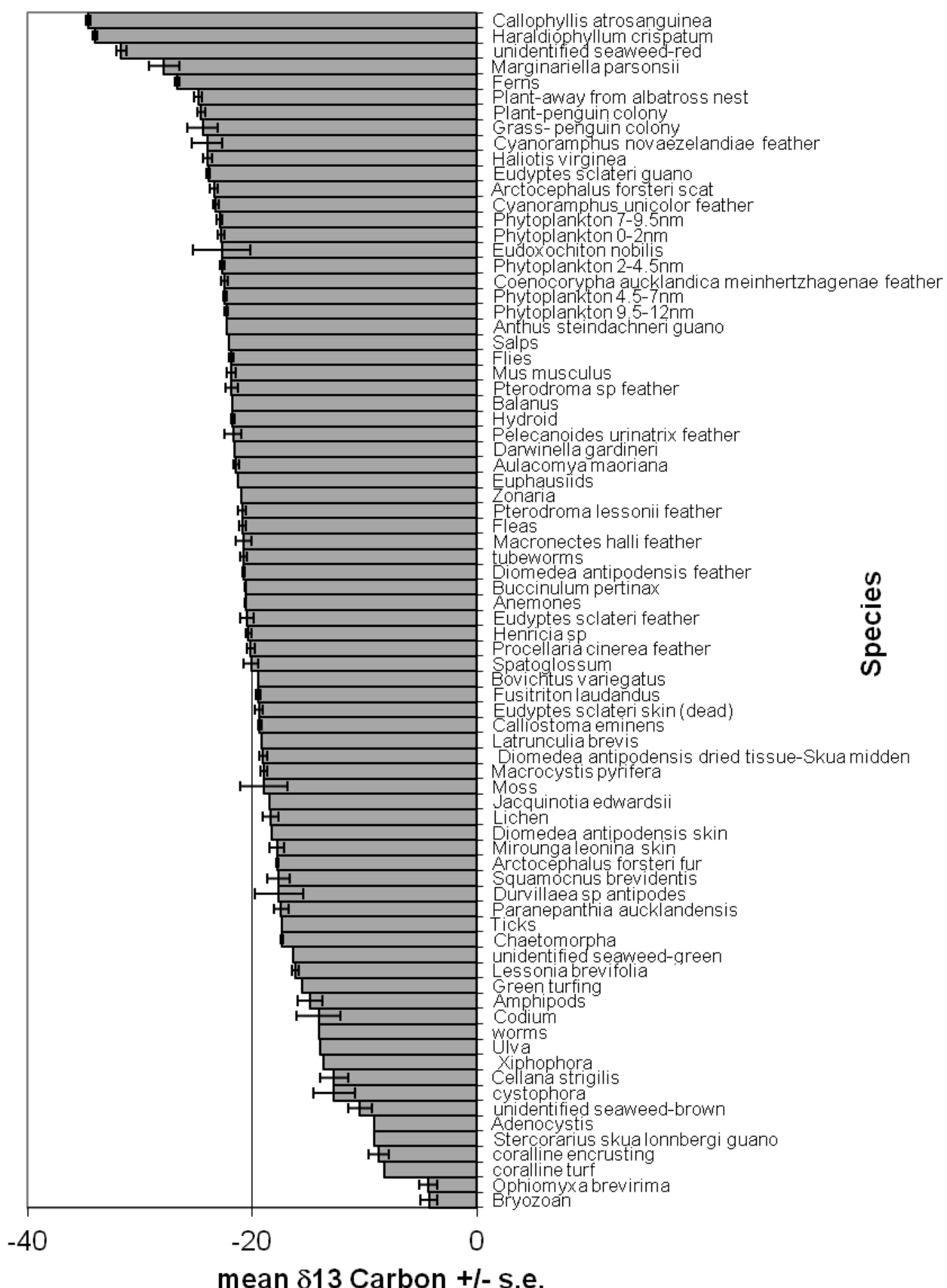


Figure 2-6 Nitrogen ratios for Antipodes Island.

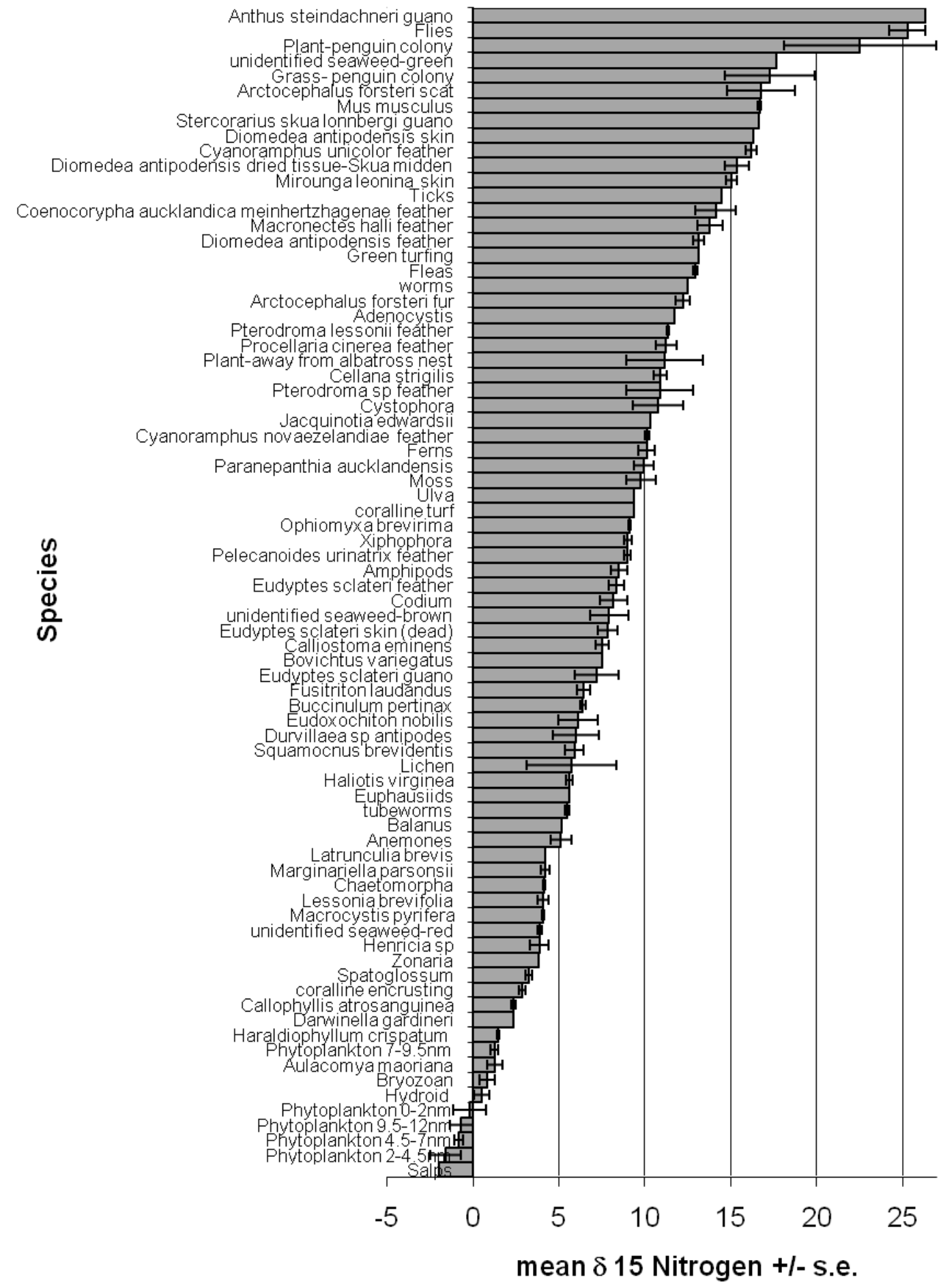


Figure 2-7 Trophic levels at Antipodes Island.

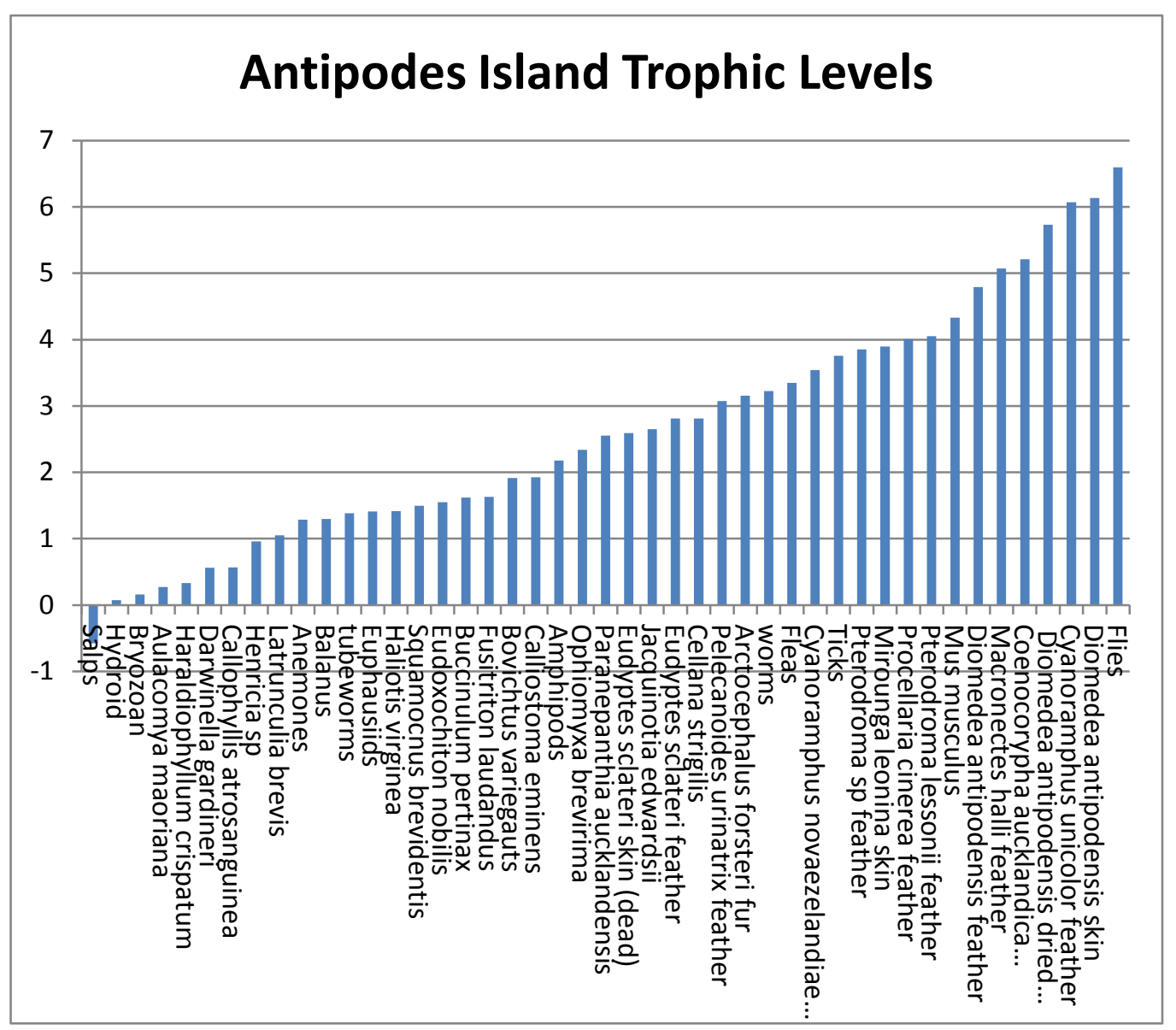


Figure 2-8 Plot of carbon and nitrogen stable isotope ratios for samples from the Bounty Islands.

Bounty Islands

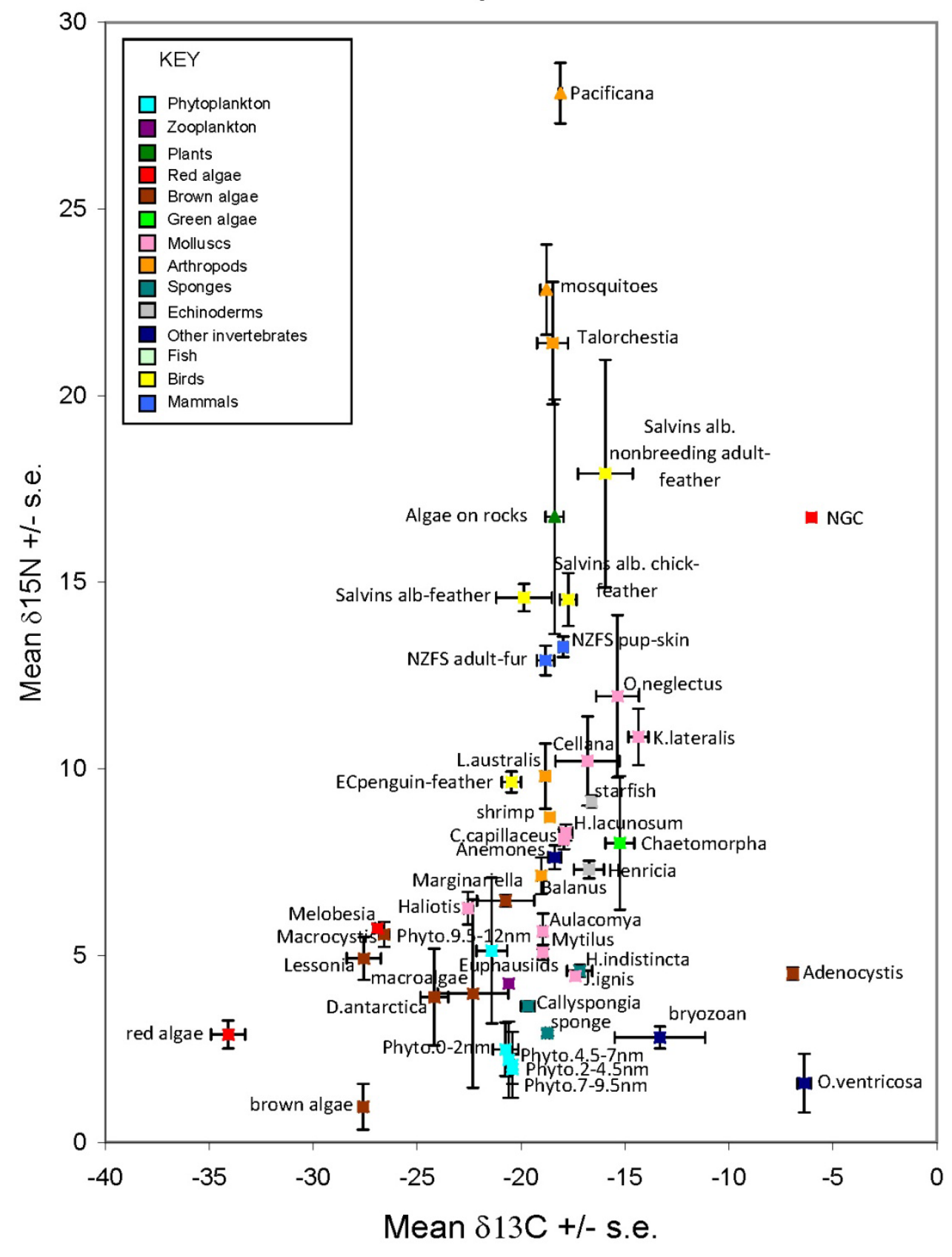


Figure 2-9 Carbon ratios for the Bounty Islands.

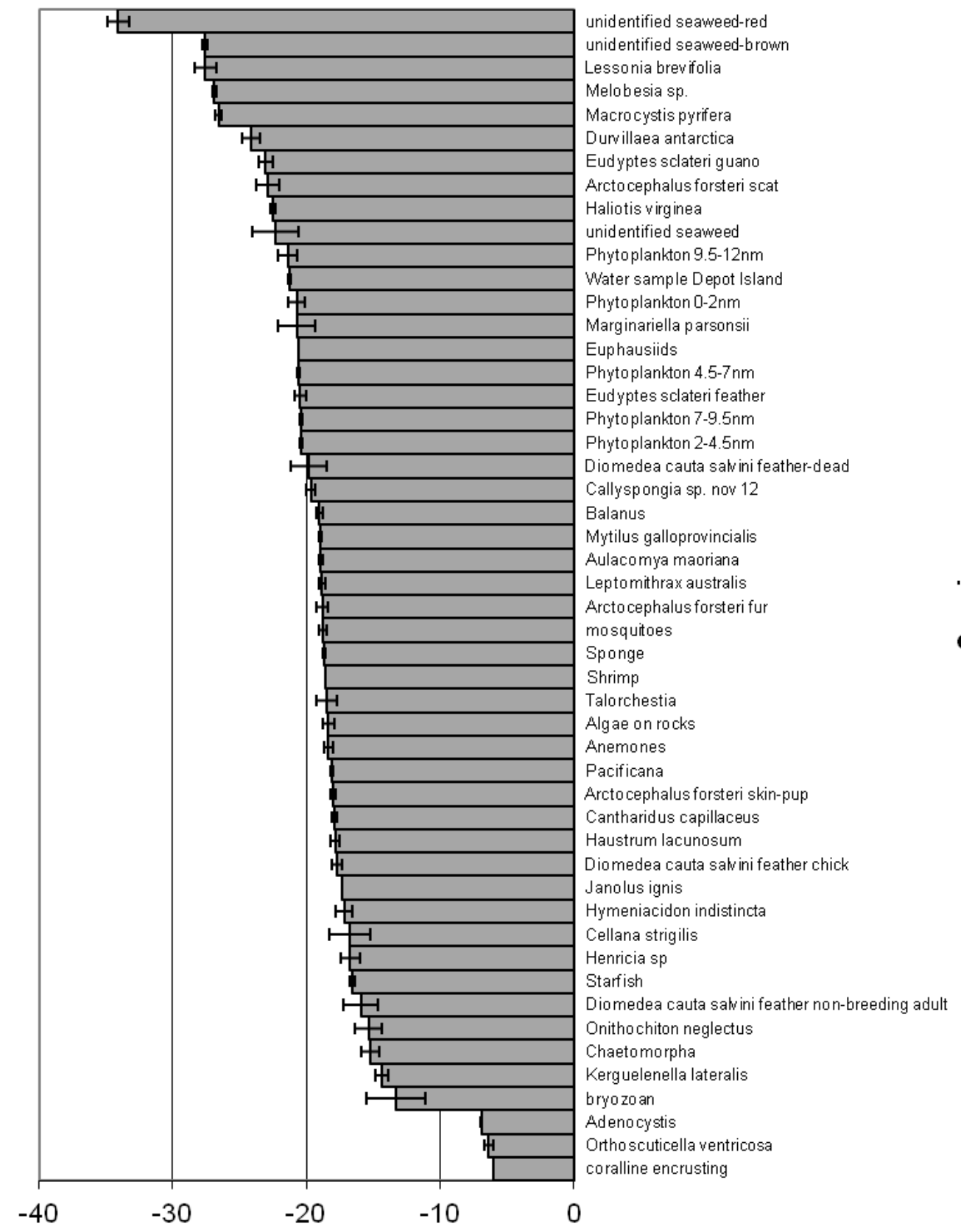

mean $\delta 13$ Carbon +/- s.e. 
Figure 2-10 Nitrogen ratios for the Bounty Islands.

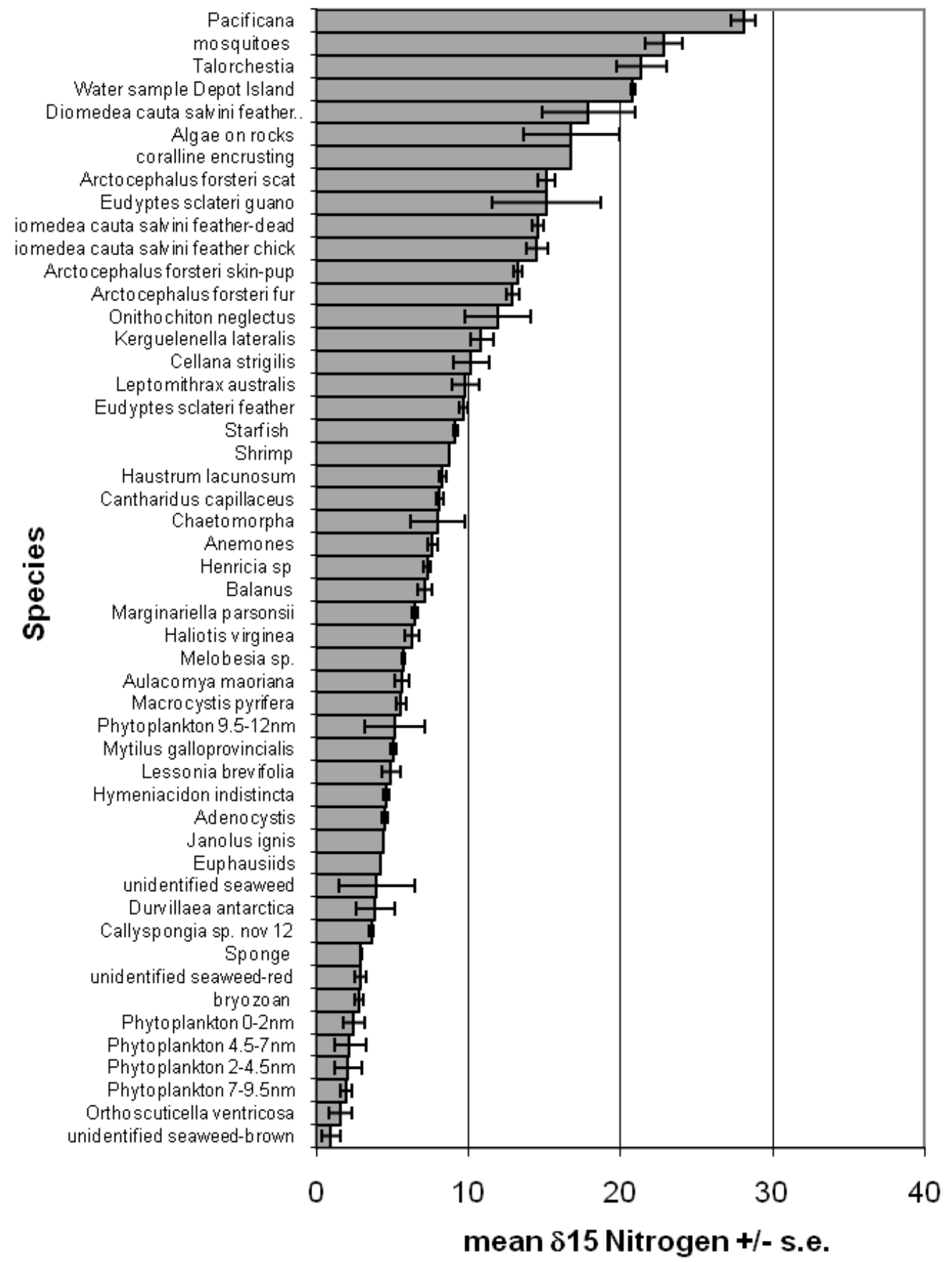


Figure 2-11 Trophic levels at the Bounty Islands.

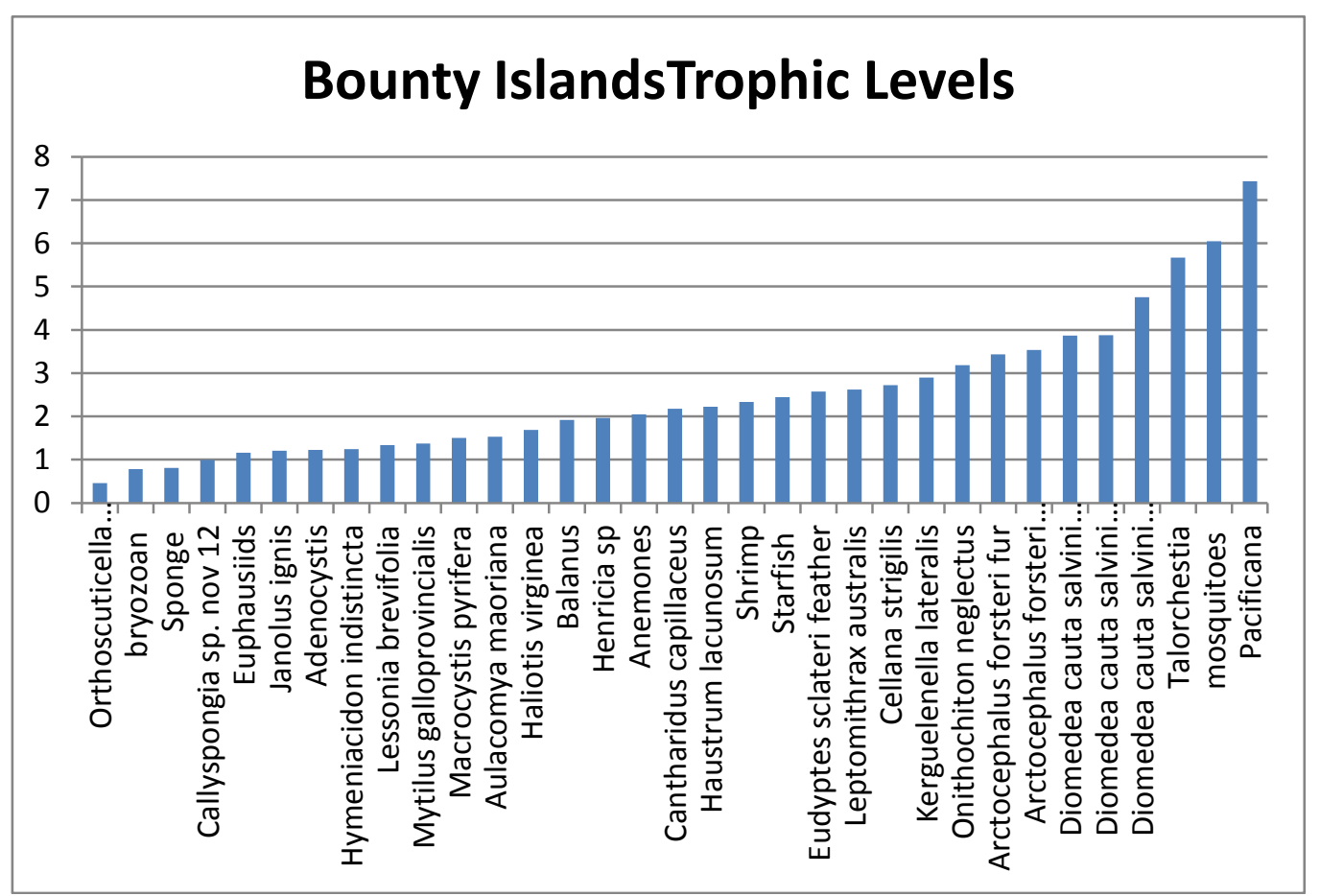




\subsection{DISCUSSION}

Understanding the trophic structure of food webs is an important initial step in determining how an ecosystem functions and provides valuable information on the linkages between species and habitats (Polis and Winemiller, 1996). It is critical for environmental managers to have an understanding of ecosystem architecture and how vulnerable an ecosystem is to disturbance, so that decisions can be made in the wider context of the ecosystem and not limited to a speciesby-species or habitat-by-habitat approach (Halpern et al., 2010).

Little was previously known about the Antipodes and Bounty Islands ecosystems and my study provides the first step in describing their trophic structure. The use of stable isotopes to describe the isotopic composition of the islands has revealed that both islands support a similar number of trophic levels, that may be elevated due to the inclusion of terrestrial species that prey upon or scavenge on marine species. Antipodes Island has more complexity in its food web compared to the Bounty Islands, which may indicate that the Bounty Islands could be more vulnerable to disturbance than Antipodes Island (Rooney et al., 2006). Omnivory was a feature at both of the islands, which is often prevalent in marine food webs (McCann, 2000, Polis and Strong, 1996, Link, 2002) and suggests that both islands have variability in their basal energy sources (McMeans et al., 2013).

\subsubsection{ANTIPODES ISLAND TROPHIC STRUCTURE}

Three different groupings of species were observed at Antipodes Island, based on their isotopic composition. The first group at the bottom of the trophic scale were sessile invertebrates that feed predominately on phytoplankton; the second consisted of omnivores and predatory invertebrates and the third was dominated by top level predators. The third group of species, including albatross, elephant seals and associated parasites, are known to have foraging strategies that are extensive in range and suggests that their isotope values are likely to be related to ecosystems outside of the influence of the island (Post et al., 2007a). 
Terrestrial plants showed the widest variation in nitrogen signatures, with plants within the penguin colony showing very high nitrogen values. It is likely that enrichment either directly via guano or via airborne ammonia may have contributed to the highly enriched nitrogen values, as this has been found in other studies of seabird nesting sites (Tatur and Myrcha, 1983, Erskine et al., 1998, Park et al., 2007). Similarly, terrestrial birds and the introduced house mice had carbon and nitrogen signatures between those for strictly terrestrial species and those for strictly marine species. This may indicate that the diets of terrestrial birds and mice on Antipodes Island are also enriched to some degree by marine species. Enrichment of terrestrial species by marine subsidies has been shown to occur within the vicinity of bird and marine mammal colonies (Polis and Hurd, 1996, Linderboom, 1984, Hawke and Miskelly, 2009), and will be the focus of Chapter Three.

Antipodes Island can be characterised as a complex food web with a complex trophic structure consisting of six trophic levels, which is higher than reported by other studies undertaken in the marine environment at high latitudes where marine mammals and sea birds were included in the food web (Dunton, 2001, Hobson and Welch, 1992). However, the marine species were found to have trophic positions from one to five, which is consistent with a review by Thompson et al. (2007) where it was shown that trophic levels in the marine environment frequently number up to five. Relationships between consumers and prey appeared inconsistent with trophic level status at Antipodes Island. Whilst there was a predictable step-wise trend in signatures for the secondary consumers and the top level predatory birds and marine mammals, marine invertebrate opportunistic scavengers and predatory invertebrates displayed similar trophic levels, suggesting the invertebrate fauna at the islands is omnivore dominated. This result from Antipodes Island suggests that the strict trophic level concept may not, in all cases, capture the complex relationships within ecosystems (Paine, 1988, Polis and Strong, 1996, Zanden and Rasmussen, 1999, Post, 2002). For example, Thompson et al. (2007) found in a review of trophic level data that strict trophic positions could be assigned to a majority of taxa in a food web but where secondary consumers were abundant, strict trophic levels were less 
reliable in determining links between species. These authors further concluded that trophic linkages become more complex when omnivory is prevalent.

There is a surprising lack of information examining linkages between marine and terrestrial species trophic levels, however, parasites or terrestrial species that feed on marine species would be influenced by the consumed species' nitrogen and should be considered as food chain links. Thompson et al. (2013) found that adding parasites to a published energy flow food web increased the number of trophic links between species, the link strength and the length of the food chains. There are differing views in regards to what constitutes stability within an ecosystem, ranging from the views expressed by May (2001) that there was no evidence from his models that species diversity or strong links between species lead to enhanced stability, to the contrasting position of Polis and Strong (1996) that suggest that complexity in food webs drives food web dynamics which in part leads to better defence against trophic cascades. In a recent study in the Arctic, McMeans et al. (2013) suggested that variability in resource use by consumers is the biggest factor for stability in a highly pulsed primary production ecosystem. The results presented here suggest that for Antipodes Island, the marine and terrestrial linkages that are influenced by three basal energy channels of macroalgae, phytoplankton and terrestrial plants, may play a role in the island's overall stability.

\subsubsection{BOUNTY ISLANDS TROPHIC STRUCTURE}

There were two main groupings of species within the Bounty Islands' food web. The first group of species appeared to be clustered around planktonic carbon and nitrogen values suggesting that phytoplankton is more important in structuring the Bounty Islands' marine community. This is consistent with the findings of Freeman et al. (2011) who found a higher percentage cover of filter feeding species at the Bounty Islands compared to Antipodes Island. These finding are also similar to those of Jacob et al. (2006) who found in a trophic study of Bouvet Island that the islands were strongly influenced by plankton as opposed to macroalgae. The second grouping at the Bounty Islands included the sea 
birds and marine mammals, that displayed a shift from the lower trophic level species indicating that their food source may be derived from areas outside the influence of the island. For example Cherel and Hobson (2007) showed in their stable isotope study of penguins foraging in the Southern Ocean, that penguins displayed a latitudinal gradient in their isotope signatures in relation to foraging sites, indicating that some species of the penguins were foraging in oceanic waters and others around the Subantarctic Islands. In an similar study on albatross in the Southern Ocean, Cherel et al. (2012) found that non-breeding Salvin's albatross (present on the Bounty Islands) were not foraging in the Southern Ocean but had isotope signatures that indicated foraging locations in warmer waters not associated with the Southern Ocean.

The Bounty Islands' food web consisted of seven trophic levels with the upper trophic levels being occupied by insects that feed on marine mammal tissue. The Bounty Islands' food web lacks the complexity of the Antipodes Island food web. This could be due to the lack of terrestrial species, apart from algae and insects, and the lower diversity in its marine species (Freeman et al., 2011). Relationships beyond secondary consumers and top predators at the Bounty Islands showed a generalist feeding pattern. Opportunistic scavengers, grazers and predators displayed mixed trophic levels between one and three, with no apparent step-wise trend in feeding patterns, suggesting that the Bounty Islands has a high level of omnivory. Whilst the trophic level model may not capture all the complexities of the food web at the Bounty Islands, it was still useful in showing the relative position of consumers in the food chain. Top-level marine birds and marine mammals were predictably towards the top of the trophic scale. At the highest trophic levels were mosquitoes and the endemic spider Pacificana - both species were highly enriched in nitrogen and with carbon values similar to fur seals. The Bounty Islands have no plant species and therefore all insects are reliant on marine based nutrients (see Chapter 3 for more details). 


\subsubsection{VARIATION WITHIN AND BETWEEN ISLANDS}

The mean carbon and nitrogen values of each island showed that species at the Bounty Islands were significantly more enriched in nitrogen and more depleted in carbon than at Antipodes Island. Differences between the islands could be due to a range of factors, including geographical variation in stable isotope signatures, although geographical variation is more evident in higher trophic levels as found by Cherel and Hobson (2007). Lower carbon base values transferred up the food chain suggest that Antipodes Island may be more influenced by oceanic waters than the Bounty Islands - the Bounty Islands are positioned in shallower waters, whereas Antipodes Island is situated on the edge of the continental self in close proximity to deep water (3000 m) and experiences different currents to the Bounty Islands (Booth, 2004). The higher nitrogen values of species at the Bounty Islands may also be due to the enrichment caused by high density colonies of sea birds and marine mammals excreting large amounts of guano. Large inputs of nitrogen from external sources have been shown to enrich receiving aquatic communities (Kolb et al., 2010), and could play an important role in driving the significant difference in nitrogen values between the islands.

The Bounty Islands lacks the complexity and some components in its food web compared to the Antipodes Island. There appears to be a major group of species dependent on phytoplankton either directly or re-circulated through top predator excrement, with little apparent influence from macroalgae. The higher and lower trophic levels at the Bounty Islands are coupled but the links do not appear to be as strong as at Antipodes Island, which are based around a higher diversity of species that use terrestrial and marine based food sources. These results suggest that the Bounty Islands terrestrial and marine environment may be more susceptible to disturbance than the Antipodes.

There were significant differences in nitrogen values between the Bounty Islands sites. Depot Island nitrogen values were consistently higher than those of other islands. This may be explained by the extremely high abundance of seabirds and marine mammals on Depot Island having an enrichment factor on these species. 
This is consistent with other research showing that species in the vicinity of high density colonies are more enriched in nitrogen than those that are further from colonies (Erskine et al., 1998).

\subsubsection{LIMITATIONS AND BENEFITS OF THIS STUDY}

This study presents a snapshot in time of the food webs of Antipodes and the Bounty Islands. The use of stable isotope analysis has been a useful tool for tracking mass energy flow within the limited timeframe for sampling and provides a baseline for future studies of food web structure or climate change effects in the region. The intent of this study was to collect a wide range of the most common species at each island which I was successfully able to complete. However, I was unable to (due to time restrictions) collect samples of species that are more difficult to sample, particularly at the Bounty Islands where samples of nearshore foraging birds such as Bounty Island shags and terns could not be collected due to their nesting locations on cliffs. I was also unable to find wetas and moths from the Bounty Islands that would have completed a full set of terrestrial invertebrates (Peat, 2003). Fish species at the Bounty Islands proved difficult to catch using conventional rod and reel and fish trap methods and are therefore absent from my data set.

The lack of freezer storage was also an issue on the vessel and I was unable to freeze large volumes of material. However, the use of $70 \%$ ethanol to store macroalgae had no detectable effect on their stable isotope values and therefore could be used in future studies if limited freezer space is available. More laboratory testing of this method would be useful to further examine if the length of time samples are stored in ethanol has any effect on isotope values.

This study has demonstrated that the use of stable isotopes is an effective method for undertaking time or sampling restricted research. The method has also been effective in determining the food web structure at the Antipodes and Bounty Islands using low impact sublethal sampling methods on protected species and should be considered as an alternative to traditional methods such as tubing 
birds, forcing regurgitation and anesthetising marine mammals used in other studies (Horne, 1985, Moore, 1997, Cherel, 2008).

\subsubsection{CONCLUSION}

The research presented in this study builds on the results of Freeman et al. (2011) and finds that despite the Bounty and Antipodes Islands being separated by only 100 nautical miles, they are very different ecosystems. Using stable isotopes to construct the food webs, calculate trophic levels and reconstruct diet, has provided some insight into the functioning of these ecosystems. Whilst it is clear that the islands are different, the question remains as to what is driving the differences. It may be that nutrient subsidies, water chemistry or currents and upwelling are factors determining how the two ecosystems function. These concepts will be explored in the following chapter. 


\section{CHAPTER THREE: Food web drivers at Antipodes Island and the Bounty Islands}

\subsection{INTRODUCTION}

The physical and biological processes that determine ecosystem structure and function are often highly variable both temporally and spatially (Barry and Dayton, 1991). Marine communities are structured by the productivity of the surrounding water, where nutrients, phytoplankton productivity, detritus and allochthonous input are key drivers (Polis et al., 1996). The flow of nutrients between habitats plays an important role in the energy flow from productive habitats to less productive habitats (Summerhayes and Elton, 1923, Witman et al., 2004). Cross-habitat exchanges can be categorised into three groups: (1) transport of nutrients and materials by physical processes (2) transport of nutrients and materials by biotic vectors: and (3) the movement of prey and consumers between habitats (Ellis et al., 2006, Polis et al., 1997). These exchanges in nutrients and organisms are particularly important for small islands where the marine and terrestrial food-webs may be influenced by allochthonous input from the marine environment (Polis and Hurd, 1996, Anderson and Polis, 1998).

Highly mobile species such as seabirds and marine mammals have been shown to be important transporters of nutrients that link the marine and terrestrial environments (Wainright et al., 1998). Seabirds and marine mammals are capable of depositing vast amounts of guano and faeces that heavily influences the animal derived nitrogen and carbon budgets in many habitats, and may supplement locally available nutrient sources. (Polis et al., 1997, Fariña et al., 2003). These subsidies are defined as a donor-controlled resource (prey, detritus, nutrients) from one habitat to another (plant or consumer) from a second habitat which increases population productivity of the recipient, potentially altering consumer-resource dynamics in the recipient system (Polis et al., 1997). They can play a pivotal role in shaping the recipient habitat in regards to the function, behaviour, abundance and density of organisms (Darimont et al., 2008). For 
example, McCauley et al. (2012) showed that on a remote pacific atoll, sea birds roosting on native trees fertilised the soil, increasing coastal nutrients and the abundance of plankton, attracting manta rays.

The New Zealand Subantarctic plateau is known to be generally low in phytoplankton biomass. Despite there being sufficient concentrations of nutrients for phytoplankton production, there are low levels of primary production because of the low levels of dissolved iron and its interaction with low light and silicates (Bradford-Grieve et al., 2003). Using satellite measurements, Murphy et al. (2001) found that there were localized phytoplankton concentrations around Campbell Island and the Bounty Islands. It is not known why these localised accumulations occur as the water surrounding the islands is of the same water masses studied by Bradford-Grieve et al. (2003). The relationship between marine subsidies and the effect on the nearshore marine and terrestrial environments has not been studied at the four main New Zealand Subantarctic Islands (Campbell, Auckland, Antipodes and the Bounty Islands). Seabird guano has long been known to be a fertilizer of nearshore environments and the enrichment of nearshore waters as a result of nutrients flowing from bird colonies has been associated with increased phytoplankton production at locations such as the islands off South Africa (Bosman and Hockey, 1986) and in the Pacific (McCauley et al., 2012).

Several studies have determined the role that kelp detritus plays in high latitude nearshore marine environments (Kaehler et al., 2006, Fredriksen, 2003, Bustamante and Branch, 1996). Kaehler et al. (2006) showed that particulate matter originating from kelp at the Prince Edward Islands was an important component of benthic consumers' diet. They also showed that kelp detritus was not limited to the vicinity of the algal bed but distributed widely via physical processes. There have been no studies at Antipodes and the Bounty Islands to determine if kelp derived detritus plays a role in ecosystem functioning, but large beds of macroalgae do exist at Antipodes Island (Hay et al., 1985, Horning, 1986) suggesting that kelp detritus may be important for consumers at this island. 
Stable isotope signatures were used to identify the incorporation of marine based nutrients in terrestrial birds on the Snares Islands, the most northern of the New Zealand Subantarctic Islands. Hawke and Holdaway (2009) found that a number of terrestrial birds' isotopic signatures were enriched with more marine-based signatures than others, suggesting that while some birds were consuming marine based nutrients derived from marine mammals and sea birds, others on the island had strictly terrestrial-based diets. This is important information for ensuring that any management planning recognises the full extent and importance of the food resources available to species in these high conservation value areas.

Water sampling for nutrients can be a useful method to provide information on the drivers in a food web, particularly where nitrogen, ammonia and phosphorous may limit or enhance primary production. Such sampling can be complementary to biological studies, can help determine the constraints or consequences of ecological interactions and also provide insights into the links between nutrients and ecosystem functioning (Elser and Urabe, 1999). Net primary production is limited by the availability of nutrients in the water column (Redfield, 1958, Ryther and Dunstan, 1971, Howarth, 1988, Vitousek and Howarth, 1991) and there is a strong body of evidence that suggests that excrement input by seabirds and marine mammals is a valuable fertilizer in the marine environment (Bosman and Hockey, 1986). Studies of the scale and dispersal of water nutrients have largely been limited to studies adjacent to rock pools and terrestrial sites around bird nesting or marine mammal breeding and haulout sites (Loder et al., 1996, Bosman and Hockey, 1986). The scale of water nutrient enhancement at Antipodes and the Bounty Islands are not known, but some recent studies of other islands that support populations of sea birds suggest that the spatial scale of enhancement may potentially be large (McCauley et al., 2012).

Stable isotope analysis provides an opportunity to identify the trophic structure of consumers (see Chapter 1). Measurements of single ratios of carbon can also be used to determine the possible contribution to a consumer's diet of two sources (Fry, 2006). The combination of both carbon and nitrogen can also be used to determine how multiple sources may contribute to a consumer's diet (Phillips and Gregg, 2003). Most studies that are undertaken to determine the 
role subsidised nutrients play in an ecosystem are based exclusively either in the marine environment or the terrestrial environment. McCauley et al. (2012) is one of the few studies that have attempted to define the terrestrial and marine interactions using a combination of both water nutrient concentrations and stable isotopes.

I hypothesise that the Antipodes Island food web will be influenced by macroalgae detritus and that the Bounty Islands food web will be influenced by nutrient subsidies from sea birds and marine mammals. The aims of this chapter are to quantify the possible ecosystem drivers behind the Antipodes and Bounty Islands ecosystems and to determine if the transport of marine nutrients is evident in terrestrial species at the islands. I will determine if there are any relationships between nutrient concentrations and distance from shore at the islands, if there are any differences in nutrient concentrations between Antipodes and the Bounty Islands and consider possible reasons for these differences. I will also use stable isotope signatures to determine what contribution phytoplankton, kelp and terrestrial food sources provide to the species of both islands using a simple mixing model. I will then further test the results of the mixing model using Isosource to determine the feasible diet of the five most abundant marine invertebrates at each of the islands and the feasible diets of several terrestrial bird and insect species. 


\subsection{MATERIALS AND METHODS}

Stable isotope and surface water nutrient samples were collected between March and April 2009 on an expedition to Antipodes and the Bounty Islands to describe the biodiversity of the marine environment (see General Introduction).

\subsubsection{PHYTOPLANKTON SAMPLING}

To determine if there was any correlation between distance from shore and concentration of nutrients or phytoplankton stable isotope signatures, periodic sampling in open ocean sites (beyond 12 nautical miles) and along two transects were undertaken at each island. A water sample was also collected from a stream of excrement from the Bounty Islands - this sample was not filtered due to the thick nature of the fluid but was analysed for stable isotopes as described in Chapter 2. Transect one started when entering the territorial sea of each island and transect two started from the coast when leaving the islands (Figure 3-1). Surface water was collected every 2.5 nautical miles from the edge of the territorial sea to the shore of the islands.

Seawater was collected using a plastic container from the stern of the vessel, stored in 3 separate 10 litre containers and then filtered as soon as possible following collection (usually within two hours). Where possible, three replicates were collected but due to the length of time required to filter the samples (up to two to three hours) and the requirement to complete other field sampling the replicates were reduced to two per site. Water samples of at least 5 litres were collected with a clean container that had been acid rinsed (10\% HCL) then rinsed twice with DI water, then rinsed three times with seawater prior to filling with the water sample, to remove residue from the previous samples. The containers were filled as soon as practical following cleaning and filtered immediately when possible. If the water had to be stored prior to filtering, the containers were covered in black plastic sheeting (to prevent further photosynthesis) and placed in a position on the vessel that would minimise any agitation in order to minimise cell lysis. 
Zooplankton and macrodetritus were removed from the water samples by prefiltering through a $200 \mu \mathrm{m}$ mesh. The water samples were then filtered through a pre-ashed $\left(450^{\circ} \mathrm{C} \mathrm{x} 4 \mathrm{~h}\right.$ in a muffle furnace) $25 \mathrm{~mm}$ glass fiber filter (GF/F) at low vacuum pressure (5 in. Hg vacuum). When still on the filtration system (Figure 2-3), 4 mls of $1 \mathrm{~N} \mathrm{HCl}$ was added to the filters and left for 2 minutes then rinsed with distilled water to remove impurities. After filtering and rinsing, filter samples were placed in labelled 6-well bibby plates and taped securely using masking tape to prevent movement of filters between the wells. All filters were frozen immediately following filtration and kept frozen at $-20^{\circ} \mathrm{C}$. For stable isotope analysis, the filters were dried at low temperature $\left(50-60^{\circ} \mathrm{C}\right)$ and stored in a dessicator prior to analysis using the same methods as described in Chapter 2

\subsubsection{SURFACE WATER NUTRIENTS}

To determine the concentrations of dissolved reactive phosphorous (DRP), nitrate (NO3-N) and ammonium (NH4-N), and to determine if there was any correlation between nutrient concentration and distance from the islands, samples were collected at the same sites as the phytoplankton samples (described above), in the open ocean and at the same sampling stations on the territorial sea transects. Three replicate water samples were collected by hand then stored in 8 $\mathrm{ml}$ tubes and frozen in liquid nitrogen until analysis. Water samples were also collected adjacent to and away from seabird nesting, marine mammal haul out and breeding sites, to determine if there were any differences in the concentrations of nutrients between sites (Figure 3-2, Figure 3-3). Nutrient concentrations were determined by NIWA using an auto-analyzer (model:Astoria), where the concentrations of phosphorous, nitrate, ammonium were analysed simultaneously. Following analysis the raw data was provided on a spreadsheet. The provider combined each of the three samples from each site into one sample to provide for more material to analyse - this unfortunately did not allow for means to be generated for each site. 


\subsubsection{STABLE ISOTOPE - MIXING MODELS}

To determine the contribution provided by primary producers to the diet of consumers, mean large brown kelp and phytoplankton stable isotope carbon ratios from each island were used to compare the difference in the contribution of these carbon sources to each island's food web. A basic mixing model (Phillips and Gregg, 2001) was used where the single isotope of carbon in consumers' tissues was used to determine the percentage contribution of the kelp and phytoplankton carbon sources to the base diet of the consumers, based on isotopic mass balance. A tissue discrimination factor of $0.5 \%$ was used to correct the ratios of carbon for each source prior to calculation (McCutchan et al., 2003). The equation is as follows:

$$
\begin{aligned}
f_{1}= & \frac{\delta^{13} \mathrm{C}_{\text {mix }}}{\delta^{13} \mathrm{\delta}_{1}-\delta_{2}} \underline{\delta^{13} \mathrm{C}_{2}} \\
& \delta^{3}=1-\mathrm{f}_{1}
\end{aligned}
$$

The equation expresses $\delta 13 \mathrm{C}$ of the consumer (with subscript mix) as a combination of the $\delta 13 \mathrm{C}$ of the two prey (subscripts 1 and 2), weighted by their diet fractions (f1 and f2). The diet fractions are subject to the constraint that they sum to 1 (Phillips, 2012).

To further test the contribution of kelp, phytoplankton and terrestrial material to consumer diets and determine if any marine derived nutrients were consumed by terrestrial species, Isosource software (version 1.3.1) was used to reconstruct the distribution of feasible source contributions to consumer diets, using $\delta^{13} \mathrm{C}$ and $\delta^{15} \mathrm{~N}$, with from 5 to 7 possible dietary components. For this analysis I included the five most abundant mobile subtidal marine invertebrate consumers at each of the Antipodes and Bounty Islands as reported by Freeman et.al. (2011) using randomised photo quadrats at depths between $10 \mathrm{~m}$ and $20 \mathrm{~m}$. At Antipodes Island, the most dense mobile invertebrates were: the topshell Cantharidus capillaceus (4.89 / $\mathrm{m}^{2}$ +/- 0.69); strawberry holothurian Squamocnus brevidentis (1.98 $\left.\mathrm{m}^{-2}+/-1.47\right)$; starfish Henricia spp (1.02 $\left.\mathrm{m}^{-2}+/-0.15\right)$; paua / abalone 
Haliotis virginea huttoni $\left(0.44 \mathrm{~m}^{-2}+/-0.13\right)$; and the starfish Paranepanthia aucklandensis (0.41 $\left.\mathrm{m}^{-2}+/-0.09\right)$. At the Bounty Islands, the most abundant mobile invertebrates were: an unidentified shrimp $\left(1.67 \mathrm{~m}^{-2}+/-0.34\right)$; starfish Henricia spp (0.88 $\left.\mathrm{m}^{-2}+/-0.16\right)$; whelk Haustrum lacunosum $\left(0.67 \mathrm{~m}^{-2}+/-0.14\right)$; paua Haliotis virginea huttoni $\left(0.66 \mathrm{~m}^{-2}+/-0.64\right)$; and the chiton Eudoxochiton nobilis $\left(0.54 \mathrm{~m}^{-2}+/-0.12\right)$. I was able to sample all these species for stable isotope analysis, with the exception of Eudoxochiton nobilis, where I substituted Onithochiton neglectus, and Haustrum lacunosum, where I substituted Calliostoma eminens.

\subsubsection{DATA ANALYSIS}

Statistical analyses were undertaken using SPSS (SPSS, 2006) and Microsoft Excel. Student T-tests were used to determine if there was any significant difference between two sites. A Pearson correlation coefficient was used to determine if there was any significant correlation between nutrient concentrations and distance from shore. For comparing between Antipodes and the Bounty Islands water nutrient concentrations recorded from all sites within 12 nautical miles of each island group were pooled. For a comparison between each island and open ocean sites, the open ocean sites were pooled. 
Figure 3-1 Map of water nutrient and phytoplankton stable isotope sampling sites in the open ocean and on 12 nautical mile transects at Antipodes and the Bounty Islands.

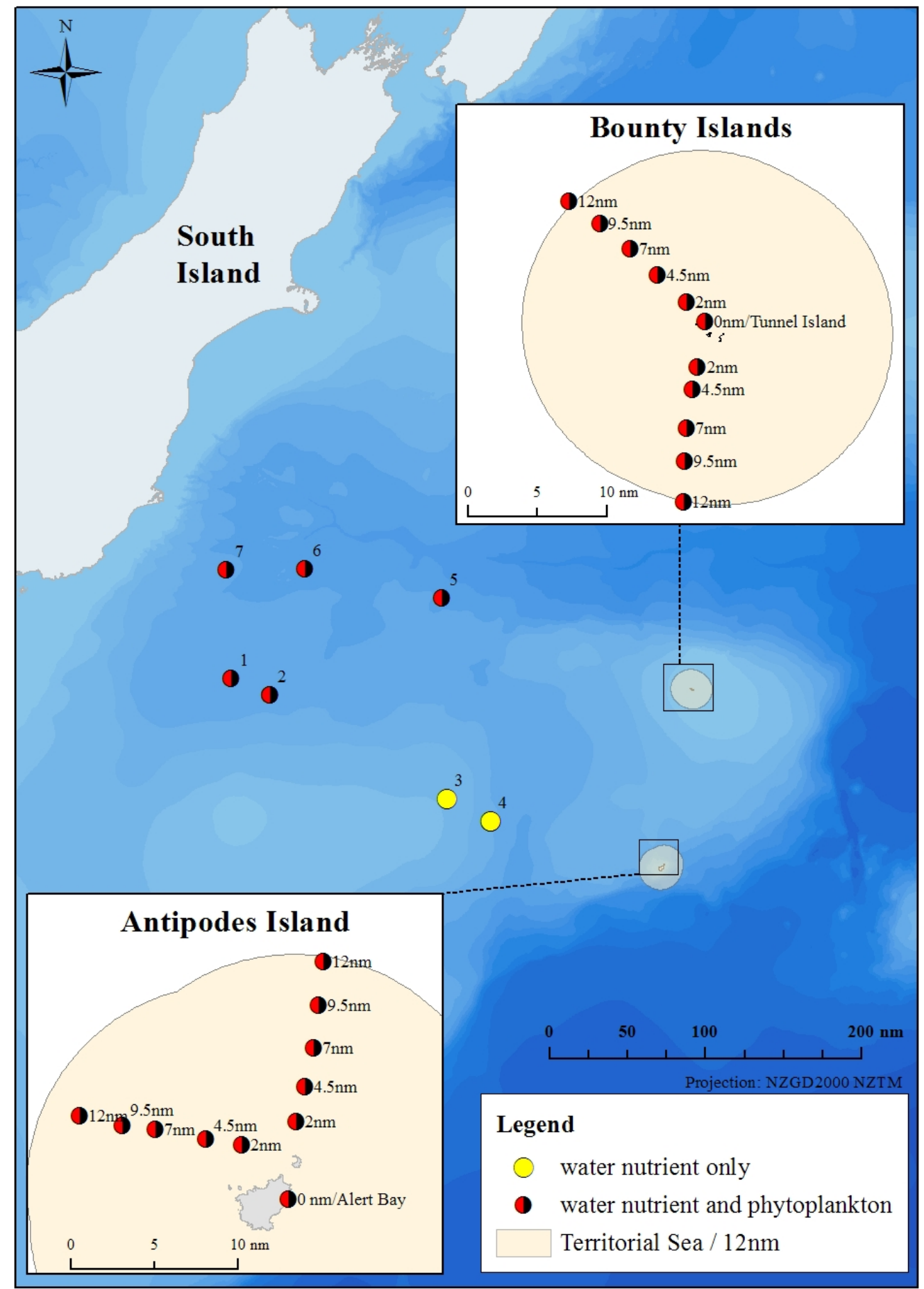


Figure 3-2 Water nutrient sampling and phytoplankton stable isotope sampling sites at Antipodes Island.

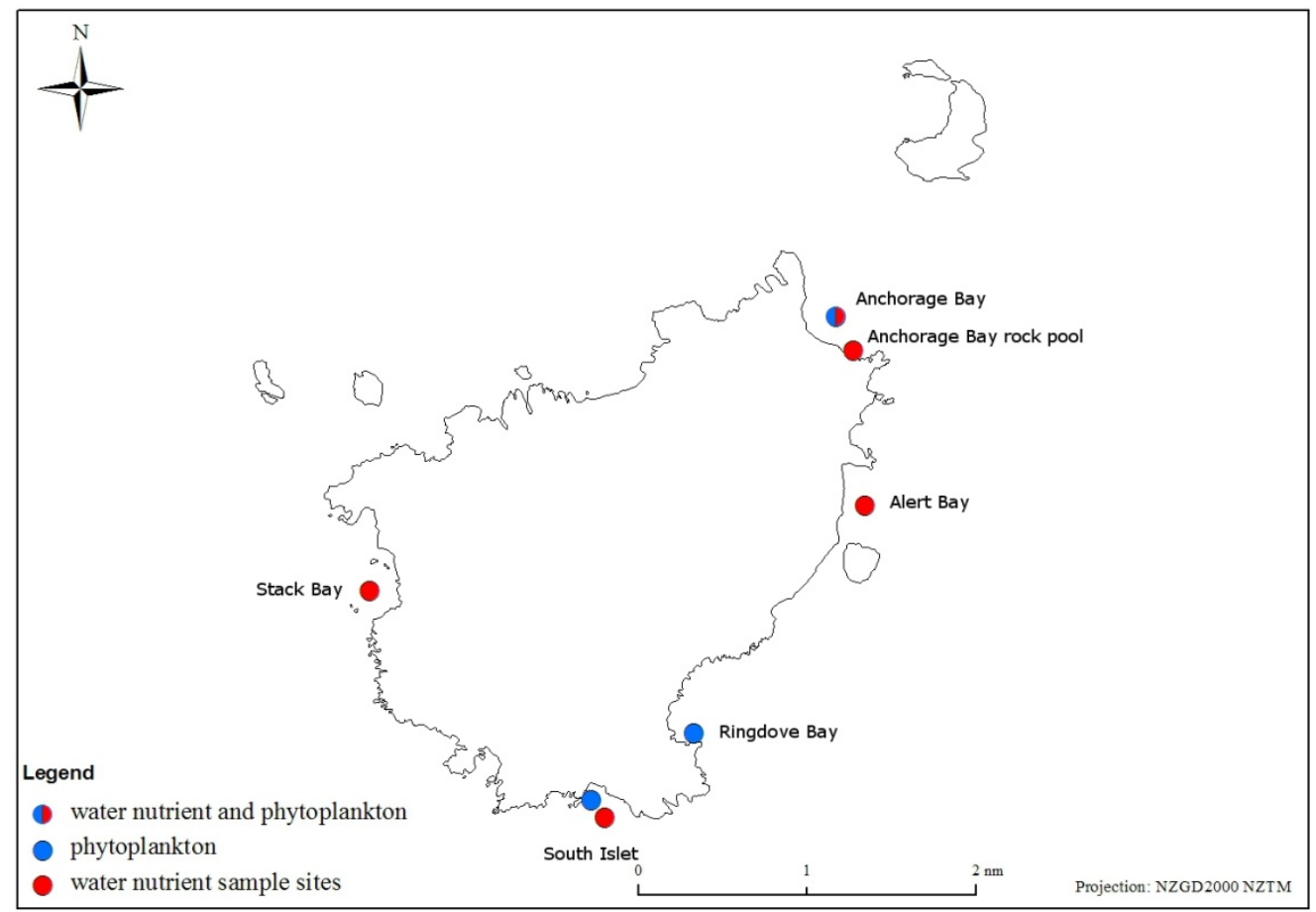

Figure 3-3 Water nutrient sampling and phytoplankton stable isotope sampling sites at the Bounty Islands.

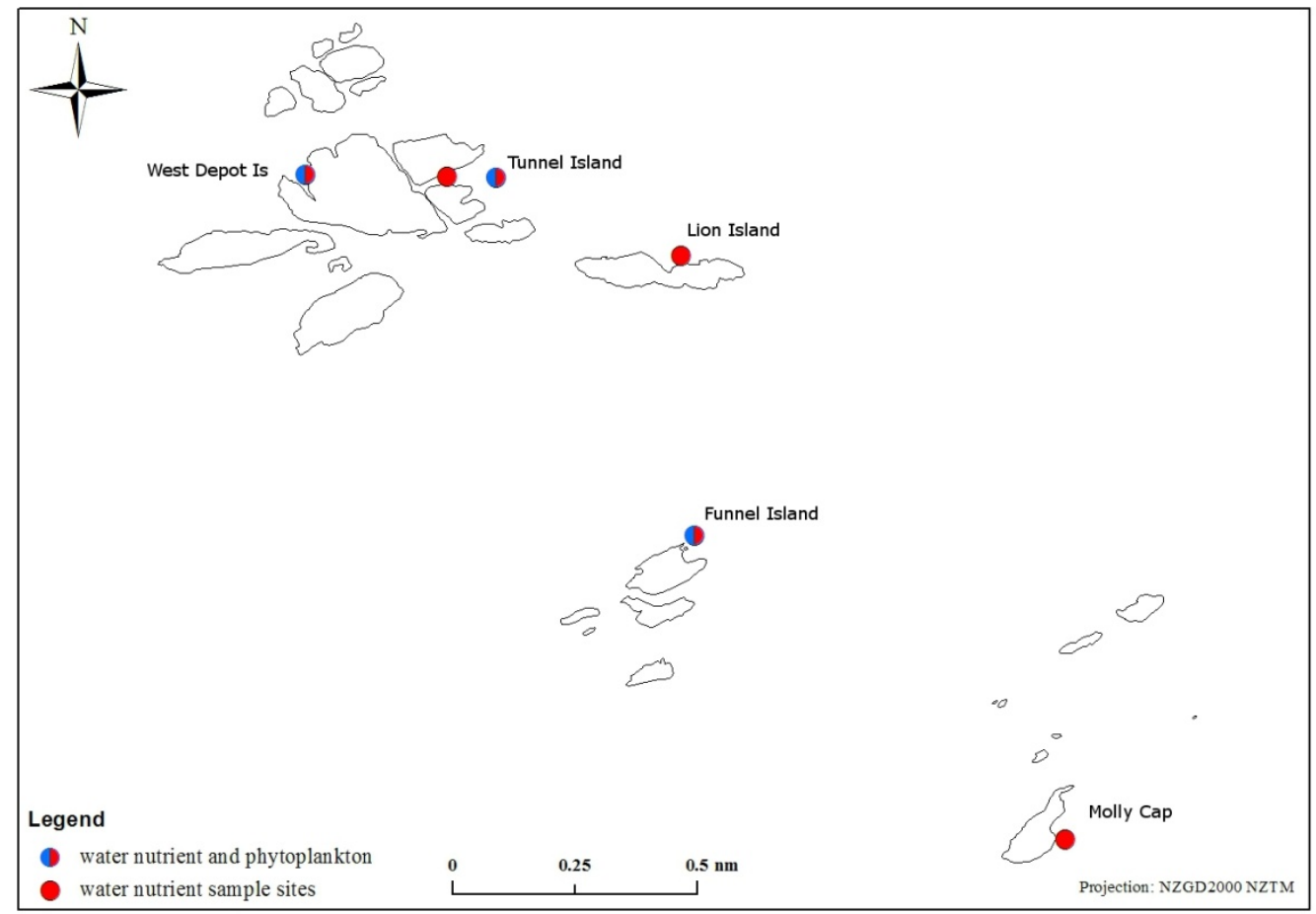




\subsection{Results}

\subsubsection{NUTRIENT CONCENTRATIONS}

Antipodes Island dissolved reactive phosphorous (DRP) ranged from a maximum concentration of $64 \mathrm{mg} \cdot \mathrm{m}^{-3}$ from an Anchorage bay rock pool to a minimum of $28 \mathrm{mg}^{-3}{ }^{-3}$ at South Islet (Table 3-1, Figure 3-4). DRP concentrations at the Bounty Islands had lower concentrations than the Antipodes with the maximum values reported from Tunnel Island $\left(38 \mathrm{mg} \cdot \mathrm{m}^{-3}\right.$ ) and the minimum values reported from $7 \mathrm{~nm}$ station on transect 2 (28 mg.m $\mathrm{m}^{-3}$ ) (Table 3-2, Figure 3-5). Open ocean samples of DRP ranged from the maximum at ocean open site 1 (53 mg. $\mathrm{m}^{-3}$ ) to the minimum at site 6 (25 mg. $\mathrm{m}^{-3}$ ) (Table 3-3, Figure 3-6).

The maximum concentration of $\mathrm{NH}_{4}-\mathrm{N}$ at Antipodes Island was collected in the rock pool in Anchorage Bay $\left(92 \mathrm{mg} \cdot \mathrm{m}^{-3}\right.$ ) and the minimum at the $4 \mathrm{~nm}$ station on transect 2 (Figure 3-4). Bounty Islands $\mathrm{NH}_{4}-\mathrm{N}$ was lower, with the maximum reported value from Molly Cap (28 mg. $\mathrm{m}^{-3}$ ) and the minimum from the $9.5 \mathrm{~nm}$ station on transect 1 (3 mg.m ${ }^{-3}$ ) (Figure 3-5). Open ocean $\mathrm{NH}_{4}-\mathrm{N}$ ranged from the maximum concentration at site $6\left(25 \mathrm{mg} \cdot \mathrm{m}^{-3}\right)$ and the minimum at site $1\left(5 \mathrm{mg} \cdot \mathrm{m}^{-}\right.$ ${ }^{3}$ ) (Figure 3-6).

$\mathrm{NO}_{3}-\mathrm{N}$ at Antipodes Island had the maximum concentration at the $2 \mathrm{~nm}$ station on transect $2\left(258 \mathrm{mg} \cdot \mathrm{m}^{-3}\right)$ and the minimum value at South Islet (116 mg.m $\left.\mathrm{m}^{-3}\right)$ (Figure 3-4). The Bounty Islands had lower values than Antipodes for $\mathrm{NO}_{3}-\mathrm{N}$, with the maximum collected from Tunnel Island $\left(226 \mathrm{mg}^{-3} \mathrm{~m}^{-3}\right.$ ) and the minimum from the 9.5nm station (172 mg.m ${ }^{-3}$ ) (Figure 3-5). Open ocean $\mathrm{NO}_{3}-\mathrm{N}$ ranged from the maximum concentration at open ocean site $4\left(260 \mathrm{mg} \cdot \mathrm{m}^{-3}\right)$ and the minimum at site 6 (152 mg.m ${ }^{-3}$ ) (Figure 3-6).

There was a no significant difference found between pooled sites at Antipodes Island and pooled sites at the Bounty Islands in DRP, $\mathrm{NH}_{4}-\mathrm{N}_{\text {or }} \mathrm{NO}_{3}-\mathrm{N}$ (Twotailed $t$-test, $\mathrm{p}>0.05$ ). Both the Antipodes Island and Bounty Islands nutrient 
concentrations within 12 nautical miles were significantly different to the open ocean samples (Two-tailed $t$-test, $\mathrm{p}<0.05$ ).

\subsubsection{PHYTOPLANKTON STABLE ISOTOPE ANALYSIS}

The highest Antipodes Island $\delta^{13} \mathrm{C}$ values were collected at Ringdove Bay (22.06) and the lowest at 2nm along transect 2 (-23.52) (Figure 3-7). The highest

$\delta^{15} \mathrm{~N}$ was recorded at Anchorage Bay (1.87) and the lowest from 2mn along transect 2 (-4.75) (Table 3-4). Bounty Islands $\delta^{13} \mathrm{C}$ values were higher than at the Antipodes Island with the least depleted sample being recorded from Tunnel Island (-18.90) and the most depleted sample from Funnel Island (-22.56) (Table 3-5, Figure 3-8). The lowest values at open ocean sites were collected at Site 1 (24.47) and the highest value was reported from site 4 (-19.34) (Table 3-6, Figure 3-9).

There was no significant difference found in the phytoplankton $\delta^{13} \mathrm{C}$ and $\delta^{15} \mathrm{~N}$ samples between Antipodes Island and the Bounty Islands (Two tailed Test, $\mathrm{p}>0.05)$. A significant difference was shown between the open ocean sites and both islands (Two-tailed $t$-test, $\mathrm{p}<0.05$ ).

\subsubsection{WATER NUTRIENT AND PHYTOPLANKTON TRANSECTS}

There was no correlation between the distance from Antipodes Island and DRP concentration (Table 3-7, Figure 3-4). At the Bounty Islands there was a negative correlation between DRP concentrations and increasing distance away from the islands (Table 3-7, Figure 3-5). There was no correlation between $\mathrm{NO}_{4}-\mathrm{N}$ concentration and distance from shore at either the Antipodes or Bounty Islands. $\mathrm{NO}_{3}-\mathrm{N}$ concentration at Antipodes Island displayed a positive correlation with distance from shore with $\mathrm{NO}_{3}-\mathrm{N}$ concentrations decreasing heading towards the island (Figure 3-7, Table 3-7). The opposite was recorded for the Bounty Islands, with $\mathrm{NO}_{3}-\mathrm{N}$ showing a negative correlation with distance away from the islands 
(Figure 3-5, Table 3-7). There was no correlation between nitrogen or carbon stable isotope signatures and distance for either Antipodes Island or the Bounty Islands (Table 3-7, Figure 3-7, Figure 3-8).

\subsubsection{CONTRIBUTION OF KELP, PHYTOPLANKTON AND TERRESTRIAL CARBON TO CONSUMER DIETS}

The range in the contribution of kelp and phytoplankton carbon to consumers' diets for the invertebrates at Antipodes Island was 0 to $100 \%$ for kelp and 0 to 100\% for phytoplankton (Table 3-8). At the Bounty Islands, kelp carbon contributed between 0 and 35\% and phytoplankton between 65 and 100\% of carbon to consumer diets (Table 3-9).

The diet of euphausiids at Antipodes Island consisted of 56\% kelp carbon and 44\% phytoplankton carbon, and Salps 7\% kelp and 93\% phytoplankton carbon. Diet of euphausiids at the Bounty Islands consisted of 100\% phytoplankton derived carbon.

Suspension feeders had mixed diets at Antipodes Island with the barnacle Balanus having the lowest kelp carbon contribution to their diet (29\%) and strawberry holothurians Squamocus brevidentis the highest kelp carbon contribution (100\%). The suspension feeder with the lowest phytoplankton carbon contribution to diet was anenomes (1\%) and Balanus the highest contribution (71\%). At the Bounty Islands all of the suspension feeders had a diet that consisted of $100 \%$ phytoplankton-derived carbon. At Antipodes Island, kelp carbon contributed $100 \%$ of the diet in the grazer Cellana strigilis. In contrast, phytoplankton carbon contributed 100\% to the diet of Eudoxochiton and paua Haliotis virginea huttoni at Antipodes Island. Amongst the opportunistic feeders/omnivores at Antipodes Island, kelp carbon contributed $99 \%$ to the diet of the whelk Buccinulum pertinax and $100 \%$ to the diet of all the echinoderms. At the Bounty Islands, all of the opportunistic feeders/omnivores and suspension feeders had a diet that consisted of $100 \%$ kelp derived carbon. The diets of all 
marine invertebrate predators at Antipodes Island consisted of $100 \%$ kelp carbon. At the Bounty Islands phytoplankton carbon contributed $100 \%$ to the diet of all marine invertebrate predators. Bovichtus variegatus (thornfish) was the only fish species sampled at Antipodes Island, and had a diet that consisted of 100\% kelp derived carbon. No fish were sampled from the Bounty Islands.

For the terrestrial environment at Antipodes Island, worms derived 100\% of their carbon from terrestrial sources. Flies derived 21\% of their carbon from kelp; fleas derived $82 \%$ of their carbon from kelp. Ticks’ diet consisted of $100 \%$ phytoplankton carbon. All of the terrestrial invertebrates collected at the Bounty islands had phytoplankton as their sole carbon source (100\%).

Terrestrial birds at Antipodes Island had a mixture of marine carbon and terrestrial carbon, with the diet of red-crowned parakeet Cyanoramphus novaezelandiae hochstetteri consisting of $86 \%$ phytoplankton carbon and $14 \%$ terrestrial carbon. Antipodes parakeet Cyanoramphus unicolor had a diet that had 49\% phytoplankton carbon and 51\% terrestrial carbon. Snipe diet consisted of $88 \%$ terrestrial carbon and $12 \%$ phytoplankton carbon. The only terrestrial mammal present on Antipodes Island is the introduced mouse Mus musculus of which $21 \%$ of its diet came from phytoplankton carbon sources and $79 \%$ terrestrial carbon.

\subsubsection{ISOSOURCE CASE STUDIES - MARINE INVERTEBRATES}

At Antipodes Island, bryozoan tissue contributed 9-17\% and the sponge Latrunculia brevis between $70-84 \%$ to the diet of strawberry holothurians Squamocnus brevidentis, (Appendix 1). Hydroids, other sponges, algae and phytoplankton did not play an important role in the diet of Squamocnus brevidentis. At the Bounty Islands, shrimp diet consisted of 5-37\% bryozoans and $0-30 \%$ run off from the island (Appendix 2). These values were very well constrained (small range between minimum and maximum) and demonstrates 
these items play an important role in the diet of shrimp, whereas barnacles, sponges and euphausiids were less constrained and therefore may not play such an important role in the diet of shrimps.

At Antipodes Island, Paranepanthia aucklandensis (starfish) showed a potential range of diet items, but bryozoans contributed 0-19\% and green seaweeds 0-24\% to its diet (Appendix 3). Limpets, sponges, chitons, tubeworms and gastropods may contribute to the overall diet but were less constrained. At the Bounty Islands, encrusting coralline algae plays an important role in the diet of the chiton Onithochiton neglectus, contributing 3-50\%. Guano run off from the island is also an important contributor to their diet at 0-35\% (Appendix 4). Green algae, bryozoans and red algae may play a role but results were less constrained and therefore they are likely to play a lesser role.

At the Antipodes the diet of starfish Henricia sp consisted of bryozoans 4-5\% and most importantly barnacles, 62- 90\% (Appendix 5). Macroalgae, sponges and gastropods were less constrained and did not play as important a role in their diet. At the Bounty Islands, Henricia sp diet consisted of 26-35\% bryozoans and 0-54\% sponges (Appendix 6). Chitons, barnacles, gastropods were not as an important contributor and less constrained in their values.

At Antipodes Island, red algae played an important role in the diet of paua Haliotis virginea huttoni, consisting of 0-56\% (Appendix 7). Sponges or other species with the same isotopic signature were also important in their diets (0$30 \%)$. Other macroalgae were present in the possible diet but values were less constrained. At the Bounty Islands, important contributors to the diet of Haliotis virginea huttoni were particulate organic matter (POM) (14-15\%), and red algae (40-68\%) (Appendix 8). Less important, but still possible contributors were the brown macroalgae Macrocystis pyrifera and Marginariella parsonii; other species of macroalgae played a less important role in its diet.

At Antipodes Island, 4-71\% of the diet of the tiger shell Calliostoma eminens consisted of the gastropod Fusitriton laudandus and 12-55\% of the sponge Latrunculia brevis (Appendix 9). Barnacles, mussels, other gastropods and 
tubeworms did not play as important a role, being less constrained in their values. At the Bounty Islands the diet of the whelk Haustrum lacunosum was less certain than other species and potentially consisted of a range of species - the mussel Aulacomya maoriana consisted of 2-24\% in the diet of Haustrum lacunosum and run off from the island 0-13\% (Appendix 10).

\subsubsection{ISOSOURCE CASE STUDIES: MARINE-TERRESTRIAL LINKAGES}

The diet of the endemic Antipodes parakeet Cyanoramphus unicolor consisted of $21-64 \%$ of prey items that are sourced from seal scats and 13-51\% from plant materials. Amphipods, penguin guano and the introduced house mouse were also possible features in their diet but the values were less constrained (Appendix 11). The diet of the red-crowned parakeet Cyanoramphus novaezelandiae hochstetteri consisted of 21-64 \% penguin guano and 13-51\% from plant material. Mice, seal scat and plant material were also possible contributors to their diet but values were less constrained (Appendix 12). The Antipodes Island snipe’s diet was mainly derived from terrestrial-based sources, with a particular shrub species making up 6-76\% of its diet and tussock/grasses contributing 7-53\%. Worms, penguin guano, kelp and amphipods were possible diet items but their values were less constrained (Appendix 13).

The diet of the introduced mice Mus musculus collected from Antipodes Island consisted of $0-51 \%$ grass material and $0-30 \%$ albatross tissue. Other plant material, petrel and seal tissue were also possible contributors to their diet but the values were less constrained (Appendix 14).

The Bounty Islands’ terrestrial endemic spider Pacificana had a diet that consisted of 5-11\% seal scat and 63-80\% other Pacificana spiders (i.e. cannibalism). Seal skin and fur, isopods and mosquitoes were also possible features in their diet but were less constrained in their values (Appendix 15). 
Table 3-1 List of water nutrient concentrations collected from Antipodes Island.

\begin{tabular}{|l|l|l|l|l|l|l|l|l|}
\hline Site & Transect & $\mathrm{n}$ & \multicolumn{2}{|c|}{ DRP } & \multicolumn{2}{c|}{$\mathrm{NH}_{4}-\mathrm{N}$} & \multicolumn{2}{c|}{$\mathrm{NO}_{3}-\mathrm{N}$} \\
\hline & & & $\mathrm{mg} \cdot \mathrm{m}^{-3}$ & $\mu \mathrm{m}$ & $\mathrm{mg}^{-3}$ & $\mu \mathrm{m}$ & $\mathrm{mg} \cdot \mathrm{m}^{-3}$ & $\mu \mathrm{m}$ \\
\hline $12 \mathrm{~nm}$ & 1 & 1 & 38 & 1.18 & 7 & 0.13 & 236 & 14.63 \\
\hline $9.5 \mathrm{~nm}$ & 1 & 1 & 37 & 1.15 & 9 & 0.16 & 237 & 14.70 \\
\hline $7 \mathrm{~nm}$ & 1 & 1 & 37 & 1.15 & 7 & 0.13 & 237 & 14.70 \\
\hline $4.5 \mathrm{~nm}$ & 1 & 1 & 41 & 1.27 & 8 & 0.14 & 237 & 14.70 \\
\hline 2nm & 1 & 1 & 36 & 1.12 & 4 & 0.07 & 232 & 14.39 \\
\hline 0nm & 1 & 1 & 33 & 1.33 & 90 & 1.62 & 286 & 17.73 \\
\hline Alert Bay 1 & Coastal & 1 & 39 & 1.21 & 10 & 0.18 & 241 & 14.94 \\
\hline Alert Bay 2 & Coastal & 1 & 38 & 1.18 & 16 & 0.29 & 239 & 14.82 \\
\hline Stack Bay 1 & Coastal & 1 & 33 & 1.02 & 34 & 0.61 & 126 & 7.81 \\
\hline Stack Bay 2 & Coastal & 1 & 32 & 0.99 & 9 & 0.16 & 122 & 7.56 \\
\hline South Islet & Coastal & 1 & 28 & 0.87 & 6 & 0.11 & 116 & 7.19 \\
\hline South Islet & Coastal & 1 & 39 & 1.21 & 44 & 0.79 & 158 & 9.80 \\
\hline $\begin{array}{l}\text { Anchorage } \\
\text { Bay rock } \\
\text { pool }\end{array}$ & Coastal & 1 & 64 & 1.98 & 92 & 1.66 & 134 & 8.31 \\
\hline $\begin{array}{l}\text { Anchorage } \\
\text { Bay }\end{array}$ & Coastal & 1 & 39 & 1.21 & 10 & 0.18 & 244 & 15.13 \\
\hline 2nm & 2 & 1 & 34 & 1.05 & 25 & 0.45 & 258 & 16.00 \\
\hline 4.5nm & 2 & 1 & 37 & 1.15 & 7 & 0.13 & 229 & 14.20 \\
\hline 7nm & 2 & 1 & 36 & 1.18 & 15 & 0.27 & 241 & 14.94 \\
\hline 9.5nm & 2 & 1 & 38 & 1.18 & 7 & 0.13 & 239 & 14.82 \\
\hline 12nm & 2 & 1 & 37 & 1.14 & 5 & 0.09 & 236 & 14.63 \\
\hline
\end{tabular}


Table 3-2 List of water nutrient concentrations collected from the Bounty Islands.

\begin{tabular}{|c|c|c|c|c|c|c|c|c|}
\hline Site & Transect & $\mathrm{n}$ & \multicolumn{2}{|c|}{ DRP } & \multicolumn{2}{|c|}{$\mathrm{NH}_{4}-\mathrm{N}$} & \multicolumn{2}{|c|}{$\mathrm{NO}_{3}-\mathrm{N}$} \\
\hline & & & $\mathrm{mg} \cdot \mathrm{m}^{-3}$ & $\mu \mathrm{m}$ & mg.m ${ }^{-3}$ & $\mu \mathrm{m}$ & mg.m ${ }^{-3}$ & $\mu \mathrm{m}$ \\
\hline $12 \mathrm{~nm}$ & 1 & 1 & 33 & 1.02 & 5 & 0.09 & 200 & 12.40 \\
\hline $9.5 \mathrm{~nm}$ & 1 & 1 & 33 & 1.02 & 3 & 0.05 & 211 & 13.08 \\
\hline $7 \mathrm{~nm}$ & 1 & 1 & 33 & 1.02 & 4 & 0.07 & 212 & 13.15 \\
\hline $4.5 \mathrm{~nm}$ & 1 & 1 & 31 & 0.96 & 7 & 0.13 & 195 & 12.09 \\
\hline $2 \mathrm{~nm}$ & 1 & 1 & 38 & 0.96 & 4 & 0.07 & 197 & 12.21 \\
\hline $\begin{array}{l}\text { Tunnel } \\
\text { Island }\end{array}$ & 1 & 1 & 30 & 1.18 & 18 & 0.32 & 226 & 14.01 \\
\hline $\begin{array}{l}\text { Lion } \\
\text { Island } 1\end{array}$ & Coastal & 1 & 30 & 0.93 & 7 & 0.13 & 219 & 13.58 \\
\hline $\begin{array}{l}\text { Lion } \\
\text { Island } 2\end{array}$ & Coastal & & 37 & 1.15 & 16 & 0.29 & 208 & 12.90 \\
\hline $\begin{array}{l}\text { Tunnel } \\
\text { Island } \\
\text { Channel }\end{array}$ & Coastal & & 37 & 1.15 & 25 & 0.45 & 222 & 13.77 \\
\hline $\begin{array}{l}\text { Molly } \\
\text { Cap } 1\end{array}$ & Coastal & & 34 & 1.05 & 28 & 0.51 & 195 & 12.09 \\
\hline $\begin{array}{l}\text { Molly } \\
\text { Cap } 2\end{array}$ & Coastal & & 30 & 0.93 & 6 & 0.11 & 204 & 12.65 \\
\hline $\begin{array}{l}\text { Funnel } \\
\text { Island } 1\end{array}$ & Coastal & & 34 & 1.05 & 5 & 0.09 & 206 & 12.77 \\
\hline $\begin{array}{l}\text { Funnel } \\
\text { Island } 2\end{array}$ & Coastal & & 38 & 1.18 & 4 & 0.07 & 202 & 12.53 \\
\hline $\begin{array}{l}\text { Depot } \\
\text { Island } \\
\text { west } 1\end{array}$ & Coastal & & 35 & 1.08 & 16 & 0.29 & 208 & 12.90 \\
\hline $\begin{array}{l}\text { Depot } \\
\text { Island } \\
\text { west } 2\end{array}$ & Coastal & & 34 & 1.05 & 23 & 0.41 & 209 & 12.96 \\
\hline $2 \mathrm{~nm}$ & 2 & & 30 & 0.93 & 11 & 0.20 & 183 & 11.35 \\
\hline $4.5 \mathrm{~nm}$ & 2 & & 29 & 0.90 & 7 & 0.13 & 174 & 10.79 \\
\hline $7 \mathrm{~nm}$ & 2 & & 28 & 0.87 & 5 & 0.09 & 176 & 10.91 \\
\hline $9.5 \mathrm{~nm}$ & 2 & & 29 & 0.90 & 23 & 0.41 & 172 & 10.66 \\
\hline $12 \mathrm{~nm}$ & 2 & & 30 & 0.93 & 8 & 0.14 & 175 & 10.85 \\
\hline
\end{tabular}


Table 3-3 List of water nutrient concentrations collected from the open ocean.

\begin{tabular}{|l|l|l|l|l|l|l|l|}
\hline Site & $\mathrm{N}$ & \multicolumn{3}{|c|}{$\mathrm{DRP}$} & \multicolumn{2}{c|}{$\mathrm{NH}_{4}-\mathrm{N}$} & \multicolumn{2}{c|}{$\mathrm{NO}_{3}-\mathrm{N}$} \\
\hline & & $\mathrm{mg} \cdot \mathrm{m}^{-3}$ & $\mu \mathrm{m}$ & $\mathrm{mg} \cdot \mathrm{m}^{-3}$ & $\mu \mathrm{m}$ & $\mathrm{mg} \cdot \mathrm{m}^{-3}$ & $\mu \mathrm{m}$ \\
\hline $\begin{array}{l}\text { Open } \\
\text { Ocean 1 }\end{array}$ & 1 & 53 & 1.64 & 5 & 0.09 & 219 & 03.58 \\
\hline $\begin{array}{l}\text { Open } \\
\text { Ocean 2 }\end{array}$ & 1 & 34 & 1.05 & 21 & 0.38 & 214 & 13.27 \\
\hline $\begin{array}{l}\text { Open } \\
\text { Ocean 3 }\end{array}$ & 1 & 37 & 1.15 & 15 & 0.27 & 237 & 14.70 \\
\hline $\begin{array}{l}\text { Open } \\
\text { Ocean 4 }\end{array}$ & 1 & 39 & 1.21 & 13 & 0.23 & 260 & 16.12 \\
\hline $\begin{array}{l}\text { Open } \\
\text { Ocean 5 }\end{array}$ & 1 & 31 & 0.96 & 25 & 0.45 & 202 & 12.53 \\
\hline $\begin{array}{l}\text { Open } \\
\text { Ocean 6 }\end{array}$ & 1 & 25 & 0.77 & 12 & 0.22 & 152 & 9.42 \\
\hline $\begin{array}{l}\text { Open } \\
\text { Ocean 7 }\end{array}$ & 1 & 29 & 0.90 & 10 & 0.18 & 171 & 10.60 \\
\hline
\end{tabular}


Table 3-4 List of phytoplankton stable isotope values collected from Antipodes Island.

\begin{tabular}{|l|l|l|l|l|}
\hline Site & Transect & $\mathrm{n}$ & $\delta^{13} \mathrm{C}$ & $\delta^{15} \mathrm{~N}$ \\
\hline & & & & \\
\hline $12 \mathrm{~nm}$ & 1 & 1 & -22.48 & -0.02 \\
\hline $9.5 \mathrm{~nm}$ & 1 & 1 & -22.65 & -1.03 \\
\hline $7 \mathrm{~nm}$ & 1 & 1 & -22.46 & -0.62 \\
\hline $4.5 \mathrm{~nm}$ & 1 & 1 & -22.52 & -2.19 \\
\hline $2 \mathrm{~nm}$ & 1 & 1 & -22.06 & -4.75 \\
\hline Alert Bay & Coastal & 1 & -23.06 & 1.87 \\
\hline Ringdove Bay & Coastal & 1 & -23.52 & 0.38 \\
\hline South Islet & Coastal & 1 & -22.16 & 0.64 \\
\hline $2 \mathrm{~nm}$ & 2 & 1 & -23.24 & 0.04 \\
\hline $4.5 \mathrm{~nm}$ & 2 & 1 & -23.07 & -0.41 \\
\hline $7 \mathrm{~nm}$ & 2 & 1 & -22.32 & -1.31 \\
\hline $9.5 \mathrm{~nm}$ & 2 & 1 & -23.31 & 1.70 \\
\hline $12 \mathrm{~nm}$ & 2 & 1 & -22.12 & -1.37 \\
\hline
\end{tabular}

Table 3-5 List of phytoplankton stable isotope values collected from the Bounty Islands.

\begin{tabular}{|l|l|l|l|l|}
\hline Site & Transect & $\mathrm{n}$ & $\delta^{13} \mathrm{C}$ & $\delta^{15} \mathrm{~N}$ \\
\hline & & & & \\
\hline $12 \mathrm{~nm}$ & 1 & 1 & -20.67 & 3.80 \\
\hline $9.5 \mathrm{~nm}$ & 1 & 1 & -20.35 & 2.36 \\
\hline $7 \mathrm{~nm}$ & 1 & 1 & -20.50 & 3.23 \\
\hline $4.5 \mathrm{~nm}$ & 1 & 1 & -20.34 & 1.19 \\
\hline $2 \mathrm{~nm}$ & 1 & 1 & -20.53 & 1.35 \\
\hline Tunnel Island & Coastal & 1 & -18.90 & 4.01 \\
\hline Depot Island west 2 & Coastal & 1 & -20.47 & 0.48 \\
\hline Funnel Island & Coastal & 1 & -22.56 & 4.13 \\
\hline $2 \mathrm{~nm}$ & 2 & 1 & -20.27 & 2.48 \\
\hline $4.5 \mathrm{~nm}$ & 2 & 1 & -20.48 & 2.96 \\
\hline $7 \mathrm{~nm}$ & 2 & 1 & -20.68 & 1.19 \\
\hline $9.5 \mathrm{~nm}$ & 2 & 1 & -20.49 & 1.57 \\
\hline $12 \mathrm{~nm}$ & 2 & 1 & -20.15 & 7.08 \\
\hline
\end{tabular}


Table 3-6 List of phytoplankton stable isotope values collected from the open ocean

\begin{tabular}{|l|l|l|l|}
\hline Site & $\mathrm{N}$ & $\delta^{13} \mathrm{C}$ & $\delta^{15} \mathrm{~N}$ \\
\hline $\begin{array}{l}\text { Open } \\
\text { Ocean 1 }\end{array}$ & 1 & -22.98 & -1.76 \\
\hline $\begin{array}{l}\text { Open } \\
\text { Ocean 2 }\end{array}$ & 1 & -24.47 & -0.78 \\
\hline $\begin{array}{l}\text { Open } \\
\text { Ocean 3 }\end{array}$ & 0 & & \\
\hline $\begin{array}{l}\text { Open } \\
\text { Ocean 4 }\end{array}$ & 0 & -19.34 & 0.94 \\
\hline $\begin{array}{l}\text { Open } \\
\text { Ocean 5 }\end{array}$ & 1 & -19.67 & 0.12 \\
\hline $\begin{array}{l}\text { Open } \\
\text { Ocean 6 }\end{array}$ & 1 & -21.83 & 0.38 \\
\hline $\begin{array}{l}\text { Open } \\
\text { Ocean 7 }\end{array}$ & 1 & & \\
\hline
\end{tabular}

Table 3-7 Correlation between distance and nutrient concentrations and phytoplankton stable isotopes (Pearson correlation coefficients) at the Antipodes and Bounty Islands

\begin{tabular}{|l|l|l|l|l|l|}
\hline Island & DRP & NH $4-\mathrm{N}$ & $\mathrm{NO}_{3}-\mathrm{N}$ & $\begin{array}{l}\text { Phytoplankton } \\
\text { Carbon } \\
\text { Isotopes }\end{array}$ & $\begin{array}{l}\text { Phytoplankton } \\
\text { Nitrogen } \\
\text { Isotopes }\end{array}$ \\
\hline $\begin{array}{l}\text { Antipodes } \\
\text { Island }\end{array}$ & $\begin{array}{l}\text { No } \\
\text { correlation } \\
\mathrm{p}>0.05\end{array}$ & $\begin{array}{l}\text { No } \\
\text { correlation } \\
\mathrm{p}>0.05\end{array}$ & $\begin{array}{l}\text { Positive } \\
\text { correlation } \\
.512 \\
\mathrm{p}<0.05\end{array}$ & $\begin{array}{l}\text { No correlation } \\
\mathrm{p}>0.05\end{array}$ & $\begin{array}{l}\text { No correlation } \\
\mathrm{p}>0.05\end{array}$ \\
\hline $\begin{array}{l}\text { Bounty } \\
\text { Islands }\end{array}$ & $\begin{array}{l}\text { Negative } \\
\text { correlation }\end{array}$ & $\begin{array}{l}\text { No } \\
\text { correlation } \\
\mathrm{p}>0.05\end{array}$ & $\begin{array}{l}\text { Negative } \\
\text { correlation } \\
-515\end{array}$ & $\begin{array}{l}\text { No correlation } \\
\mathrm{p}>0.05\end{array}$ & $\begin{array}{l}\text { No correlation } \\
\mathrm{p}>0.05\end{array}$ \\
$\mathrm{p}<0.05$
\end{tabular}


Table 3-8 Fractions of kelp, phytoplankton (phytopl.) and terrestrial carbon in consumers at the Antipodes Island

\begin{tabular}{|c|c|c|c|c|c|}
\hline Sample & $\mathrm{n}$ & $\begin{array}{l}\text { Average } \delta^{13} \mathrm{C} \\
\text { and SD }\end{array}$ & \% Kelp & $\begin{array}{l}\% \\
\text { Phytopl. }\end{array}$ & $\begin{array}{l}\% \\
\text { Terrestrial }\end{array}$ \\
\hline $\begin{array}{l}\text { Zooplankton } \\
\text { Euphausiid }\end{array}$ & 1 & -21.29 & 56 & 44 & \\
\hline $\begin{array}{l}\text { Porifera } \\
\text { Darwinella gardineri } \\
\text { Latrunculia brevis }\end{array}$ & $\begin{array}{l}1 \\
1\end{array}$ & $\begin{array}{l}-21.60 \\
-19.11\end{array}$ & $\begin{array}{l}37 \\
100\end{array}$ & 63 & \\
\hline $\begin{array}{l}\text { Cnidaria } \\
\text { Hydroid } \\
\text { Anenomes }\end{array}$ & $\begin{array}{l}2 \\
3\end{array}$ & $\begin{array}{l}-21.72 \pm 0.16 \\
-20.60 \pm 0.07\end{array}$ & $\begin{array}{l}30 \\
99\end{array}$ & $\begin{array}{l}70 \\
1\end{array}$ & \\
\hline $\begin{array}{l}\text { Crustacea } \\
\text { Leptomithrax australis } \\
\text { Tubeworms } \\
\text { Balanus }\end{array}$ & $\begin{array}{l}1 \\
3 \\
1\end{array}$ & $\begin{array}{l}-18.48 \\
-20.75 \pm 0.46 \\
-21.72\end{array}$ & $\begin{array}{l}100 \\
90 \\
29\end{array}$ & $\begin{array}{l}10 \\
71\end{array}$ & \\
\hline $\begin{array}{l}\text { Mollusca } \\
\text { Eudoxochiton nobilis } \\
\text { Aulacomya moaoriana } \\
\text { Buccinulum pertinax } \\
\text { Cellana strigilis } \\
\text { Haliotis virgínea huttoni } \\
\text { Calliostoma eminens } \\
\text { Fusitriton laudandus }\end{array}$ & $\begin{array}{l}4 \\
3 \\
3 \\
4 \\
3 \\
3 \\
3\end{array}$ & $\begin{array}{l}-22.71 \pm 5.13 \\
-21.43 \pm 0.46 \\
-20.60 \pm 0.08 \\
-12.72 \pm 2.48 \\
-23.92 \pm 0.69 \\
-19.31 \pm 0.32 \\
-19.44 \pm 0.39\end{array}$ & $\begin{array}{l}47 \\
99 \\
100 \\
100 \\
100\end{array}$ & $\begin{array}{l}100 \\
53 \\
1 \\
100\end{array}$ & \\
\hline $\begin{array}{l}\text { Bryozoa } \\
\text { Bryozoans }\end{array}$ & 4 & $-4.25 \pm 1.49$ & & 100 & \\
\hline $\begin{array}{l}\text { Echinodermata } \\
\text { Henricia sp } \\
\text { Paranepanthia aucklandensis } \\
\text { Ophiomyxa brevirima } \\
\text { Squamocnus brevidentis }\end{array}$ & $\begin{array}{l}3 \\
4 \\
2 \\
2\end{array}$ & $\begin{array}{l}-20.31 \pm 0.39 \\
-17.43 \pm 1.29 \\
-4.31 \pm 1.20 \\
-17.65 \pm 1.41\end{array}$ & $\begin{array}{l}100 \\
100 \\
100 \\
100\end{array}$ & & \\
\hline $\begin{array}{l}\text { Chordata } \\
\text { Tunicata } \\
\text { Salp } \\
\text { Fish } \\
\text { Bovichtus variegatus }\end{array}$ & 1 & $\begin{array}{l}-22.08 \\
-19.49\end{array}$ & $\begin{array}{l}7 \\
100\end{array}$ & 93 & \\
\hline $\begin{array}{l}\text { Terrestrial birds } \\
\text { Cyanoramphus unicolor } \\
\text { C. novaezelandiae hochstetteri } \\
\text { Snipe }\end{array}$ & $\begin{array}{l}3 \\
2 \\
2\end{array}$ & $\begin{array}{l}-23.23 \pm 1.89 \\
-24.01 \pm 1.89 \\
-22.45 \pm 0.38\end{array}$ & & $\begin{array}{l}49 \\
86 \\
12\end{array}$ & $\begin{array}{l}51 \\
14 \\
88\end{array}$ \\
\hline $\begin{array}{l}\text { Terrestrial Invertebrates } \\
\text { worms } \\
\text { Flies } \\
\text { Fleas } \\
\text { Ticks }\end{array}$ & $\begin{array}{l}1 \\
3 \\
2 \\
1\end{array}$ & $\begin{array}{l}-14.07 \\
-21.86 \pm 0.37 \\
-20.86 \pm 0.44 \\
-17.39\end{array}$ & $\begin{array}{l}21 \\
83\end{array}$ & $\begin{array}{l}79 \\
17 \\
100\end{array}$ & 100 \\
\hline $\begin{array}{l}\text { Terrestrial mammal } \\
\text { Mus musculus }\end{array}$ & 3 & $-18.93 \pm 0.65$ & & 21 & 79 \\
\hline
\end{tabular}


Table 3-9 Fractions of kelp and phytoplankton in consumers' diets at the Bounty Islands

\begin{tabular}{|c|c|c|c|c|}
\hline Sample & $n$ & $\begin{array}{l}\text { Average } \delta^{13} \mathrm{C} \\
\text { and SD }\end{array}$ & \% Kelp & \% Phytoplankton \\
\hline $\begin{array}{l}\text { Zooplankton } \\
\text { Euphausiid }\end{array}$ & 1 & -20.59 & 4 & 96 \\
\hline $\begin{array}{l}\text { Porifera } \\
\text { Hymeniacidon indistincta } \\
\text { Callyspongia sp.nov } 12 \\
\text { sponge }\end{array}$ & $\begin{array}{l}3 \\
3 \\
3\end{array}$ & $\begin{array}{l}-17.18 \pm 1.05 \\
-19.67 \pm 0.57 \\
-18.73 \pm 0.17\end{array}$ & & $\begin{array}{l}100 \\
100 \\
100\end{array}$ \\
\hline $\begin{array}{l}\text { Cnidaria } \\
\text { Anenomes }\end{array}$ & 3 & $-18.38 \pm 0.55$ & & 100 \\
\hline $\begin{array}{l}\text { Crustacea } \\
\text { Leptomithrax australis } \\
\text { Balanus } \\
\text { Shrimps } \\
\text { Talorchestia }\end{array}$ & $\begin{array}{l}3 \\
3 \\
1 \\
4\end{array}$ & $\begin{array}{l}-18.84 \pm 0.35 \\
-19.02 \pm 0.36 \\
-18.62 \\
-18.48 \pm 1.49\end{array}$ & & $\begin{array}{l}100 \\
100 \\
100 \\
100\end{array}$ \\
\hline $\begin{array}{l}\text { Mollusca } \\
\text { Aulacomya maoriana } \\
\text { Mytilus galloprovincialis } \\
\text { Cellana strigilis } \\
\text { Haliotis virginea huttoni } \\
\text { Janolus ignis } \\
\text { Haustrum lacunosum } \\
\text { Kerguelenella interalis } \\
\text { Onithochiton neglectus }\end{array}$ & $\begin{array}{l}6 \\
3 \\
10 \\
3 \\
1 \\
3 \\
3 \\
6\end{array}$ & $\begin{array}{l}-18.95 \pm 0.36 \\
-18.95 \pm 0.12 \\
-16.81 \pm 4.86 \\
-22.56 \pm 0.33 \\
-17.39 \\
-17.85 \pm 0.55 \\
-14.36 \pm 0.82 \\
-15.36 \pm 2.51\end{array}$ & 35 & $\begin{array}{l}100 \\
100 \\
100 \\
65 \\
100 \\
100 \\
100 \\
100\end{array}$ \\
\hline $\begin{array}{l}\text { Bryozoa } \\
\text { Bryozoan } \\
\text { Orthoscuticella ventricosa }\end{array}$ & $\begin{array}{l}10 \\
3\end{array}$ & $\begin{array}{l}-13.32 \pm 6.87 \\
-6.38 \pm 0.57\end{array}$ & & $\begin{array}{l}100 \\
100\end{array}$ \\
\hline $\begin{array}{l}\text { Echinodermata } \\
\text { Henricia sp } \\
\text { Starfish }\end{array}$ & $\begin{array}{l}3 \\
6\end{array}$ & $\begin{array}{l}-16.74 \pm 1.25 \\
-16.61 \pm 0.48\end{array}$ & & $\begin{array}{l}100 \\
100\end{array}$ \\
\hline $\begin{array}{l}\text { Terrestrial Invertebrates } \\
\text { Mosquitoes } \\
\text { Pacificana }\end{array}$ & $\begin{array}{l}3 \\
3\end{array}$ & $\begin{array}{l}-18.78 \pm 0.50 \\
-18.11 \pm 0.14\end{array}$ & & $\begin{array}{l}100 \\
100\end{array}$ \\
\hline
\end{tabular}


Figure 3-4 Water nutrient concentrations recorded on transects and at coastal sites around Antipodes Island.

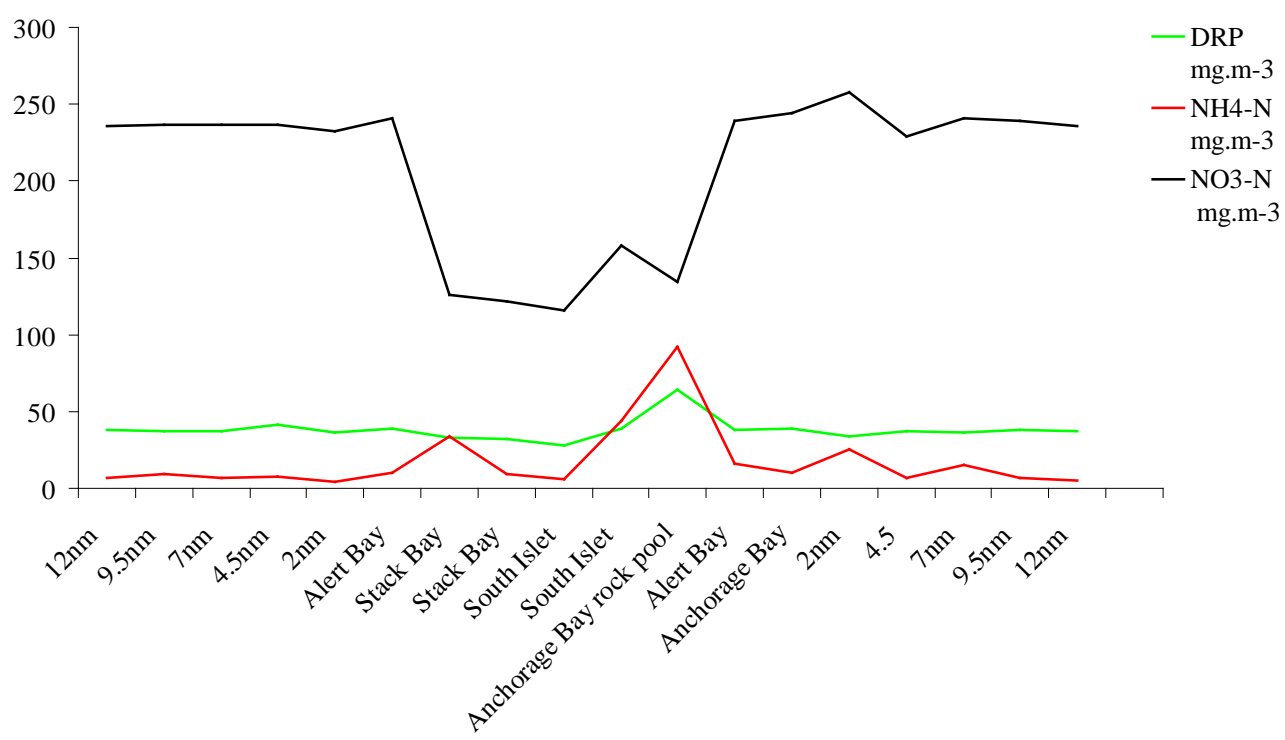

Figure 3-5 Water nutrient concentrations recorded on transects and at coastal sites around the Bounty Islands.

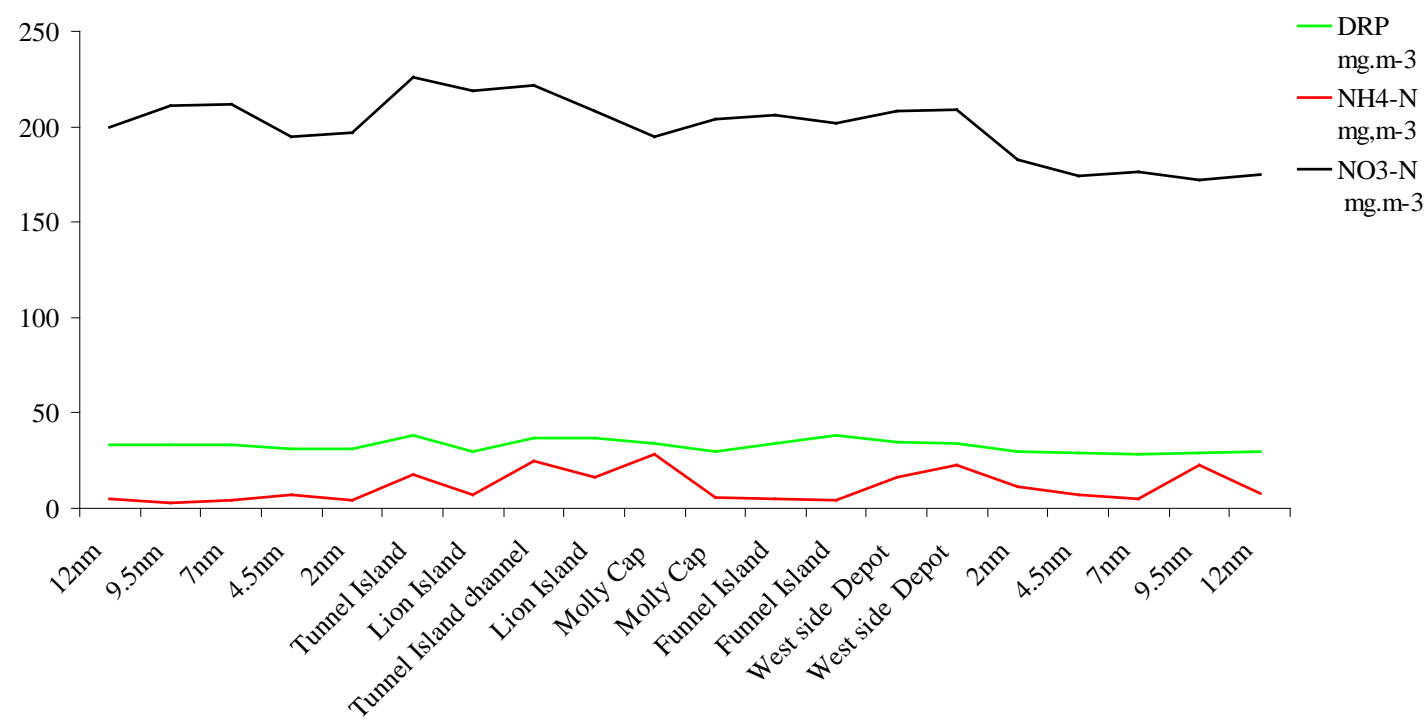


Figure 3-6 Water nutrient concentrations recorded at open ocean sites.

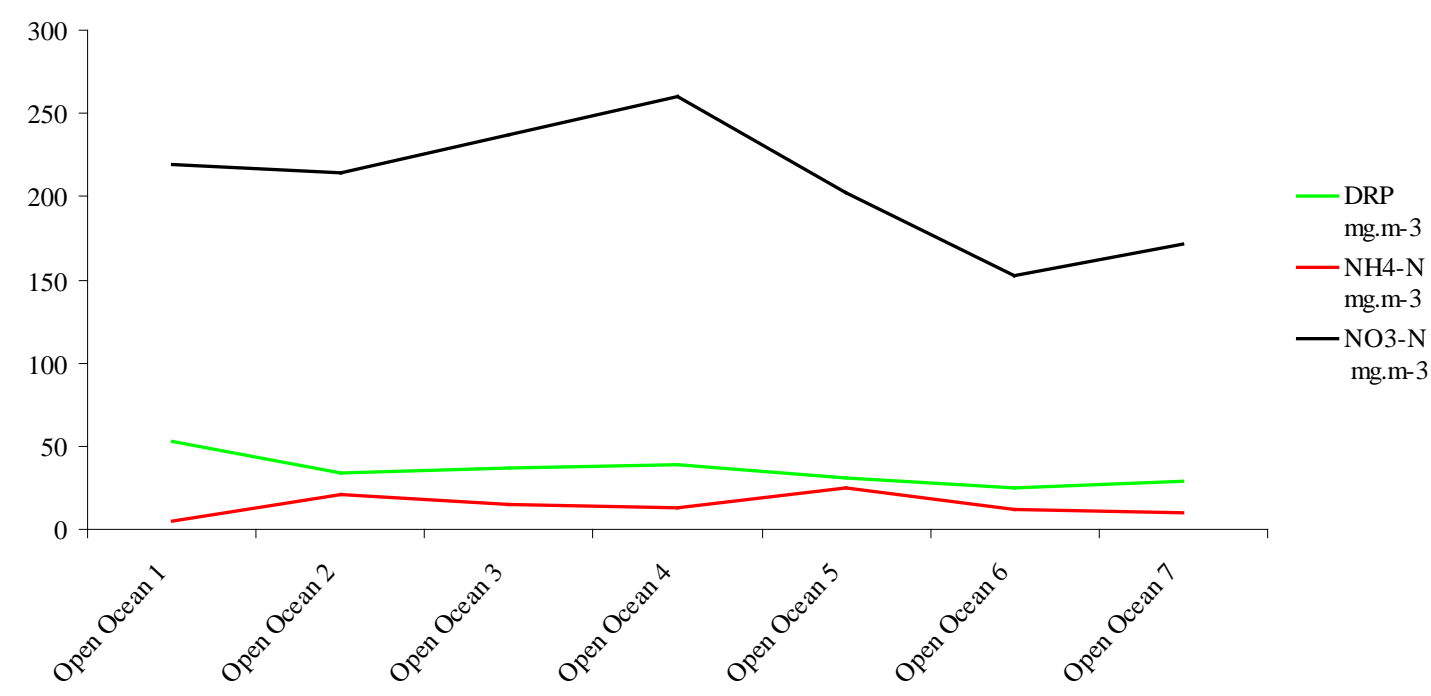


Figure 3-7 Phytoplankton stable isotope transect at Antipodes Island

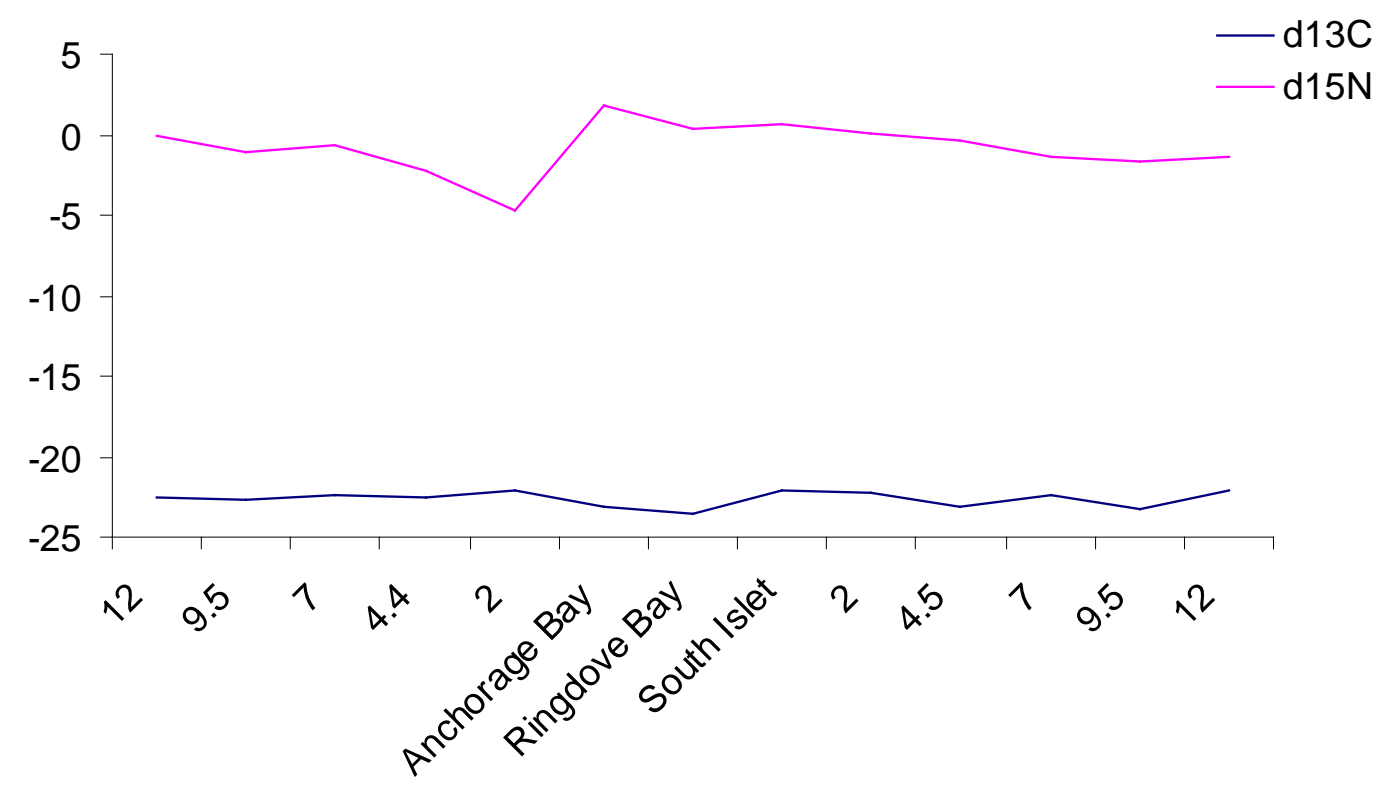

Figure 3-8 Phytoplankton stable isotope transect at the Bounty Islands

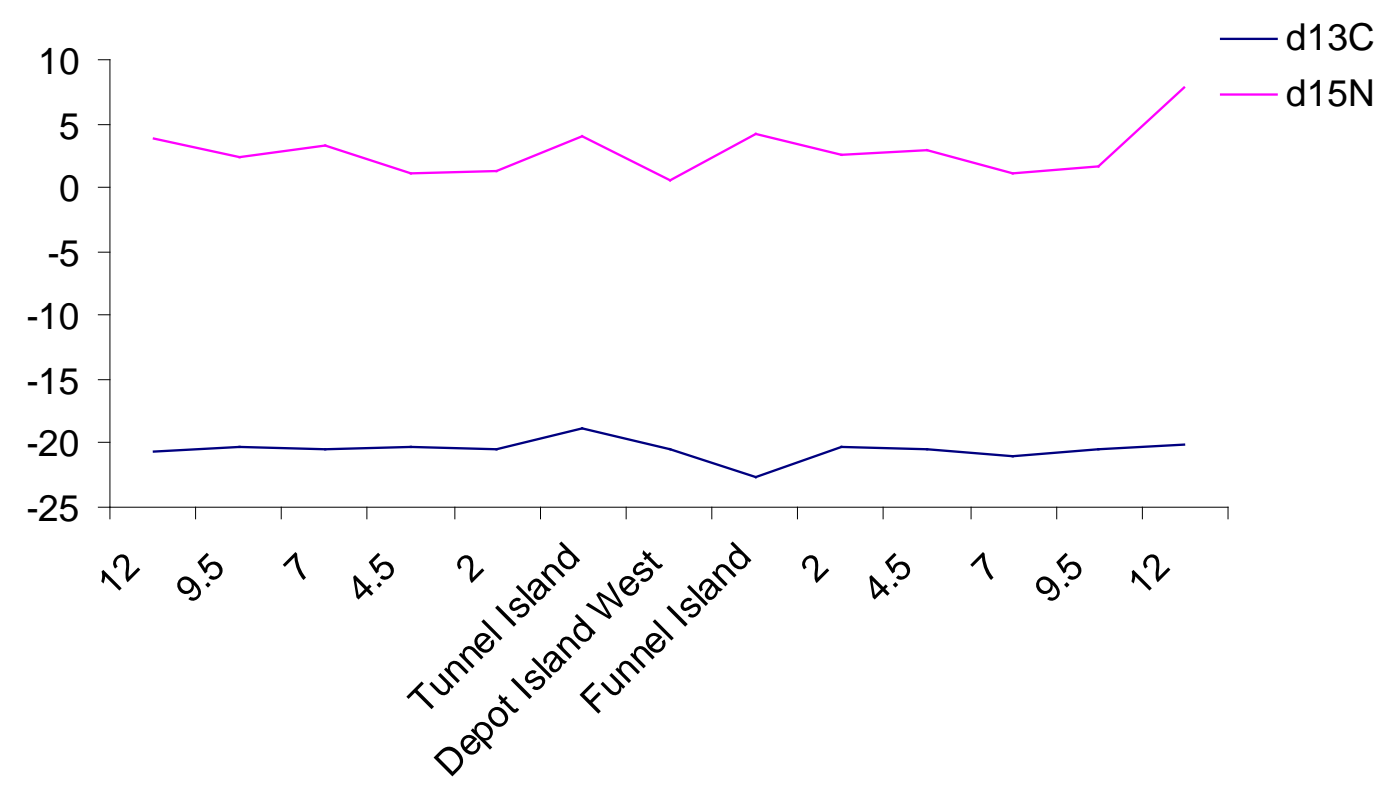


Figure 3-9 Phytoplankton stable isotope signatures from open ocean sites.

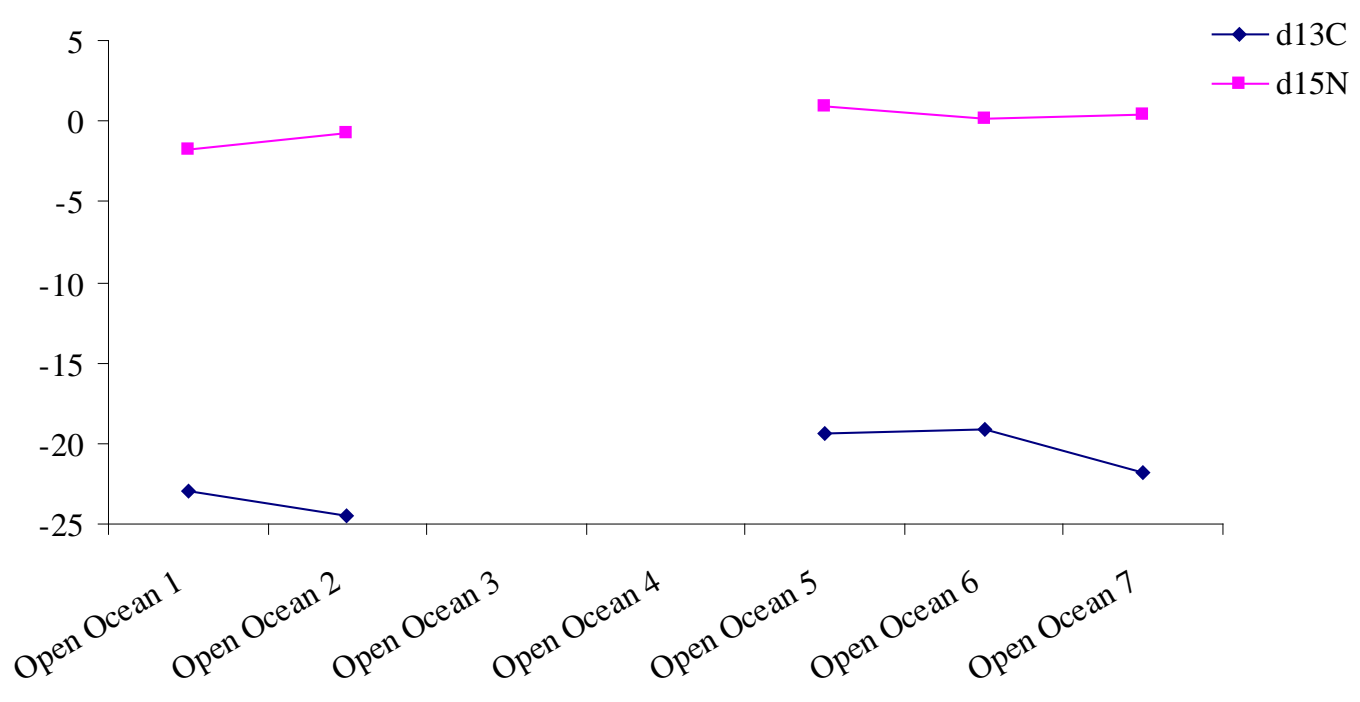




\subsection{DISCUSSION}

Information on physical and biological drivers is important for better understanding the structure and functioning of marine ecosystems. This study has provided important insights into these aspects at Antipodes and the Bounty Islands. In this Chapter I have described the levels of nutrients in the water surrounding both islands and shown that there is a spatial gradient at both islands, with nitrate levels showing a relationship with distance from shore. I have shown that macroalgae are an important component in the diets of consumers at Antipodes Island, but are less important at the Bounty Islands and that terrestrial species at both islands are utilising marine derived nutrients in their diets.

\subsubsection{WATER NUTRIENT CONCENTRATIONS}

Surface water dissolved organic nutrients sampled in this study (DRP, $\mathrm{NO}_{3}-\mathrm{N}$ and $\mathrm{NH}_{4}-\mathrm{N}$ ) were recorded at levels within the range reported by previous studies within subantarctic waters (SAW) and the South-west Pacific (BradfordGrieve et al. 1997). To my knowledge, the data collected during my study are the first water nutrient samples collected within $12 \mathrm{~nm}$ of the islands and from coastal sites on the islands themselves. My study shows that the region and the waters surrounding both Antipodes Island and the Bounty Islands in Autumn have nutrient concentration levels that are high in nitrate and phosphorous. The concentrations of nitrate and phosphorous in my study are consistent with values reported across the global 45-60 degree latitudes (Levitus et al., 1993, Rajakumar et al., 2008, Chang and Gall, 1998).

No significant difference was found in nutrient concentrations between the open ocean sites at Antipodes Island and the Bounty Islands, presumably as all sampling was undertaken within the boundary of Subantarctic Water (SAW) in autumn-winter. Chiswell et al. (2013) suggest that SAW is deeply mixed in the winter months and phytoplankton production is low due to low light and iron 
levels, resulting in less uptake of surface nutrients by phytoplankton. This suggests that variation in the concentration of water nutrients across my open ocean sites may be explained by patchy elevation in chlorophyll a across the Campbell Plateau as previously described by Heath and Bradford (1980). These authors also proposed that conditions required for large scale phytoplankton blooms do not exist in this region due to the lack of iron and light. This may result in overall lack of nutrient uptake and therefore little variability of nutrients concentrations across SAW. Low chlorophyll a values across SAW are interrupted by localised plankton accumulations around the Subantarctic Islands (Murphy et al., 2001). Nutrient concentrations collected during my study showed positive and negative correlations between their values and distance from shore. These correlations may suggest that there are particular processes occurring that are associated with the islands.

There was no significant difference in phytoplankton carbon and nitrogen isotope values between Antipodes Island and the Bounty Islands, but open ocean sites did differ significantly from samples taken from within the $12 \mathrm{~nm}$ at both islands. There is a gradient that ranges from high carbon values in subtropical areas to low carbon values in Antarctic waters (Francois et al., 1993, Trull and Armand, 2001) but I found no clear evidence for this in my study. It is possible that there was not a large enough latitudinal distance in this study to detect any such gradient. Nitrogen stable isotope values may have differed between the island sites and open ocean due to the nitrogen enrichment that can be attributed due to the input of nutrients from seabirds. For example, Wainright et al. (1998) found phytoplankton around seabird colonies in the Arctic region round St Paul were enriched in nitrogen compared to those sites with no sea birds. Similarly, McCauley et al. (2012) found enhanced nutrients and plankton productivity around a Pacific atoll that supported seabirds.

\subsubsection{WATER NUTRIENT AND STABLE ISOTOPE TRANSECTS}

Water nutrient samples taken every $2.5 \mathrm{~nm}$ from within the $12 \mathrm{~nm}$ mark from both islands showed a correlation between distance from shore and nutrient 
concentrations for $\mathrm{NO}_{3}-\mathrm{N}$ at both of the islands and DRP at the Bounty Islands. No trend was found between distance from the islands and concentrations of $\mathrm{NO}_{4}-\mathrm{N}$ at either island. Nutrient concentrations at Antipodes Island past the $2.5 \mathrm{~nm}$ station showed little variability and were similar to those of the open ocean sites on both transects. $\mathrm{NO}_{3}-\mathrm{N}$ showed a significant negative correlation, decreasing by up to $50 \%$ compared to sites further offshore, suggesting that $\mathrm{NO}_{3}$ $\mathrm{N}$ may be an important nutrient that is being taken up by nearshore phytoplankton. In the most recent study of chlorophyll a in the southwest Pacific Ocean, Chiswell et al. (2013) suggested that while there are localised phytoplankton blooms around other Subantarctic Islands there is no clear pattern for elevated production at Antipodes Island due to its position on the shelf edge. The low phytoplankton production would suggest that the uptake of $\mathrm{NO}_{3}-\mathrm{N}$ by phytoplankton would not lead to such significant decreases of $\mathrm{NO}_{3}-\mathrm{N}$ concentration around the nearshore environment. Satellite imagery of chlorophyll a concentrations observed from Moderate Resolution Imaging Spectroradiometers (MODIS, http://oceancolor.gsfc.nasa.gov/) also showed very low chlorophyll a pigment for Antipodes Island in April 2009 (when I undertook my sampling), indicating that there was very low primary production occurring at the island (Figure 3-10).

I hypothesise that if phytoplankton is not responsible for the utilisation of $\mathrm{NO}_{3}$ $\mathrm{N}$, that macroalgae might play a significant role in the uptake of $\mathrm{NO}_{3}-\mathrm{N}$ at Antipodes Island. There was little variability in $\mathrm{NO}_{4}-\mathrm{N}$ around the island, with similar values to open ocean sites and this nutrient occurred at concentrations above that required to limit $\mathrm{NO}_{3}-\mathrm{N}$ uptake by macroalgae (Thomas and Harrison, 1987). Antipodes Island has large bed-forming macroalgae (Nelson, 1994) that surround the majority of the island and that would require sufficient nutrients to maintain growth, particularly when light is most available. It is well known that macroalgae productivity is regulated by light and nutrients and when light levels are low there is often high nutrient levels in the water with little utilisation by macroalgae (Gagné et al., 1982). However, Phillips and Hurd (2003) showed that in winter, in areas south of Dunedin, New Zealand, when higher concentrations of $\mathrm{NO}_{3}-\mathrm{N}$ were present, $\mathrm{NO}_{3}-\mathrm{N}$ was the most important nutrient taken up by intertidal macroalgae. Whilst my study did not attempt to determine uptake rates 
by macroalgae at Antipodes Island, the high concentrations of $\mathrm{NO}_{3}-\mathrm{N}$ year round would make it possible for high growth rates when other conditions (e.g. water temperature and light) are conducive to algal growth and this may have an impact on overall nitrogen budgets close to shore.

Similar to Antipodes Island, the Bounty Islands showed no trend in regards to $\mathrm{NO}_{4}-\mathrm{N}$ along the two transects. In contrast to Antipodes, the Bounty Islands showed a trend for $\mathrm{NO}_{3}-\mathrm{N}$ and DRP to increase closer to shore. This nutrient gradient may be explained by the continuous input of excrement that flows from the islands, originating from the substantial numbers of seabirds and marine mammals, reintroducing $\mathrm{NO}_{3}-\mathrm{N}$ and phosphates into the nearshore marine environment, minimising nutrient loss through primary production of phytoplankton. Kolb et al. (2010) showed nesting cormorants in the Stockholm archipelago in the northern Baltic Sea created high nutrient loading in runoff from the islands into the surrounding waters. They suggested this affects marine communities in similar ways to marine fertilization experiments, by concentrating nutrients and enhancing localised primary production. The reintroduction of fertilising nitrogen and phosphorous to waters surrounding islands may play an important role in enhancing local primary production (Golovkin, 1967).

\title{
3.4.3 CONTRIBUTION OF KELP, PHYTOPLANKTON AND TERRESTRIAL CARBON TO CONSUMERS' DIETS
}

\begin{abstract}
A basic mixing model was used where the single isotope of carbon from consumers' tissue was measured to determine the percentage contribution of kelp-derived carbon and phytoplankton-derived carbon, or phytoplankton- and terrestrial-derived carbon, to the diet of the consumers based on isotopic mass balance. Antipodes Island benthic consumers assimilate a considerable amount of kelp carbon in their diets but also phytoplankton-derived carbon, indicating that the Antipodes Island invertebrates demonstrate varied feeding patterns. Suspension feeders were the most varied in their fractions of kelp carbon, demonstrating that they use both kelp detritus and plankton as a food source. The
\end{abstract}


grazers Cellana strigilis, Haliotis virginea huttoni and chitons contrasted in their use of kelp, with Haliotis virginea huttoni and chitons utilising no kelp and Cellana strigilis all kelp in their diet. Haliotis species are known to graze on diatoms (Roberts et al., 1999). Benthic diatom grazing could also explain the large component of phytoplankton in the chiton diet as they were observed grazing on the surface of coralline algae where diatom films may be present. Limpets showed a high level of kelp in their diets. Limpets are known to be generalist grazers but also are able to exclusively feed on macroalgae (Creese, 1988) and have been recorded as having kelp based diets consisting of algal spores and Durvillaea antarctica at Marion Island (Blankley and Branch, 1985). Predatory gastropods also showed a strong influence of kelp in their diets which could indicate predation on other gastropods such as Buccinulum pertinax that displayed a high dependence on kelp in their diet. Echinoderms had a high kelp component to their diet which also suggests that these opportunistic feeders may be feeding on species that have high kelp based diets.

My results are consistent with other studies that have documented the important role of kelp in some marine communities (Dunton and Schell, 1987, Eckman et al., 1989, Bustamante and Branch, 1996) and in particular subantarctic islands (Kaehler et al., 2006). Interestingly the results presented here are very similar to those for the Antarctic Peninsula, where Dunton (2001) found very high kelp assimilation in limpets, echinoderms, deposit deeding bivalves and gastropods and concluded that macroalgae provides not only an important habitat but an important carbon source on the Antarctic Peninsula.

All terrestrial bird species at Antipodes Island had both a marine and terrestrial carbon presence in their diets, suggesting a strong marine-terrestrial linkage at the islands. The Antipodes parakeet Cyanoramphus unicolor has been observed feeding on a range of diet items including tussock, sedges, seeds, flowers, dead and predated seabirds, insects in beach drift algae and foraging in guano at the islands (Greene, 1995). My results are consistent with the limited diet studies on these birds by Greene (1995) who also recorded a diet of marine based and terrestrial based food. I have also observed red-crowned parakeets foraging in penguin guano at Antipodes Island (Figure 3-11). Snipe are known to feed on 
predominately terrestrial insect species but have been observed foraging on isopods at Antipodes Island (Higgins, 1996).

Mus musculus, the common house mouse and the only introduced pest at Antipodes Island, also show a combination of both marine and terrestrial carbon which indicates that they could be predating or scavenging on marine birds. It has been assumed that mice scavenge on marine species at the Antipodes but there have been no quantitative studies on the diet of mice at Antipodes Island. Stable isotope research on the diet of Keens's mice on seabird colonies in remote British Colombia by Drever et al. (2000) found that mice feed on the eggs or carcasses of chicks and adults, throughout the breeding season. Cuthbert and Hilton (2004) also observed a similar pattern of predation on seabird colonies on Gough Island, central South Atlantic Ocean. Fleas, ticks and flies showed a mix of kelp and phytoplankton-derived carbon in their diets, with flies and ticks being dependent on phytoplankton, which would be consistent with flies feeding on the carrion or excrement from seabirds and marine mammals (Polis et al., 2004) and with parasitic ticks being found in seabird burrows and on the bodies of the birds (Murray and Vestjens, 1967).

I found different results at the Bounty Islands compared to Antipodes Island. With the exception of Haliotis virginea huttoni, all species at the Bounty Islands showed high dependency on phytoplankton-derived carbon. This result is not consistent with studies that found kelp to be an important part of consumer diets at other Subantarctic islands (Kaehler et al., 2006) but is consistent with a study of Bouvet Island that found phytoplankton to be the most important carbon source (Jacob et al. 2006). This might indicate that large brown macroalgae is not a major carbon source for species at the Bounty Islands but other smaller forms of macroalgae or microalgae could be important. It could also be possible that the Bounty Islands has a more steady supply of phytoplankton than Antipodes Island. Freeman et al. (2011) reported that the Bounty Islands benthic communities were dominated by sessile filter feeding invertebrates (sponges, bivalves, bryozoans, barnacles) and that beds of large brown algae were absent or patchy at many of the sites surveyed, but some rock walls did support smaller forms of brown and red algae. They also recorded that grazers were most 
commonly found on isolated patches of coralline algae with no encrusting or macroalgae species present, supporting my finding that kelp is not an important contributor of carbon at the Bounty Islands.

\subsubsection{ISOSOURCE CASE STUDIES}

The introduction of additional diet sources when using Isosource displayed some consistent results with the two-source model but also some conflicting results. For example, for echinoderms, the basic mixing model suggested kelp comprised $100 \%$ of their diet but Isosource suggested they were feeding on a range of species including bryozoans and sponges that are known to feed on phytoplankton (Gili and Coma, 1998).

While there were some inconsistencies between the two models, they both demonstrated that Antipodes Island invertebrate consumers feed on a wide range of species with similar signatures to macroalgae, sponges, bryozoans, molluscs and gastropods. These models support the suggestion in Chapter Two that opportunistic feeding patterns exist where invertebrate consumers are predating or scavenging on species that have both macroalgae and phytoplankton in their diets. The presence of sponge sand bryozoans in the diet of the echinoderms may suggest that they are feeding on species whose diets consist of phytoplankton or benthic diatoms - this could be the case with Squamocnus brevidentis and Haliotis virginea huttoni, which had a similar isotope signature to sponges and bryozoans. An alternative explanation could be that echinoderms are using bryozoans and sponges as a dietary component. In a review by McClintock (1994) on Antarctic and subantarctic echinoderms, bryozoans and sponges were a feature of asteroid and holothuroid diets. A review on predation on bryozoan colonies by Lingard (2008) reported that the overall consumption of bryozoans may be under-represented. Lingard suggested that of the 286 studies of species diets, a high number of both mollusc and echinoderm species had bryozoans represented in their diets, which suggest that my modelling results may reflect the actual diet of echinoderms. 
The diet of the most abundant mobile consumers at the Bounty Islands followed a similar pattern to Antipodes Island, with a range of food items. Macroalgae, in particular red algae, appeared to be more important as a diet component than larger bed forming macroalgae which may provide some explanation for the lack of kelp represented in diets based of the kelp/phytoplankton model. Bryozoans, molluscs and gastropods were also a common diet item of the species sampled. The run off sample collected from one of the faecal /detritus streams was also present in the diets of the grazers and shrimps. Given the high densities of marine mammals and birds reported at the islands (Booth, 2004) it is possible that the detrital matter entering the near shore may be contributing to some opportunistic scavengers and grazers diets either by direct utilisation or secondary consumption through grazing. In a study on small islands in the Gulf of California, Polis and Hurd (1996) found that carrion supported a diverse set of detritivorus and scavenging crustaceans and insects in the intertidal and subtidal zones.

The diet of terrestrial birds at Antipodes Island determined using Isosource, was consistent with the 2-source mixing model showing that the diets of the endemic parakeets Cyanoramphus unicolor and Cyanoramphus hochstetteri consisted of both marine and terrestrial components. The results also suggested that these birds are consuming the introduced mice. This is important, as any future attempt to control mice on the islands needs to take this into consideration, for example if poison baits are to be used. The Antipodes Island snipe's diet was mainly from terrestrial based sources, with plant material being the major component of its diet. The diet of mice Mus musculus at Antipodes Island showed that they are scavenging on a wide range of species including birds and plant material. This new information confirms mice on the islands do utilise bird species in their diet. Mice may be having a negative impact on the food resources for native terrestrial species and this strengthens the case for removing mice from the islands.

The Bounty Islands terrestrial endemic spider Pacificana had a diet that consisted of other Pacificana, i.e. there is cannibalism or sexual cannibalism in the population, a relatively common feature of spiders (Wise, 2006). All the spiders that were collected in this study were female spiders, which may support 
this conclusion. This spider species does not build webs but lives in burrows constructed from fur seal fur (Figure 3-12) which may indicate that they are a hunting spider (Denno et al., 2004). A number of stable isotope studies have been undertaken to determine the influence of marine based nutrients on terrestrial systems, in particular on the role seabird guano plays in enriching the soil around nesting colonies (Ellis et al., 2006). Ellis et al., (2006) found that all the plants that were adjacent to gull or cormorant colonies in their study sites showed enriched $\mathrm{N}$ signatures that indicated the input of marine derived nutrients. Similarly, Anderson and Polis (1998) found that on islands in the Gulf of California, terrestrial species (spiders and scorpions) that inhabited the islands had more enriched $\mathrm{C}$ and $\mathrm{N}$ than inland species, suggesting that these species consumed more marine based food than inland species. Sánchez-Piñero and Polis (2000) found that the population of beetles within seabird nesting colonies in the Gulf of California were five times higher than those outside the colony and that not only the plant life on the islands benefitted from the nutrient input of seabirds but the island ecosystem was controlled by the density of the nesting birds, suggesting that on small islands the system is ultimately dependant on the subsidies from the bottom up - a donor controlled ecosystem. In a similar investigation, Barrett et al. (2005) found that the diet of lizards in the coastal zone consisted of $40 \%$ arthropods that were consuming microalgae and had substantially elevated trophic levels to those found inland. Such important interactions appear to be present in the New Zealand Subantarctic Islands I studied.

\subsubsection{CONCLUSIONS}

In summary, I showed that the drivers of the Antipodes and Bounty Islands ecosystems are different. I found that Antipodes Island is influenced by kelp and the Bounty Islands by phytoplankton but that both islands are influenced by nutrient subsidies from seabirds and marine mammals, demonstrating strong marine terrestrial linkages. I determined that there were relationships between nutrient concentrations and distance from shore at each island and that differences occurred in nutrient concentrations between Antipodes and the 
Bounty Islands. I was able to use stable isotope signatures to determine the contribution of phytoplankton, kelp and terrestrial food sources to the species of both islands. My results demonstrate strong linkages between the marine environment and terrestrial species at both Antipodes and the Bounty Islands and suggests that nutrients from species that cross ecosystem and habitat boundaries such as marine birds and marine mammals play an important role in the structure and functioning of the Antipodes and Bounty Islands food webs. 
Figure 3-10 Chlorophyll a map of the Campbell Plateau April 2009, from MODIS.

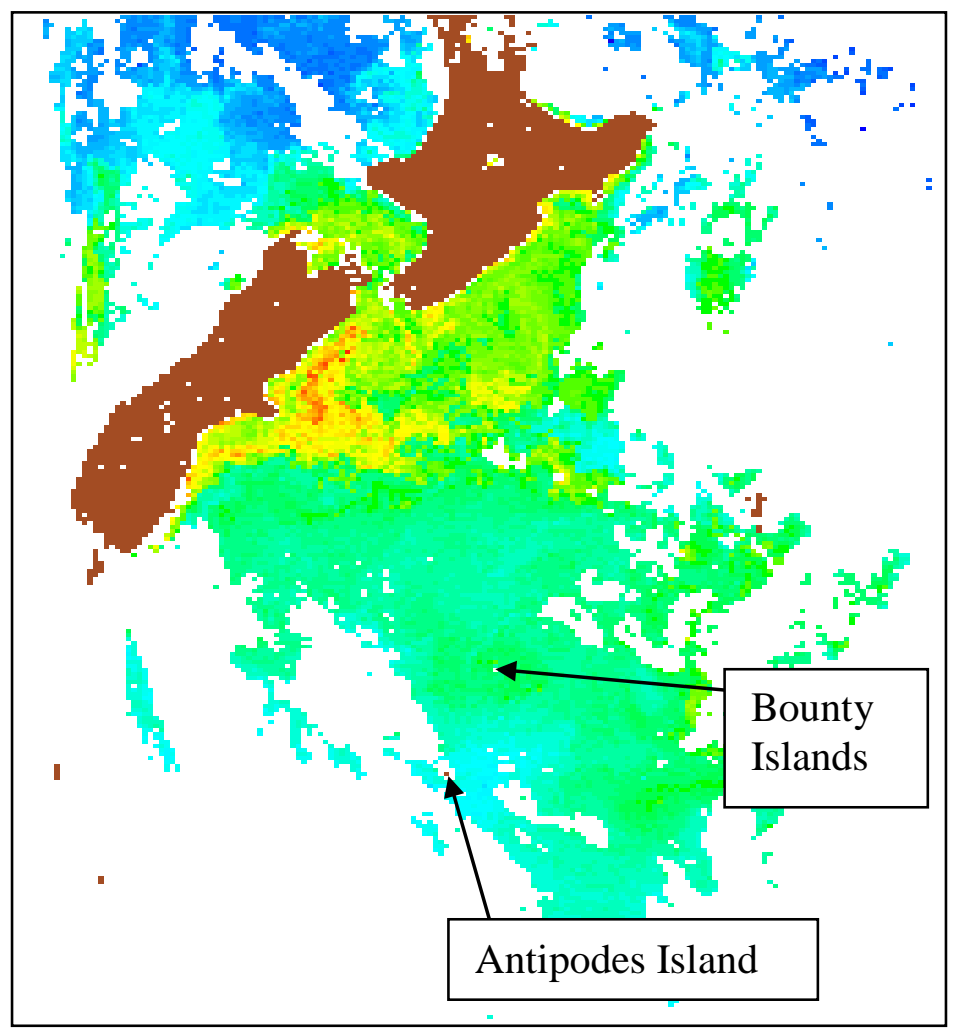


Figure 3-11 Parakeet foraging in penguin guano at Antipodes Island.

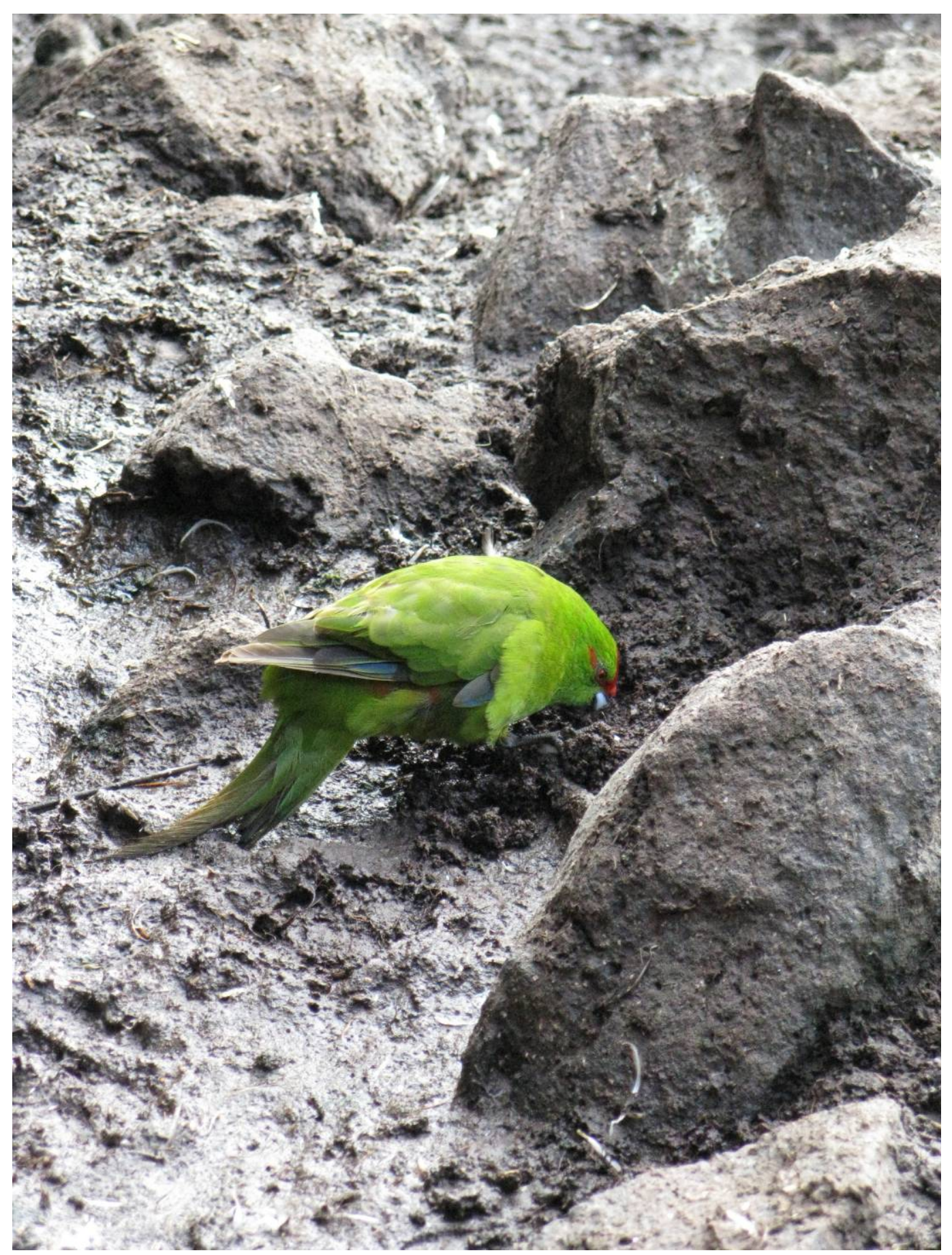


Figure 3-12 Burrow of the spider Pacificana, constructed from fur seal fur on the Bounty Islands.

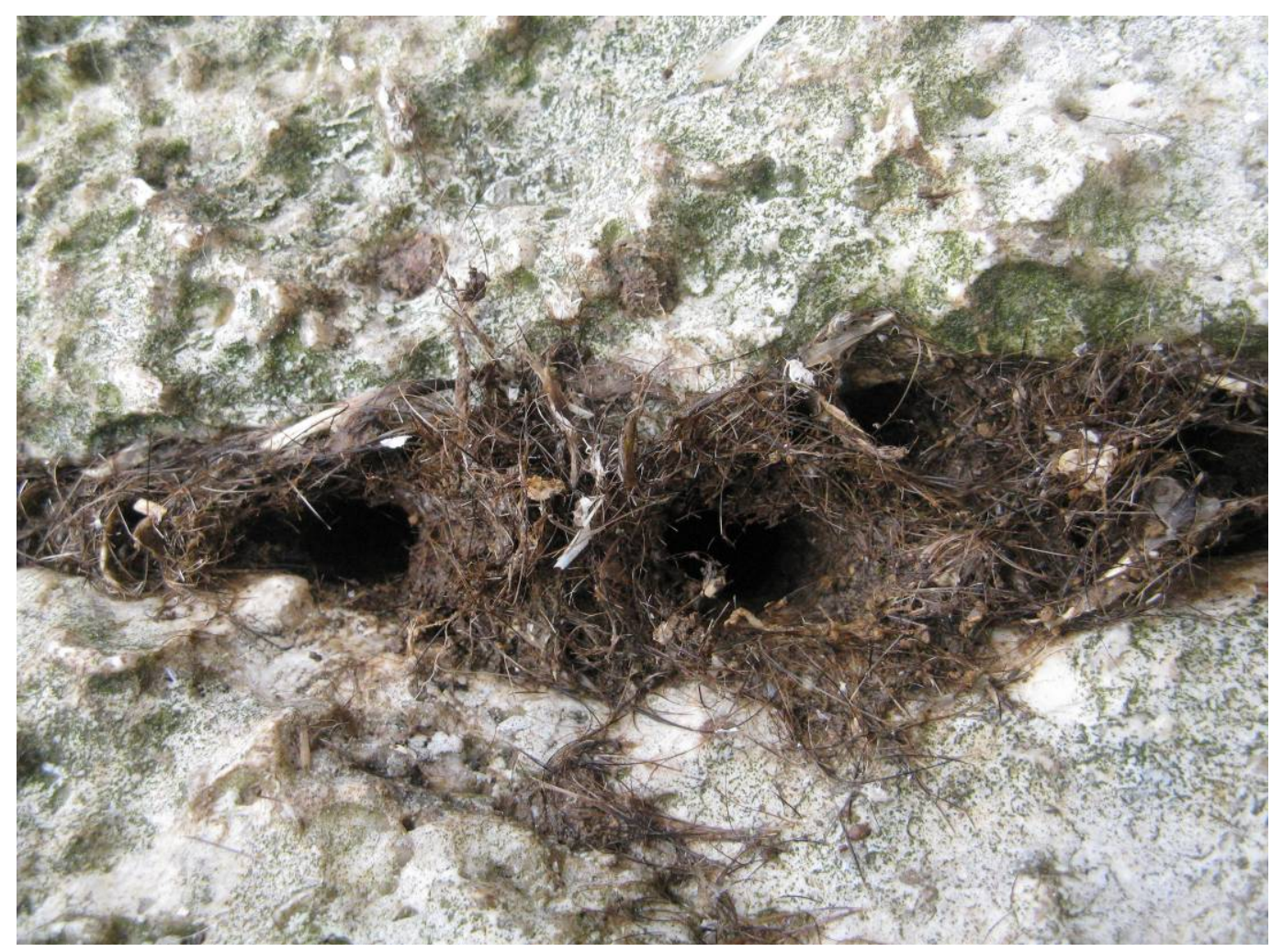




\section{CHAPTER FOUR: General Discussion}

Marine conservation management incorporates a range of planning concepts such as ecosystem-based management, marine protected area planning and marine spatial planning (Halpern et al., 2010). These planning tools have some common principles that seek to ensure the marine environment is sustainably managed and protected in the long-term, and that the spatial characteristics of an ecosystem or habitats are taken into consideration. The basic intent of these management tools is to ensure that the integrity of an ecosystem is intact, the ecosystem is resilient to disturbance and continues to provide for the protection of biodiversity, economic, social and cultural use (Ruckelshaus et al., 2008). Ecosystem based management concepts have become increasingly popular for environmental managers but in practice are challenging to implement (Young et al., 2007, Douvere and Ehler, 2001). This is because ecosystem information is often limited; marine, terrestrial and freshwater ecosystems are usually managed on a sector by sector basis; there is a lack of ecosystem based systematic planning tools; and conflicting views within government agencies on environmental management priorities (Douvere and Ehler, 2001).

The New Zealand Subantarctic Islands are afforded the highest levels of terrestrial protection with all being national nature reserves. Current marine protected areas are limited to the Auckland Islands' territorial sea (note further marine reserves around the Bounty Islands, Antipodes Island and Campbell Island are pending). Despite the intention of the Subantarctic Islands Conservation Management Strategy (Department of Conservation, 1998) to manage the islands from an ecosystem perspective, there has been little progress on implementation, with each realm managed largely in isolation. This may be due in part to the fact that large gaps exist regarding how these ecosystems function and issues relating to over fishing of foraged species and by-catch of seabirds and marine mammals that cross jurisdictional boundaries i.e. outside New Zealand's EEZ. However, it is imperative that ecosystem based information is gathered in order to monitor if the current management regime is effective. 
This thesis has provided the first qualitative and quantitative data on the structure and functioning of the Antipodes and Bounty Islands ecosystems. This important information can help inform future ecosystem based protection and management plans in the region. This research has determined that both islands have strong marine-terrestrial linkages, and that there are high levels of complexity within the food web at Antipodes Island and lower complexity in the Bounty Islands food web. Despite their close proximity (less than one hundred nautical miles apart) these ecosystems are driven by different biological and physical processes, which may have implications for how these islands are impacted and recover from disturbance. It has been clearly demonstrated in my study that the terrestrial and marine environments at these islands require management regimes that are linked to any threats that may alter trophic linkages or the influence of important species and processes. What has been described here may also have some broader implications for the conservation planning and management of the land sea interface and pelagic environments.

\subsection{FOOD WEBS}

Here I propose a food web model for each of the islands based on what was found in this study and known diets of seabirds, marine mammals and fish where data was not available from my study. The Antipodes Island food web (Figure 4-1) can be described as an integrated detritus marine -terrestrial food web where the marine environment and terrestrial environments are linked and driven by three basal carbon sources: macroalgae and phytoplankton in the marine environment and phytoplankton and terrestrial plants in the terrestrial environment. There is evidence for coupling between the pelagic marine and terrestrial environments, where it was found that the marine link to the majority of terrestrial bird species, carrion feeders, parasites and introduced species is not to the nearshore, but to the marine mammals and seabirds that are foraging beyond the islands and that transport nutrients from the pelagic marine environment to the terrestrial environment. 
Subtidal consumers utilised both macroalgae and phytoplankton as energy sources. However, the higher contribution of kelp in consumer diets relative to phytoplankton suggests that rocky reef communities are an important source of carbon at Antipodes Island. Antipodes Island had high carbon isotope values relative to phytoplankton which can also be an indicator that phytoplankton is not the dominant energy source for marine invertebrate consumers (Hobson et al., 1995). Marine invertebrate isotope signatures were closely grouped to macroalgae, which appears to play an important role in the food web. These findings suggest that Antipodes Island is typical of Subantarctic island ecosystems, where carbon from benthic primary producers is an important energy source in the energy budgets of consumers (Dunton, 2001, Kaehler et al., 2000, Kaehler et al., 2006, Jacob et al., 2006).

There is a high level of heterogeneity in the sources of energy where the primary consumers and secondary consumers derive their energy from macroalgae, possibly benthic diatoms and phytoplankton that is transferred through the food chain. The high level of omnivory at Antipodes Island demonstrates that there is no linear pattern in the food web and suggests higher trophic levels are opportunistic and utilise a range of dietary resources dependent on availability.

The level of complexity in the Antipodes Island food web shows that variability of energy resources has been a determining factor in how the food web functions. The heterogeneity suggests that the nearshore marine environment at Antipodes Island is relatively stable, however there may be some seasonal variability. The variation in basal food sources and the high level of omnivory also suggests the Antipodes Island near shore ecosystem functions on the basis of several carbon sources which may allow the ecosystem to be more resilient to seasonal or cyclic fluctuations and disturbance than in systems that are reliant on one source of carbon. It has also been suggested by Rooney et al. (2006) that stable systems are more capable of recovery in shorter timeframes than unstable systems, suggesting that management should be focused on minimising disturbance.

This study did not attempt to describe the whole terrestrial food web and instead focused on the top level predators and primary food sources at the islands. As 
mentioned previously, there is a strong link between the top terrestrial predators and the marine environment. There was a distinct pattern of variability in terrestrial bird diets between marine based sources and terrestrial sources. Terrestrial birds, such as the two species of parakeet on Antipodes Island, foraged on insects in marine mammal haul out sites and areas where bird nesting and moulting occurs. There have been a number of studies showing the importance of allochthonous input from across spatial boundaries (Polis et al., 1997, Hawke and Newman, 2005, Barrett et al., 2005) where mobile consumers such as seabirds and marine mammals have had a influence on terrestrial ecosystem functioning, particularly small islands (Polis and Hurd, 1996, Anderson and Polis, 1998, Sánchez-Piñero and Polis, 2000). This pelagic marineterrestrial coupling suggests that the birds on the islands also show flexibility in their feeding patterns and alternate between marine and terrestrial food sources, a similar pattern to the Snares Islands where Hawke and Newman (2005) found that some terrestrial birds at the island had a proportion of their diet subsidised by marine species. This pattern may also indicate that terrestrial birds have evolved to adapt to seasonal fluctuations in availability of plant based resources by feeding on insects that are present in excrement. In a recent study, Marczak et al. (2007) found that amongst species that utilise marine based subsidies, insectivores benefited the most. By varying their diet, terrestrial birds, such as the parakeets on Antipodes Island, are more likely to be resilient to disturbance than having a solely plant based diet. Interestingly, the introduction of mice to the island has added another dietary supplement to the parakeets' diets, however, mice will be having an impact on the abundance of seed material and insects that are available as a resource (Cuthbert and Hilton, 2004). Although there have been no recent studies on the populations of parakeets at the island, their continued survival despite the introduction of mice suggests that terrestrial birds that have marine subsidies in their diet are able to survive in direct competition with mice so long as their diet is not limited. This provides some evidence that diet variability provides for a more resilient population at the island.

The Bounty Islands displays some similarities but also some significant differences in food web from Antipodes Island (Figure 4-2). The Bounty Islands food web can be categorised as a simple food web with a basal carbon source of 
phytoplankton with little evidence of macroalgae being an important feature. This contrasts to other studies of Subantarctic islands where macroalgae plays an important role in food webs, for example at Prince Edward Islands (Kaehler et al., 2000). It is commonly considered that the nutrient input from high densities of sea birds and marine mammals into the marine environment increases primary production through nutrient enrichment (Wootton, 1991, Lapointe et al., 1992, Harrison, 2006) and macroalgae biomass can also be enhanced (Wootton, 1995, Sánchez-Piñero and Polis, 2000, Kolb et al., 2010). There was evidence to suggest that there is increased phytoplankton production at the Bounty Islands but no evidence to suggest increased macroalgae either through benthic surveys (Freeman et al., 2011) or in the diets of consumers from my study, which may indicate that the Bounty Islands is unique amongst Subantarctic island ecosystems.

Omnivory was also a feature of the Bounty Islands but the food web lacked the complexity of Antipodes Island. The majority of species at the Bounty Islands are solely reliant on a phytoplankton base with the exception of some grazers. A similar situation was found for terrestrial insect species, being fully dependant on a phytoplankton energy base being transferred via seabirds from the pelagic ecosystem.

This study has reached similar conclusions to previous work regarding islands with low terrestrial productivity which are supported by allochthonous input from the marine environment (Polis and Hurd, 1995, Polis and Hurd, 1996, Sánchez-Piñero and Polis, 2000, Bokhorst et al., 2007, Anderson and Polis, 1998). Species are so reliant on the nearshore and pelagic marine environment that the nesting material of the Bounty Island shag is Marginariella parsonii, a subtidal macroalga (Department of Conservation, 2006), and the burrows of the endemic spider Pacificana are constructed of fur seal fur (Figure 3-12). These findings suggest that the Bounty Islands have very strong pelagic-terrestrial and benthic-pelagic interactions. The results of the water sampling suggest that nitrogen and phosphorous concentrations are elevated around the islands due to the large numbers of seabirds and marine mammals that use the islands. The introduction of nutrients from the pelagic ecosystem may be resulting in 
localised increases of phytoplankton production. With little variation in the diets of species at the islands and the possibility that localised phytoplankton production could be a result of nutrients beyond the boundary of the island ecosystem, I suggest that the Bounty Islands ecosystem is very vulnerable to disturbance either via environmental change or the removal of top predators. For example, if there were significant changes in the seabird or marine mammal populations on the Bounty Islands, this would likely have important implications for the species that are directly or indirectly reliant on them, including the Pacificana spider and filter-feeding marine invertebrates. It is possible that when New Zealand fur seals were virtually eradicated from the Bounty Islands following commercial sealing in the 1800s, the marine environment surrounding the islands could have shifted in structure and function considerably, and then perhaps shifted again as the seal population has recovered.

The results presented in this thesis clearly demonstrate that the linkages are very strong between the terrestrial and marine environments at these islands. I therefore propose that they are part of the same ecosystem and are intricately linked via strong feeding relationships.

This study has clearly demonstrated that the Antipodes and Bounty Island ecosystems are strongly influenced by the marine environment and that the flow and flux of environmental conditions, coupled with species loss through the direct and indirect effects of use of the marine environment, may have an impact on the islands' food webs if the top predator populations decrease.

Anthropogenic impacts in the marine environment can come from a variety of sources, such as fishing, mining and sedimentation (MacDiarmid et al., 2012). The results presented here indicate that both the islands' food webs may be negatively impacted by disturbance at either a very local scale or by pressures on species that are occurring in other ecosystems. The implications of the complex interactions between the marine and terrestrial environment at the islands coupled with the possibility that threats on the island ecosystems are not isolated to local stressors indicate very clearly the requirement to manage the islands from a multi ecosystem perspective. These islands and areas that display strong 
linkages in food webs between the marine ecosystems and terrestrial environment cannot be managed in isolation from one another.

\subsection{IMPLICATIONS FOR ENVIRONMENTAL PROTECTION AND MANAGEMENT}

Environmental management is commonly segregated between the marine, terrestrial and freshwater environments without considering the possibility of interactions (Beck, 2003). Whilst the idea of ecosystem based management in the marine environment has been embraced as a concept, little attention has been given to determining how ecosystem based management would work in practice. Whilst there have been some significant advances in the implementation of ecosystem based management within the marine environment in recent years and marine management is increasingly recognising the impacts of terrestrial-based activities (e.g. Morrison et al., 2009), in the terrestrial environment a heavy weighting is usually given towards terrestrial species and habitat management without the consideration of the potential marine connection (Stoms et al., 2005).

There have been significant advances in the size of the conservation estate of up to $30 \%$ of New Zealand's terrestrial environment being protected in National Parks (Craig et al., 2000) and many advances in methods for pest management and eradications (Glen et al., 2013). However, there has been relatively little progress made on marine protection, with approximately $1 \%$ of our exclusive economic zone protected in no-take marine reserves (Ministry for the Environment, 2012) and approximately 30\% designated as benthic protection areas that have restrictions on benthic trawling and dredging but do not include any other protection from benthic disturbance (Leathwick et al., 2008) .

Marine protected areas (MPAs) in New Zealand have been developed in a variety of ways. Historically, the process has been ad hoc via proposals by incorporated groups or community formed groups such as the Guardians of Fiordland Fisheries (Mulcahy et al., 2012) . More recently MPA proposals have been developed under the guidance of the Marine Protected Areas Policy and 
Implementation Plan (Ministry of Fisheries and Department of Conservation, 2008) which focuses on the protection of biodiversity using a range of legislative tools but clearly states that the intent of the policy is not comprehensive marine management and does not include species interactions. In essence, protected areas are established largely in isolation from broader environmental management. The policy utilises community forums to provide recommendations to government based on a set of principles requiring the protection of representative examples of a full range of habitats and ecosystems in a network. The MPA forums are provided with a habitat classification that determines what habitats should be protected using physical surrogates on the assumption that physical habitat will determine the composition of overlying biota and therefore capture the areas biodiversity. Freeman et al. (2011) in their study at Antipodes Island and the Bounty Islands clearly identified the shortcomings of the classification in the Subantarctic region, demonstrating that whilst the islands had the same types of physical habitats they were completely different in community composition.

An evident problem in MPA planning and implementation in New Zealand is that whilst the policy incorporates the concept of ecosystems it is not an ecosystem based policy. The current "network", or what is actually in practice largely isolated small marine reserves (Willis, in press), is not representative of a full range of habitats or ecosystems (Department of Conservation and Ministry of Fisheries, 2011). Representation of actual ecosystems and the processes that sustain them are not evident in the network, either this is due to the information not being available for community forums or decision makers, or the MPAs have been a product of geopolitical decision making and not biodiversity conservation. For example, at Antipodes Island and the Bounty Islands there are currently recommendations being considered for large MPAs surrounding the islands. At Antipodes Island there is a proposed full no-take marine reserve that encompasses the territorial sea (217 $286 \mathrm{ha}$ ) and the Bounty Islands proposal covers 58\% of the territorial sea (104 625 ha) (Subantarctic Marine Protection Planning Forum, 2009) 
This study has clearly demonstrated that whilst these are large marine reserves, they will not protect the integrity of the island ecosystem and maintain all the connections between the land and the sea. For example, species such as Salvin's albatross and New Zealand fur seals that bring significant nutrients to the islands, will forage beyond the boundaries of the proposed marine reserves (Spear et al., 2003, Baylis and Nichols, 2009). Foraging zones and foraged species also need to be maintained at levels that allow for the link between the pelagic marine environment and the terrestrial environment and ensure the cycle of nutrient transfer back to the islands.

In order to undertake ecosystem based MPA planning, further policy and legislative development is required based around objectives beyond the principles of the current policy context, that take into consideration integrated management of the ocean, rivers and terrestrial environment. This does not mean a "lock it up" scenario as is often feared by those that utilise the marine environment for recreation or economic gain. What this means is that MPAs need to be part of a broader concept of marine spatial planning where the use of the marine environment is considered along with management and protection as part of an ecosystem management package. For example the Great Barrier Reef Marine Park Authority (GBRMPA) has implemented a zoning plan containing zones with different objectives and restrictions on specific activities that can be undertaken within the zones (Olsson et al., 2008). In the Belgium North Sea there was impetus for the development of a marine spatial plan following prospective development of offshore energy production, as well from the need to identify and protect conservation areas (Hauraki Gulf Forum, 2011).

Whilst there have been advances in systematic conservation planning in the last decade to begin to take into account heterogeneity and ecosystem processes into conservation planning (Possingham et al., 2005), environmental realms continue to be treated separately (Beger et al., 2010). There clearly needs to be a shift from sectoral-based conservation and management planning to a process of integrated collaborative planning where marine, terrestrial and freshwater sectors work together to provide for more effective ecosystem based management. It is also imperative that international science and government collaboration is part of 
integrated planning and species management, as the species that are important in linking the terrestrial and marine realms often cross jurisdictional boundaries.

One hypothetical scenario could be that environmental agencies in collaboration with sectoral interests define a clear set of conservation and management objectives based on an integrated planning approach that encompass all three environments. Objectives would be required that challenge traditional methods in the way we collect, use and disseminate information. Some aspects to consider in setting objectives could be that the relevant scientific and use information is not based on defining what the boundaries of habitat or ecosystem are, as there are inherent difficulties in placing boundaries around ecosystems due to issues of scale (Post et al., 2007a) or defining individual habitats or species abundance. I would suggest that using information that describes ecological functioning, the processes that drive the ecosystem system and the cumulative impact of pressures on the system provides a solid ecological baseline to progress from. Initiating environmental planning based on information that identifies the nature and extent of ecological interactions, the processes that support the links in the system and then identifying the direct, indirect and cumulative pressures that may destabilise the system would provide a robust biological foundation to build an integrated conservation and management framework around.

The economic context of a planning scenario is also very important to consider as an objective but not one where the focus is on minimizing impacts on existing users as is the current policy context. The valuation of natural capital is increasingly becoming a more important factor in "balancing” the value of extractive use against the value of the ecosystem services that the environment provides (Remoundou et al., 2009). It is therefore critical in any conservation and management planning that the monetary and non-monetary values of the environment are defined and calculated so that any trade offs are undertaken on the basis of a full set of economic information and not primarily based on the analysis of economic loss to users (Ministry of Fisheries and Department of Conservation, 2008). In order to bring this information into a usable form there would need to be an objective to disseminate information in a way that can be used for aiding and optimising planning and for open public use. 
There are a number of decision support tools that have been used for systematic conservation planning such as Zonation (Moilanen et al., 2009), Marxan (Watts et al., 2009) and C-Plan (Pressey et al., 2009), but very few allow for non-experts to use the tools and there is often a gap between the science and government agencies' use of the systematic planning tools (Levin et al., 2013). The reality of conservation planning is that there is an emphasis on minimising the effects of conservation on existing users. Historically this has been undertaken through negotiation based on conservation and management through "horse trading" which often leads to outcomes that are not optimal from either a conservation or an economic perspective. Therefore an objective for any conservation planning scenario must include decision support tools that optimise conservation, economic and social outcomes.

Ideally, the legislative framework would provide for stronger linkages between environmental management legislation but legislation is often sectoral as is the case in New Zealand (Mulcahy et al., 2012). Whilst I am not going to explore the details of the legislative and implementation context here I would recommend further investigation on how ecosystem based objectives could be incorporated into a framework that allows for New Zealand's natural resources to be managed in an integrated manner. This requires significant commitment and change of perspective by government, the commercial sector and communities, to start building the foundations for ensuring that future generations are supported by and are a part of an intact, resilient and sustainable environment.

\subsection{SUMMARY AND CONCLUSIONS}

In summary, this study has provided new information and a detailed insight into the ecosystem functioning at Antipodes Island and the Bounty Islands. I have described the food webs of the islands and the processes that drive the structure of these webs and demonstrated that clear marine terrestrial linkages occur. The research that I have undertaken has highlighted that there are gaps in the current management practices at these islands and further consideration needs to be 
given to transitioning from a habitat and species based management approach to an ecosystem based approach. In light of this research I recommend that further work needs to be undertaken regarding the gaps in current management regimes where environmental and governance realms are managed in isolation from each other. This will allow environmental managers to reduce threats at the ecosystem level to minimise biodiversity loss and the risk of degradation of ecosystems to protect New Zealand's long-term biodiversity, social, cultural and economic prosperity. 
Figure 4-1 Antipodes Island food web showing the connections between seabird and marine mammal foraging sites, the nearshore marine environment and terrestrial environments.

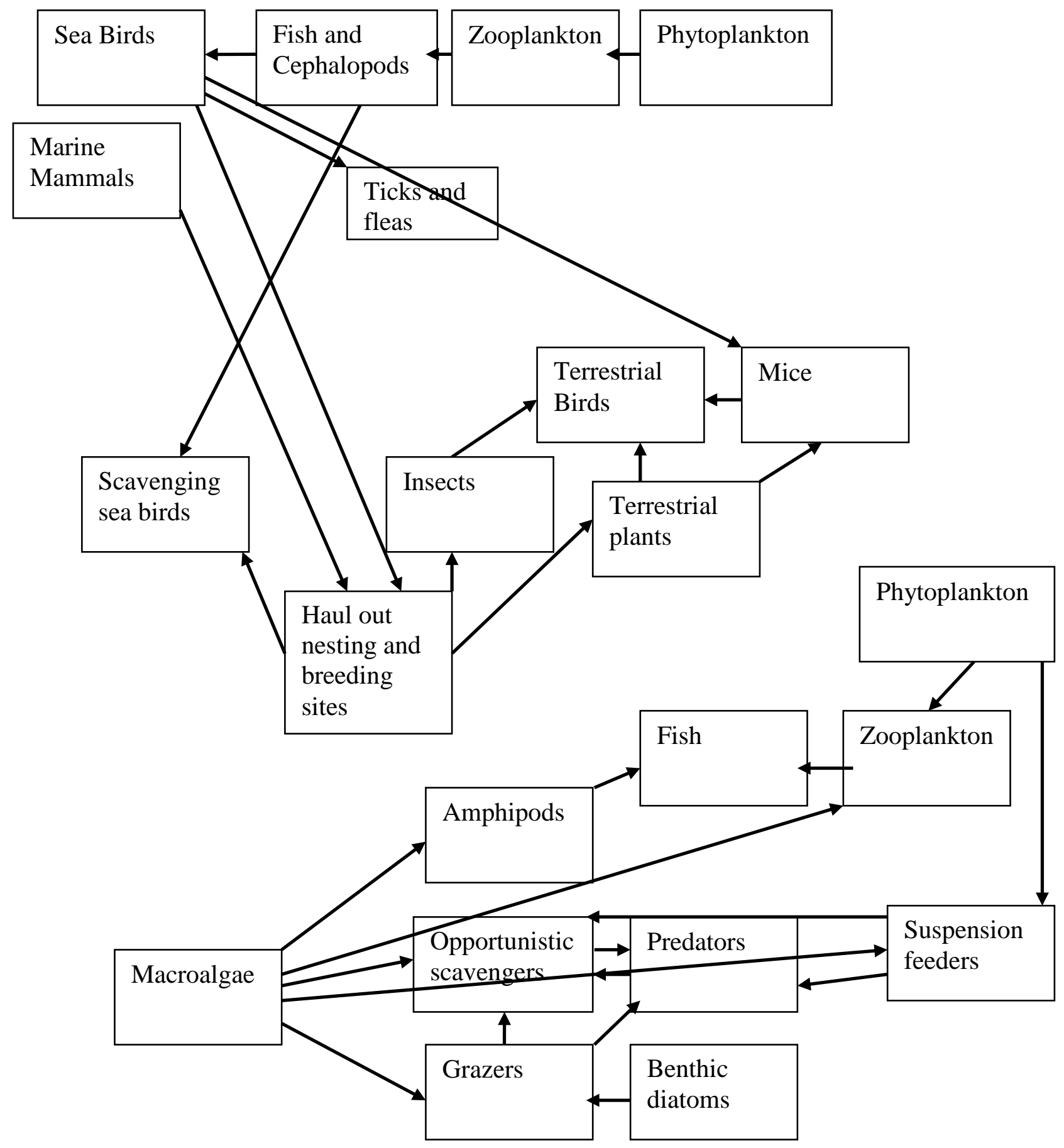


Figure 4-2 Bounty Islands food web showing the connections between seabird and marine mammal foraging sites, the nearshore marine environment and terrestrial environments.

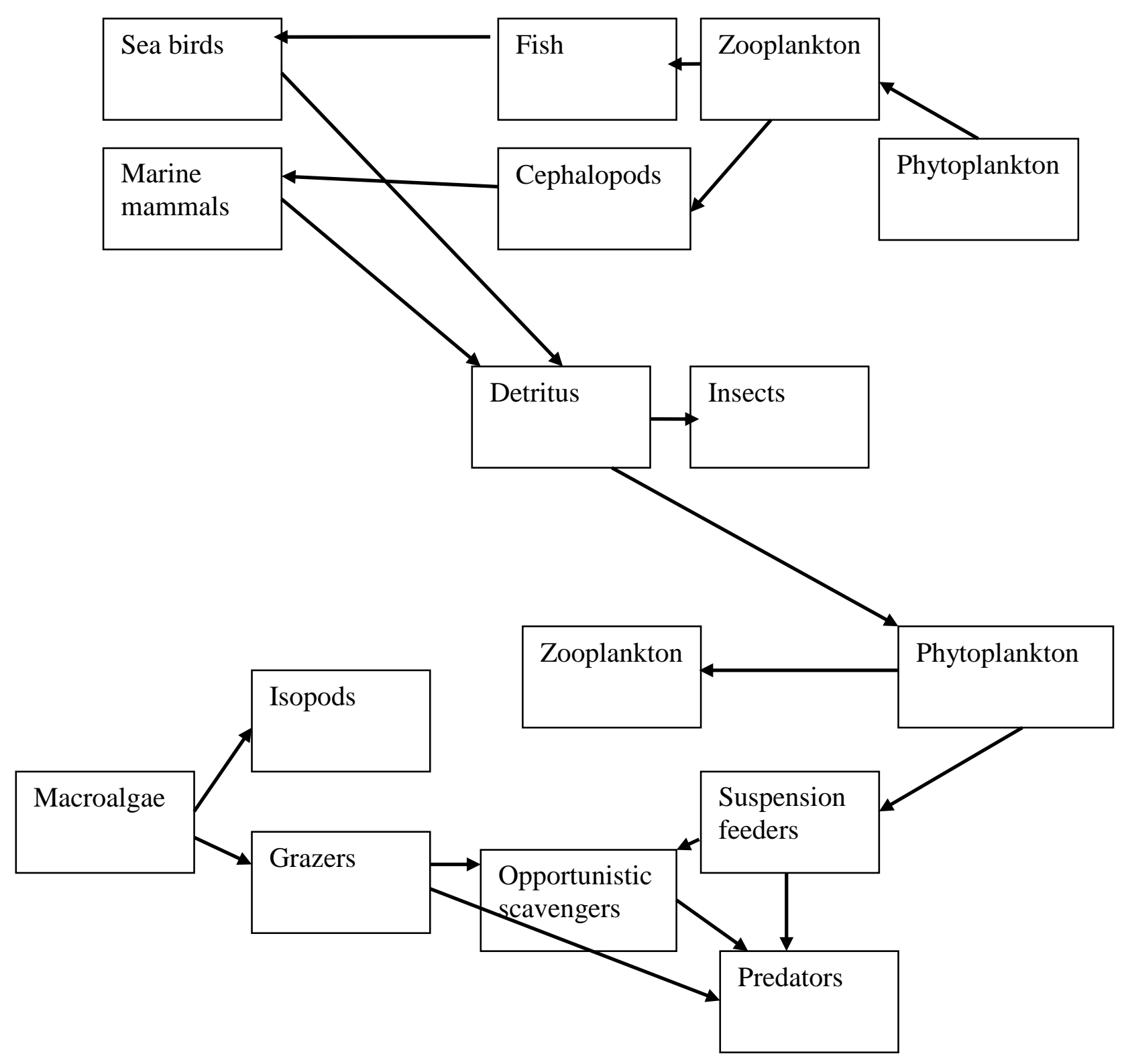




\section{LITERATURE CITED}

ADAMS, C. J. \& CULLEN, D. J. 1978. Potassium-argon ages of granites and metasediments from the Bounty Islands area, northwest Pacific ocean. Journal of the Royal Society of New Zealand, 8, 127-132.

AMEY, J., LORD, J. M. \& DE LANGE, P. 2007. First record of a vascular plant from the Bounty Islands: Lepidium oleraceum (nau, Cook's scurvy grass) (Brassicaceae). New Zealand Journal of Botany, 45, 87-90.

ANDERSON, W. B. \& POLIS, G. A. 1998. Marine subsidies of island communities in the Gulf of California: Evidence from stable carbon and nitrogen isotopes. OIKOS, 81, 75-80.

BABCOCK, R. C., KELLY, S., SHEARS, N. T., WALKER, J. W. \& WILLIS, T. J. 1999. Changes in community structure in temperate marine reserves. Marine Ecology Progress Series, 189, 125-134.

BARRETT, K., ANDERSON, W. B., WAIT, D. A. \& GRISMER, L. L. 2005. Marine subsidies alter the diet and abundance of insular and coastal lizard populations. Oikos, 109, 145-153.

BARRY, J. P. \& DAYTON, P. K. 1991. Physical heterogeneity and the organization of marine communities. Ecological heterogeneity. Springer.

BAYLIS, A. M. M. \& NICHOLS, P. D. 2009. Milk fatty acids predict the foraging locations of the New Zealand fur seal: continental shelf versus oceanic waters. Marine Ecology Progress Series, 380, 271-286.

BECK, M. W. 2003. The sea around: conservation planning in marine regions. Drafting a Conservation Blueprint: A Practitioner's Guide to Planning for Biodiversity, 319-44.

BEGER, M., GRANTHAM, H. S., PRESSEY, R. L., WILSON, K. A., PETERSON, E. L., DORFMAN, D., MUMBY, P. J., LOURIVAL, R., BRUMBAUGH, D. R. \& POSSINGHAM, H. P. 2010. Conservation planning for connectivity across marine, freshwater, and terrestrial realms. Biological Conservation, 143, 565-575.

BLANKLEY, W. \& BRANCH, G. 1985. Ecology of the limpet Nacella delesserti (Philippi) at Marion Island in the sub-Antarctic southern ocean. Journal of Experimental Marine Biology and Ecology, 92, 259-281.

BOKHORST, S., HUISKES, A., CONVEY, P. \& AERTS, R. 2007. External nutrient inputs into terrestrial ecosystems of the Falkland Islands and the Maritime Antarctic Region. Polar Biology, 30, 1315-1321.

BOOTH, J. D. 2004. The marine ecosystem of New Zealand's subantarctic islands and their surrounding plateaus. Wellington, New Zealand: National Institute of Water and Atmospheric Research.

BOSMAN, A. L. \& HOCKEY, P. A. R. 1986. Seabird guano as a determinant of rocky intertidal community structure. Marine Ecology Progress Series, 32, 247-257.

BRADFORD-GRIEVE, J., CHANG, F., GALL, M., PICKMERE, S. \& RICHARDS, F. 1997. Size-fractionated phytoplankton standing stocks and primary production during austral winter and spring 1993 in the Subtropical Convergence region near New Zealand. New Zealand journal of marine and freshwater research, 31, 201-224. 
BRADFORD-GRIEVE, J. M., PROBERT, P. K., NODDER, S. D., THOMPSON, D., HALL, J., HANCHET, S., BOYD, P., ZELDIS, J., BAKER, A. N., BEST, H. A., BROEKHUIZEN, N., CHILDERHOUSE, S., CLARK, M., HADFIELD, M., SAFI, K. \& WILKINSON, I. 2003. Pilot trophic model for subantarctic water over the Southern Plateau, New Zealand: A low biomass, high transfer efficiency system. Journal of Experimental Marine Biology and Ecology, 289, 223-262.

BRETT, M. T. \& GOLDMAN, C. R. 1997. Consumer versus resource control in freshwater pelagic food webs. Science, 275, 384-386.

BROWMAN, H. I., CURY, P. M., HILBORN, R., JENNINGS, S., LOTZE, H. K., MACE, P. M., MURAWSKI, S., PAULY, D., SISSENWINE, M. \& STERGIOU, K. I. 2004. Ecosystem-based Management. Marine Ecology Progress Series, 274, 269-303.

BUSTAMANTE, R. H. \& BRANCH, G. M. 1996. The dependence of intertidal consumers on kelp-derived organic matter on the west coast of South Africa. Journal of Experimental Marine Biology and Ecology, 196, 1-28.

CARABEL, S., GODÍNEZ-DOMÍNGUEZ, E., VERÍSIMO, P., FERNÁNDEZ, L. \& FREIRE, J. 2006. An assessment of sample processing methods for stable isotope analyses of marine food webs. Journal of Experimental Marine Biology and Ecology, 336, 254-261.

CHANG, F. H. \& GALL, M. 1998. Phytoplankton assemblages and photosynthetic pigments during winter and spring in the Subtropical Convergence region near New Zealand. New Zealand journal of marine and freshwater research, 32, 515-530.

CHEREL, Y. 2008. Isotopic niches of emperor and Adelie penguins in Adelie Land, Antarctica. Marine Biology, 154, 813-821.

CHEREL, Y. \& HOBSON, K. A. 2007. Geographical variation in carbon stable isotope signatures of marine predators: a tool to investigate their foraging areas in the Southern Ocean. Marine Ecology Progress Series, 329, 281287.

CHEREL, Y., JAEGER, A., ALDERMAN, R., JAQUEMET, S., RICHARD, P., WANLESS, R. M., PHILLIPS, R. A. \& THOMPSON, D. R. 2012. A comprehensive isotopic investigation of habitat preferences in nonbreeding albatrosses from the Southern Ocean. Ecography.

CHISWELL, S. M., BRADFORD-GRIEVE, J., HADFIELD, M. G. \& KENNAN, S. C. 2013. Climatology of surface chlorophyll a, autumn, winter and spring blooms in the southwest Pacific Ocean. Journal of Geophysical Research: Oceans.

CHOWN, S., GREMMEN, N. \& GASTON, K. 1998. Ecological biogeography of southern ocean islands: species-area relationships, human impacts, and conservation. The American Naturalist, 152, 562-575.

CHOWN, S. L., RODRIGUES, A. S. L., GREMMEN, N. J. M. \& GASTON, K. J. 2001. World heritage status and conservation of southern ocean islands. Conservation Biology, 15, 550-557.

CHRISTENSEN, J. T. \& RICHARDSON, K. 2008. Stable isotope evidence of long-term changes in the North Sea food web structure. Marine Ecology Progress Series, 368, 1-8.

CLARK, M. R. 1985. The food and feeding of seven fish species from the Campbell Plateau, New Zealand. New Zealand Journal of Marine and Freshwater Research, 19, 339-363. 
CLARK, W. C. 1971. Pycnogonida of the Antipodes Islands. New Zealand Journal of Marine and Freshwater Research, 5, 427-452.

CRAIG, J., ANDERSON, S., CLOUT, M., CREESE, B., MITCHELL, N., OGDEN, J., ROBERTS, M. \& USSHER, G. 2000. Conservation issues in New Zealand. Annual Review of Ecology and Systematics, 61-78.

CREESE, R. G. 1988. Ecology of molluscan grazers and their interactions with marine algae in northeastern New Zealand: A review. New Zealand Journal of Marine and Freshwater Research, 22, 427-444.

CROWDER, L. B., REAGAN, D. P. \& FRECKMAN, D. W. 1996. Food web dynamics and applied problems. Food Webs. Springer.

CUTHBERT, R. \& HILTON, G. 2004. Introduced house mice Mus musculus: a significant predator of threatened and endemic birds on Gough Island, South Atlantic Ocean? Biological Conservation, 117, 483-489.

DARIMONT, C. T., PAQUET, P. C. \& REIMCHEN, T. E. 2008. Landscape heterogeneity and marine subsidy generate extensive intrapopulation niche diversity in a large terrestrial vertebrate. Journal of Animal Ecology, 78, 126-133.

DENNO, R. F., MITTER, M. S., LANGELLOTTO, G. A., GRATTON, C. \& FINKE, D. L. 2004. Interactions between a hunting spider and a webbuilder: consequences of intraguild predation and cannibalism for prey suppression. Ecological Entomology, 29, 566-577.

DEPARTMENT OF CONSERVATION 1998. Conservation management strategy: Subantarctic Islands 1998-2008. Invercargill, New Zealand.

DEPARTMENT OF CONSERVATION AND MINISTRY OF FISHERIES. 2011. Coastal marine habitats and marine protected areas in the New Zealand Territorial Sea: a broad scale gap analysis. Wellington Department of Conservation.

DEPARTMENT OF CONSERVATION 2006. Marine protection for the New Zealand Subantarctic Islands: A background resource document. Wellington, New Zealand: Department of Conservation.

DOUVERE, F. \& EHLER, C. 2001. Ecosystem-based marine spatial management: An evolving paradigm for the management of coastal and marine places. Management, 44, 563-566.

DREVER, M. C., BLIGHT, L. K., HOBSON, K. A. \& BERTRAM, D. F. 2000. Predation on seabird eggs by Keen's mice (Peromyscus keeni): using stable isotopes to decipher the diet of a terrestrial omnivore on a remote offshore island. Canadian Journal of Zoology, 78, 2010-2018.

DUNTON, K. H. 2001. 15N and 13C measurements of Antarctic Peninsula fauna: Trophic relationships and assimilation of benthic seaweeds. American Zoology, 41, 99-112.

DUNTON, K. H. \& SCHELL, D. M. 1987. Dependence of consumers on macroalgal (Laminaria solidungula) carbon in an arctic kelp community: Delta13C evidence. Marine Biology, 93, 615-625.

ECKMAN, J. E., DUGGINS, D. O. \& SEWELL, A. T. 1989. Ecology of understorey kelp environments. I. Effects of kelps on flow and particle transport near the bottom. Journal of Experimental Marine Biology and Ecology, 129, 173-187.

ELLIS, J. C., FARINA, J. M. \& WITMAN, J. D. 2006. Nutrient transfer from sea to land: the case of gulls and cormorants in the Gulf of Maine. Journal of Animal Ecology, 75, 565-574. 
ELSER, J. J. \& URABE, J. 1999. The stoichiometry of consumer-driven nutrient recycling: theory, observations, and consequences. Ecology, 80, 735-751.

EMERSON, B. 2002. Evolution on oceanic islands: molecular phylogenetic approaches to understanding pattern and process. Molecular ecology, 11, 951-966.

ERSKINE, P. D., BERGSTROM, D. M., SCHMIDT, S., STEWART, G. R., TWEEDIE, C. E. \& SHAW, J. D. 1998. Subantarctic Macquarie Island a model ecosystem for studying animal-derived nitrogen sources using 15N natural abundance. Oecologia, 117, 187-193.

FARIÑA, J., SALAZAR, S., WALLEM, K., WITMAN, J. \& ELLIS, J. 2003. Nutrient exchanges between marine and terrestrial ecosystems: the case of the Galapagos sea lion Zalophus wollebaecki. Journal of Animal Ecology, 72, 873-887.

FOREST, J. \& MCLAY, C. L. 2001. The biogeography and bathymetric distribution of New Zealand hermit crabs (Crustacea: Anomura: Paguridae). Journal of the Royal Society of New Zealand, 31, 687-720.

FRANCOIS, R., ALTABET, M. A., GOERICKE, R., MCCORKLE, D. C., BRUNET, C. \& POISSON, A. 1993. Changes in the $\delta 13 \mathrm{C}$ of surface water particulate organic matter across the subtropical convergence in the SW Indian Ocean. Global Biogeochemical Cycles, 7, 627-644.

FREDRIKSEN, S. 2003. Food web studies in a Norwegian kelp forest based on stable isotope analysis. Marine Ecology Progress Series, 260, 71-81.

FREEMAN, D., COOPER, S., FUNNELL, G. \& NEALE, D. 2011. Nearshore benthic community structure at the Bounty and Antipodes Islands, Subantarctic New Zealand. Polar biology, 34, 1485-1499.

FRY, B. 2006. Stable isotope ecology, Springer.

FRY, B., LUTES, R., NORTHAM, M. \& PARKER, P. L. 1982. A 13C / 12C comparison of food webs in Caribbean seagrass meadows and coral reefs. Aquatic Botany, 14, 389-398.

FRY, B. \& SHERR, E. B. 1984. 13C measurements as indicators of carbon flow in marine and freshwater ecosystems. Contributions in Marine Science, 27, 13-47.

GAGNÉ, J., MANN, K. \& CHAPMAN, A. 1982. Seasonal patterns of growth and storage in Laminaria longicruris in relation to differing patterns of availability of nitrogen in the water. Marine Biology, 69, 91-101.

GANNES, L. Z., DEL RIO, C. M. \& KOCH, P. 1998. Natural abundance variations in stable isotopes and their potential uses in animal physiological ecology. Comparative Biochemistry and Physiology, 119A, 725-737.

GILI, J.-M. \& COMA, R. 1998. Benthic suspension feeders: their paramount role in littoral marine food webs. Trends in Ecology \& Evolution, 13, 316321.

GLEN, A. S., ATKINSON, R., CAMPBELL, K. J., HAGEN, E., HOLMES, N. D., KEITT, B. S., PARKES, J. P., SAUNDERS, A., SAWYER, J. \& TORRES, H. 2013. Eradicating multiple invasive species on inhabited islands: the next big step in island restoration? Biological Invasions, 1-15.

GOLDSTIEN, S. J., GEMMELL, N. J. \& SCHIEL, D. R. 2009. Colonisation and connectivity by intertidal limpets among New Zealand, Chatham and SubAntarctic Islands. I. Genetic connections. Marine Ecology Progress Series, 388, 111-119. 
GOLOVKIN, A. 1967. The effect of colonial seabirds on the development of phytoplankton. Okeanologya, 7, 672-682.

GREENE, T. C. 1995. Aspects of the ecology of Antipodes Island Parakeet (Cyanoramphus unicolor) and Reischek's Parakeet (C. novaezelandiae hochstetten) on Antipodes Island, October-November 1995.

HAIRSTON, J., NELSON G \& HAIRSTON, S., NELSON G 1997. Does food web complexity eliminate trophic-level dynamics? The American Naturalist, 149, 1001-1007.

HALPERN, B. S., LESTER, S. E. \& K.L., M. 2010. Placing marine protected areas onto the ecosystem-based management seascape. Proceedings of the National Academy of Sciences.

HARDING, J. S., HAWKE, D. J., HOLDAWAY, R. N. \& WINTERBOURN, M. J. 2004. Incorporation of marine-derived nutrients from petrel breeding colonies into stream food webs. Freshwater Biology, 49, 576586.

HARRISON, S. A. 2006. The influence of seabird-derived nutrients on island ecosystems in the oligotrophic marine waters of south-western Australia. MSc, Edith Cowan University.

HAURAKI GULF FORUM 2011. Spatial Planning for the Gulf: An interntional review if marine spatial planning initiatives and application to the Hauraki Gulf. Auckland, New Zealand.

HAWKE, D. J. \& HOLDAWAY, R. N. 2009. Nutrient sources for forest birds captured within an undisturbed petrel colony, and management implications. Emu, 109, 163-169.

HAWKE, D. J. \& MISKELLY, C. M. 2009. 13C and 15N enrichment in snipe (Coenocorypha spp.) from islands across the New Zealand biogeographic region. Journal of the Royal Society of New Zealand, 39, 35-42.

HAWKE, D. J. \& NEWMAN, J. 2005. Using isotopic analysis to identify incorporation of marine nutrients in terrestrial birds at Snares Islands. Notornis, 52, 108-111.

HAY, C. H., ADAMS, N. M. \& PARSONS, M. J. 1985. Marine algae of the subantarctic islands of New Zealand. National Museum of New Zealand Miscellaneous Series number, 11.

HAYWARD, B. W. \& MORLEY, M. S. 2005. Zonation and biogeography of the intertidal biota of subantarctic Campbell and Auckland Islands, New Zealand. Records of the Auckland Museum, 42, 7-33.

HEATH, R. \& BRADFORD, J. 1980. Factors affecting phytoplankton production over the Campbell Plateau, New Zealand. Journal of plankton research, 2, 169-181.

HEYMANS, J. J. \& BAIRD, D. 2000. Network analysis of the northern Benguela ecosystem by means of Netwrk and EcoPath. Ecological Modelling, 131, 97-119.

HIGGINS, P. J. 1996. Handbook of Australian, New Zealand \& Antarctic birds. Vol. 3, Snipe to pigeons.

HOBSON, K., AMBROSE, W. \& RENAUD, P. 1995. Sources of primary production, benthic-pelagic coupling, and trophic relationships within the Northeast Water Polynya: insights from D13C and D15N analysis. Marine ecology progress series. Oldendorf, 128, 1-10. 
HOBSON, K. A. 1993. Trophic relationships among high Arctic seabirds: insights from tissue-dependent stable-isotope models. MARINE ECOLOGY-PROGRESS SERIES, 95, 7-7.

HOBSON, K. A., GLOUTNEY, M. L. \& GIBBS, H. L. 1997a. Preservation of blood and tissue samples for stable-carbon and stable-nitrogen isotope analysis. Canadian Journal of Zoology, 75, 1720-1723.

HOBSON, K. A., SEASE, J. L., MERRICK, R. L. \& PIATT, J. F. 1997b. Investigating trophic relationships of pinnipeds in Alaska and Washington using stable isotope ratios of nitrogen and carbon. Marine Mammal Science, 13, 114-132.

HOBSON, K. A. \& WELCH, H. E. 1992. Determination of trophic relationships within a high Arctic marine food web using delta13C and delta15N analysis. Marine Ecology Progress Series, 84, 9-18.

HOEKSTRA, T. W. 1992. Toward a unified ecology, Columbia University Press.

HOLLING, C. S. 1973. Resilience and stability of ecological systems. Annual review of ecology and systematics, 4, 1-23.

HORNE, R. 1985. Diet of royal and rockhopper penguins at Macquarie Island. Етu, 85, 150-156.

HORNING, D. S. 1986. 1978 expedition to the Bounty and Antipodes Islands, New Zealand. Wellington, New Zealand: A report submitted to the Wildlife Service, Department of Internal Affairs.

HOWARTH, R. W. 1988. Nutrient limitation of net primary production in marine ecosystems. Annual Review of Ecology and Systematics, 89-110.

JABIOL, J., MCKIE, B. G., BRUDER, A., BERNADET, C., GESSNER, M. O. \& CHAUVET, E. 2013. Trophic complexity enhances ecosystem functioning in an aquatic detritus-based model system. Journal of Animal Ecology.

JACK, L. \& WING, S. R. 2011. Individual variability in trophic position and diet of a marine omnivore is linked to kelp bed habitat. Marine Ecology Progress Series, 443, 129-139.

JACOB, U., BREY, T., FETZER, I., KAEHLER, S., MINTENBECK, K., DUNTON, K., BEYER, K., STRUCK, U., PAKHOMOV, E. A. \& ARNTZ, W. E. 2006. Towards the trophic structure of the Bouvet Island marine ecosystem.

JACOB, U., MINTENBECK, K., BREY, T., KNUST, R. \& BEYER, K. 2005. Stable isotope food web studies: A case for standardized sample treatment. Marine Ecology Progress Series, 287, 251-253.

KAEHLER, S., PAKHOMOV, E. A., KALIN, R. M. \& DAVIS, S. 2006. Trophic importance of kelp-derived suspended particulate matter in a through-flow sub-Antarctic system. Marine Ecology Progress Series, 316, 17-22.

KAEHLER, S., PAKHOMOV, E. A. \& MCQUAID, C. D. 2000. Trophic structure of the marine food web at the Prince Edward Islands (Southern Ocean) determined by $13 \mathrm{C}$ and $15 \mathrm{~N}$ analysis. Marine Ecology Progress Series, 208, 13-20.

KINGSFORD, M. J., SCHIEL, D. R. \& BATTERSHILL, C. N. 1989. Distribution and abundance of fish in a rocky reef environment at the subantarctic Auckland Islands, New Zealand. Polar Biology, 9, 179-186.

KNOX, G. 1987. The littoral ecology of the subantarctic region: with special reference to the New Zealand subantarctic islands. CNFRA57, 47, 79. 
KOLB, G. S., EKHOLM, J. \& HAMBÄCK, P. A. 2010. Effects of seabird nesting colonies on algae and aquatic invertebrates in coastal waters. Marine Ecology Progress Series, 417, 287-300.

KURLE, C. M. \& WORTHY, G. A. J. 2002. Stable nitrogen and carbon isotope ratios in multiple tissues of the northern fur seal Callorhinus ursinus: Implications for dietary and migratory reconstructions. Marine Ecology Progress Series, 236, 289-300.

KWAK, T. J. \& ZEDLER, J. B. 1997. Food web analysis of southern California coastal wetlands using multiple stable isotopes. Oecologia, 110, 262-277.

LAPOINTE, B. E., LITTLER, M. M. \& LITTLER, D. S. Modification of benthic community structure by natural eutrophication: the Belize Barrier Reef. Seventh International Coral Reef Symposium, 1992 Guam. 323-334.

LAWS, R. M. 1994. History and present status of southern elephant seal populations. Elephant seals: population ecology, behavior, and physiology. University of California Press, Berkeley, 49-65.

LEATHWICK, J., MOILANEN, A., FRANCIS, M., ELITH, J., TAYLOR, P., JULIAN, K., HASTIE, T. \& DUFFY, C. 2008. Novel methods for the design and evaluation of marine protected areas in offshore waters. Conservation Letters, 1, 91-102.

LESAGE, V., MORIN, Y., RIOUX, E., POMERLEAU, C., FERGUSON, S. \& PELLETIER, E. 2010. Stable isotopes and trace elements as indicators of diet and habitat use in cetaceans: predicting errors related to preservation, lipid extraction, and lipid normalisation. Marine Ecology Progress Series, 419, 249-265.

LEVIN, N., WATSON, J. E., JOSEPH, L. N., GRANTHAM, H. S., HADAR, L., APEL, N., PEREVOLOTSKY, A., DEMALACH, N., POSSINGHAM, H. P. \& KARK, S. 2013. A framework for systematic conservation planning and management of Mediterranean landscapes. Biological Conservation, 158, 371-383.

LEVITUS, S., CONKRIGHT, M. E., REID, J. L., NAJJAR, R. G. \& MANTYLA, A. 1993. Distribution of nitrate, phosphate and silicate in the world oceans. Progress in Oceanography, 31, 245-273.

LIDGARD, S. 2008. Predation on marine bryozoan colonies: taxa, traits and trophic groups. MARINE ECOLOGY-PROGRESS SERIES-, 359, 117.

LIKENS, G. 1992. The ecosystem approach: its use and abuse.

LINDEMAN, R. L. 1942. The trophic-dynamic aspect of ecology. Ecology, 23, 399-417.

LINDERBOOM, H. J. 1984. The nitrogen pathway in a penguin rookery. Ecology, 65, 269-277.

LINK, J. 2002. Does food web theory work for marine ecosystems? Marine Ecology Progress Series, 230, 9.

LODER, T. C., GANNING, B. R. \& LOVE, J. A. 1996. Ammonia nitrogen dynamics in coastal rockpools affected by gull guano. Journal of experimental marine biology and ecology, 196, 113-129.

LUNDQUIST, C. J. \& PINKERTON, M. H. 2008. Collation of data for ecosystem modelling of Te Tapuwae o Rongokako Marine Reserve. Wellington, New Zealand: Department of Conservation.

MACDIARMID, A., MCKENZIE, A., STURMAN, J., BEAUMONT, J., MIKALOFF-FLETCHER, S. \& DUNNE, J. 2012. Assessment of 
anthropogenic threats to New Zealand marine habitats. Wellington, New Zealand: NIWA.

MARCZAK, L. B., THOMPSON, R. M. \& RICHARDSON, J. S. 2007. Metaanalysis: trophic level, habitat, and productivity shape the food web effects of resource subsidies. Ecology, 88, 140-148.

MARTINEZ, N. D. \& LAWTON, J. H. 1995. Scale and food-web structure: from local to global. Oikos, 148-154.

MARTINS, G. M., THOMPSON, R. C., HAWKINS, S. J., NETO, A. I. \& JENKINS, S. R. 2008. Rocky intertidal community structure in oceanic islands: scales of spatial variability. Marine Ecology Progress Series, 356, 15-24.

MAY, R. M. 2001. Stability and complexity in model ecosystems, Princeton University Press.

MCCANN, K. S. 2000. The diversity-stability debate. Nature, 405, 228-233.

MCCAULEY, D. J., DESALLES, P. A., YOUNG, H. S., DUNBAR, R. B., DIRZO, R., MILLS, M. M. \& MICHELI, F. 2012. From wing to wing: the persistence of long ecological interaction chains in less-disturbed ecosystems. Scientific reports, 2.

MCCLANAHAN, T. R. \& SALA, E. 1997. A Mediterranean rocky-bottom ecosystem fisheries model. Ecological Modelling, 104, 145-164.

MCCLINTOCK, J. B. 1994. Trophic biology of Antarctic shallow-water echinoderms. Marine ecology progress series. Oldendorf, 111, 191-202.

MCCUTCHAN, J. H., LEWIS, W. M., KENDALL, C. \& MCGRATH, C. 2003. Variation in trophic shift for stable isotope ratios of carbon, nitrogen and sulfur. OIKOS, 102, 378-390.

MCMEANS, B. C., ROONEY, N., ARTS, M. T. \& FISK, A. T. 2013. Food web structure of a coastal Arctic marine ecosystem and implications for stability. Mar Ecol Prog Ser, 482, 17-28.

MICHENER, R. \& LAJTHA, K. 2008. Stable isotopes in ecology and environmental science, Wiley. com.

MICHENER, R. H. \& LAJTHA, K. 2007. Stable isotopes in ecology and environmental science, Blackwell Pub.

MILLER, J. M. J., MILLAR, J. M. J. \& LONGSTAFFE, F. L. F. 2008. Carbonand nitrogen-isotope tissue-diet discrimination and turnover rates in deer mice, Peromyscus maniculatus. Canadian Journal of Zoology, 86, 685691.

MINAGAWA, M. \& WADA, E. 1984. Stepwise enrichment of D15N along food chains: Further evidence and the relation between D15 N and animal age. Geochimica et Cosmochimica Acta, 48, 1135-1140.

MINISTRY FOR THE ENVIRONMENT 2012. Ministry for the Environment Marine protected areas. Wellington New Zealand.

MINISTRY OF FISHERIES \& DEPARTMENT OF CONSERVATION 2008. Marine protected areas: Classification, protection standard and implementation guidelines. Wellington, New Zealand: Ministry of Fisheries and Department of Conservation.

MIZUTANI, H. \& WADA, E. 1988. Nitrogen and carbon isotope ratios in seabird rookeries and their ecological implications. Ecology, 69, 340-349.

MOILANEN, A., KUJALA, H. \& LEATHWICK, J. 2009. The Zonation framework and software for conservation prioritization. Spatial Conservation Prioritization, 196-210. 
MOORE, P. J. 1997. Diet of the yellow-eyed penguin Megadyptes antipodes, South Island, New Zealand, 1991-1993. Marine Ornithology, 25, 17-29.

MORRIS, M., STANTON, B. \& NEIL, H. 2001. Subantarctic oceanography around New Zealand: Preliminary results from an ongoing survey. New Zealand Journal of Marine and Freshwater Research, 35, 499-519.

MORRISON, M. A., LOWE, M. L., PARSONS, D. M., USMAR, N. R. \& MCLEOD, I. M. 2009. A review of land-based effects on coastal fisheries and supporting biodiversity in New Zealand. Wellington, New Zealand: Ministry of Fisheries.

MULCAHY, K., PEART, R., BULL, A. \& SOCIETY, E. D. 2012. Safeguarding Our Oceans: Strengthening Marine Protection in New Zealand, Environmental Defence Society Incorporated.

MUMBY, P. J., STENECK, R. S., EDWARDS, A. J., FERRARI, R., COLEMAN, R., HARBORNE, A. R. \& GIBSON, J. P. 2012. Fishing down a Caribbean food web relaxes trophic cascades. Marine Ecology Progress Series, 445, 13-24.

MURPHY, R. J., PINKERTON, M. H., RICHARDSON, K. M., BRADFORDGRIEVE, J. M. \& BOYD, P. W. 2001. Phytoplankton distributions around New Zealand derived from SeaWiFS remotely-sensed ocean colour data. New Zealand Journal of Marine and Freshwater Research, 35, 343-362.

MURRAY, M. \& VESTJENS, W. 1967. Studies on the ectoparasites of seals and penguins. III. The distribution of the tick Ixodes uriae White and the flea Parapsyllus magellanicus heardi de Meillon on Macquarie Island. Australian Journal of Zoology, 15, 715-725.

NELSON, W. 1994. Distribution of macroalgae in New Zealand-an archipelago in space and time. Botanica Marina, 37, 221-234.

NORKKO, A., THRUSH, S. F., CUMMINGS, V. J., GIBBS, M. M., ANDREW, N. L., NORKKO, J. \& SCHWARZ, A.-M. 2007. Trophic structure of coastal Antarctic food webs associated with changes in sea ice and food supply. Ecology, 88, 2810-2820.

NYSSEN, F., BREY, T., LEPOINT, G., BOUQUEGNEAU, J., DE BROYER, C. \& DAUBY, P. 2002. A stable isotope approach to the eastern Weddell Sea trophic web: Focus on benthic amphipods. Polar Biology, 25, 280287.

OLSSON, P., FOLKE, C. \& HUGHES, T. P. 2008. Navigating the transition to ecosystem-based management of the Great Barrier Reef, Australia. Proceedings of the National Academy of Sciences, 105, 9489-9494.

OWENS, N. 1988. Natural Variations in" N. Advances in marine biology, 24, 389.

PAINE, R. T. 1988. Road Maps of Interactions or Grist for Theoretical Development? Ecology, 69, 1648-1654.

PARK, J., DAY, T. A., STRAUSS, S. \& RUHLAND, C. T. 2007.

Biogeochemical pools and fluxes of carbon and nitrogen in a maritime tundra near penguin colonies along the Antarctic Peninsula. Polar Biology, 30, 199-207.

PAULY, D., CHRISTENSEN, V. \& WALTERS, C. 2000. Ecopath, Ecosim and Ecospace as tools for evaluating ecosystem impact of fisheries. ICES Journal of Marine Science, 57. 
PEAT, N. 2003. Subantarctic New Zealand: a rare heritage, Invercargill, New Zealand, Department of Conservation.

PETERSON, B. J. \& FRY, B. 1987. Stable isotopes in ecosystem studies. Annual Review of Ecology and Systematics, 18, 293-320.

PETERSON, B. J., HOWARTH, R. W. \& GARRITT, R. H. 1985. Multiple stable isotopes used to trace the flow of organic matter in estuarine food webs. Science, 227, 1361-1363.

PHILLIPS, D. L. 2001. Mixing models in analyses of diet using multiple stable isotopes: A critique. Oecologia, 127, 166-170.

PHILLIPS, D. L. 2012. Converting isotope values to diet composition: the use of mixing models. Journal of Mammalogy, 93, 342-352.

PHILLIPS, D. L. \& GREGG, J. W. 2001. Uncertainty in source partitioning using stable isotopes. Oecologia, 127, 171-179.

PHILLIPS, D. L. \& GREGG, J. W. 2003. Source partitioning using stable isotopes: coping with too many sources. Oecologia, 136, 261-269.

PHILLIPS, J. \& HURD, C. 2003. Nitrogen ecophysiology of intertidal seaweeds from New Zealand: N uptake, storage and utilisation in relation to shore position and season. Marine ecology. Progress series, 264, 31-48.

PICKETT, S. T. \& CADENASSO, M. 2002. The ecosystem as a multidimensional concept: meaning, model, and metaphor. Ecosystems, 5, $1-10$.

PICKETT, S. T., KOLASA, J. \& JONES, C. G. 2010. Ecological understanding: the nature of theory and the theory of nature, Academic Press.

PIMM, S. \& LAWTON, J. 1978. On feeding on more than one trophic level. Nature, 275, 542-544.

PINKERTON, M. H., LUNDQUIST, C. J., DUFFY, C. A. J. \& FREEMAN, D. J. 2008. Trophic modelling of a New Zealand rocky reef ecosystem using simultaneous adjustment of diet, biomass and energetic parameters. Journal of Experimental Marine Biology and Ecology, 367, 61-266.

POLIS, G. A., ANDERSON, W. B. \& HOLT, R. D. 1997. Toward an integration of landscape and food web ecology: the dynamics of spatially subsidized food webs. Annual Review of Ecology and Systematics, 28, 289-316.

POLIS, G. A., HOLT, R. D., MENGE, B. A. \& WINEMILLER, K. O. 1996. Time, space, and life history: influences on food webs. Food Webs. Springer.

POLIS, G. A. \& HURD, S. D. 1995. Extraordinarily high spider densities on islands: flow of energy from the marine to terrestrial food webs and the absence of predation. Proceedings of the National Academy of Sciences of the United States of America, 92, 4382-4386.

POLIS, G. A. \& HURD, S. D. 1996. Linking marine and terrestrial food webs: allochthonous input from the ocean supports high secondary productivity on small islands and coastal land communities. American Naturalist, 147, 396-423.

POLIS, G. A., SÁNCHEZ-PIÑERO, F., STAPP, P. T., ANDERSON, W. B. \& ROSE, M. D. 2004. Trophic flows from water to land: marine input affects food webs of islands and coastal ecosystems worldwide. Food webs at the landscape level, 14, 209.

POLIS, G. A. \& STRONG, D. R. 1996. Food web complexity and community dynamics. American Naturalist, 813-846. 
POLIS, G. A. \& WINEMILLER, K. O. 1996. Food webs: integration of patterns and dynamics, Chapman and Hall.

POSSINGHAM, H. P., FRANKLIN, J., WILSON, K. \& REGAN, T. J. 2005. The roles of spatial heterogeneity and ecological processes in conservation planning. Ecosystem function in heterogeneous landscapes. Springer.

POST, D. M. 2002. Using stable isotopes to estimate trophic position: models, methods, and assumptions. Ecology, 83, 703-718.

POST, D. M., DOYLE, M. W., SABO, J. L. \& FINLAY, J. C. 2007a. The problem of boundaries in defining ecosystems: a potential landmine for uniting geomorphology and ecology. Geomorphology, 89, 111-126.

POST, D. M., LAYMAN, C. A., ARRINGTON, D. A., TAKIMOTO, G., QUATTROCHI, J. \& MONTANA, C. G. 2007b. Getting to the fat of the matter: models, methods and assumptions for dealing with lipids in stable isotope analyses. Oecologia, 152, 179-189.

PRESSEY, R. L., WATTS, M. E., BARRETT, T. W. \& RIDGES, M. J. 2009. The C-Plan conservation planning system: origins, applications, and possible futures.

RAJAKUMAR, A., ALAGARSAMY, R., KHARE, N., SARASWAT, R. \& SUBRAMANIAM, M. M. 2008. Studies on the nutrient distribution in the Southern Ocean waters along the $45 \mathrm{oE}$ transect. Indian Journal of Marine Sciences, 37, 424-429.

REDFIELD, A. C. 1958. The biological control of chemical factors in the environment. American Scientist, 46.

REMOUNDOU, K., KOUNDOURI, P., KONTOGIANNI, A., NUNES, P. A. L. D. \& SKOURTOS, M. 2009. Valuation of natural marine ecosystems: an economic perspective. Environmental Science and Policy, 12, 1040-1051.

ROBERTS, R. D., KAWAMURA, T. \& NICHOLSON, C. M. 1999. Growth and survival of postlarval abalone (Haliotis iris) in relation to development and diatom diet. Journal of Shellfish Research, 18, 243-250.

ROBERTSON, C. J. R. \& VAN TETS, G. F. 1982. The status of birds at the Bounty Islands. Notornis, 29, 311-336.

ROONEY, N., MCCANN, K., GELLNER, G. \& MOORE, J. C. 2006. Structural asymmetry and the stability of diverse food webs. Nature, 442, 265-269.

ROUNICK, J. S. \& WINTERBOURN, M. J. 1986. Stable carbon isotopes and carbon flow in ecosystems. BioScience, 36, 171-177.

RUCKELSHAUS, M., KLINGER, T., KNOWLTON, N. \& DEMASTER, D. P. 2008. Marine Ecosystem-based Management in Practice: Scientific and Governance Challenges. BioScience, 58, 53.

RYTHER, J. H. \& DUNSTAN, W. M. 1971. Nitrogen, phosphorus, and eutrophication in the coastal marine environment. Science (New York, NY), 171, 1008.

SAMHOURI, J. F. \& LEVIN, P. S. 2012. Linking land- and sea-based activities to risk in coastal ecosystems. Biological Conservation, 145, 118.

SÁNCHEZ-PIÑERO, F. \& POLIS, G. A. 2000. Bottom-up dynamics of allochthonous input: direct and indirect effects of seabirds on islands. Ecology, 81, 3117-3132.

SCHMITZ, O. J., HAMBÄCK, P. A. \& BECKERMAN, A. P. 2000. Trophic cascades in terrestrial systems: a review of the effects of carnivore removals on plants. The American Naturalist, 155, 141-153. 
SHEARS, N. T. \& BABCOCK, R. C. 2002. Marine reserves demonstrate topdown control of community structure on temperate reefs. Oecologia, 132, 131-142.

SOREIDE, J. E., TAMELANDER, T., HOP, H., HOBSON, K. A. \& JOHANSEN, I. 2006. Sample preparation effects on stable C and N isotope values: a comparison of methods in Arctic marine food web studies. Marine Ecology Progress Series, 328, 17-28.

SPEAR, L. B., AINLEY, D. G. \& WEBB, S. W. 2003. Distribution, abundance and behaviour of Buller's, Chatham Island and Salvin's Albatrosses off Chile and Peru. Ibis, 145, 253-269.

SPSS 2006. SPSS for Windows15.0.0. Chicago, IL: SPSS Inc.

STOMS, D. M., DAVIS, F. W., ANDELMAN, S. J., CARR, M. H., GAINES, S. D., HALPERN, B. S., HOENICKE, R., LEIBOWITZ, S. G., LEYDECKER, A., MADIN, E. M. P., TALLIS, H. \& WARNER, R. R. 2005. Integrated coastal reserve planning: making the land-sea connection. Frontiers in Ecology, 3, 429-436.

SUBANTARCTIC MARINE PROTECTION PLANNING FORUM 2009. Implementation of the marine protected areas policy in the territorial sea of the subantarctic biogeographic region of New Zealand: Consultation document.

SUMMERHAYES, V. \& ELTON, C. S. 1923. Contributions to the ecology of Spitsbergen and Bear Island, University Press.

SYDEMAN, W. J., HOBSON, K. A., PYLE, P. \& MCLAREN, E. B. 1997. Trophic relationships among seabirds in central California: combined stable isotope and conventional dietary approach. Condor, 327-336.

TATUR, A. \& MYRCHA, A. 1983. Changes in chemical composition of waters running off from the penguin rookeries in the Admiralty Bay region(King George Island, South Shetland Islands, Antarctica). POL. POLAR RES., 4, 113-126.

TAYLOR, R. 2006. Straight through from London: The Antipodes and Bounty Islands, New Zealand, Christchurch, Heritage Expeditions New Zealand Ltd.

TAYLOR, R. H. 1996. Distribution, abundance and pup production of the New Zealand fur seal (Arctocephalus forsteri Lesson) at the Bounty Islands. Wellington, New Zealand: Department of Conservation.

TAYLOR, R. H. \& TAYLOR, G. A. 1989. Re-assessment of the status of southern elephant seals (Mirounga leonina) in New Zealand. New Zealand Journal of Marine and Freshwater Research, 23, 201-213.

THOMAS, T. E. \& HARRISON, P. J. 1987. Rapid ammonium uptake and nitrogen interactions in five intertidal seaweeds grown under field conditions. Journal of Experimental Marine Biology and Ecology, 107, 18.

THOMPSON, R. M., HEMBERG, M., STARZOMSKI, B. M. \& SHURIN, J. B. 2007. Trophic levels and trophic tangles: the prevalence of omnivory in real food webs. Ecology, 88, 612-617.

THOMPSON, R. M., POULIN, R., MOURITSEN, K. N. \& THIELTGES, D. W. 2013. Resource tracking in marine parasites: going with the flow? Oikos.

TOONEN, R. J., ANDREWS, K. R., BAUMS, I. B., BIRD, C. E., CONCEPCION, G. T., DALY-ENGEL, T. S., EBLE, J. A., FAUCCI, A., GAITHER, M. R. \& IACCHEI, M. 2011. Defining boundaries for 
ecosystem-based management: a multispecies case study of marine connectivity across the Hawaiian Archipelago. Journal of Marine Biology, 2011.

TRULL, T. \& ARMAND, L. 2001. Insights into Southern Ocean carbon export from the D13C of particles and dissolved inorganic carbon during the SOIREE iron release experiment. Deep Sea Research Part II: Topical Studies in Oceanography, 48, 2655-2680.

VANDER ZANDEN, M. J. \& RASMUSSEN, J. B. 2001. Variation in $\delta 15 \mathrm{~N}$ and $\delta 13 \mathrm{C}$ trophic fractionation: implications for aquatic food web studies. Limnology and oceanography, 2061-2066.

VASIL, C. A., POLITO, M. J., PATTERSON, W. P. \& EMSLIE, S. D. 2012. Wanted: dead or alive? Isotopic analysis $(\delta 13 \mathrm{C}$ and $\delta 15 \mathrm{~N})$ of Pygoscelis penguin chick tissues supports opportunistic sampling. Rapid Communications in Mass Spectrometry, 26, 487-493.

VITOUSEK, P. M. 2002. Oceanic islands as model systems for ecological studies. Journal of Biogeography, 29, 573-582.

VITOUSEK, P. M. \& HOWARTH, R. W. 1991. Nitrogen limitation on land and in the sea: how can it occur? Biogeochemistry, 13, 87-115.

WAINRIGHT, S. C., FOGARTY, M. J., GREENFIELD, R. C. \& FRY, B. 1993. Long-term changes in the Georges Bank food web: Trends in stable isotopic compositions of fish scales. Marine Biology, 115, 481-493.

WAINRIGHT, S. C., HANEY, J. C., KERR, C., GOLOVKIN, A. N. \& FLINT, M. V. 1998. Utilization of nitrogen derived from seabird guano by terrestrial and marine plants at St. Paul, Pribilof Islands, Bering Sea, Alaska. Marine Biology, 131, 63-71.

WARDLE, D. A. 2002. Islands as model systems for understanding how species affect ecosystem properties. Journal of Biogeography, 29, 583-591.

WARHAM, J. \& BELL, B. D. 1979. The birds of Antipodes Island, New Zealand. Notornis, 26, 121-169.

WATTS, M. E., BALL, I. R., STEWART, R. S., KLEIN, C. J., WILSON, K., STEINBACK, C., LOURIVAL, R., KIRCHER, L. \& POSSINGHAM, H. P. 2009. Marxan with zones: software for optimal conservation based land- and sea-use zoning. Environmental Modelling and Software, 1-9.

WHITTAKER, R. J. \& FERNÁNDEZ-PALACIOS, J. M. 2007. Island biogeography: ecology, evolution, and conservation, Oxford University Press.

WIENECKE, B. \& ROBERTSON, G. 2002. Foraging areas of king penguins from Macquarie Island in relation to a marine protected area. Environmental Management, 29, 662-672.

WILLIS, T. in press. Scientific and biodiversty values of marine reserves: a review. Wellington, New Zealand.

WILLIS, T., SWEETING, C., BURY, S., HANDLEY, S., BROWN, J., FREEMAN, D., CAIRNEY, D. \& PAGE, M. 2013. Matching and mismatching stable isotope $(\delta 15 \mathrm{~N}$ and $\delta 13 \mathrm{C})$ ratios among fish species in fin and muscle tissue: a critical review. Marine Biology, 160, 1633-1644.

WISE, D. H. 2006. Cannibalism, food limitation, intraspecific competition, and the regulation of spider populations. Annu. Rev. Entomol., 51, 441-465.

WITMAN, J. D., ELLIS, J. C. \& ANDERSON, W. B. 2004. The influence of physical processes, organisms, and permeability on cross-ecosystem 
fluxes. Food webs at the landscape level. University of Chicago Press, Chicago, Illinois, USA, 335-358.

WOOTTON, J. T. 1991. Direct and indirect effects of nutrients on intertidal community structure: variable consequences of seabird guano. Journal of Experimental Marine Biology and Ecology, 151, 139-153.

WOOTTON, J. T. 1995. Effects of birds on sea urchins and algae: A lower intertidal trophic cascade. Ecoscience, 2, 321-328.

YOUNG, O. R., OSHERENKO, G., EKSTROM, J., CROWDER, L. B., OGDEN, J., WILSON, J. A., DAY, J. C., DOUVERE, F., EHLER, C. N. \& MCLEOD, K. L. 2007. Solving the crisis in ocean governance: placebased management of marine ecosystems. Environment: science and policy for sustainable development, 49, 20-32.

ZANDEN, M. J. V. \& RASMUSSEN, J. B. 1999. Primary consumer $\delta 13 \mathrm{C}$ and $\delta 15 \mathrm{~N}$ and the trophic position of aquatic consumers. Ecology, 80, 13951404.

ZWOLICKI, A., ZMUDCZYNSKA-SKARBEK, K. M. G., ILISZKO, L. \& STEMPNIEWICZ, L. 2012. Guano deposition and nutrient enrichment in the vicinity of planktivorous and piscivorous seabird colonies in Spitsbergen. Polar Biology, 36, 363. 
APPENDIX 1 Diet of the holothurian Squamocnus brevidentis, at Antipodes Island, using Isosource.
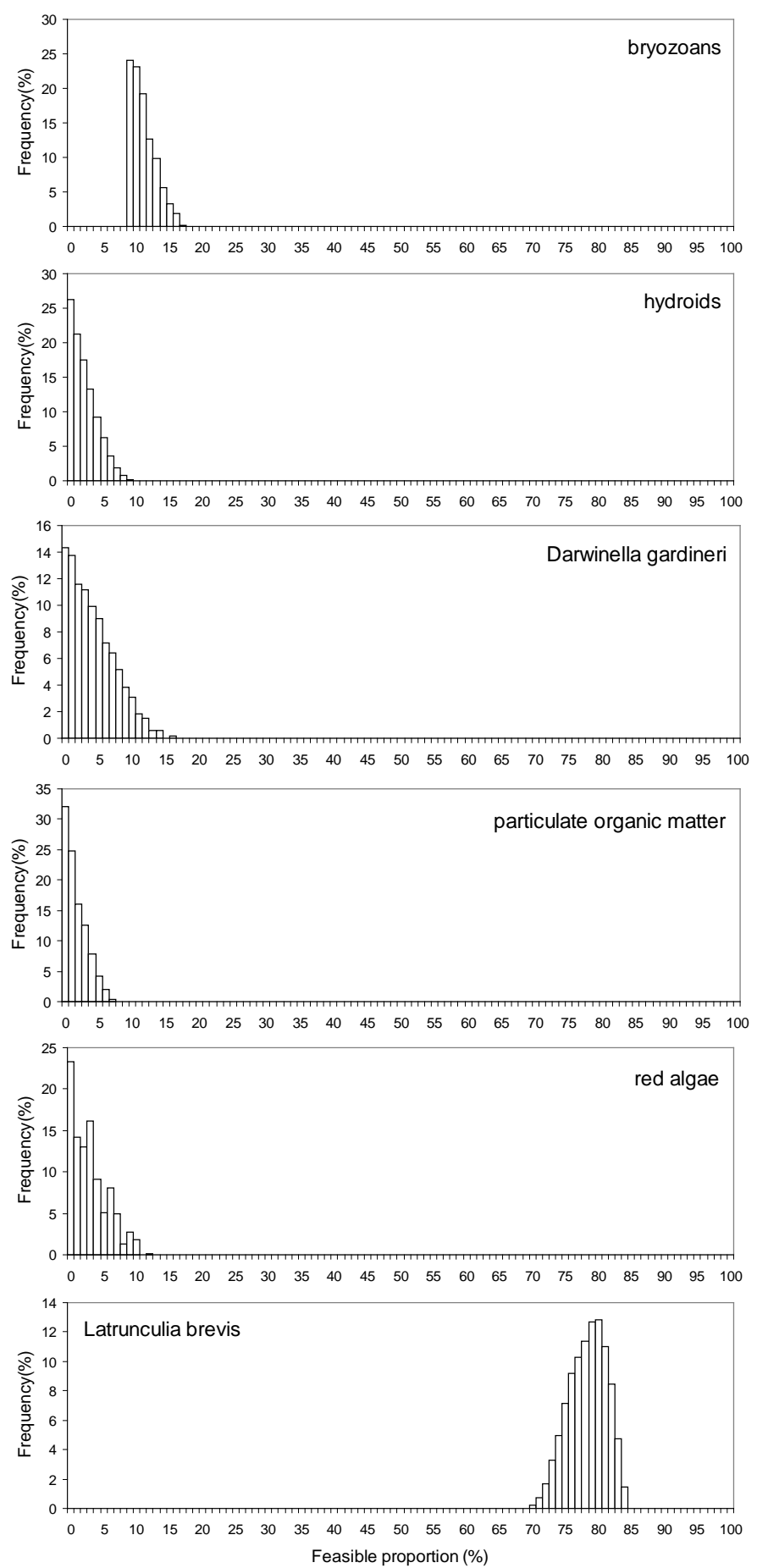
APPENDIX 2 Diet of shrimp at the Bounty Islands, using Isosource.
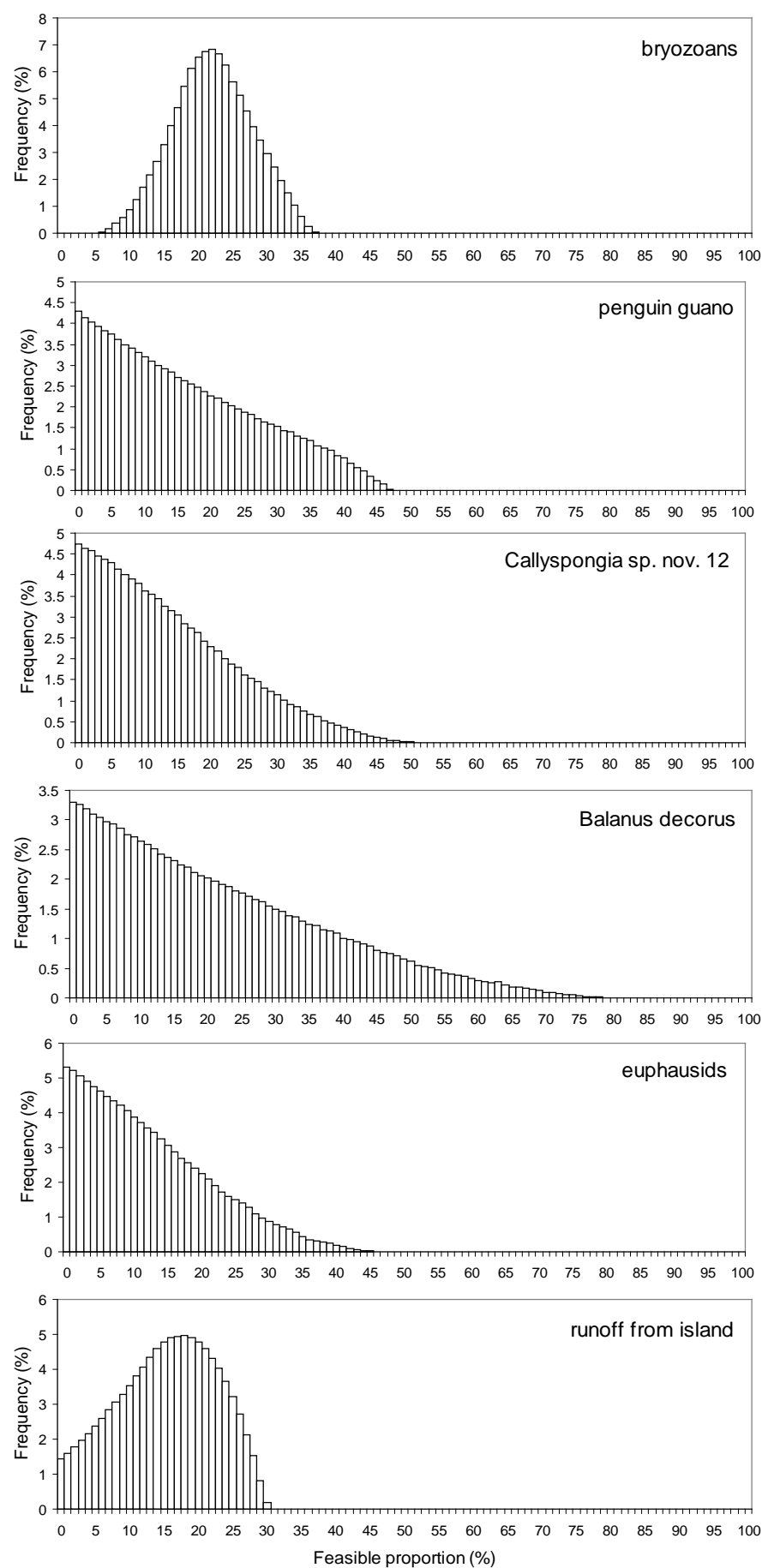
APPENDIX 3 Diet of the starfish Paranepanthia, At Antipodes Island, using Isosource.
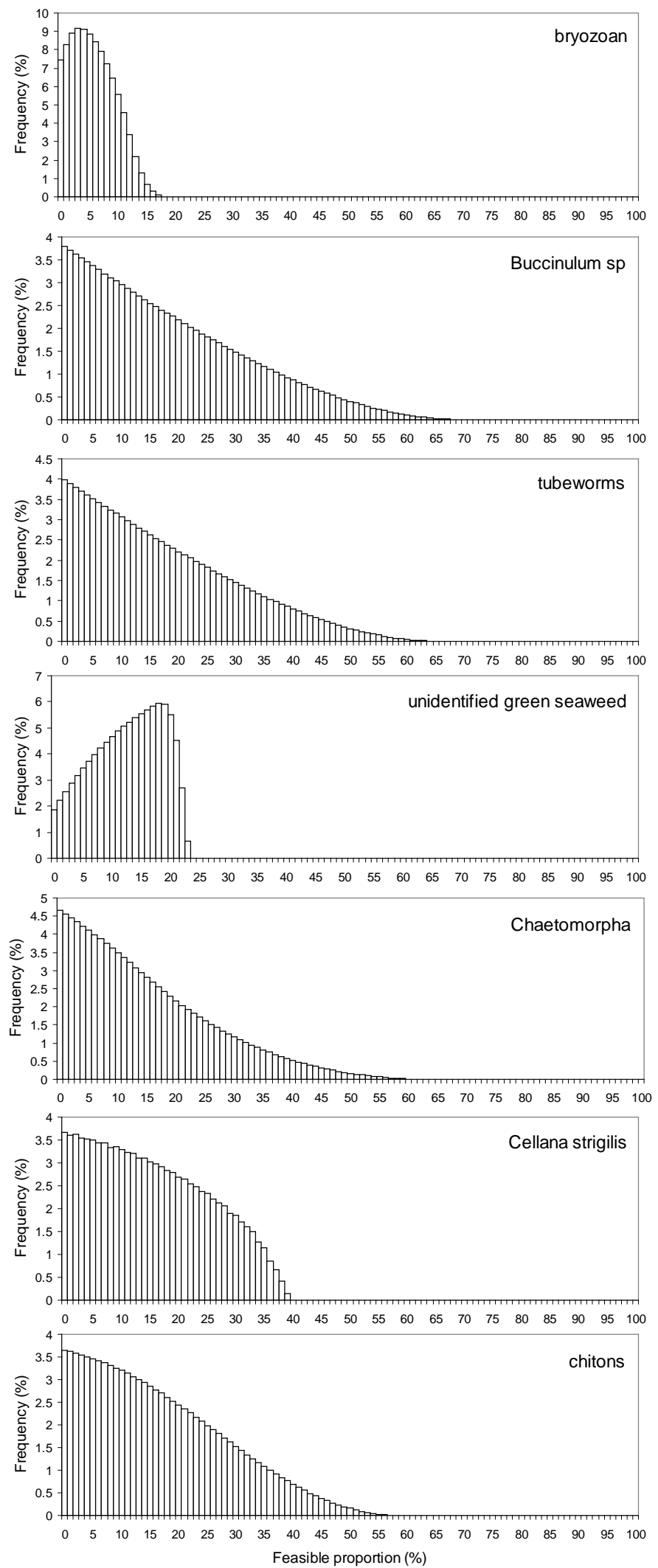
APPENDIX 4 Diet of chiton Onithochiton neglectus, at the Bounty Islands, using Isosource.
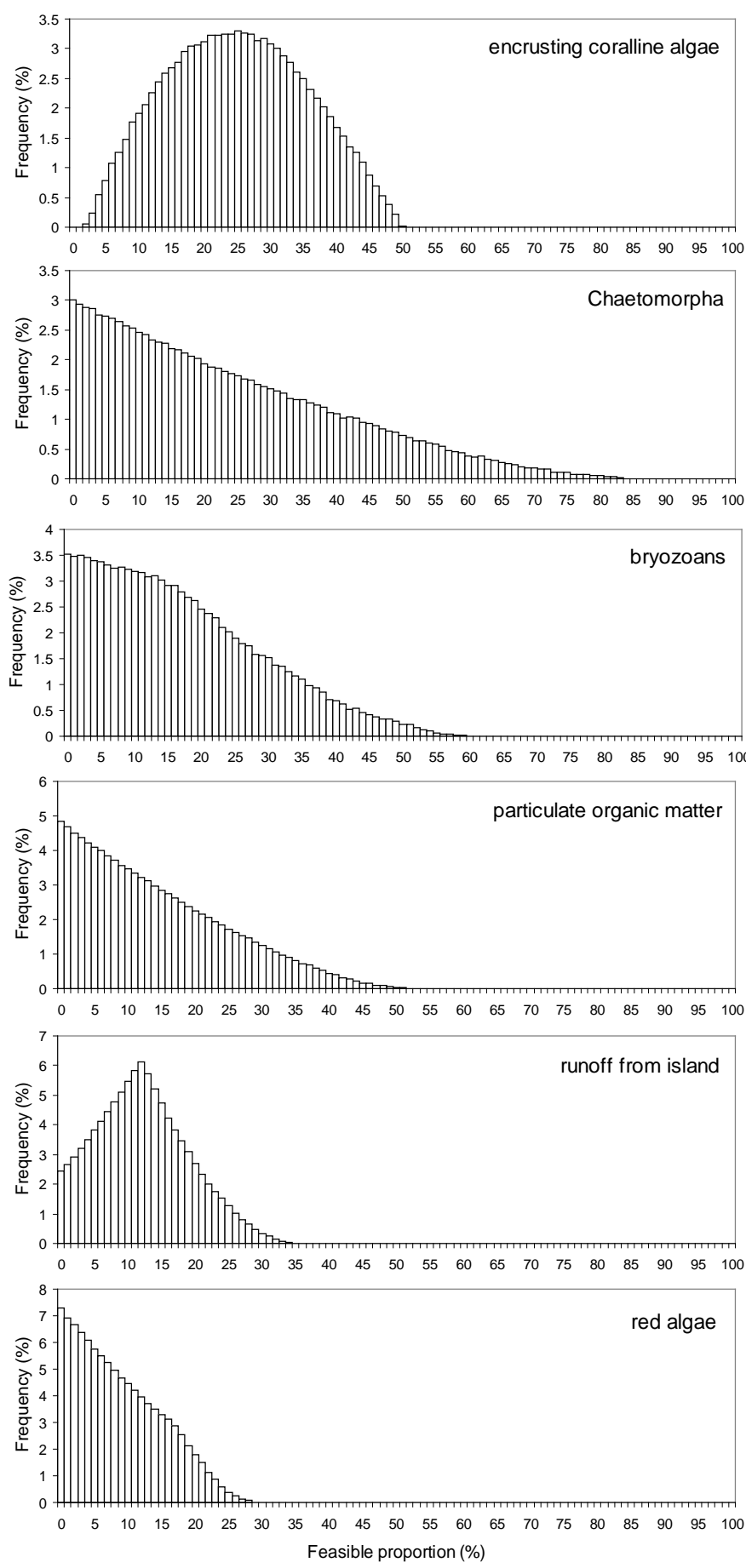
APPENDIX 5 Diet of Henricia, at Antipodes Island, using Isosource.
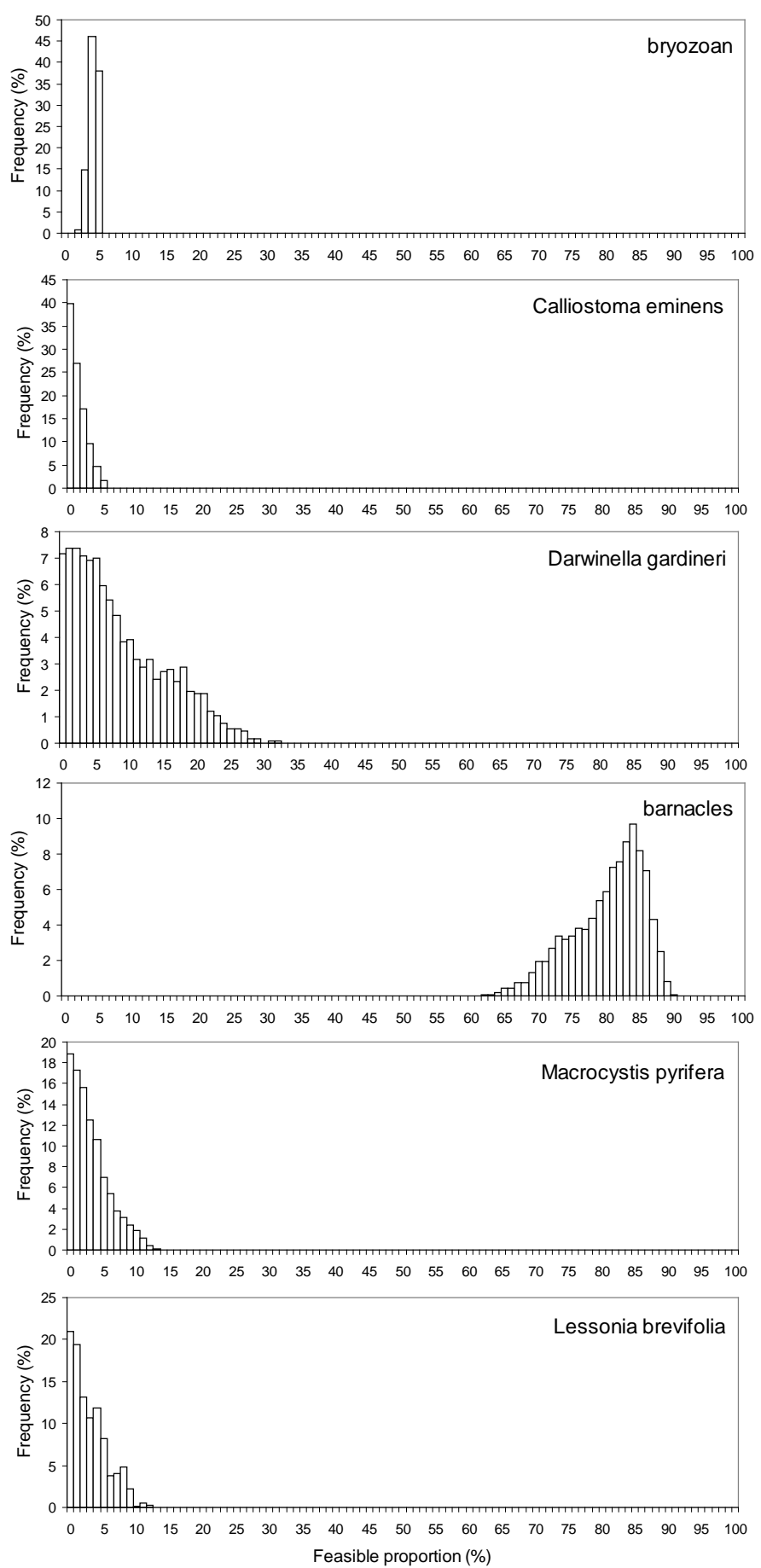
APPENDIX 6 Diet of starfish Henricia, at the Bounty Islands, using Isosource.
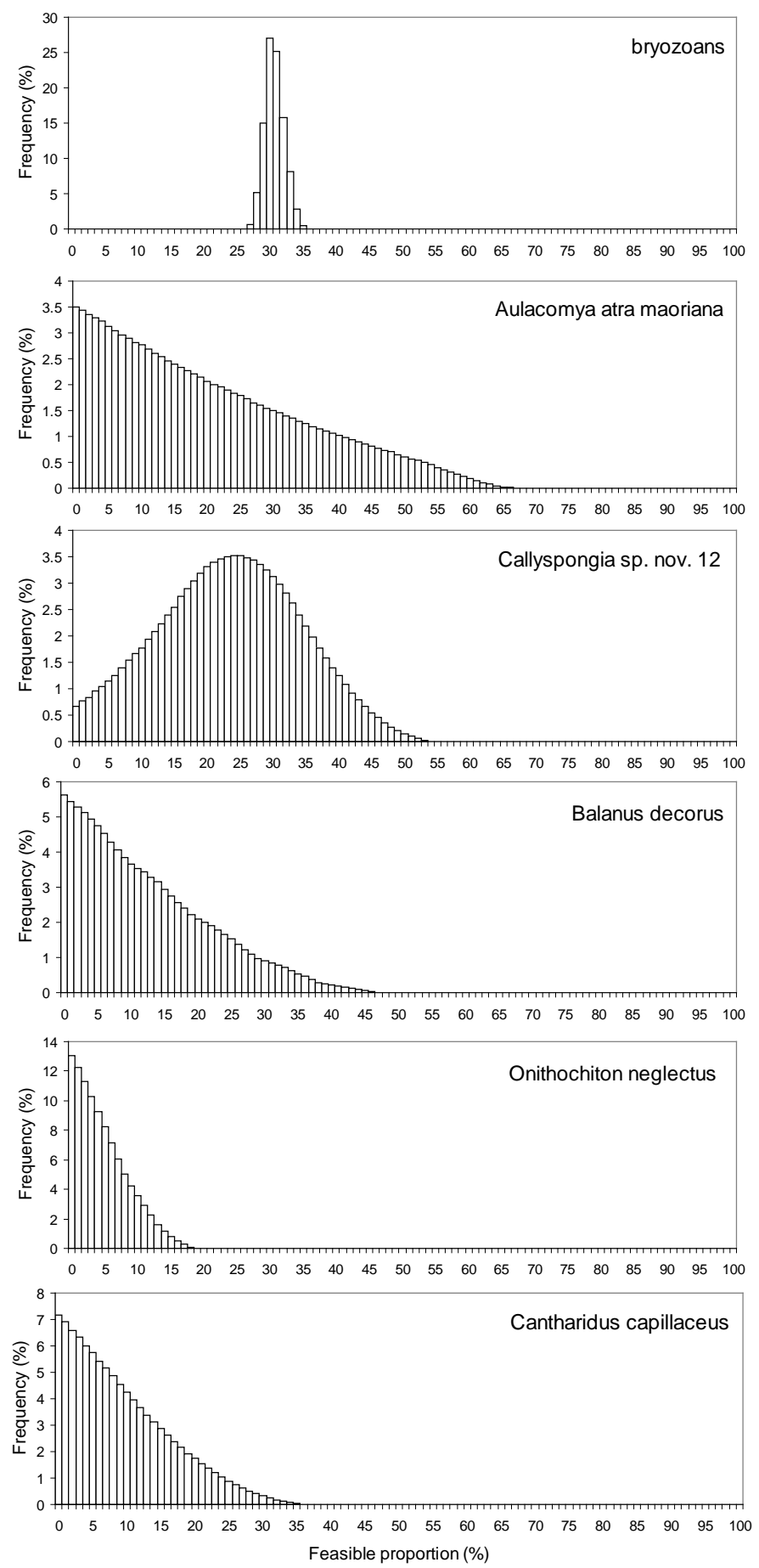
APPENDIX 7 Diet of the paua Haliotis virginea huttoni, at Antipodes Island, using Isosource.
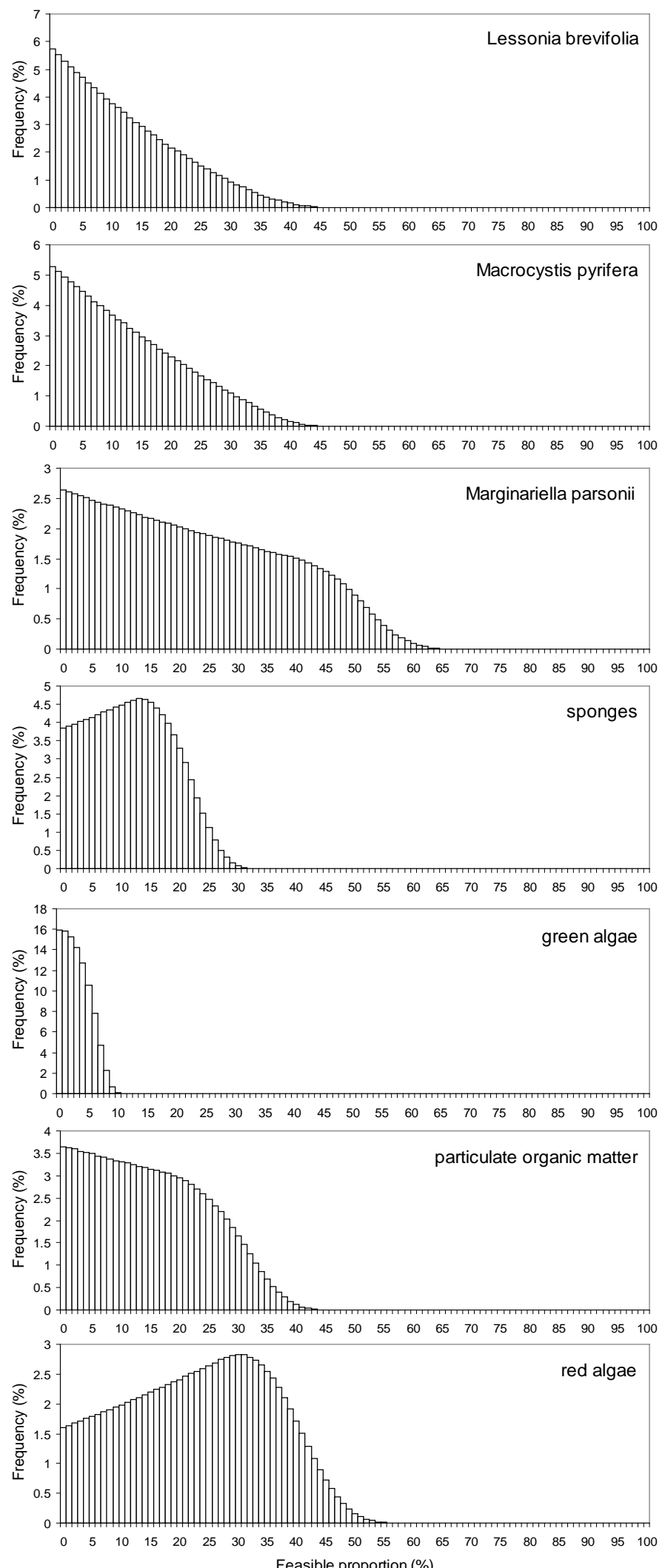
APPENDIX 8 Diet of paua Haliotis virginea huttoni, at the Bounty Islands, using Isosource.
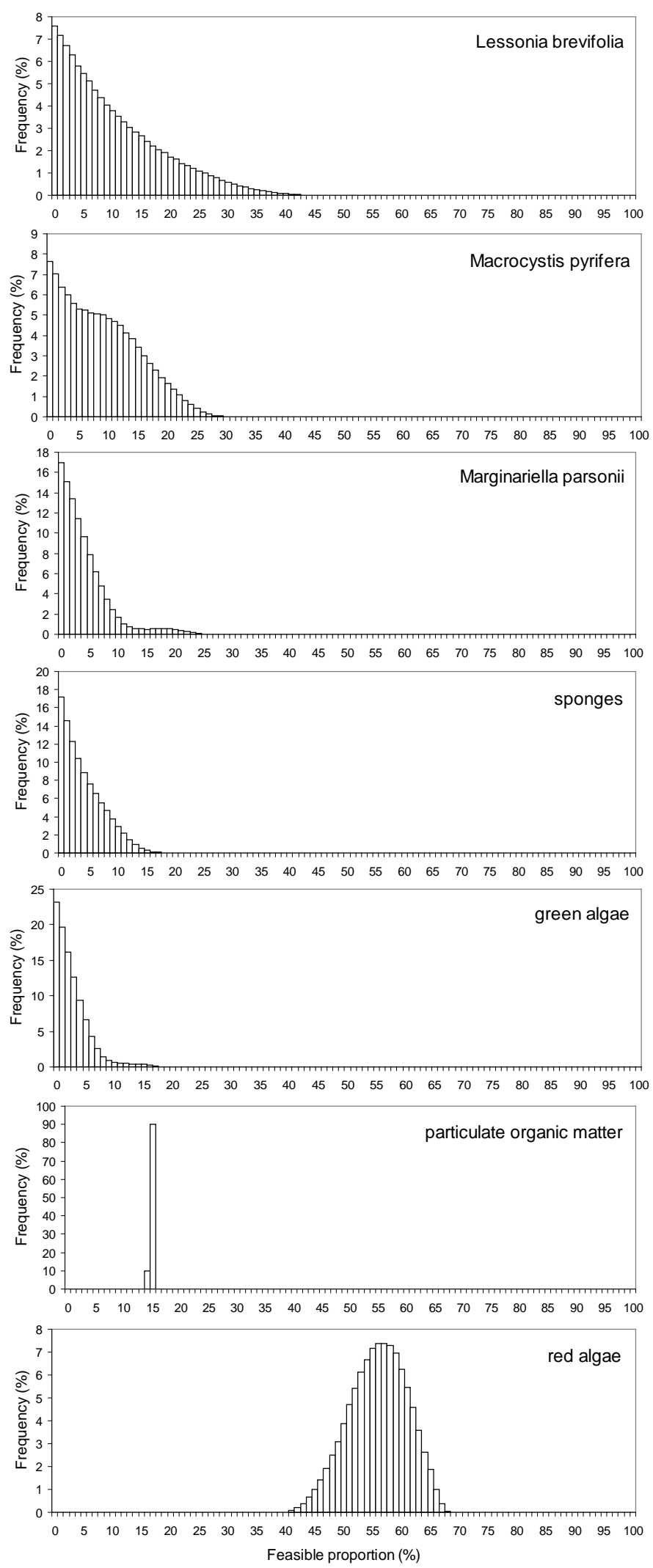
APPENDIX 9 Diet of Calliostoma at Antipodes Island, using Isosource.
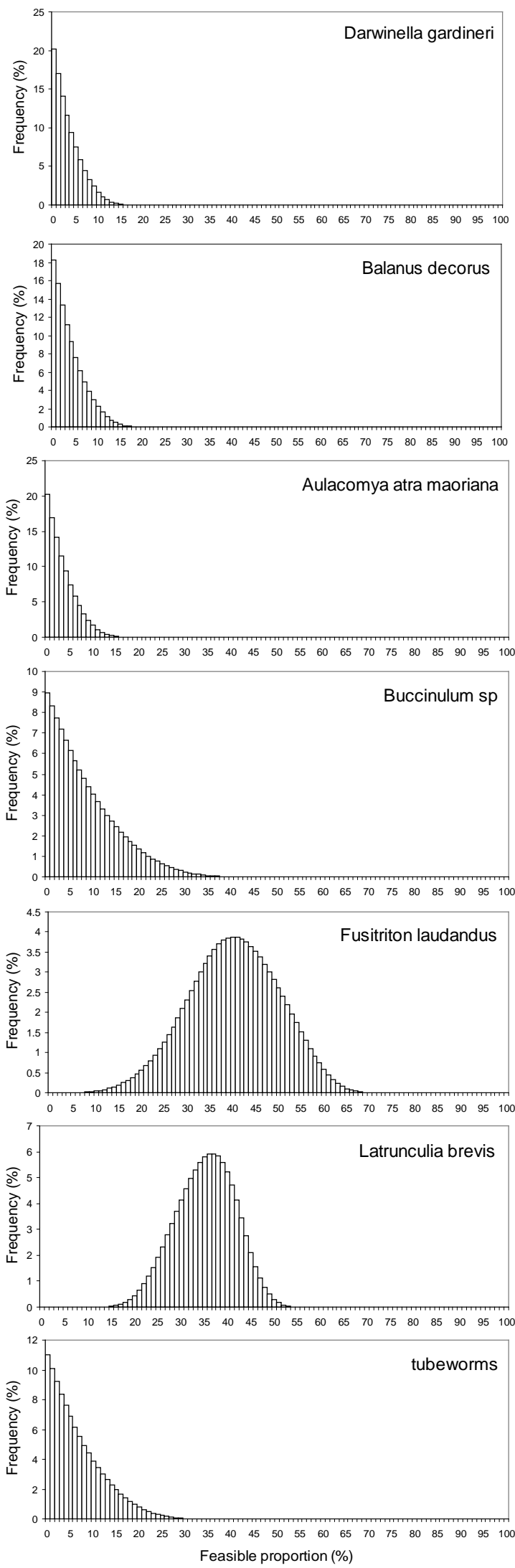
APPENDIX 10 Diet of whelk Haustrum, at the Bounty Islands, using Isosource.
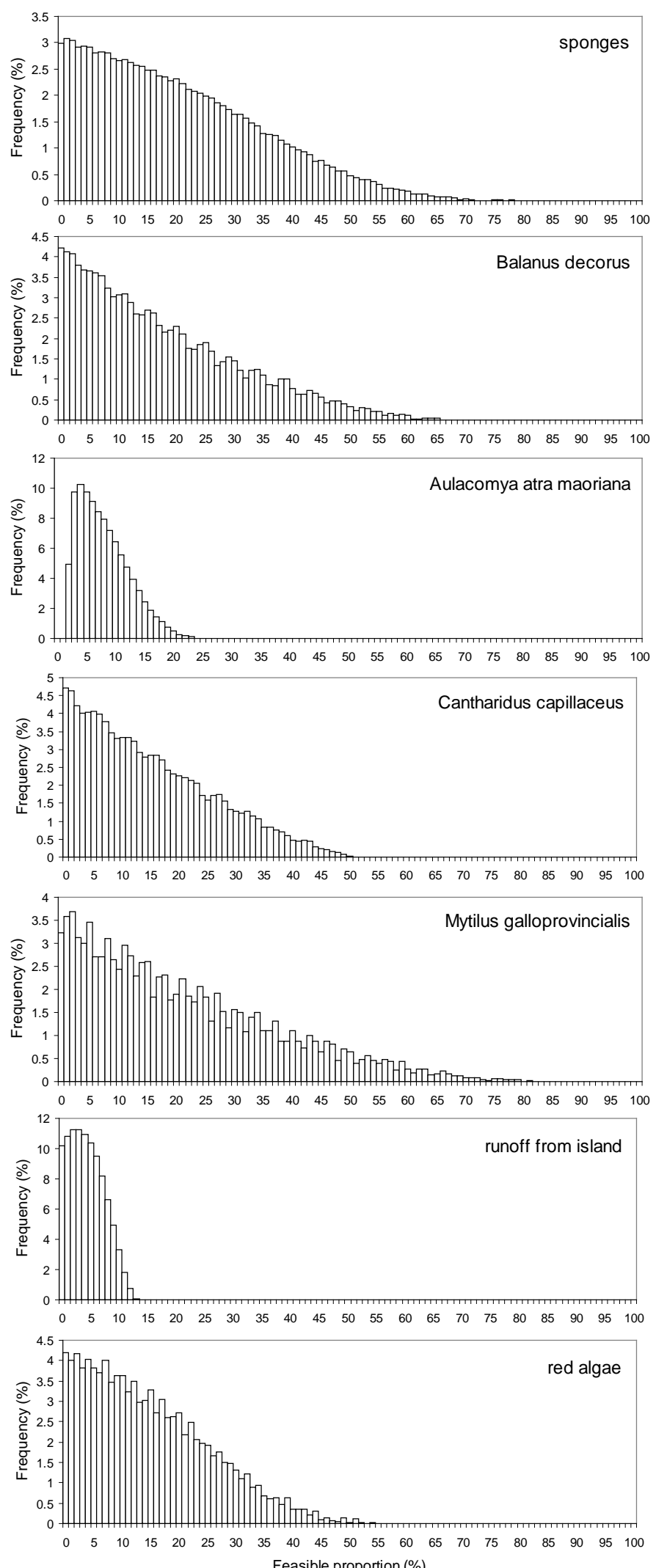
APPENDIX 11 Diet of Antipodes parakeet, Cyanoramphus unicolor using Isosource.
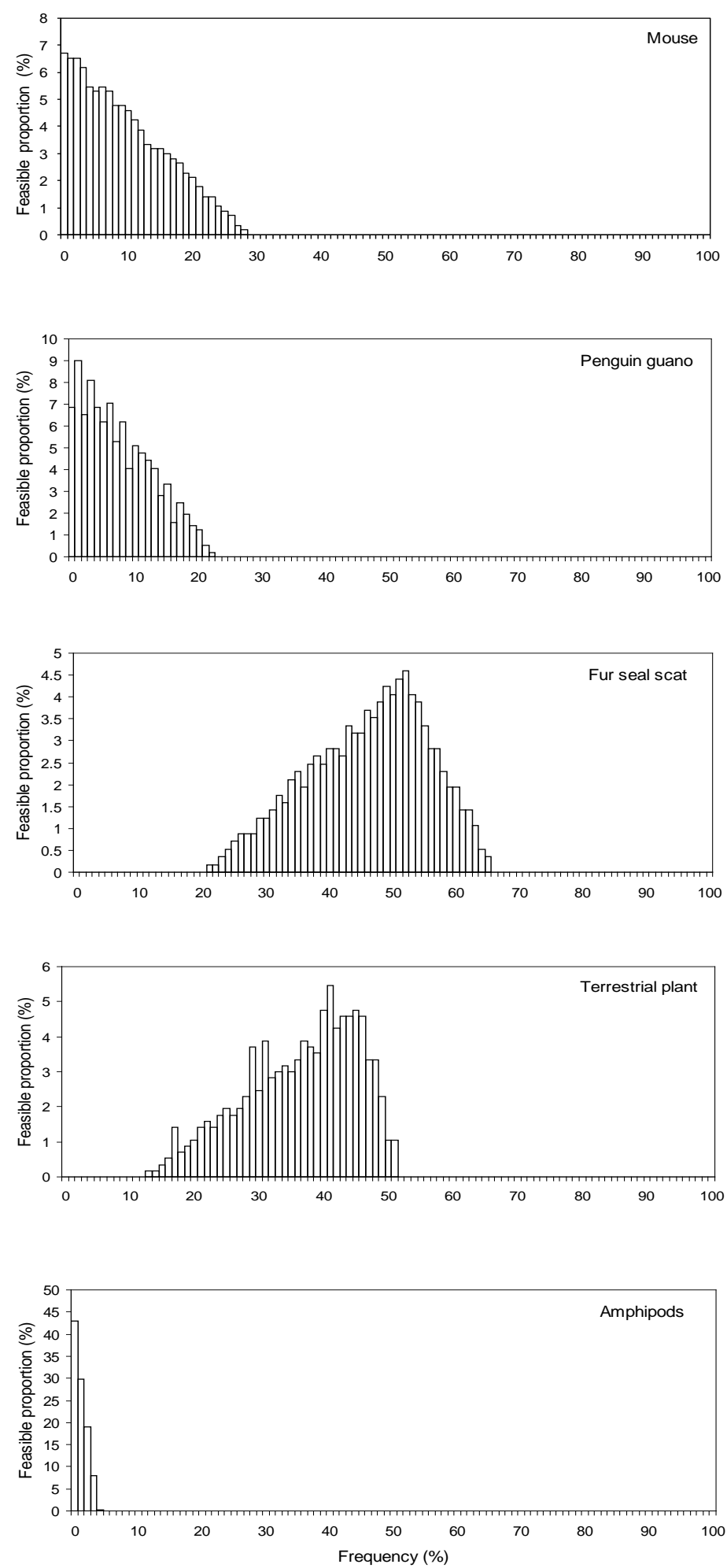
APPENDIX 12 Diet of red-crowned parakeet, Cyanoramphus novaezelandiae hochstetteri at Antipodes Island, using Isosource.
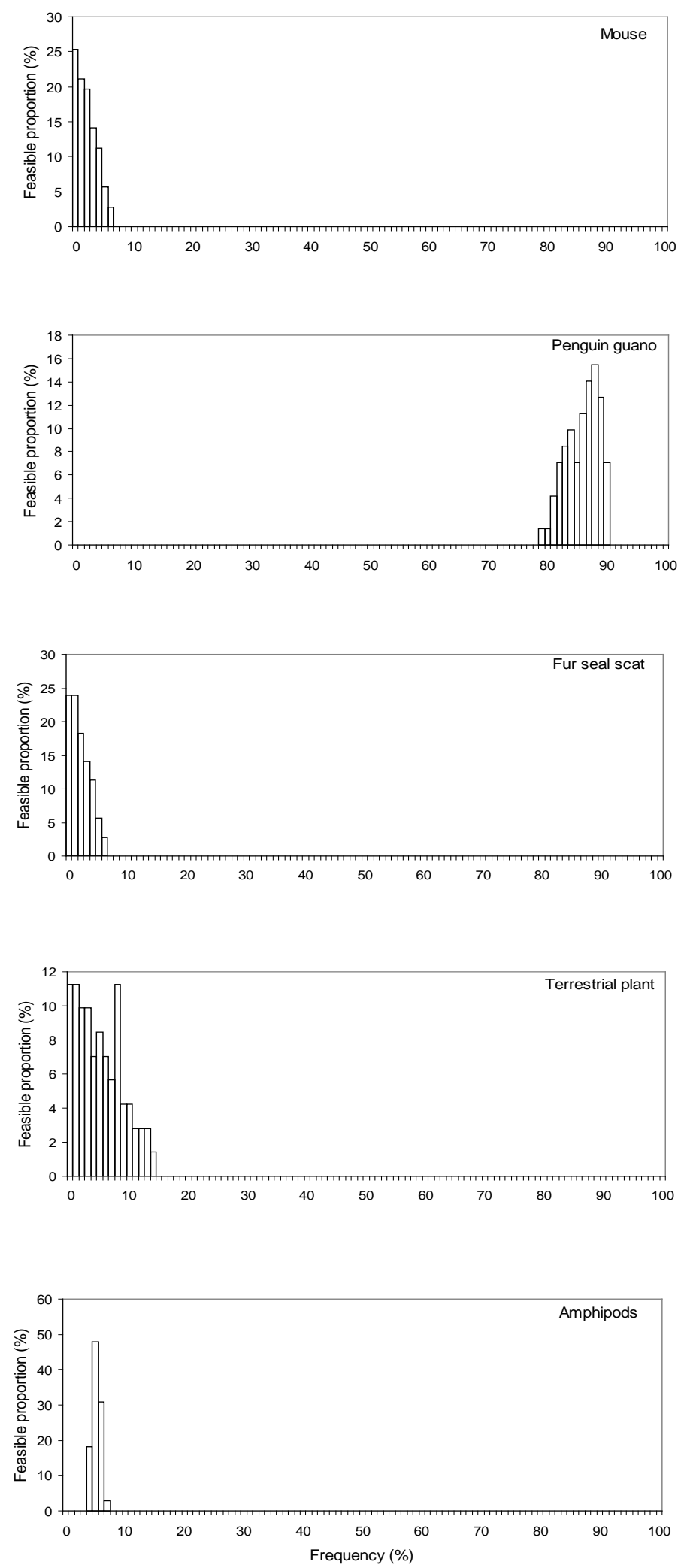
APPENDIX 13 Diet of Antipodes Island Snipe using Isosource.
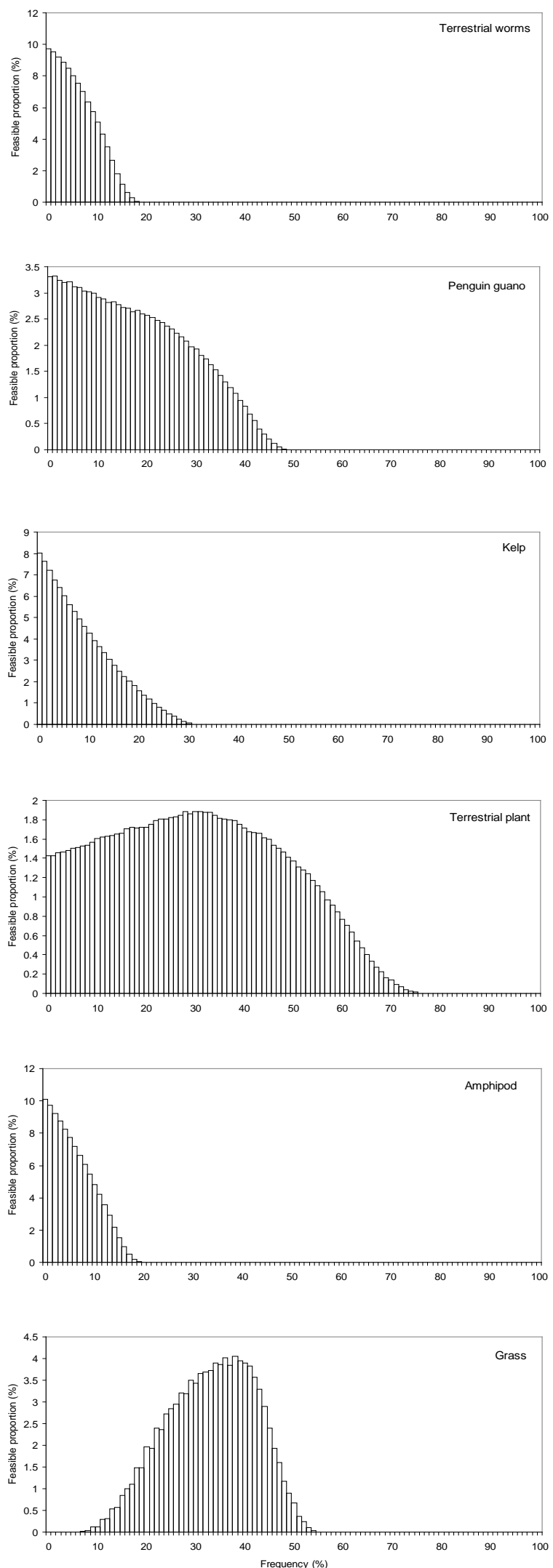
APPENDIX 14 Diet of mouse Mus musculus at Antipodes Island, using Isosource.
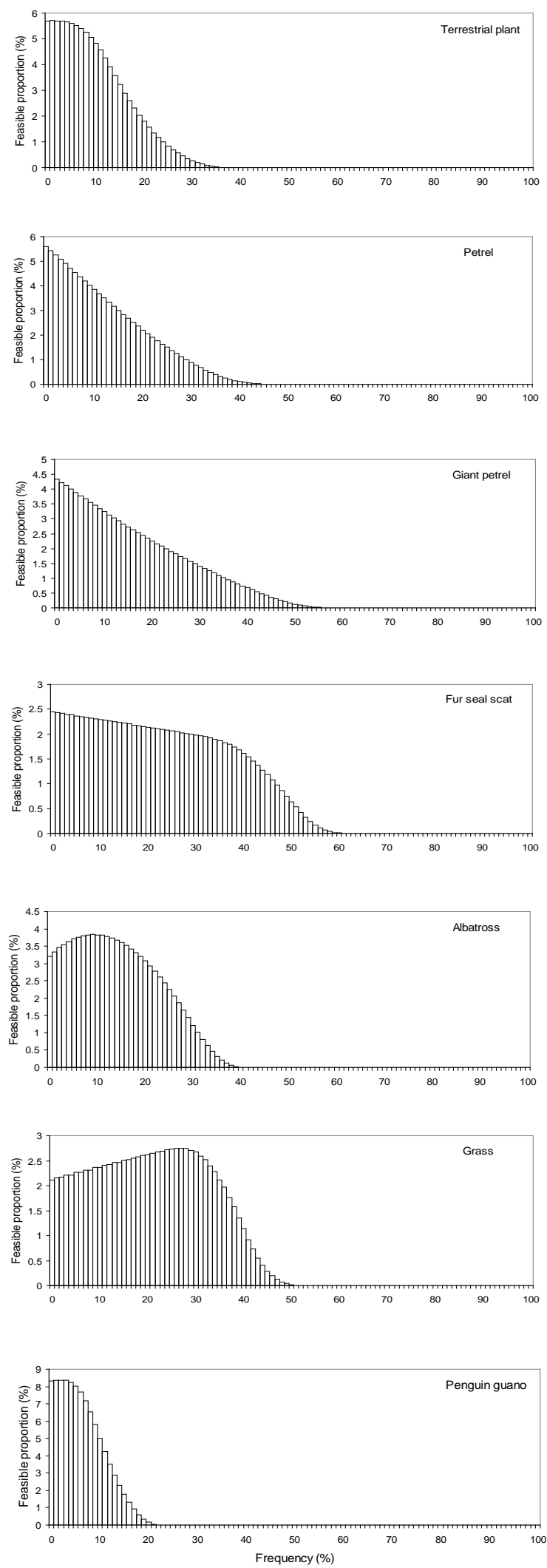
APPENDIX 15 Diet of spider Pacificana at the Bounty Islands, using Isosource.
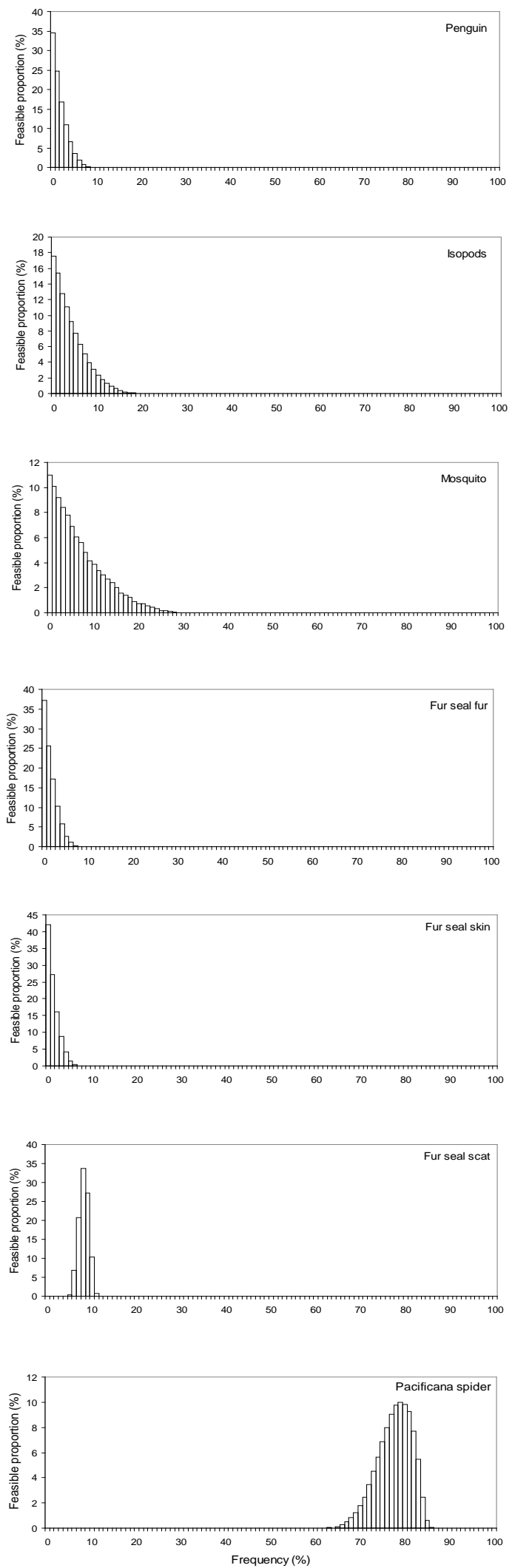